\title{
Souffle/Spastizin regulates secretory granule maturation by sorting lysosomal cargo from immature secretory granule during zebrafish oogenesis.
}

\author{
$\mathrm{PhD}$ Thesis \\ in partial fulfillment of the requirements \\ for the degree "Doctor rerum naturalium (Dr.rer.nat)" \\ in the GGNB Program: "Genes and Development" \\ at the Georg August University Göttingen \\ Faculty of Biology
}

Submitted by

Palsamy Kanagaraj

Born in Pulvoikulam, Tamil Nadu, India

September 2014 


\section{Thesis Committee Members}

\section{Dr.Roland Dosch}

Dept. of Developmental Biochemistry,

Justus-Von-Liebig-Weg 11, GZMB, 37077, Goettingen, Germany.

\section{Prof. Dr. Andreas Wodarz}

Dept.of Anatomy and Stem cell biology,

Justus-Von-Liebig-Weg 11, GZMB, 37077, Goettingen, Germany.

Current: Institute I for Anatomy, Gebäude 35, Joseph-Stelzmann-Str. 9, 50931

Köln, Germany.

3. Prof. Dr. Mikael Simons

Dept. of Neurology, MPI for Experimental Medicine,

Hermann-Rein Str. 3, 37075 Göttingen, Germany.

\section{Extended Thesis Committee Members}

1. Prof. Dr. Ernst Wimmer

Dept. of Developmental Biology,

Justus-Von-Liebig-Weg 11, GZMB, 37077, Goettingen, Germany.

2. Prof. Dr. Gregor Bucher

Dept. of Developmental Biology,

Justus-Von-Liebig-Weg 11, GZMB, 37077, Goettingen, Germany.

3. Prof. Dr. Steven Johnson

Clinic for General, Visceral and Pediatric Surgery

UBFT 3-C2-514, Robert-Koch-Straße 40, 37075 Göttingen, Germany.

Date of the oral examination: 


\begin{abstract}
Affidavit
Herewith I declare that I prepared the $\mathrm{PhD}$ thesis "Souffle/Spastizin regulates secretory granule maturation by sorting lysosomal cargo from immature secretory granule during zebrafish oogenesis" on my own and with no other sources and aids than quoted.
\end{abstract}

30.09.2014

Submission date

Palsamy Kanagaraj 


\section{List of Publications}

1. Palsamy Kanagaraj, Gautier-Stein A, Dietmar Riedel, Christoph Schomburg, Joan Cerda`, Nadine vollock, Roland Dosch. (2014) Souffle/Spastizin Controls Secretory Vesicle Maturation during Zebrafish Oogenesis. PLoS Genet. 10(6): e1004449. doi:10.1371/journal.pgen.1004449

2. Palsamy Kanagaraj, Dietmar Riedel, Joan Cerda', Nore crascanta, Susanne Schlick, Roland Dosch. Souffle regulates sorting of lysosomal cargo from immature secretory granule in VPS41 transport pathway. Manuscript in preparation. 


\section{Table of Contents}

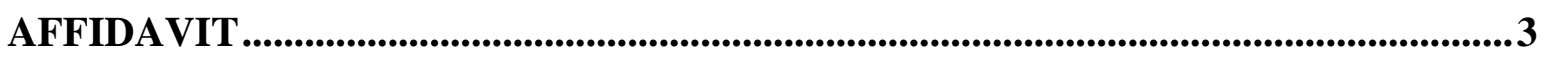

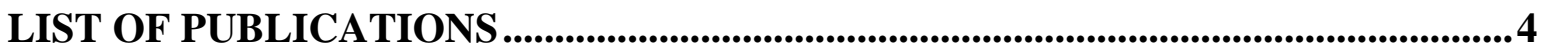

TABLE OF CONTENTS ........................................................................................................5

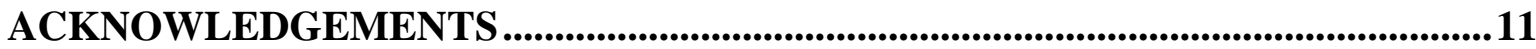

ABSTRACT .................................................................................................................................12

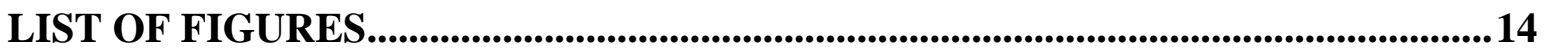

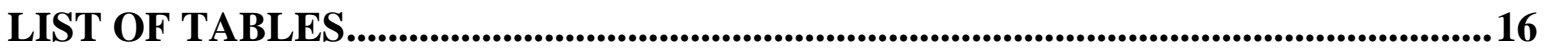

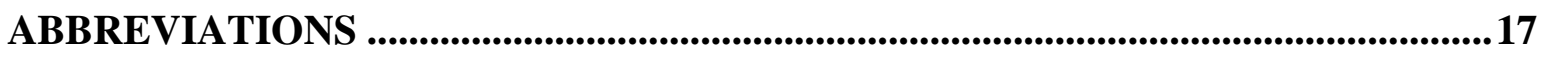

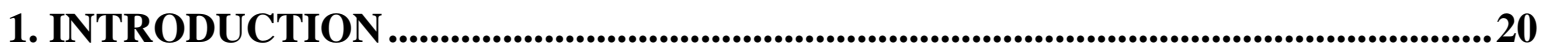

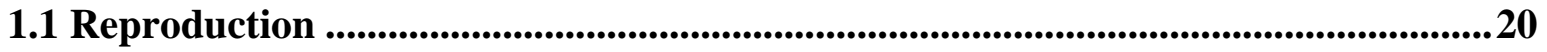

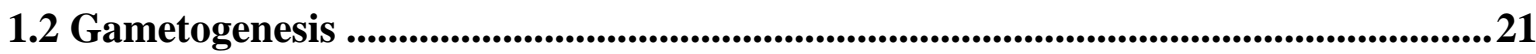

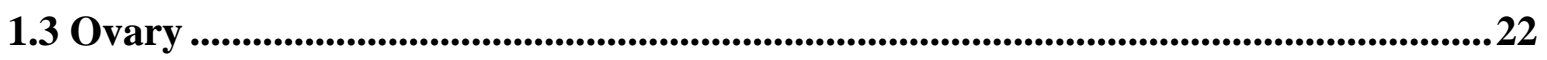

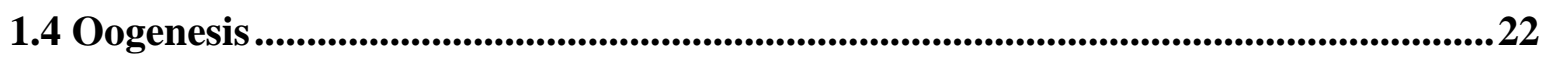

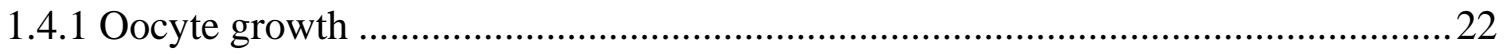

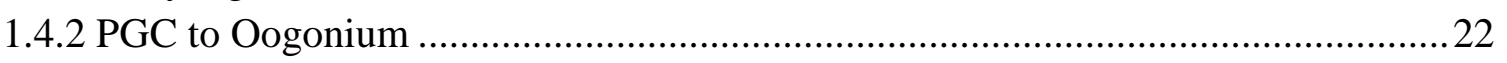

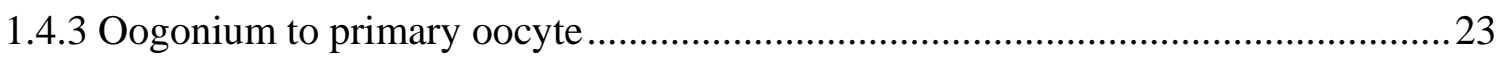

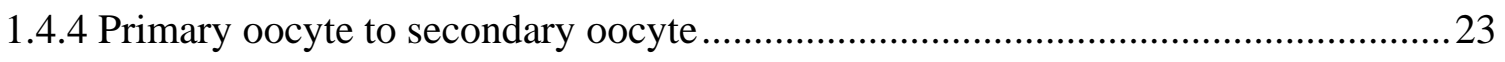

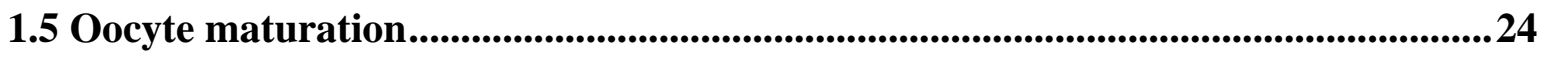

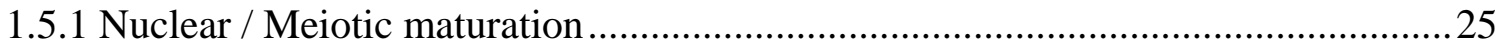

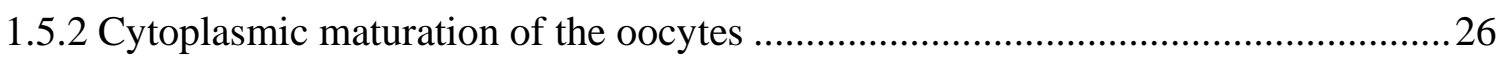

1.6 Hormonal induction of oocyte maturation....................................................................29

1.7 Essential developmental events during oogenesis........................................................30

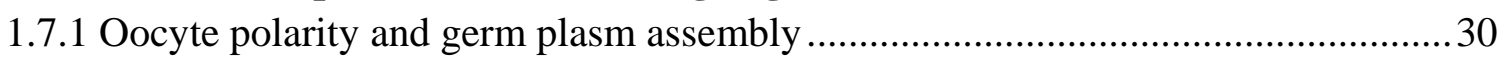

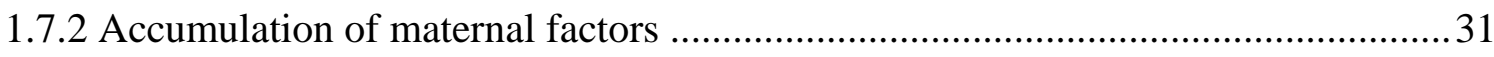

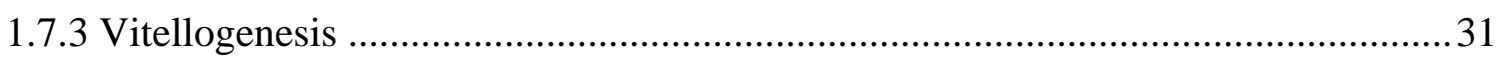

1.8 Vesicle trafficking during oogenesis ....................................................................32 


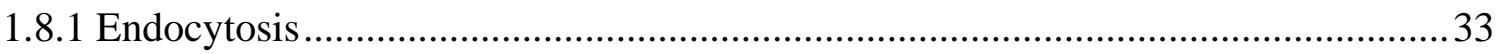

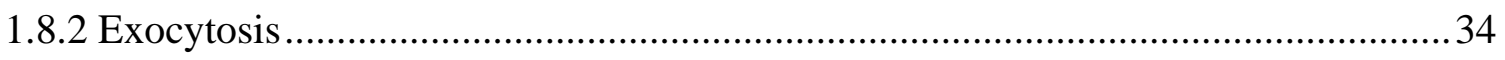

1.9 Investigating the event of yolk endocytosis in invertebrates ........................................36

1.10 Discovering novel regulators of oogenesis in vertebrate..............................................37

1.11 Zebrafish-vertebrate model...................................................................................................37

1.12 Zebrafish oogenesis .........................................................................................................................38

1.13 Genetic screen in zebrafish oocyte .....................................................................................40

1.14 Opaque egg mutants.........................................................................................................41

1.15 Souffle encodes zebrafish homolog of SPASTIZIN......................................................42

1.16 Souffle is maternally expressed ............................................................................................45

1.17 Electron microscopy in oocyte shows endosome accumulation .................................47

1.18 Souffle mutataion causes hereditary spastic paraplegia in human. .........................48

1.19 Souffle interacts with AP5 adaptor complex in human ...............................................50

1.20 Objectives of this study ….......................................................................................................52

2. MATERIALS AND METHODS............................................................................................53

2.1 Model Organism-Zebrafish ......................................................................................................53

2.2 Bacteria.................................................................................................................................53

2.3 Cell lines ....................................................................................................................................53

2.4 Chemicals, Buffers and Media ..................................................................................53

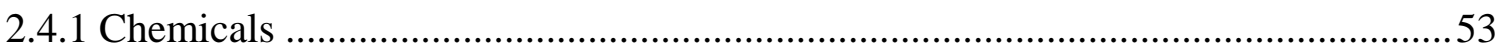

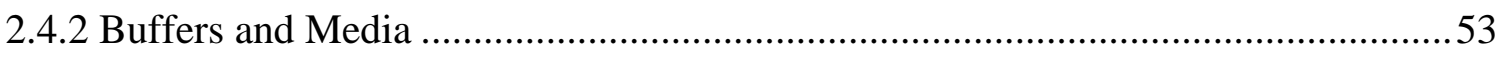

2.5 Constructs ...................................................................................................................................58

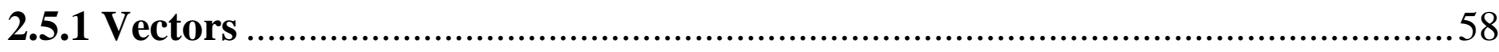

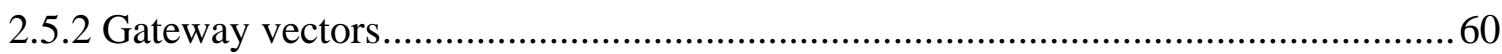

2.6 Oligonucleotides.......................................................................................................60

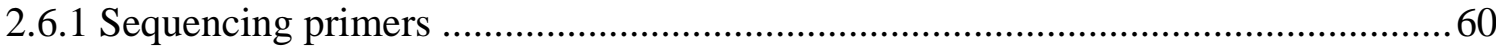

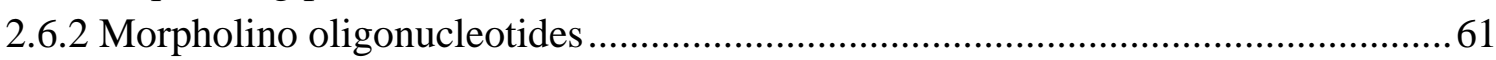

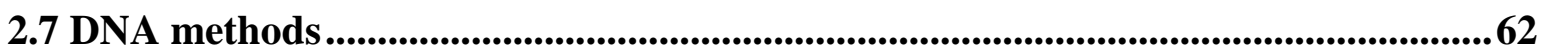

2.7.1 Plasmid DNA preparations ...........................................................................62 


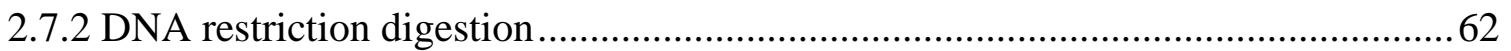

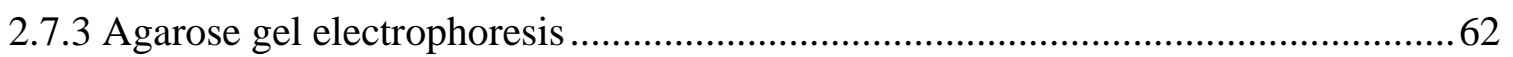

2.7.4 Purification of DNA fragments from agarose gel or restriction digestion mixture.63

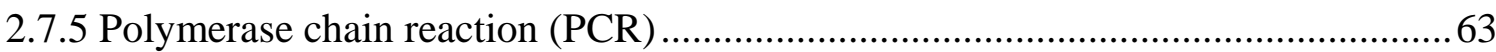

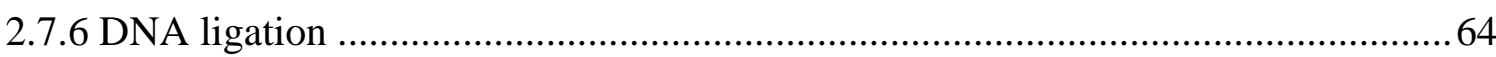

2.7.7 Chemical transformation and electro-transformation of bacterial cells....................64

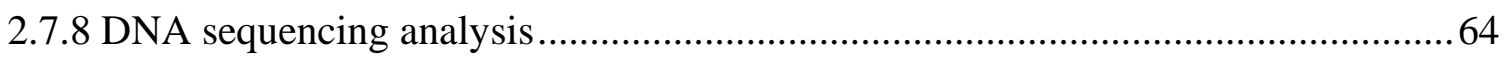

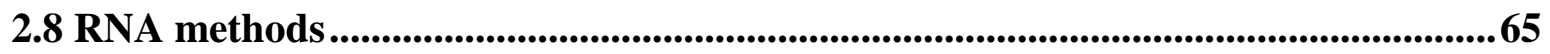

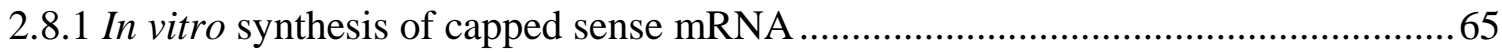

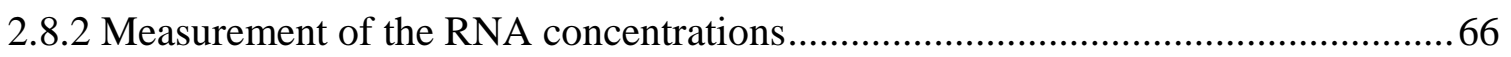

2.9 Zebrafish embryo culture and microinjections ....................................................66

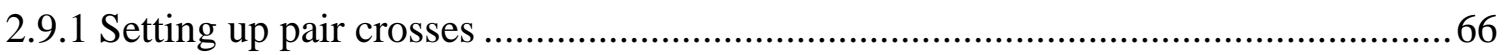

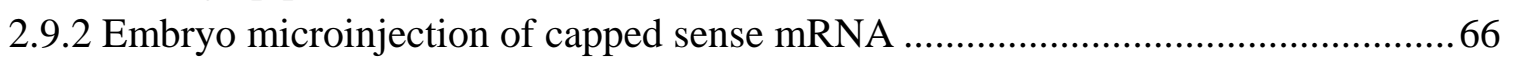

2.10 Oocyte methods..................................................................................................................................667

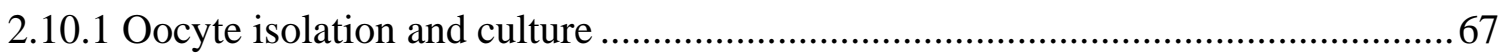

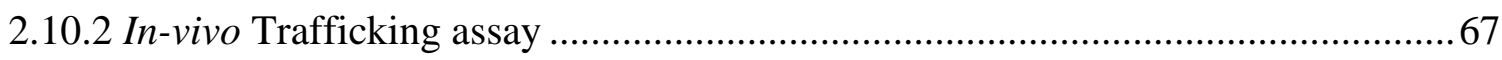

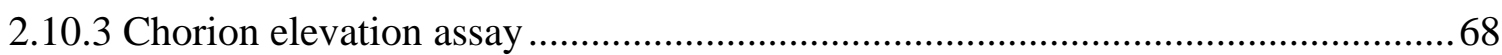

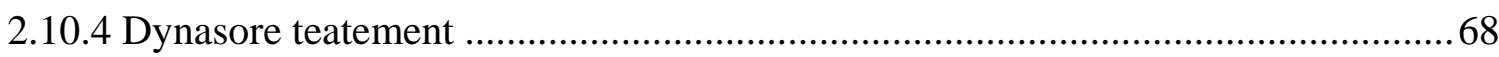

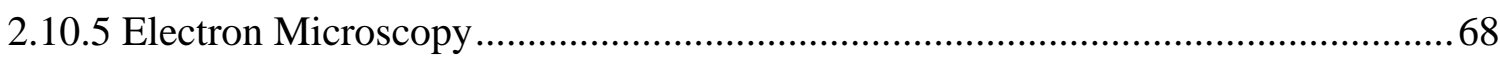

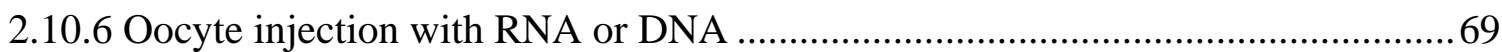

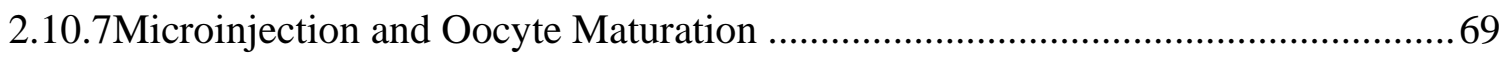

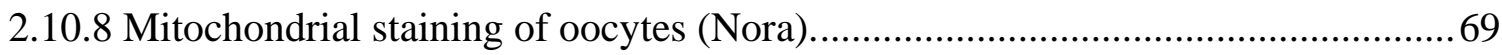

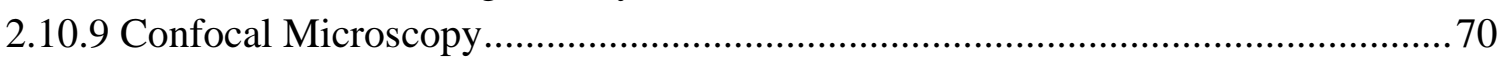

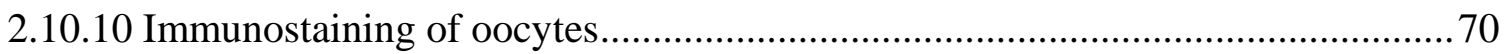

2.11 Motor neuron staining of zebrafish larvae.......................................................74

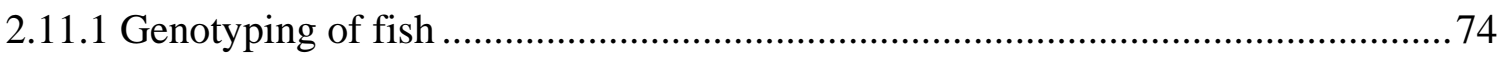

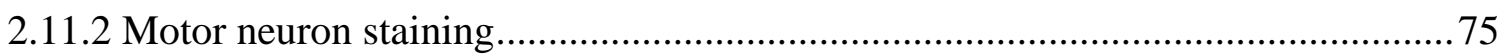

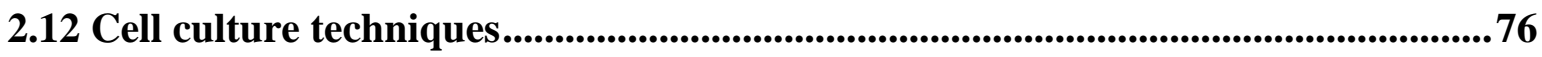

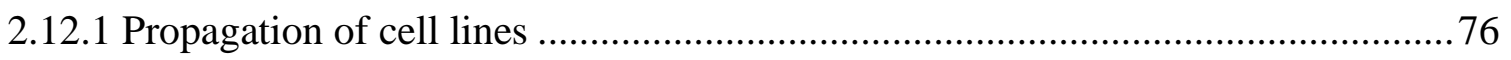

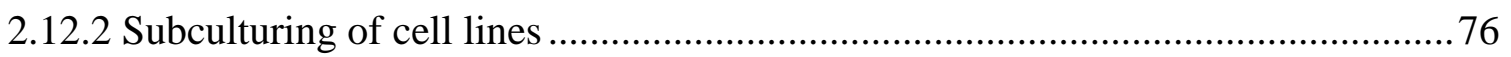

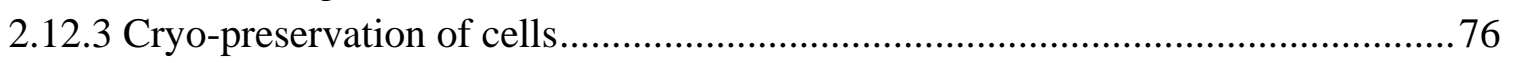

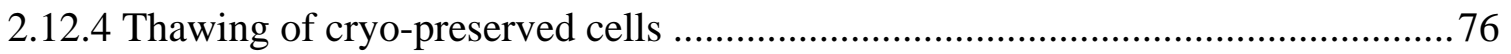

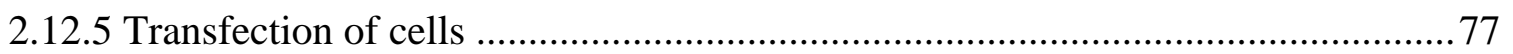

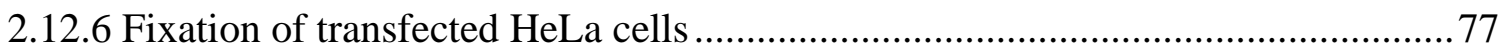

2.12.7 Immunofluorescent staining of cells (Susanne Schlick)........................................ 77

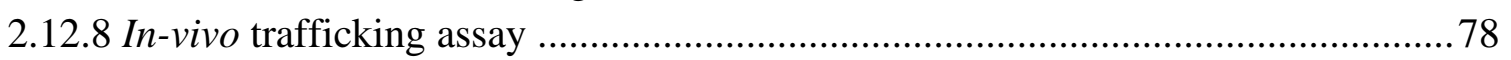

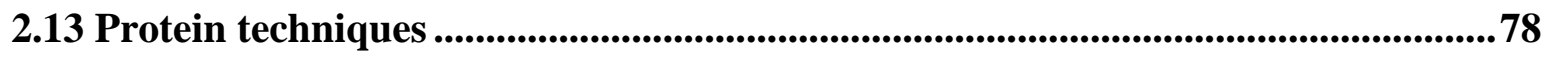

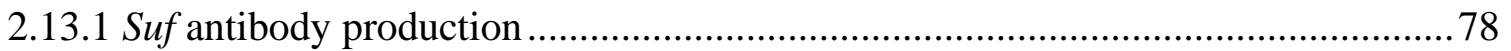

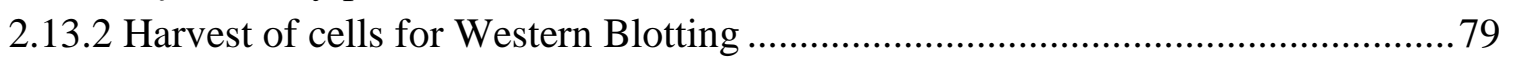

2.13.3 Protein electrophoresis under the denaturating conditions (SDS-PAGE) .............79 
2.15 Bio-informatic analysis

3. RESULTS.

3.1 Soufflé localizes to vesicles 82

3.2 Soufflé mutation produces truncated Suf protein .83

3.4 Suf mutants oocytes show endo-lysosomal defect.................................................84

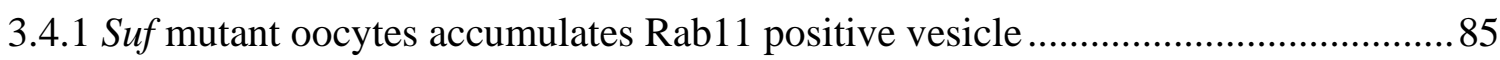

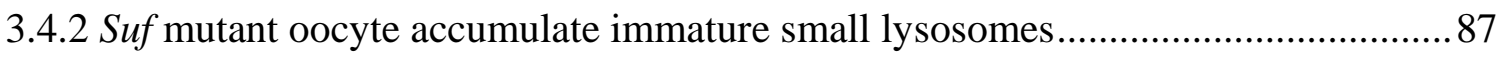

3.5. Recycling endosome is not affected in the suf mutant .............................................89

3.6 Suf localizes to Rab5, Rab11 positive endosome and lysosome................................90

3.7 Role of Suf on Rab11 positive vesicles ...................................................................91

3.7.1 Rab11 and Suf localizes to Secretory granule (Cortical Granule) ..........................91

3.7.2 Secretory granule accumulates in $s u f$ mutant oocytes ....................................... 95

3.8 Suf mutant failed to elevate the chorion..................................................................96

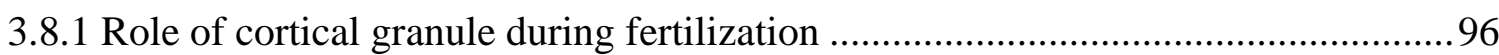

3.8.2 Suf mutant eggs fail to elevate the chorion after activation ......................................96

3.8.3 Re-organization of F-actin did not happen in suf mutant. ....................................98

3.9 Suf regulates secretory granule maturation into dense-core vesicle (DCV) ..........100

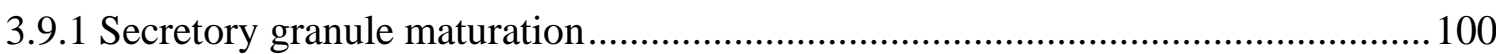

3.9.2 Suf mutant accumulates immature secretory granule (ISG) ............................... 100

3.10 Suf is necessary for vesicle fission from immature secretory granule (ISG) during secretory granule maturation ....................................................................................102

3.10.1 Suf mutant accumulates cortical compartment with clathrin buds ....................... 102

3.10.2 Dynamin accumulates on secretory granule in suf mutant ................................ 103

3.11 Chemical inhibition of Dynamin function mimics suf mutant phenotype ..........105

3.11.1 Inhibition of Dynamin obstructs chorion elevation in wildtype eggs................... 106

3.11.2 Dynasore treated eggs phenocopy soufflé mutant at molecular level ................. 106

3.12 Suf is required for mitosis but not for meiosis......................................................107

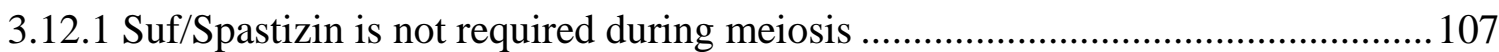

3.12.2 Soufflé embryos show defect in cytokinesis ...................................................... 109

3.13 Injecting suf DNA in the mutant oocyte rescues the mutant phenotype.............110

3.13.1 Suf DNA injection rescues chorion elevation phenotype .................................. 110 
3.13.2 Suf gene injection also rescues the molecular phenotype 111

3.13.3 Soufflé regulates cortical granule/ secretory granule maturation..... 112

3.14 Role of soufflé in lysosomal biogenesis and function.........................................113

3.14.1 Suf mutant shows defect in yolk degradation .................................................. 114

3.14.2 Suf mutant shows defect in lysosomal maturation........................................... 114

3.15 Yolk endocytosis is not affected in suf mutant................................................115

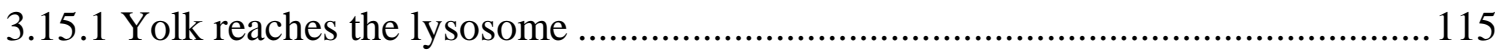

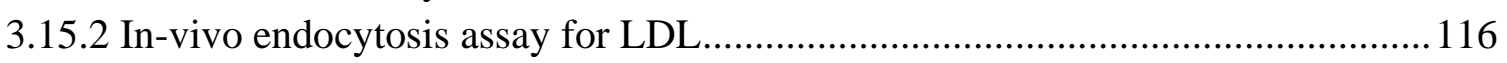

3.16 Vitellogenin processing enzyme Cathepsin D is not affected in suf mutant........117

3.17 Yolk protein degrading cathepsins are not delivered to lysosomes in the suf

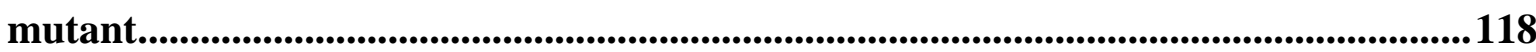

3.17.1 Cathepsin B, F and L not delivered to lysosome …...................................... 118

3.18 Lysosomal receptors M6PR and Sortilin transported correctly .............................120

3.19 Endo-lysosomal sorting adaptor protein complexes (AP1 \&GGA1) ...................122

3.20 Lysosomal membrane proteins (LAMP1 and V-ATPase) ......................................122

3.21 LAMP carrier pathway is affected in suf mutant ............................................124

3.22. Blocking secretion causes opaque egg phenotype.....................................................127

3.23 Bioinformatics analysis of Soufflé and VPS41 protein .......................................128

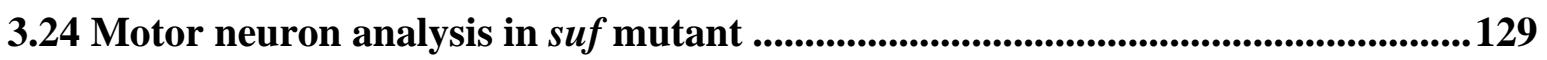

3.25 Suf protein knockdown by morpholino shows defect in notochord formation ..132

3.26 Analysis of SPG15 mutant patients fibroblast .................................................133

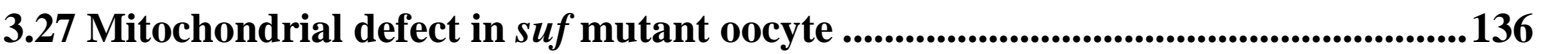

3.28 Cloning other opaque egg mutants ..........................................................137

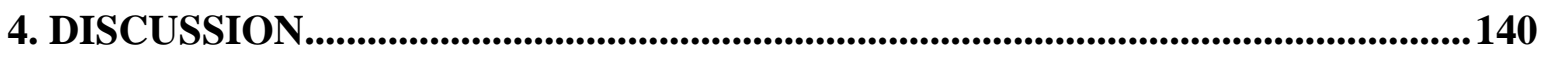

4.1 Souffle regulates Rab11b positive secretory vesicle. .....................................................140

4.2 Souffle localizes to secretory granule and endosomal compartment.......................141

4.3 Suf regulates secretory granule maturation. ............................................................... 142

4.4 Suf is essential for early mitotic cytokinesis..............................................................145 
4.5 Souffle is necessary for lysosomal biogenesis and function. ..................................146

4.6 Suf transport cathepsin proteases enzyme to lysosome. ........................................148

4.7 Acidification is required for DCV formation and Yolk degradation ....................153

4.8 Souffle regulate VPS41 mediated LAMP carrier pathway .................................155

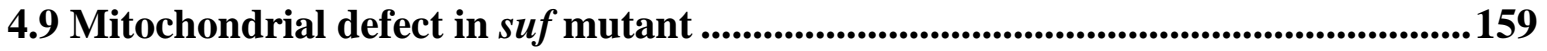

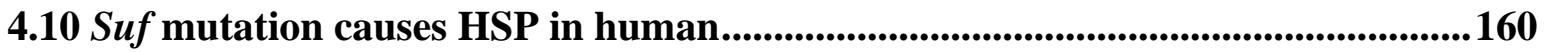

4.11 Proposed model for Suf functions in secretory granule and lysosome ................162

4.12 Opaque egg mutants might provide as a novel HSP candidate genes. ................163

4.13 Suf mutant is fitting model for HSP and vesicle trafficking..............................164

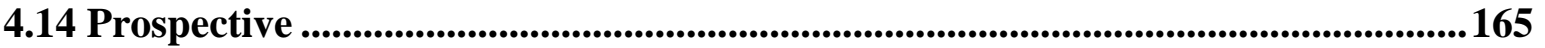

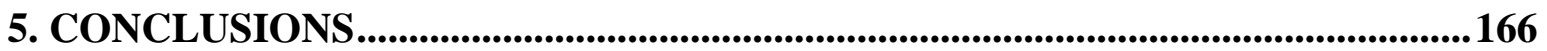

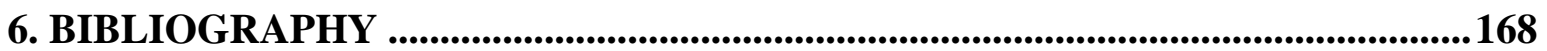

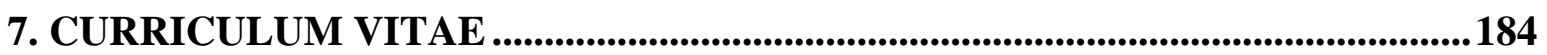




\section{Acknowledgements}

First of all, I would like to thank my supervisor Dr. Roland Dosch for giving me this project to work on and for all the guidance, which I have got during my work in his group.

I would like to thank Prof. Tomas Pieler for allowing me to perform my thesis in his department and the members of my thesis committee, Prof. Andreas Wodarz and Prof. Mikael Simons for the helpful discussions and advices I got during our meetings.

I would like to thank Prof. Joerg Grosshans for allowing me to utilize the confocal facility.

I also would like to thank our colloborators Dr. Joan Cerda and Dr. Dietmar Riedel.

I would like to acknowledge the work of rotation students (Christoph, Nora Crescata, Susanna Schlick, Ankit and Eva Dumann (Oxford, UK)).

I would also thank Dr. Stefan Höning (Koeln), Carlo Follo (France), Dr. Michael Schwake (Beilefeld) and Dr. E.J.R. Jansen (Netherlands) for providing antibodies.

I would like to thank Vinod and Pritesh for helping me in correcting my thesis.

I would like to thank all my colleagues from the fish group and Developmental Biochemistry team for the help and the wonderful atmosphere in the lab. Special thanks to Martina, Sai and Priyanka for helping me in tissue culture.

I would like to thank the DAAD and GGNB excellence fellowship for funding my studies at the Göttingen University.

My special thanks go to my parents and friends for all the care and support. 


\section{Abstract}

Successful reproduction of all sexually reproducing organisms mainly depends on the viability of the oocyte. The making of the oocyte is regulated at multiple level to ensure its quality where it carries the information from mother to the next generation. During oogenesis, apart from meiotic maturation cytoplasm also changes dynamically. The cellular transport pathways play an essential role in importing molecules from the mother and positioning them, which creates the polarity, axis and germplasm for future embryo. Vesicle trafficking is a vital transport process in all cells to import and deliver molecules to interact with the surrounding environment. This cellular mechanism is necessary for many biological processes like signal transduction, cytokinesis, polarity and lysosomal degradation. The discovery of many regulators (Rabs and SNAREs) in unicellular organisms showed the requirement of tight regulation in vesicle fission and fusion process. However, a forward genetic screen for yolk endocytosis in C.elegans discovered many novel regulators and a novel compartment, which are specific to multi-cellular organisms suggesting the presence of more complex regulation in vertebrates. Hence, finding new regulators are necessary to understand the process in vertebrates.

We aimed at identifying novel, vertebrate regulators of oogenesis in zebrafish oocytes using a maternal-effect mutagenesis screen. We isolated a group of mutants, which lays opaque rather than transparent embryos. One of the mutants named soufflé (suf) has been mapped to a FYVE- Zinc finger protein by positional cloning. This FYVE domain binds to PI3P lipids, which are predominantly found on endosomal membranes. Biochemical analysis revealed that these mutants have a defect in yolk cleavage during oocyte maturation, which leads to the observed opaqueness (Dosch et al., 2004). Furthermore, electron microscopy analysis revealed that suf accumulates smaller endosomes, whereas in wild types they mature into functional yolk globules in the oocyte. These preliminary results raised the question that how does soufflé regulate trafficking during oogenesis and what is the molecular mechanism behind the defect in suf mutant. Endosomal analysis showed accumulation of Rab11 and fragmented lysosome. In-vivo trafficking assay showed no defect in endocytosis and endosomal recycling. Rab11 localizes to secretory granule and suf mutant accumulates immature secretory granule (ISG). In-vivo assay showed that suf fail to elevate the chorion after fertilization indicating a defect in exocytosis of dense core vesicle (DCV). Further analysis showed that suf mutant accumulate secretory granule without dense core resulted from defective sorting and vesicle fission from ISG. Surprisingly, we discovered the vesicle in cortical region with 
many clathrin buds without fission accumulating in suf mutant using electron microscopy, consistent with accumulation of ISG marker VAMP4. Remarkably, chemical inhibition of dynamin function in the wild-type egg mimics suf mutant phenotype in cellular level and failed in chorion elevation. In addition, mis-organised F-actin showed the failure in exocytosis of vesicle. Rescue analysis with mutant construct showed that suf allele is a hypo morph. These results show that suf is necessary for sorting and fission during DCV formation.

Sorting from ISG during DCV formation is also necessary for lysosomal biogenesis. We found that suf mutant lysosome did not receive cathepsin B, F and L, yolk-degrading proteases, explaining the opaque phenotype. Furthermore, vATPase subunit d1 is also missing in the vesicle of suf mutant oocytes. Analysis of lysosomal sorting receptors and adaptor revealed that suf mutant does not have a defect in M6PR and sortilin mediated pathway, but they show reduced LAMP carrier intermediate mediated by VPS41 and AP3. In addition, AP3 positive vesicles are reduced while AP3 accumulates more on secretory granule consistent with reduced VPS41 recruitment on secretory granule. VPS41-AP3 mediated lysosomal transport carries lysosomal enzymes, SNAREs and other membrane proteins. The VPS41 and AP3 mutant phenotype explains the reason for fragmented lysosome and failure in DCV formation phenotype in suf mutant. Bioinformatics prediction shows that suf has a clathrin heavy chain domain, which can form clathrin like coat like in VPS41. Suf interaction with AP5 further suggesting that suf may work as a coat protein like VPS41 for lysosomal transport. We discovered a novel function for suf in sorting of lysosomal cargo sorting from ISG results in successful DCV formation and lysosomal function, in the absence of suf both lysosome and DCV formation is affected.

Interestingly, souffle mutation has been identified to cause progressive motor neurodegeneration in human. This defect in DCV formation could be one reason for why the long motor neuron loses its connection and causes spasticity, since DCV is necessary for long-term potentiation. Hence, souffle mutant egg is an excellent model to study the molecular mechanism and disease pathology. 


\section{List of Figures}

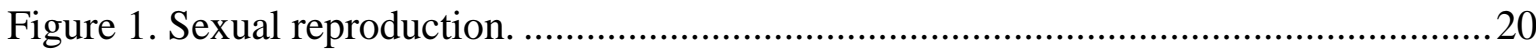

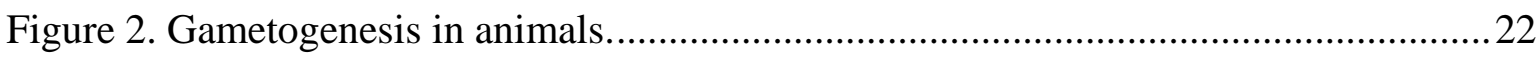

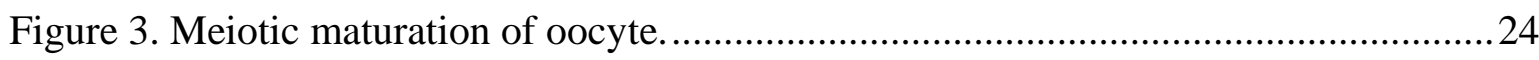

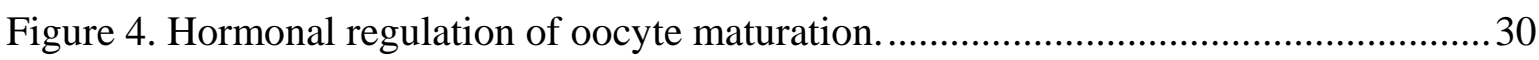

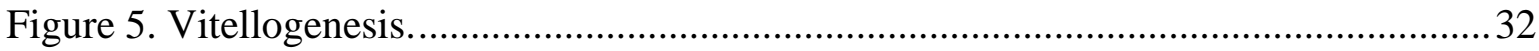

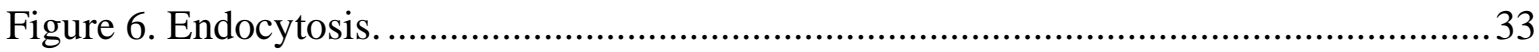

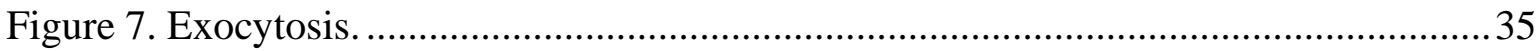

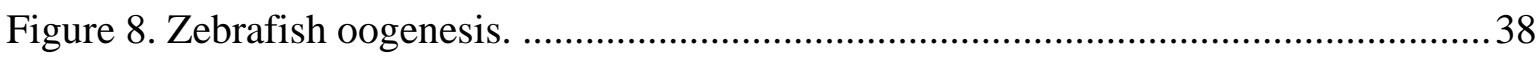

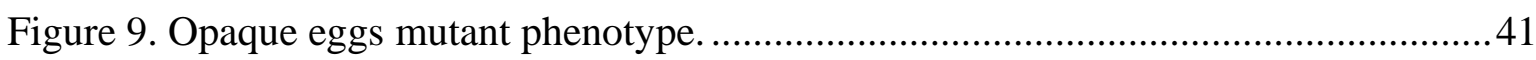

Figure 10. Yolk protein profile of opaque egg mutants. ................................................42

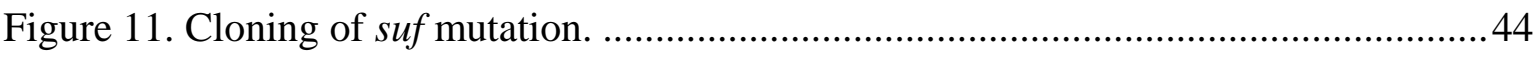

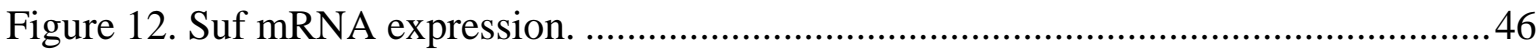

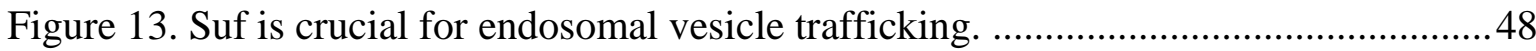

Figure 14. Pathogenic mechanisms involved in hereditary spastic paraplegia (HSP), .......49

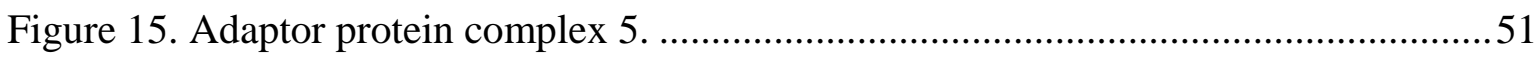

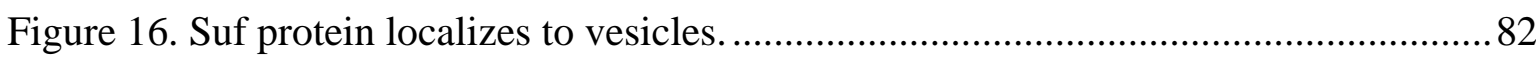

Figure 17. Suf p96re mutation creates truncated suf protein......................................... 83

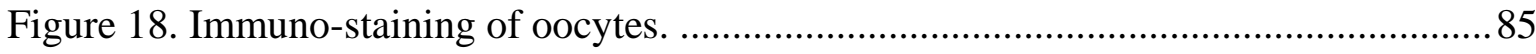

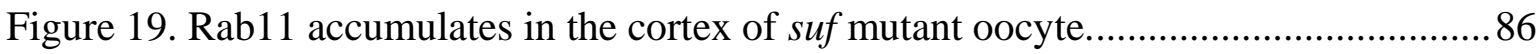

Figure 20. Rab11 and Rab7 accumulate in suf mutant oocytes........................................... 88

Figure 21. Tfn recycling is not affected in the $s u f$ mutant. ................................................90

Figure 22. Suf colocalize with Rab5, Rab11 and lysosome.............................................94

Figure 23. Rab11b and suf localizes to secretory granules in zebrafish oocyte..................95

Figure 24. Suf mutant accumulates secretory granule......................................................95

Figure. 25 Suf mutant fail to elevate the chorion. ..............................................................97

Figure 26. Suf mutant fail to reorganize the cortical F-actin.............................................99

Figure 27. Suf mutant oocyte accumulates immature secretory granule........................... 102

Figure 28. Suf mutant oocyte accumulates Clathrin buds on the vesicular compartment. .103

Figure 29. Suf mutant oocytes accumulate clathrin and Dynamin.................................. 104

Figure 30. Dynasore treated oocyte mimics soufflé phenotype. ..................................... 106

Figure 31. Dynasore treated egg mimics suf mutants functionally................................... 107

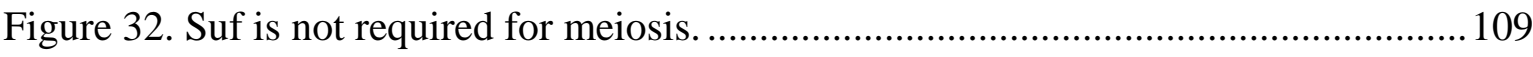


Figure 33. Suf mutant embryos shows a defect in cytokinesis...................................... 110

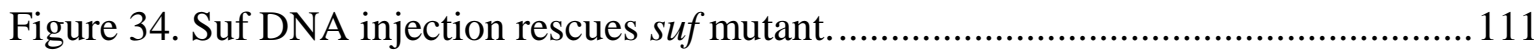

Figure 35. Suf DNA injection rescues the mutant phenotype at molecular level. ............. 112

Figure 36. Soufflé regulates sorting and fission during secretory granule maturation. .....113

Figure 37. Soufflé mutant lays opaque egg. ............................................................ 114

Figure 38. Soufflé mutant shows defect in yolk degradation............................................ 114

Figure 39. Yolk reaches the lysosome in suf mutant oocyte........................................... 116

Figure 40. Cathepsin D protease reaches the lysosome in suf mutant oocyte.....................117

Figure 41. Cathepsin B and L are not delivered to the lysosome in suf mutant oocyte. .... 118

Figure 42. Cathepsin $\mathrm{F}$ is not delivered to the lysosome in suf mutant oocyte................... 120

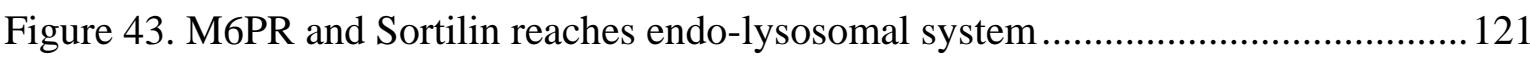

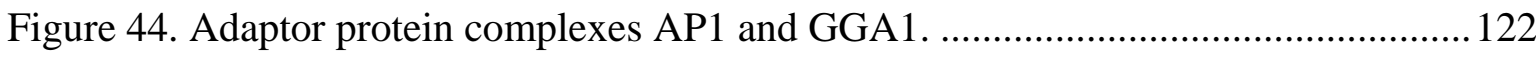

Figure 45. LAMP carriers are reduced in suf mutant oocytes.......................................... 123

Figure 46. V-ATPase subunit d1 does not arrive in suf mutant lysosome. ......................... 124

Figure 47. VPS41 positive Lamp carriers are reduced in the suf mutant..........................126

Figure 48. AP3 accumulates in secretory granule in suf mutant..................................... 127

Figure 49. Delaying secretion inhibits lysosomal function.............................................. 127

Figure 50. Suf protein predicted to have Coat protein sub unit and CHC like VPS41......129

Figure 51. Motor neuron analysis of suf mutant fish. .................................................... 131

Figure 52. Suf morpholino knockdown shows defect in notochord lysosome. ..................132

Figure 53. Analysis of spg 15/spastizin mutant fibroblast cells........................................ 134

Figure 54. Suf mutant show disturbed mitochondrial network. ........................................ 136

Figure 55. Other opaque egg mutants also show fragmented lysosome. ...........................139

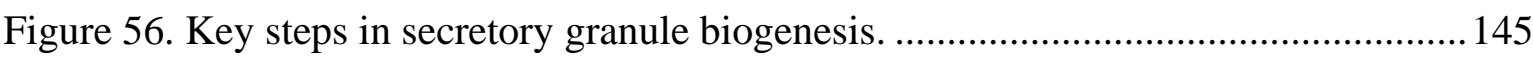

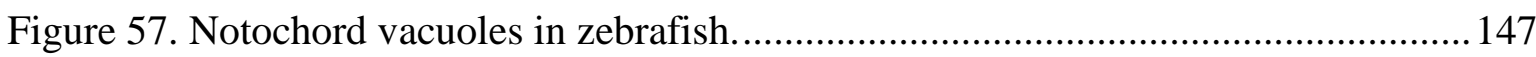

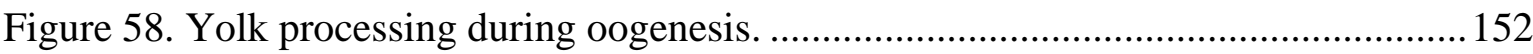

Figure 59. Morphological and ultra-structural changes of yolk inclusions during oocyte

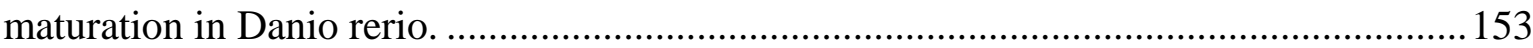

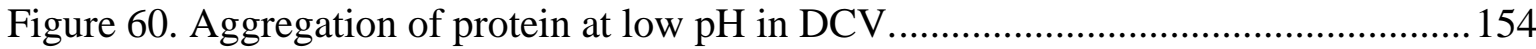

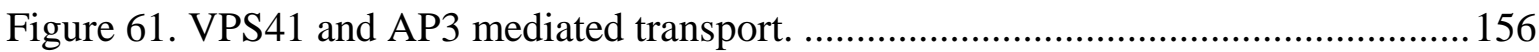

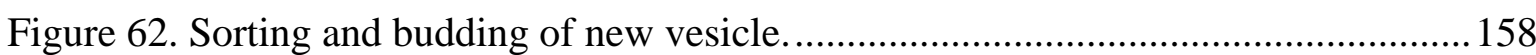

Figure 63. Mitochondrial connections to lysosome and ER. ........................................... 160

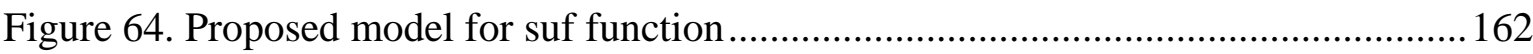

Figure 65. Suf mutant oocyte is a fitting model for HSP. .............................................. 165 


\section{List of Tables}

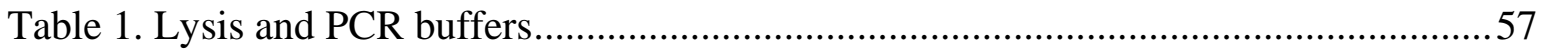

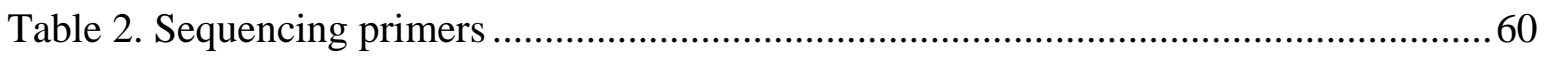

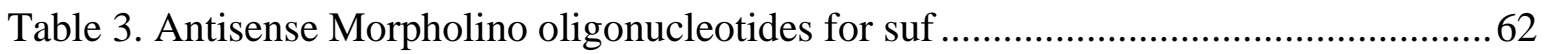

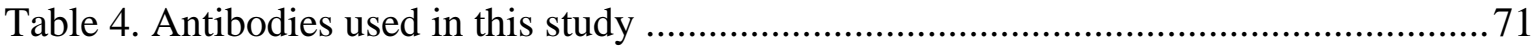

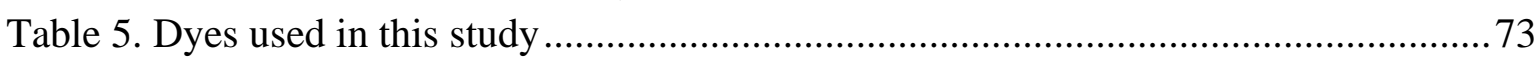

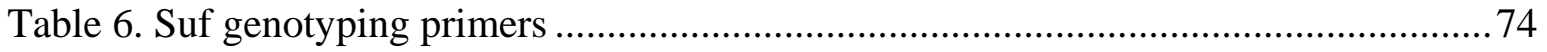

Table 7. PCR reaction setup for genotyping suf ......................................................... 75

Table 8. PCR program for suf genotyping ................................................................ 75

Table 9. The sizes of separated proteins and the percentages of the correspondent acrylamid

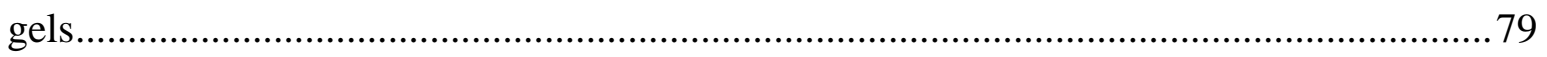




\section{Abbreviations}

Suf, Souffle

GFP, Green Fluorescent Protein

CFP, Cyon Fluorescent Protein

DMSO, Dimethylsulfoxide

hCG, human chorionic gonadotropin

LB, Luria-Bertani

PAGE Polyacrylamide gel electrophoresis

PCR, Polymerase chain reaction

PGCs, Primordial germ cells

RT-PCR reverse transcriptase Polymerase chain reaction

SDS, sodium dodecyl sulfate

Q-PCR, Quantitative Polymerase chain reaction

RT-PCR, reverse transcriptase Polymerase chain reaction

SAP, Shrimp alkaline phosphatase

SOB and SOC, Super Optimal Broth

SSLP, simple sequence length polymorphism

$\mathrm{pH}$, negative decade logarithm of hydrogen ion concentration

PBS, phosphate buffered saline

MEM, MOPS-EGTA-MgSO4 buffer

MEMFA, MOPS-EGTA-MgSO4 formaldehyde buffer

min, minutes

$\mu$, micro

m, milli

M, molar

k, kilo

$\mathrm{kb}$, kilobase

1 , liter

DCV, Dense Core Vesicle 
iDCV, Immature Dense Core Vesicle

mDCV, Mature Dense Core Vesicle

LDCV, Large Dense Core Vesicle

LDL, Low Density Lipoprotein

LDLR, Low Density Lipoprotein Receptor

ISG, Immature Secretory Granule

MSG, Mature Secretory Granule

POMC, Proopiomelanocortin

CPE, Corbaxy peptidase Y

AP, Adaptor protein complex

HSP, Hereditary Spastic Paraplegia

GVBD, Germinal Vesicle Break Down

MPF, Maturation Inducing Factor

LH, Lutinising Hormone

FSH, Follicle Stimulating Hormone

DES, Dieethyl stilbestrol

CREB, cAMP response element-binding protein

PVS, Peri Vitellin Space

ERC, Early Recycling Compartment

hpf, hour post fertilization

EM, Electron Microscopy

SAC, Sub Apical Compartment

ER, Endoplasmic Reticulum

TGN, Trans Golgi Network

M6PR, Mannose 6-Phosphate Receptor

BDNF, Brain Derived Neurotropic factor

ALP, Alkaline Phosphatase Pathway

CHC, Clathrin Heavy Chain

ERMES, ER and Mitochondria encounter structure

vCLAMP, Vacuole and Mitochondria patch 
Abbreviations

MPA, Maclura pomifera agglutinin

DAPI, 4',6-diamidino-2-phenylindole

VTG, Vitellogenin

YP, Yolk Protein

LTP-LongTermPotentiation 


\section{Introduction}

\subsection{Reproduction}

Reproduction is essential process for any species to propagate and sustain. Reproduction is a biological process where the offspring is produced from their parents. Among the two types of reproduction, most prokaryotes reproduce by asexual method by fission in bacteria while the eukaryotes uses sexual reproduction. Sexual reproduction is the primary method for propagating ones genes in almost all animals and plants. The sexual reproduction involves two main steps, Meiosis where the chromosomal numbers are reduced to half $(n)$ and then fertilization where the gametes from two parents fuse and restore the original number of chromosomes (2n) (Fig.1). During these two processes, the DNA is exchanged by recombination between the homologoues pairs and which is thought to adapt to the new environment, remove the mutations and competitions.

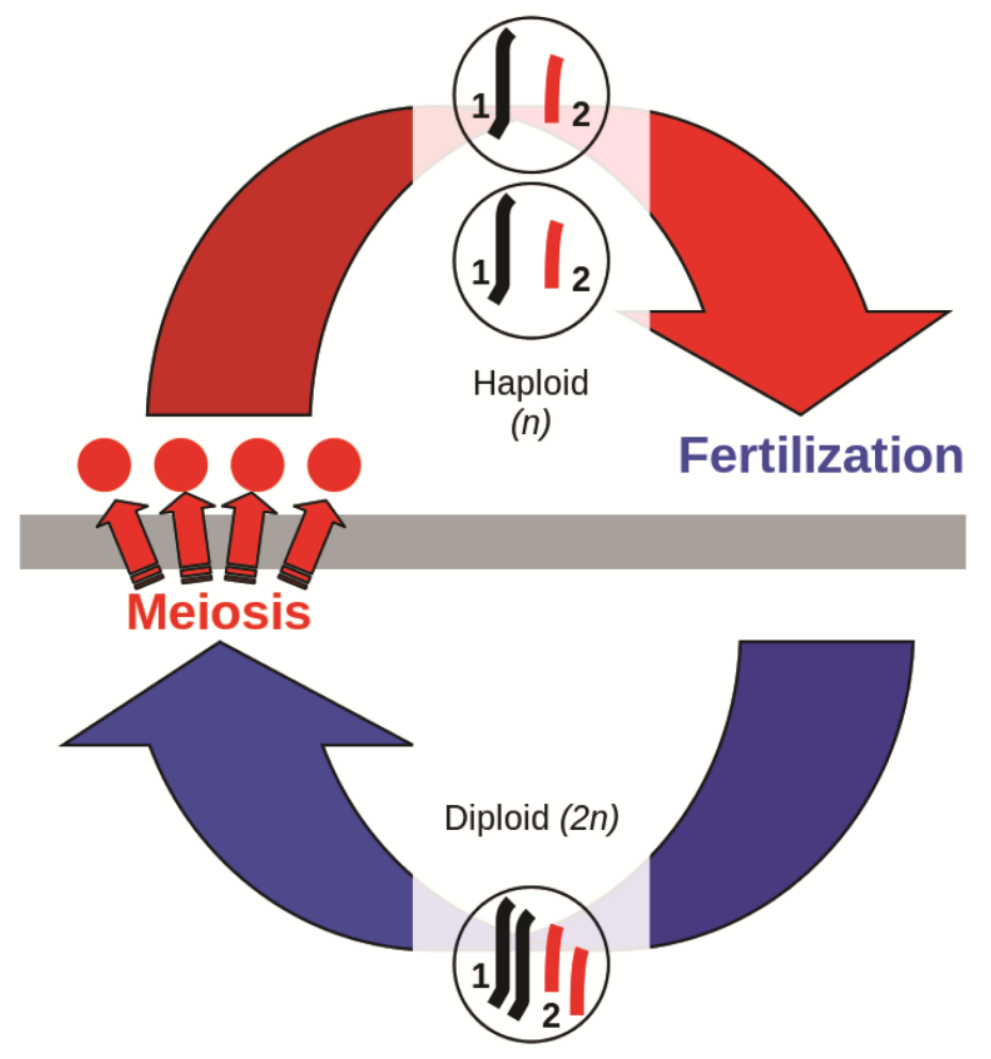

\section{Figure 1. Sexual reproduction.}

Cartoon shows the division in DNA during meiosis and addition during fertilization. (http://en.wikipedia.org/wiki/Sexual_reproduction) 


\subsection{Gametogenesis}

The success of sexual reproduction mainly depends on the viability of the two gametes from the parents carrying haploid chromosomes. Gametogenesis is a process of gamete formation from primordial germ cells (PGC). The two processes of gametogenesis, which are spermatogenesis and oogenesis, vary in many ways in producing gametes from PGC. The spermatogonium can produce four functional sperm from each cell while the oogonium will generate one ovum by removing just polar bodies during the two meiotic division processes to accumulate maternal components (Fig. 2). The spermatogenesis is a continues process whereas the oogenesis starts even before birth and blocked in prophase I until it receives the ovulation signal later. The contribution from sperm is mainly the DNA and centrosome while the ovum brings most of the factors, which are necessary during oogenesis like, food and genetic factors that are required for early embryonic development. Hence, production of viable and quality egg is crucial for sexual reproduction and is an energy consuming process.

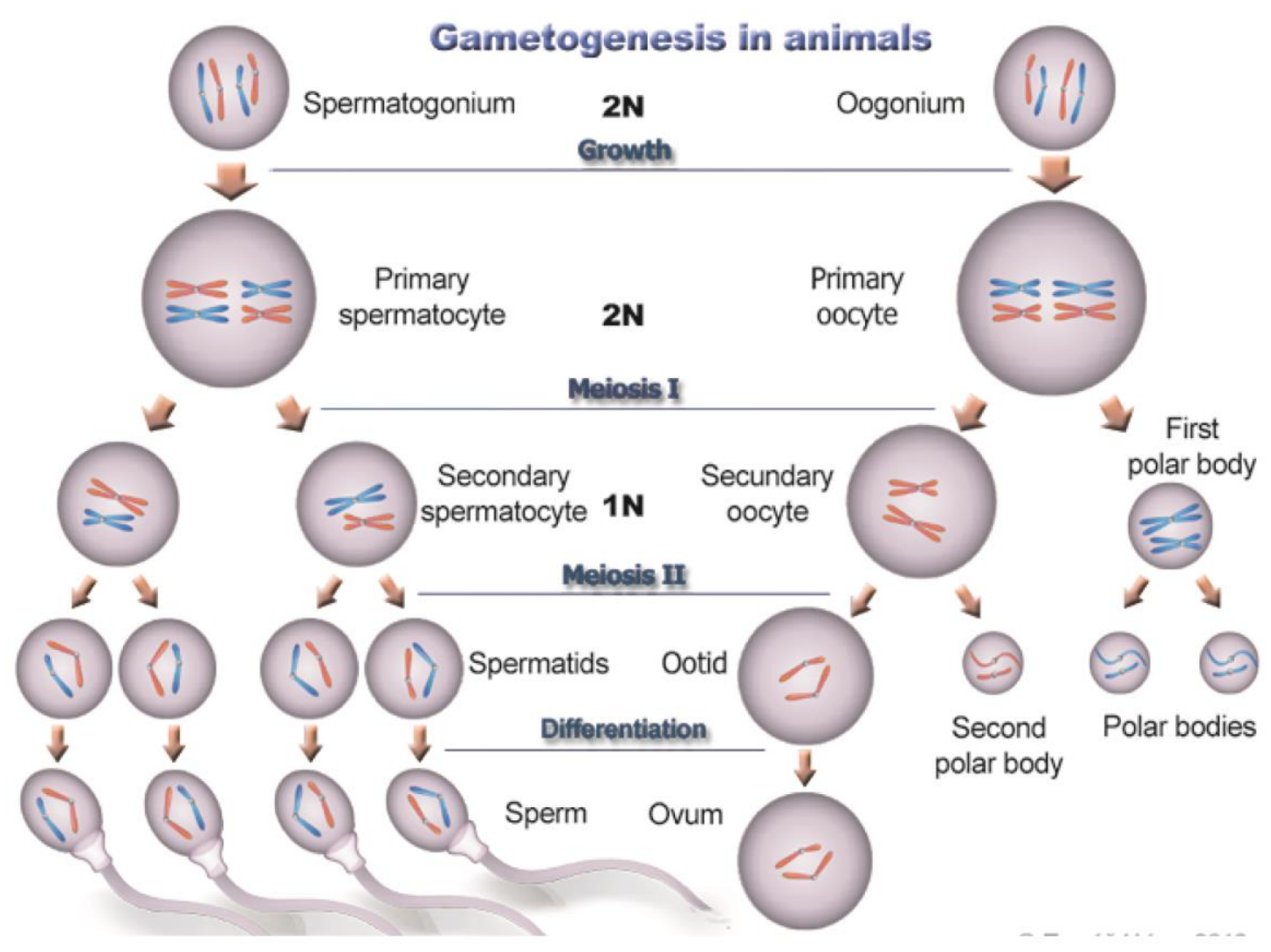




\section{Figure 2. Gametogenesis in animals.}

The picture shows the DNA content reduction in spermatogenesis and oogenesis. The process of making sperm and oocyte from their progenitors

(http://web2.mendelu.cz/af_291_projekty2/vseo/stranka.php?kod=285).

\subsection{Ovary}

The ovary is one of the most complex organ, both structurally and physiologically with many different somatic and follicle cells containing different stages of developing oocytes. It is amply supplied with connective tissue, blood vessels, nerves, and smooth muscle and is surrounded by a surface (coelomic) epithelium and its stroma is differentiated into a cortex (with developing follicles) and a medulla (with blood vessels). The ovary is a major endocrine organ interacting with the pituitary gland and the uterus during each menstrual cycle and is regulated by gonadotropins and steroid hormones (Sathananthan AH. et al, 2006). The ovary plays two main roles in female reproductive system by acting as both glands and gonad. As a gland, it produces estrogen and progesterone, which are necessary for reproduction. As a gonad, ovary holds the follicle cells for the whole lifetime and few start to develop into an oocyte during menstrual cycle but one finally mature into ovum.

\subsection{Oogenesis}

The egg is the supreme precursor of the newly developing embryo. Therefore, the making of a viable egg is the most crucial event for sexual reproduction. Oogenesis is a very dynamic process in the ovaries, in which the oocyte passes through various phases of development. The oogonia differentiate from the PGC and the ovary provides nests of oogonia associated with developing follicle cells in the cortex. Every oogonium is surrounded by follicle cells and they are connected to the developing oogonium. Oogonia have vesicular nuclei with 1-3 nucleoli and small cytoplasm for few cellular organelles. The oogenesis consists of two phase oocyte growth and oocyte maturation.

\subsubsection{Oocyte growth}

\subsubsection{PGC to Oogonium}

Animal kingdom has two kinds of diploid cells, one is somatic where they develop into a whole organism and stay diploid while the germ cell goes through reduction division to form gametes for reproduction (Wallace and Selman, 1981 \& Selman et al., 1993). The 
gradual transformation of PGC into oogonium follows with the increase in size of the cell and nuclei. The endoplasmic reticulum and Golgi are accumulated and 'nuages' of mitochondria are numerous and bigger. The cell starts to divide faster mitotically and surrounded by pre-granulosa cells. The cytoplasm contains few ribosomes and reduced electron density in the electron micrograph compared to PGC (Brusle, 1982).

\subsubsection{Oogonium to primary oocyte}

The diploid oogonia after number of mitotic divisions undergo their first meiotic division to differentiate into primary oocytes. Initially, the oogonium carries two copies of the chromosomes from both parents. During the interphase after the mitotic division, the cell prepares for its first meiotic division. DNA replication happens during this stage and produce sister chromatids for RNA synthesis which is specific for primary oocyte (Grier, 2000). Once the meiotic division is initiated, then they are called primary oocyte, which is surrounded by theca cells and granulosa cells constituting ovarian follicle. Granulosa cells are specialized somatic cells around oocyte, which secrete basement lamina (Nagahama, 1997). The development of the primary oocyte stage is divided into four stages. Stage I oocytes are characterized by formation of chromatids. During stage II or pre-vitellogenesis stage, the molecules required during later stages are synthesized and yolk precursors start to enter. Furthermore, the newly synthesized RNP granules accumulate. Stage III or vitellogenesis stage is characterized by accumulation of yolk, lipids and formation of zona radiata $(\mathrm{ZR})$. Stage $\mathrm{IV}$ is the maturation process where the first meiotic division is completed. This first meiotic division remains arrested at the end of prophase stage until they get the next signal to proceed. During this period, the oviparous animals accumulate essential components and nutrients for the embryo development by a process called vitellogenesis. At the end of vitellogenesis, the primary oocyte resumes their first meiotic division and continues maturation.

\subsubsection{Primary oocyte to secondary oocyte}

During stage IV the primary oocyte resume the meiotic division, undergoes germinal vesicle beak down (GVBD) followed by release of first polar body. Then the oocyte enters the second meiotic division without a break in interphase and being arrested in metaphase until fertilization (James E Ferrell, 1999). This maturation is induced by the hormone progesterone and can be divided into nuclear and cytoplasmic maturation. 
Meiotic Maturation
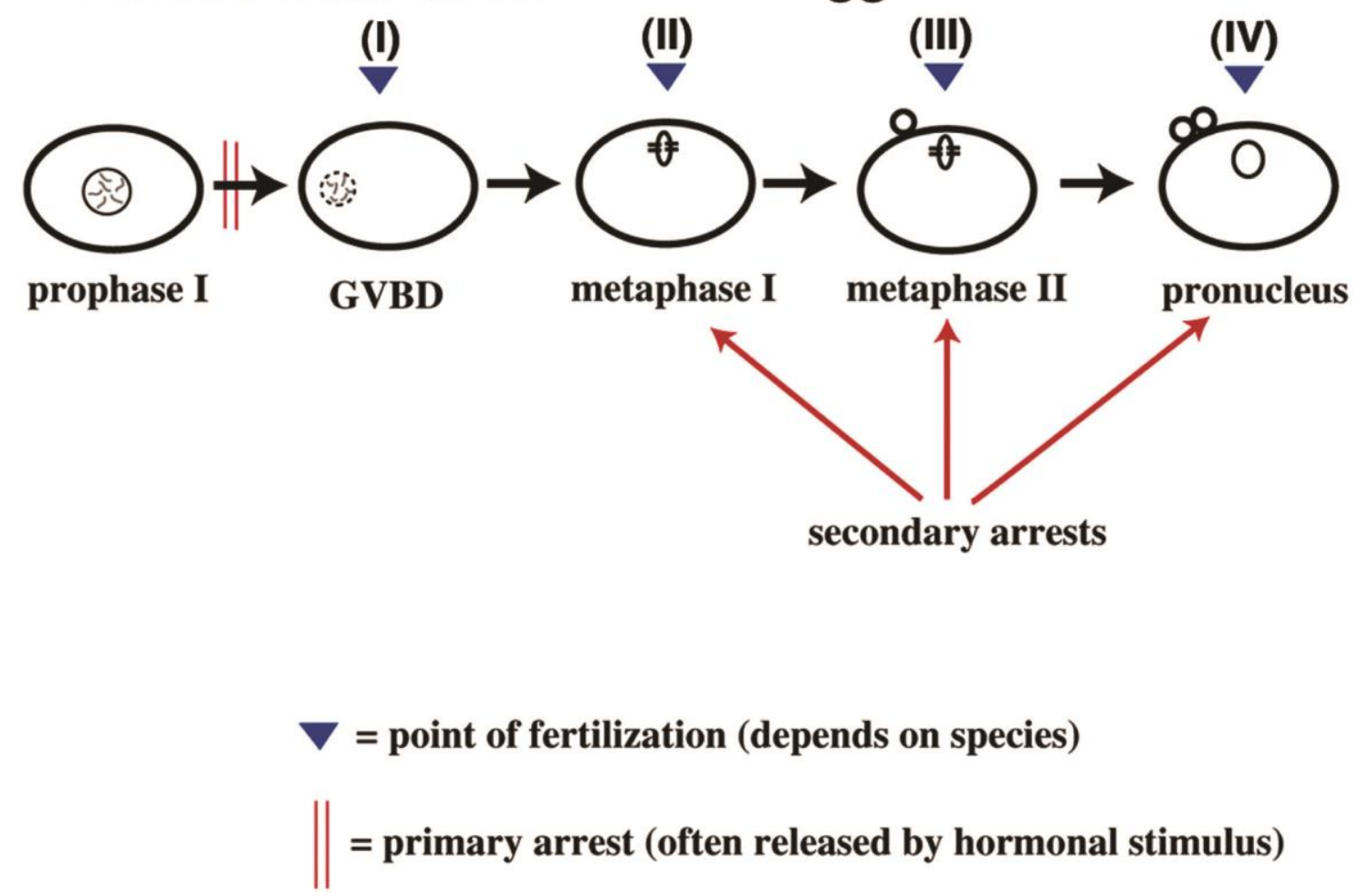

Figure 3. Meiotic maturation of oocyte.

Oocytes from most species undergo a first arrest at prophase I (PI) that is maintained for a few days (Drosophila) or for decades (humans). On hormonal or developmental stimulation, oocytes undergo meiotic maturation, release the primary arrest, and enter a second arrest at metaphase I (MI), metaphase II (MII), or post meiotic $\mathrm{G}_{1}$ depending on the species. Fertilization triggers the release from the second arrest and the completion of meiosis in vertebrates (Xenopus and mammals). Drosophila releases the secondary arrest in a sperm-independent manner. GV, germinal vesicle; PI, prophase I; GVBD, germinal vesicle breakdown; MI, metaphase I; PB1, polar body 1; MII, metaphase II; PB2, polar body 2.

\subsection{Oocyte maturation}

Oocytes gradually acquire nuclear and cytoplasmic maturation during growth. Meiotic competence, which is the capacity of the oocyte to resume meiosis and become nuclearly matured, is acquired during folliculogenesis. Developmental competence is related to cytoplasmic maturity of the oocyte and refers to the capacity of the oocyte to be fertilized and develop into a healthy embryo capable of continuing its development to term and producing a live birth. Cytoplasmic maturation is acquired after the oocyte becomes meiotically competent and involves an accumulation of transcripts and other factors (Flor Sánchez and Johan Smitz, 2012). 


\subsubsection{Nuclear / Meiotic maturation}

Nuclear maturation refers to the meiotic process of chromosomal reduction to a haploid content, so as to produce a diploid organism upon fusion with sperm. The oocyte achieves nuclear maturation when they are still developmentally not competent. This stage has to be paused until the cytoplasmic maturation is complete to develop further. This arrest is maintained by the oocyte by elevated levels of cyclic adenosine monophosphate (cAMP) produced by cumulus cells or endogenous production by the oocyte. Oocyte meiotic maturation involves a cascade of processes that is initiated with the pre-ovulatory LH surge, leading to the progression of the oocyte to the metaphase II stage and ending with the extrusion of the first polar body.

Oocyte meiotic maturation is defined by the transition between diakinesis and metaphase of meiosis I and is accompanied by nuclear envelope breakdown, where rearrangement of the cortical cytoskeleton and meiotic spindle assembly takes place (Fig. $3)$.

Before maturation starts, the oocyte contains a germinal vesicle (GV) with a large nucleolus. The chromosomes in the GV are mostly decondensed, dispersed, and transcriptionally active (reviewed in Smith and Richter, 1985; Wassarman, 1983). With the initiation of maturation, transcription ceases and, the chromosomes begin to condense, the GV breaks down, and nucleoli disperse (Masui and Clarke, 1979). As maturation progresses, the paired homologous chromosomes align in the middle of the forming meiotic spindle during metaphase I. Separation of the paired homologous chromosomes is followed by the first polar body formation. Then the chromosomes remaining in the oocyte are rearranged again on meiotic spindles at metaphase II. With the second meiotic division, chromatids separate and the second polar body is formed. Finally, the chromatids remaining in the oocyte decondense and a pronucleus forms (Fig.3). Both polar bodies persist during early cleavage in the PVS and will eventually degenerate. They are miniature eggs having some of the organelles found in oocytes. While PB1 has CGs, PB2 is usually devoid of or has few CGs. Recently, polar bodies have been used for pre-implantation genetic diagnosis. The PB1 can be penetrated by sperm and behave exactly like the oocyte by releasing its CGs (Sathananthan et al, 2006). PB1 could also cleave since it inherits a sperm centrosome. The meiotic cells of different organisms become fertilizable at different stages along the process of meiosis. For example, the eggs of most vertebrate species arrest at the second metaphase, and await fertilization to release them into embryonic 
development, while sea urchin eggs proceed through meiosis to completion, and are stored as cells with haploid pro-nuclei.

The timing of the meiotic divisions with respect to fertilization varies among species, likely reflecting the diversity of reproductive strategies observed in nature. Despite these differences in timing, extensive studies reveal striking conservation in the molecular underpinnings of oocyte meiotic maturation among different animals. It was classic studies of oocyte meiotic maturation in amphibian oocytes that led to the discovery of the Maturation Promoting Factor (MPF; Masui and Markert, 1971; reviewed by Masui, 2001). Genetic and biochemical analysis of the cell cycle, together with MPF purification, subsequently demonstrated that cyclin-dependent protein kinases are universal regulators of mitotic and meiotic cell cycle progression in eukaryotes (reviewed by Morgan, 1995).

\subsubsection{Cytoplasmic maturation of the oocytes}

Cytoplasmic maturation involves the cytoplasmic changes required to prepare the cell for fertilization, activation, and embryo development. In various model organisms, it includes acquisition by the female germ cell of competence to fuse with sperm, decondense sperm chromatin, form pronuclei, and prevent polyspermy.

The process of cytoplasmic maturation is accompanied by changes in cellular organelles. Oocytes possess a variety of organelles typical of most cells (such as Golgi apparatus, mitochondria, endoplasmic reticulum) as well as oocyte-specific (yolk globules, cortical granules). Some of these organelles do not undergo any transformations during maturation (mitochondria, yolk), while others (such as cytoskeletal elements, cortical granules, and endoplasmic reticulum) exhibit dramatic changes and are hallmarks of meiotic progression.

\subsubsection{Microtubules}

The execution of meiotic events depends on the oocyte cytoskeleton. The growing oocyte is characterized by elongated, labile cortical microtubules that undergo dramatic changes during oocyte maturation. Microtubules progressively disassemble in the cortex, and the meiotic microtubule spindle then forms in the maturing oocyte to mediate chromosome segregation during both divisions of meiosis. During meiosis, eggs of most animals lose their microtubule-organizing center or centrosome, so that they rely on fertilization and the parental centrosome for further cleavage. 


\subsubsection{Cortical Granules}

The cortical granules accumulated in the oocytes acquire the capacity for exocytosis during maturation. Their function is to secrete their contents at fertilization and modify the extracellular matrix of the egg, such that it does not support binding and fusion of extra sperm. It is critical for the cell to rapidly exocytose its cortical granules at fertilization to block polyspermy, but also to prevent precocious exocytosis of these cortical granules, otherwise the extracellular matrix would be irreversibly modified and the cell would not be receptive to sperm. The oocyte synthesizes and accumulates large numbers of cortical granules throughout oogenesis. In oocytes where cortical granules have been quantified, they reach 8,000 in mice and 15,000 in sea urchin. During oocyte maturation, cortical granules move to the periphery. This change in distribution is linked to the acquisition of the capacity of these granules to exocytose upon fertilization. Cortical granules rearrangements depend on de novo formation of microfilament during maturation.

\subsubsection{Actin filaments}

Microfilaments form the scaffold for organelle movement during oocyte maturation. Actin reorganization during maturation is not connected to GVBD although the function in GVBD is not clear. In vertebrate oocytes, actin polymerization appears to be required for the translocation of the meiotic spindle from the center of the cell to an asymmetric cortical location, and as a consequence for the first polar body extrusion. Recent work identifies the microfilament-binding protein formin as a necessary molecular regulator of this process, as the spindle does not migrate and polar bodies are not extruded in oocytes from formin knockout mice (Leader et al., 2002). The other transport event that actin microfilaments are required for is translocation of cortical granules (Wessel et al., 2002).

\subsubsection{Endoplasmic reticulum}

ER in many organisms undergoes significant change during maturation. The changes in the ER structure are of particular interest as this organelle releases calcium at fertilization mediating egg activation, and this ability to release calcium develops during oocyte maturation. Immature oocytes of all species studied possess relatively uniform three dimensional networks of ER tubules with some individual cisternae and annulate lamellae (accumulations of cisternae) deep in the cytoplasm (Babinnec et al., 2003). The detected changes of the ER in the course of oocyte maturation include reorganization, or formation of circular structures around the yolk platelets (Terasaki et al., 2001). Changes in the structure of the ER, especially dispersion of the nuclear envelope, and fragmentation of ER 
tubules, are due to the cell cycle progression, and have been documented for mitosis as well. However, several features of ER behavior are unique for meiosis. In maturing oocytes, the ER is not associated with the meiotic spindle, while such association is detected in mitotic cells. Furthermore, the formation of cortical clusters is specific for oocyte maturation and is ultimately required for formation of calcium release mechanisms in the egg at fertilization. These clusters disappear sometime after fertilization and are not detected in mitotic embryonic cells (FitzHarris et al., 2003).

\subsubsection{Mitochondria}

Mitochondria are the most prominent organelles in the ooplasm. They are predominantly spherical to oval with dense matrices and few arch-like or transverse cristae presenting an inert appearance. Some mitochondria may present clear vacuoles within their matrices and a few are extremely large ("giant mitochondria”). Mitochondria do not seem to form during early embryogenesis (Sathananthan and Trounson, 2000). They usually show an even distribution throughout the ooplasm in MI, MII, and early-fertilized oocytes. Significant changes in mitochondrial distribution and structure have been reported in pronuclear embryos, morulae and blastocysts (Motta et al., 2000).

\subsubsection{Lysosomes}

These organelles exist in a variety of forms, depending on their activity and stage of maturation. The primary lysosome is a small $(0.1 \mathrm{~lm})$ vesicular membrane bound organelle containing a dense core. Lysosomes are acid phosphate positive and probably arise from Golgi membranes (Sathananthan et al., 1993). They are sparse in mature oocytes but increase in numbers in ageing oocytes and embryos. Secondary and tertiary lysosomes are commonly found in GV oocytes and present bizarre images, a sign of atresia. Occasionally lysosomes may be found associated with dense phagosomes or located in multivesicular bodies. Multivesicular bodies show myriads of minute vesicles similar to pinocytotic vesicles found at the surface, while lipofuscin bodies are probably advanced lysosomes containing lipid inclusions. These increase with ageing in culture, a feature of ageing somatic cells.

\subsubsection{Changes in mRNA and Protein patterns}

Oocyte is heavily engaged to transcription during their growth and mRNA content of the oocyte changes during maturation. This switch in mRNA populations is brought about by both general termination of transcription at GVBD, and degradation of the select 
subset of transcripts. Generally, oocyte maturation does not require transcription except sheep where transcription is necessary (Moor and Crosby, 1986). In most cases, mature eggs are not transcriptionally active, as they are arrested in the metaphase of the second meiotic division. The sea urchin egg, however, is an exception, in that it is arrested before fertilization with a haploid pro-nucleus, which is transcriptionally active and selectively accumulates histone mRNA before fertilization (Venezky et al., 1981).

Protein synthesis patterns change significantly during the transition from an oocyte to a mature egg. Certain mRNAs are translationally activated while others become repressed (reviewed in Hake and Richter, 1997). Translational repression results in part from the degradation of mRNAs, but also from selective mRNA deadenylation. In sea urchins for example, production of yolk (Wessel et al., 2000b) and cortical granule content (Laidlaw and Wessel, 1994) proteins is extremely active in the primary oocyte, but ceases at the beginning of oocyte maturation, due to mRNA degradation. In Xenopus and mouse, a specific class of maternal mRNAs is deadenylated and translationally repressed during oocyte maturation (Paynton and Bachvarova, 1994). Translational activation of select mRNAs during oocyte maturation is achieved by regulated elongation of their poly (A) tail (Hake and Richter, 1997), and normally leads to a several-fold increase in the rate of overall protein synthesis (Wasserman et al., 1986). Translational regulation of Cyclin B has been studied during maturation constantly. The level of translational activity might vary in different species depends on their meiotic stage.

\subsection{Hormonal induction of oocyte maturation}

The hormonal signaling induces the oocyte maturation. Ovarian follicles synthesize the two major steroidal mediators, estradiol-17 $\beta$ and $17 \alpha, 20 \beta$-dihydroxy-4-pregnen-3-one $(17 \alpha, 20 \beta-D P)$ during oocyte growth and final maturation in response to FSH and LH, respectively. Unlike estradiol-17 $\beta$ (genomic action), 17 $\alpha, 20 \beta$-DP binds to a novel, Gprotein-coupled membrane receptor (non-genomic action) (Fig. 4), leading to the de novo synthesis of cyclin B, the regulatory component of maturation-promoting factor (MPF), which activates a preexisting cdc2 kinase via phosphorylation of its threonine 161 by a threonine kinase, thus producing the $34 \mathrm{kDa}$ active cdc2. Upon egg activation, MPF is inactivated by proteosomal-mediated degradation of cyclin B. 
An endocrine-disrupting chemical, diethylstilbestrol (DES), a nonsteroidal estrogen, triggers oocyte maturation in fish. The morphology (the time course of the change in germinal vesicle breakdown) and an intracellular molecular event (the de novo synthesis of cyclin B) induced by DES were indistinguishable from those induced by $17 \alpha, 20 \beta$-DP. A synergistic action of DES on $17 \alpha, 20 \beta$-DP-induced oocyte maturation was observed. An antibody against the $17 \alpha, 20 \beta$-DP receptor, inhibited both $17 \alpha, 20 \beta$-DP- and DES-induced oocyte maturation. These results suggest that DES may act on the $17 \alpha, 20 \beta$-DP receptor as an agonist of $17 \alpha, 20 \beta$-DP (Fig. 4).

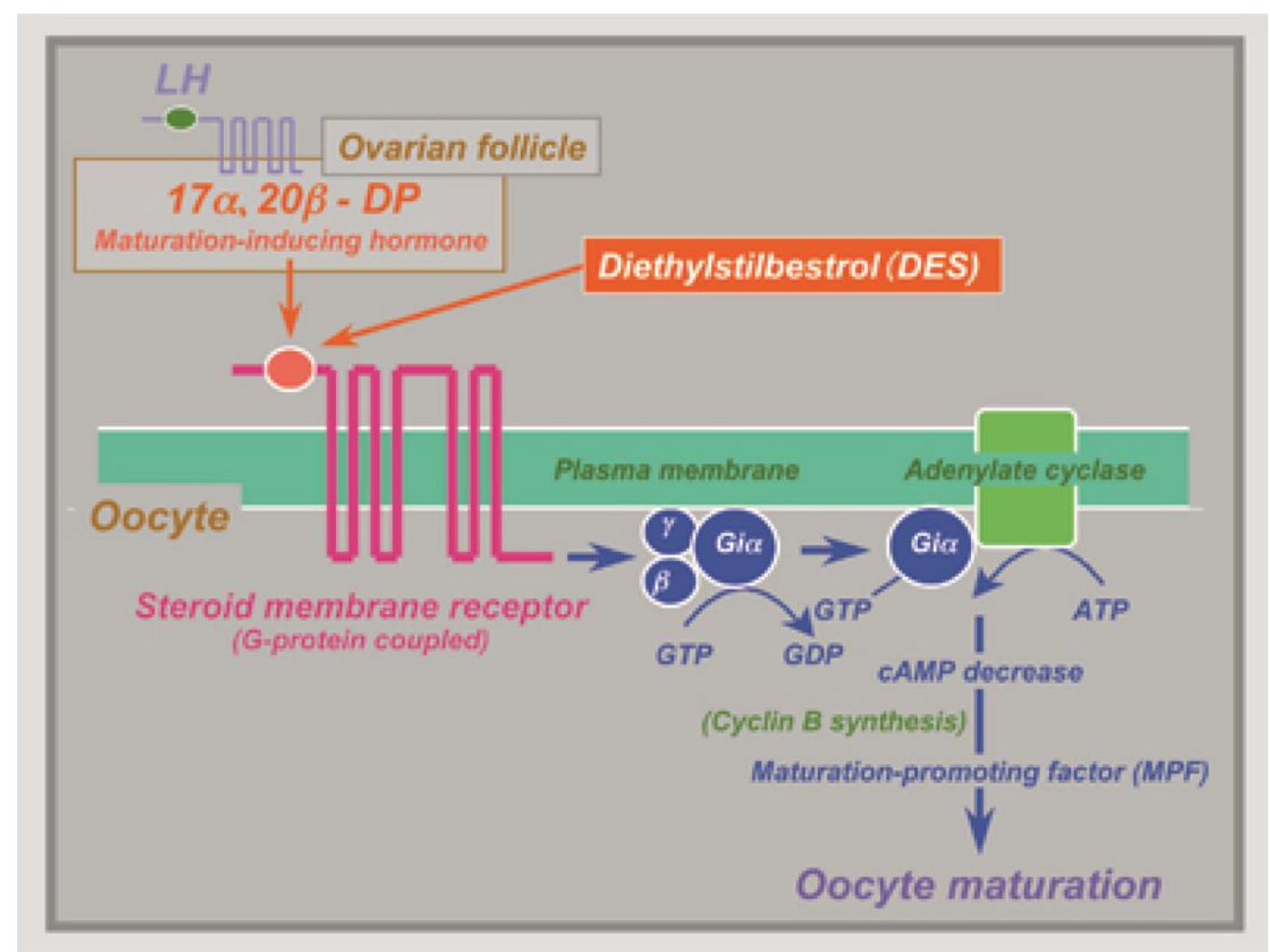

Figure 4. Hormonal regulation of oocyte maturation.

The sterol binds to the G-protein receptor, which activates the MPF (cdc2 and cyclinB) activation leads to reduction in cAMP and oocyte maturation. After egg activation, the cyclin B is degraded to keep the MII arrest.

\subsection{Essential developmental events during oogenesis.}

\subsubsection{Oocyte polarity and germ plasm assembly}

Oocyte being the base for the developing embryo considering the required changes in nuclear and cytoplasmic changes. The oocyte also involves in deciding some of the early 
necessary events like setting polarity, early axis specification and forming germplasm (Bontems et al., 2009; Marlow et al., 2008) which are crucial for proper embryonic development. Oocyte animal-vegetal polarity and dorsal-ventral axis is determined by localization of group maternal mRNA molecules and by balbiani body (Yvette G. Langdon and Mary C. Mullins, 2011). Germ cells are the immortal cells, which give rise to the next generation unlike somatic cells, and they are pre-formed in the oocytes by localizing group of mRNA, proteins and cellular organelles like mitochondria. During embryonic cleavage, they are taken up by few cells that will develop as germcells. Also germplasm organizer protein Oskar regulates rab11 polarization in Drosophila and trafficking in oocyte (Gretchen Dollar.2002). Hence, the transport pathway within the oocyte cell plays crucial role in positioning molecules in the proper place and separating every cellular compartment physically but connected through other processes.

\subsubsection{Accumulation of maternal factors}

The reduced DNA content and other cytoplasmic changes needs to store the necessary factors to regulate early development and nutrients to feed the developing embryo in oviparous animals while in the mammals the developing embryo is fed through the placenta. The embryo is dependent on stored maternal mRNA till at least the maternalzygotic transition, when transcription of embryonic genes begins in earnest, with a major burst of transcription occurring after mid blastula transition. The nutrients are taken up by the oocyte from the blood flow through receptor-mediated endocytosis or bulk endocytosis. So endocytosis is crucial for accumulation yolk protein in the oocyte.

\subsubsection{Vitellogenesis}

Vitellogenesis is a process of vitellogenin synthesis and deposition in the oocyte. The central nervous system induces secretion of gonadotropin-releasing hormone (GnRH) by specialized neurons. The GnRH acts on pituitary cells, which secrete the folliclestimulating hormone (FSH) which leads to the synthesis of sexual steroid hormones, such as $17 \beta$-estradiol. In response to the sterol signals, specific receptors in hepatocytes mediate the synthesis and release them into the blood of vitellogenins (Vtgs), the main yolk precursors in plasma (Fig.5). These are specifically incorporated by the oocytes via receptor-mediated endocytosis. (Stifani et al., 1990; Hiramatsu et al., 2004) These yolk proteins are stored in the inactive lysosome until they are required, then the yolk proteins are cleaved enzymatically once the oocyte matured for ovulation. Thus, cellular trafficking is necessary during oogenesis and early embryogenesis. 


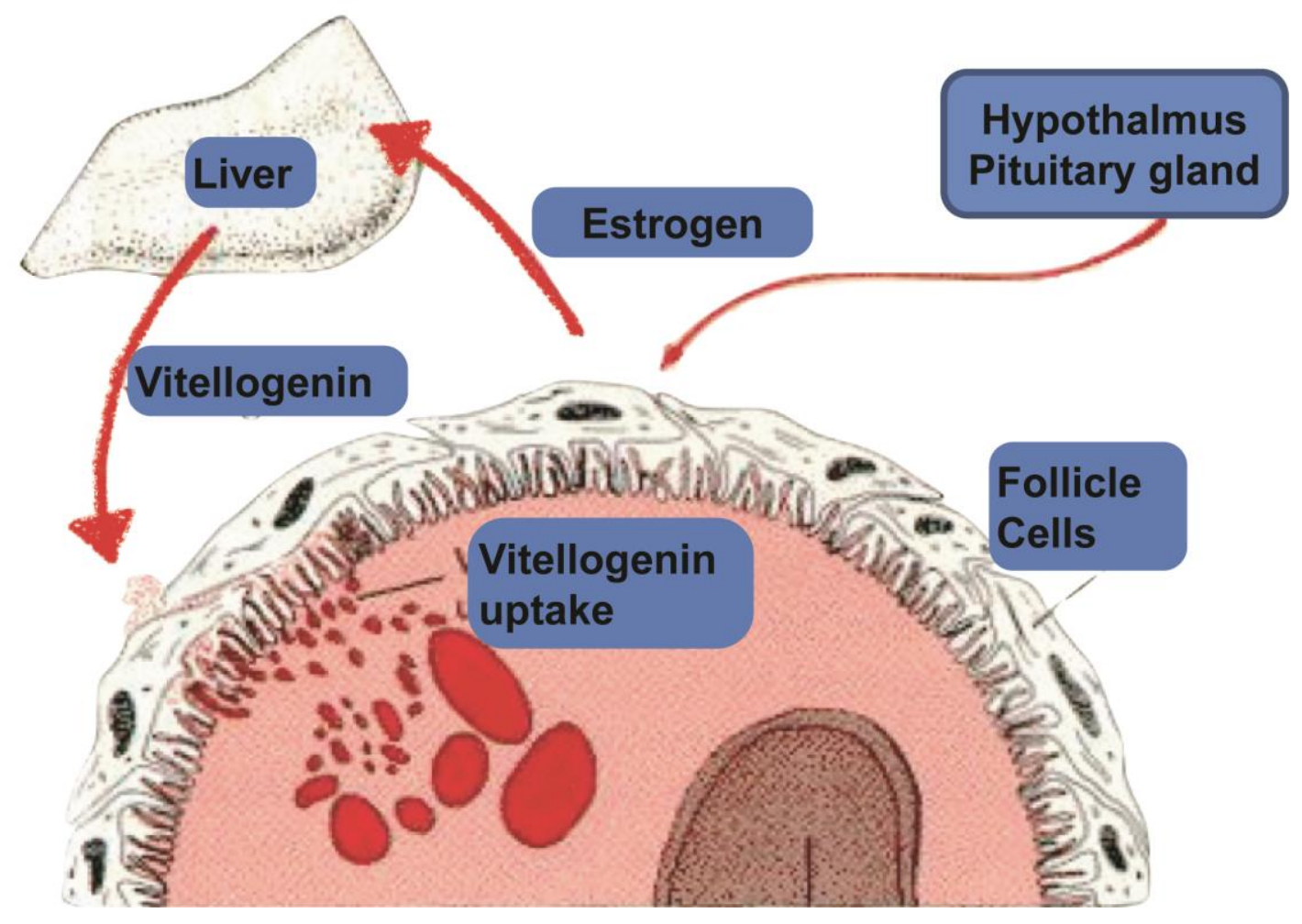

Figure 5. Vitellogenesis.

The cartoon shows synthesis of the yolk protein precursor in liver upon estrogen signal from the follicle cells. The vitellogenins are delivered in the blood flow and taken up by the oocyte through endocytosis. Yolk globule in red shows the lysosome with yolk.

\subsection{Vesicle trafficking during oogenesis}

Receptor-mediated endocytosis is a critical process utilized by oocytes of nonmammalian species, such as insects and frogs, to import yolk protein precursors (Opresko \& Wiley 1987). Import of vitellogenin occurs during oocyte growth through clathrinmediated endocytosis following binding to a receptor. Although mammalian oocytes do not produce yolk, it is possible that endocytosis could be important during oocyte growth. In addition, endocytosis of cell surface receptors could be important for the regulation of meiosis. Indeed, receptor trafficking is critical for the regulation of meiotic maturation in oocytes of Caenorhabditis elegans (Cheng et al. 2008). Thus, endocytosis is crucial for oocyte growth and storing essential components. Exocytosis is opposite to endocytosis by the process. Exocytosis plays important role after fertilization in avoiding polyspermy by the event of chorion elevation or cortical reaction. 


\subsubsection{Endocytosis}

Endocytosis is a vital process for every cells to intake molecules from outside and interact with the environment. Endocytosis plays a crucial role in many cellular events during developmental processes from oogenesis until the aging adult. Endocytosis is activated once the ligand binds to its receptor on the plasma membrane and then they are internalized into a vesicle mediated by clathrin. The clathrin-mediated vesicle matures into an early endosome regulated by Rab5 protein and then they are sorted into a multi vesicular body where different cargos are separated from their receptor and grouped based on their destination to lysosome or back to the plasma membrane. The multi vesicular body is regulated by Rab7 protein. Finally the lysosomal destined vesicles reach the lysosome by fusion while the plasma membrane targeted cargos are recycled back to the membrane through the recycling endosome regulated by Rab11 (Fig. 6). The lysosome is the main degrading organelle to clean cellular debris by enzymatic cleavage (Gary J. Doherty and Harvey T. McMahon, 2009). The lysosome is involved in many different processes like autophagy, mitochondrial degradation, and lipid transport to mitochondria etc.

Endocytic membrane trafficking involves the cellular internalization and sorting of extracellular molecules, plasma membrane proteins and lipids. Endocytosis is required for a vast number of functions, including nutrient uptake, cell adhesion and migration, receptor signaling, pathogen entry and cell polarity. It is well established that endocytosis regulates receptor-mediated signaling. In single cell organisms, the process of endocytosis has been studied and discovered many novel regulators like rab proteins. However, a genetic screen in C.elegans a multicellular organism discovered novel regulators and novel cellular compartment suggesting that higher organisms and vertebrates need a sophisticated, tighter level of regulation than single cell organisms (Barth D. Grant \& Julie G. Donaldson, 2009).

\section{Figure 6. Endocytosis.}

Endocytic vesicles that are formed by invagination and pinching of clathrin-coated pits become uncoated in the cytoplasm and fuse with specialized membrane organelles known as endosomes, from which receptors and their ligands are sorted to various intracellular destinations. The cargos go through early, late endosome to reach lysosome while internalized molecules can be recycled back from early endosomes or a late recycling compartment to the plasma membrane and, therefore, participate in several rounds of endocytosis. Endocytosed receptors can also be sequestered in endosomes for a long time or transported to late endosomes and lysosomes, in which they are proteolysed. Late endosomes are referred to as multivesicular bodies (MVBs) because of 
their intra luminal vesicle. Lysosomal enzymes and other molecules are delivered from the Golgi apparatus to endosomes and plasma membrane by means of clathrin-mediated or other types of membrane transport (Sorkin and Von Zastrow.2002).

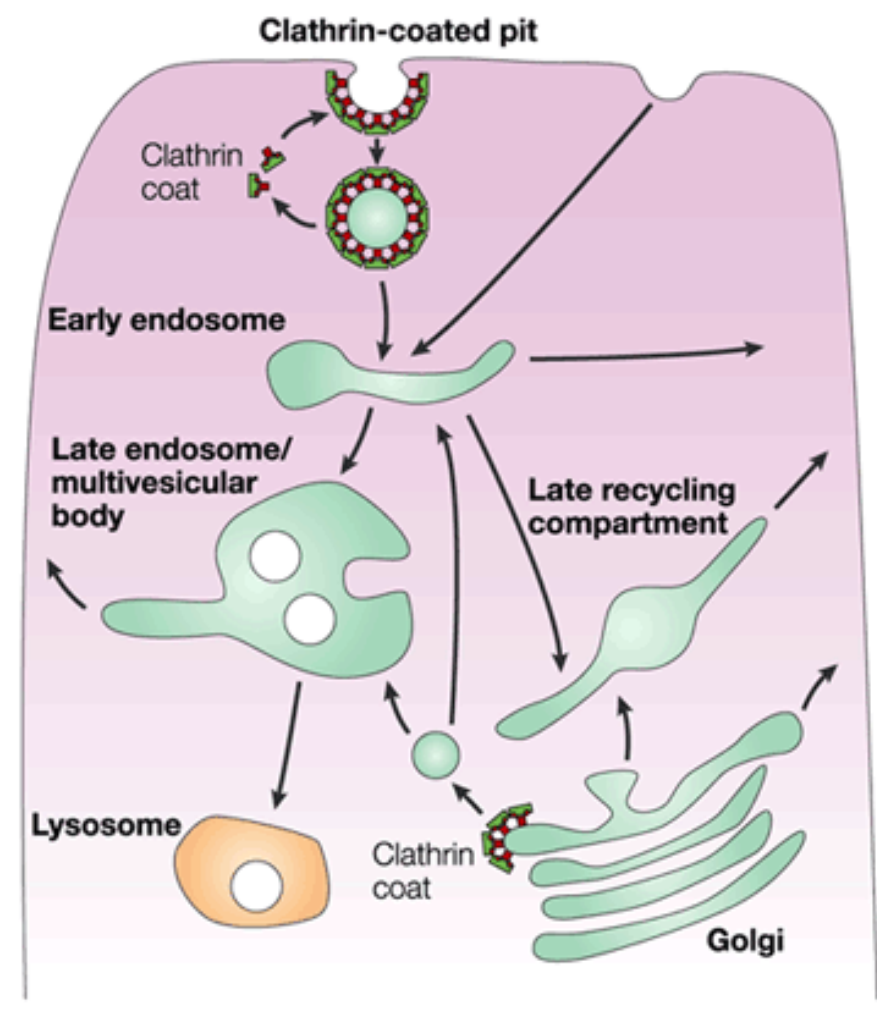

Nature Reviews | Molecular Cell Biology

\subsubsection{Exocytosis}

Exocytosis is an opposite process to endocytosis by which the cell directs the secretory vesicle content out of the cell by fusing with the plasma membrane. There are two types of exocytosis. 1. Constitutive exocytosis- which is delivering the content irrespective of any outside signal like cell wall proteins or extra cellular matrices.2. Regulated exocytosis- process needs an external signal to induce the vesicle fusion in specialized cell types like neuron or oocytes (Fig.7). This is a five-step process. Vesicle transport, tethering, docking to the membrane, vesicle priming and fusion. Lot of study has been done to understand the process of exocytosis and fusion in neuron in vivo and in vitro. Many SNARE proteins have been discovered to play a role in fusion during exocytosis (Reinhard Jahn \& Dirk Fasshauer). The process is involved in many biological processes such as turnover of plasma membrane, secretion of enzymes, neurotransmitter release, acrosome reaction during fertilization and chorion elevation / cortical reaction in human during fertilization. During oogenesis a type of secretory granule are produced by the Golgi 
and stored until the time of fertilization. Immediately after fertilization, they are activated to fuse with the plasma membrane and deliver their cargos, which elevate the chorion membrane and modify the zona pellucida to avoid polyspermy that block the entry of more sperm into the oocyte, which is detrimental to the embryo. Hence, both endocytosis and exocytosis plays essential role during oogenesis and fertilization.

\section{Figure 7. Exocytosis.}

Many soluble proteins are continually secreted from the cell by the constitutive secretory pathway, which operates in all cells. This pathway also constantly supplies the plasma membrane with newly synthesized lipids and proteins. Specialized secretory cells have, in addition, a regulated exocytosis pathway, by which selected proteins in the Trans Golgi network are diverted into secretory vesicles, where the proteins are concentrated and stored until an extracellular signal stimulates their secretion. It is unclear how aggregates of secretory proteins are segregated into secretory vesicles. Secretory vesicles have unique proteins in their membranes; perhaps some of these proteins act as receptors for secretory protein aggregates in the Trans Golgi network (Essential cell biology, 2004, Garlend Science).

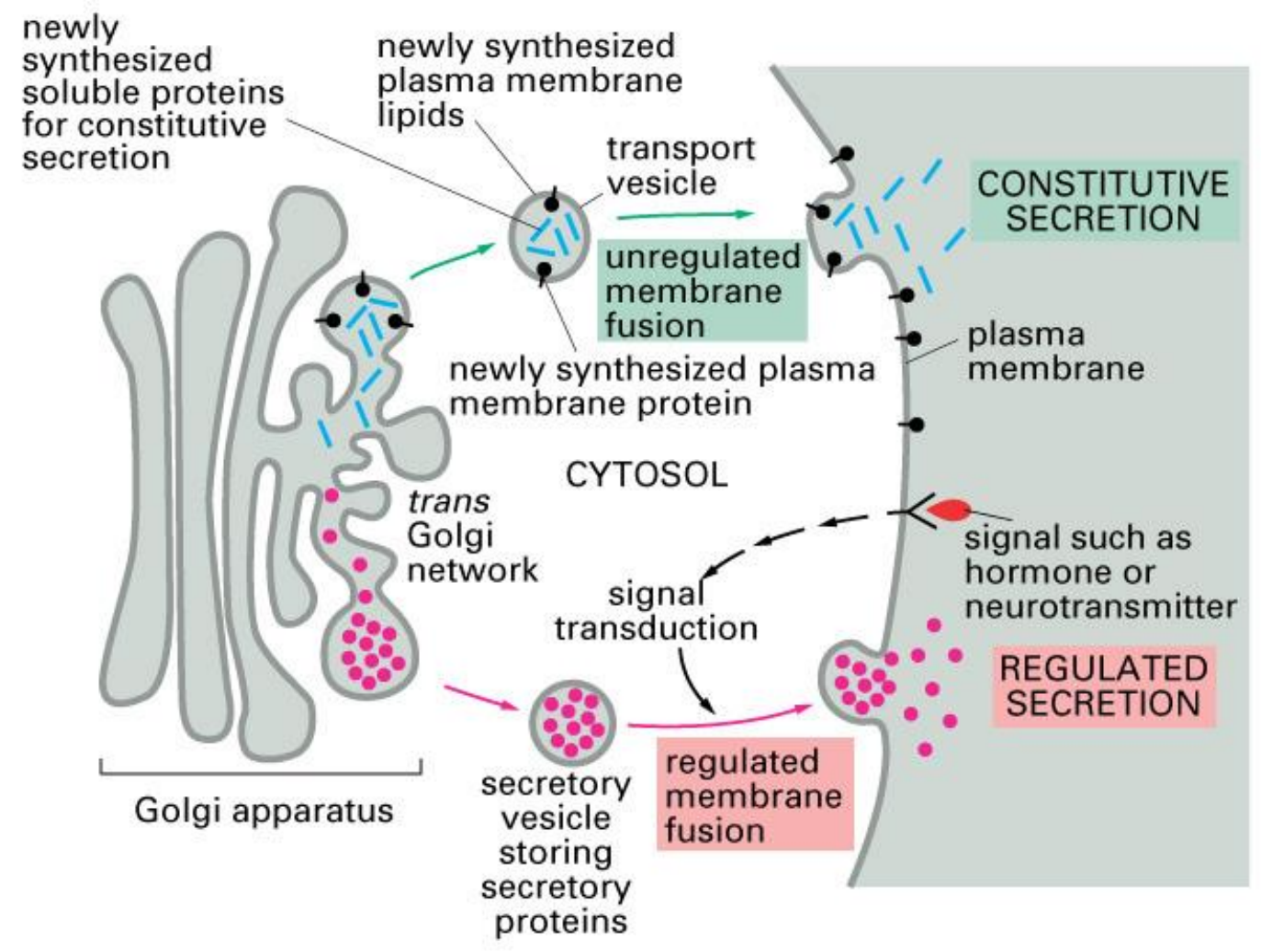




\subsection{Investigating the event of yolk endocytosis in invertebrates}

The yolk protein endocytosis has been mostly studied in-vivo in C.elegans oocytes. The yolk proteins of C. elegans are lipid transport proteins homologous to the ApoB-100 component of mammalian low-density lipoprotein complexes (LDL). Yolk in worms is a lipoprotein complex composed of yolk proteins, cholesterol, phospholipids, and triglycerides. Yolk storage vesicles in oocytes are considered to be functional analog of lysosomes, except that they lack high-level hydrolytic activity during oocyte growth, as the embryos mature, some yolk granules acquire hydrolytic activity and digest their contents. In Xenopus oocytes, yolk granules stochastically acquire a reduced lumenal $\mathrm{pH}$, which presumably activates acidic hydrolases. In C. elegans, yolk proteins bind directly to cholesterol, as demonstrated by cross-linking studies with photo-activatable cholesterol analogs. Animals with mutant oocytes that are unable to take up the yolk accumulate the yolk protein and cholesterol complexes in the pseudocoelom. Yolk granules of the oocyte and embryo contain little cholesterol; rather, internalized cholesterol appears to be distributed among intracellular membranes. Presumably, soon after uptake into the endosomal system, the cholesterol is stripped from the yolk particles and transported throughout the cell. Yolk transport particles and their cognate receptors in oocytes probably represent the evolutionary precursors of the LDL transport and uptake systems of mammals.

At a specific time-point in C. elegans embryogenesis, all of the remaining yolk particles are resecreted into the perivitelline space, quickly followed by their accumulation in endocytic vesicles of the embryonic intestine. After hatching, a significant proportion of the yolk remains in the intestine, where it presumably provides a nutrient source for foraging larvae. A number of endocytosis assays have been developed to study the mechanisms of endocytosis in C.elegans. One of these follows the trafficking of a chimeric yolk protein (yolk protein fused to green fluorescent protein) (YP170::GFP) in transgenic strains. YP170::GFP synthesis, secretion, and endocytosis recapitulate the transport steps taken by endogenous yolk as judged by immunofluorescence and immune-EM studies; thus, YP170::GFP represents an excellent tool for assaying secretion and endocytic trafficking in a live metazoan animal. The YP170::GFP reporter has been used in reverse genetic studies in $\mathrm{C}$. elegans to probe the function of known or candidate endocytosis factors identified in the complete genome sequence of $\mathrm{C}$. elegans. Genetic screens for mutants defective in receptor-mediated endocytosis and trafficking using the YP170::GFP assay yielded 12 rme genes (rme - receptor mediated endocytosis). Extensive analysis has 
been performed on a yolk uptake-specific mutant rme-2, as well as two mutants with widespread defects in endocytic trafficking, rme-1 and rme-8. rme-2 encodes a member of the LDLR family of lipoprotein receptors, while rme-1 and rme-8 encode new cytosolic factors associated with endosomes. rme-2 mutants lack any detectable yolk in their oocytes and embryos and have severely reduced brood sizes. Several lines of evidence indicate that the RME-2 receptor is both necessary and sufficient for yolk endocytosis in C. elegans and therefore is the sole yolk receptor of C. elegans. RME-2 expression is restricted to oocytes. RME-2 colocalizes with its putative ligand YP170::GFP within putative sorting endosomes when an intermediate step in endocytic trafficking is blocked. Furthermore, forced expression of RME-2 in ectopic cell types is sufficient to induce YP170::GFP accumulation in those cells. First, immunoelectron microscopy revealed that most RME-2 is found in subsurface vesicles and not in pit structures or plasma membrane clusters. Second, functional studies that blocked the early steps in endocytosis caused RME-2 to accumulate on the plasma membrane in a diffused pattern and showed a strongly reduced accumulation in these subsurface vesicles, indicating that they are derived thruogh endocytosis and not by secretion. Finally, RME-2-positive vesicles do not accumulate the ligand (yolk). Thus, in the oocyte, RME-2 represents an important marker for recycling endosomes, indicating necessity of high-level regulation in multicellular organisms (Hanna fares and Barth Grant, 2001).

\subsection{Discovering novel regulators of oogenesis in vertebrate}

The oocyte is a special cell type in which complex and dynamic cellular events take place during oogenesis to prepare the oocyte for embryogenesis. The process is tightly regulated. Many important events like polarity, embryonic axis and germplasm specification take place during oogenesis and more efforts have been put to find and understand the process in invertebrates like C.elegans and Drosophila.The genetic screen in C.elegans found novel regulators and compartment for early recycling compartment (ERC), which led to the belief of novel regulators in vertebrates. (Grant and Hirsh.1999; Shaw et al.,2001; Balklava et al.,2007) beyond the yeast studies (Grant and Donaldson, 2009). So, our lab was interested to find novel regulators during oogenesis in vertebrates using maternal effect genetic screen in zebrafish as a vertebrate model.

\subsection{Zebrafish-vertebrate model}

Zebrafish has been used as a model organism in developmental biology since the 1950 and has become increasingly popular in research during the last 30 years to study 
cellular, molecular, and genetic interactions (Streisinger et al.,1981). This success is related to several attributes of the zebrafish. Zebrafish is easy to maintain and mature females are able to lay several hundred eggs per week. In contrast to other vertebrate model organism, embryos develop rapidly and the generation intervals take three to four months compared to the mouse. Furthermore, zebrafish embryos are transparent and simple organized. Based on the optical clarity and the large size, the developing embryo can be observed using standard dissecting microscopes. 12 hours post fertilization (hpf), the body axis and the overall body plan is apparent and after $24 \mathrm{hpf}$ all the major organ primordia are formed (Kimmel et al.,1995). Since mutations are easy to induce, large-scale screens have been carried out to identify mutations causing defects in particular biological processes. Moreover, embryological techniques used in Xenopus can be applied in the zebrafish including transplantation experiments, microinjections of sense mRNA for overexpression studies or of antisense oligo-nucleotides (morpholinos) for knockdown analysis also economically feasible.

\subsection{Zebrafish oogenesis}

During oogenesis, the oocyte passes through four different stages of differentiation: The primary growth stage (Stage IA+IB), the cortical alveolus stage (Stage II), the vitellogenesis stage (Stage III), the oocyte maturation (Stage IV), and the ovulated, mature egg (Stage V).

\section{Figure 8. Zebrafish oogenesis.}

This picture shows the stages of zebrafish oogenesis. They are arranged in a progressive order, which is not the case in the ovary. The drawing is not scaled to the size. a. the germcells. b. In stage $1 \mathrm{~B}$, notice the germplasm components in red localized just below the nucleus. c. In stage II the the animal pole markers localized in green. The transparent circular vesicles are cortical granules or cortical aveoli. d. Dark grey vesicles are the yolk-filled lysosome, which creates the opaqueness in stage III. Notice the nucleus in the middle in stage I to stage III. e. and f. Stage IV and V is transparent after maturaion and also the germplasm components moved to the cortex and ready to fertilize. g. The fertilized embryo where the cytoplasm is at the top in green with animal pole components and red arrows show the transport (Selman et al., 1993). 


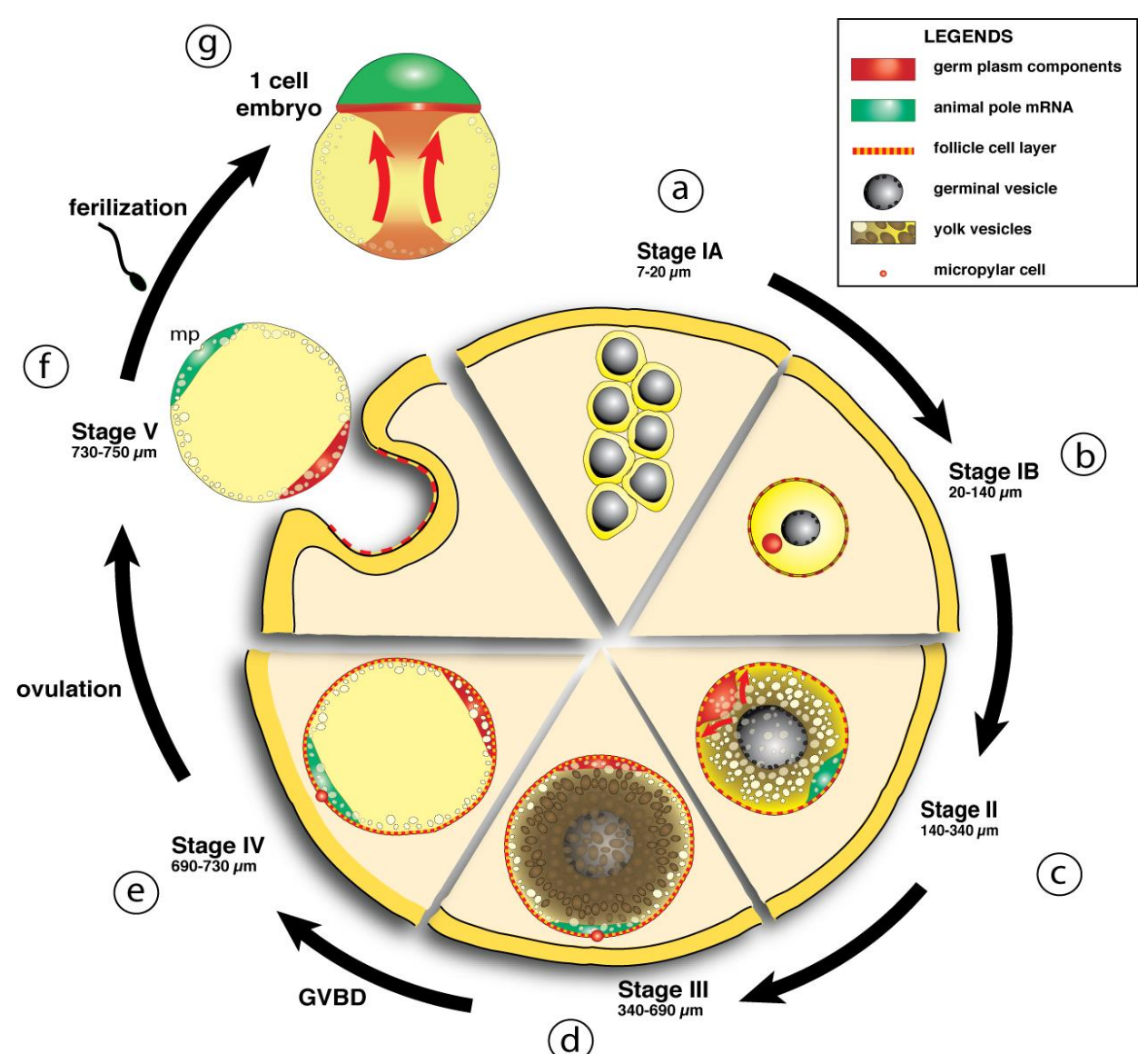

During the prefollicle stage of the primary growth phase (stage IA, oocyte diameter $7-20 \mu \mathrm{m})$ the oocyte undergoes chromosome condensation reflecting the entry into the first meiosis. At the same time, numerous nucleoli begin to accumulate in both the periphery and inner membrane of the nucleus. During the follicle stage (Stage IB, follicle diameter 20-140 $\mu \mathrm{m}$ ) chromosomes decondense and the oocyte increases the transcription of maternal genes. The follicle cells and the oocyte extend microvilli to contact each other and components of the vitelline envelope accumulate between the follicle cells and the oocyte. Subcellular structures like endoplasmatic reticulum, Golgi, and mitochondria become abundant and the Balbiani body develops adjacent to the nuclear envelope. The second stage (follicle diameter 140-340 $\mu \mathrm{m}$ ) is characterized by the formation of bound vesicles, the cortical alveoli. During egg activation, these vesicles will become anchored at the cortex and undergo exocytosis to modify the vitelline membrane. During the vitellogenesis stage (Stage III, follicle diameter 340-690 $\mu \mathrm{m}$ ) the oocyte acquires large amounts of vitellogenin by endocytosis, which serve as an energy source for the developing embryo. The oocyte maturation (Stage IV, follicle diameter 690-730 $\mu \mathrm{m}$ ) is characterized by the movement of the nucleus towards the animal pole of the egg, the breakdown of the nuclear envelope, and the arrest of meiosis at the second meiotic metaphase. At this stage, the 
oocyte enlarges about $10-15 \%$ probably by the proteolytic cleavage of yolk proteins and the endocytosis has finished. The final stage (Stage V, egg diameter 730-750 $\mu \mathrm{m}$ ) involves the release of the oocyte from the oocyte/follicle complex into the lumen of the ovary (Fig.8) (Nazan Deniz Koç et al., 2008).

A fish oocyte increases its volume a few hundred fold within 10 days. This massive growth is based on the uptake of yolk proteins that represents the nourishment store for the developing embryo (Pelegri.2003). The primary component of the yolk proteins, vitellogenin, is exogenously synthesized by the liver of the mother and is internalized into the oocyte by clathrin-dependent endocytosis, then enzymatically cleaved and stored (reviewed in Romano et al.,2004). Thus, the oocyte is probably the most active cell in the female zebrafish for endocytosis and hence an excellent model to study its regulation in a vertebrate.

\subsection{Genetic screen in zebrafish oocyte}

In contrast to the limitations of transient depletion, mutants embryos with genetic lesions caused by chemical mutagens, radiation, retroviral insertion, or zinc finger nucleases disrupting genes required maternally for development of oocytes or early embryos allow perturbation of gene function at the earliest stage when it is required without causing off-target effects. Such maternal-effect mutants have been discovered in large-scale mutagenesis screens in model organisms including Drosophila melanogaster (flies), C. elegans (worms), Danio rerio (fish) and made by homologous recombination to disrupt candidate genes in Mus musculus (mouse) (Pelegri , 2003 \& Pelegri and Mullins, 2004). Though maternal-effect screens have only recently been carried out in vertebrates, such mutants have already identified novel maternal-effect functions for genes with essential maternal roles during early embryonic development. The maternal-effect screens in zebrafish yielded the largest collection of maternal-effect mutants in vertebrates; nevertheless, only a fraction of the total expected were identified, based on the number of genomes screened and estimates from previous large-scale zygotic screens (Mullins et al., 1994; Solnica-Krezel et al., 1994; Driever et al., 1996). The zebrafish maternal-effect screens combined to date approach these numbers; however, these screens select against maternal genes that also have essential zygotic functions (Dosch et al., 2004; Pelegri et al., 2004). The genetic screen for maternal effect mutants in zebrafish oocyte produced different group of mutants having a defect during oogenesis and early embryogenesis 
before mid-blastula transition (MBT) (Dosch et al., 2004). I am interested in one group called opaque egg mutants.

\subsection{Opaque egg mutants}

These mutants lay opaque egg instead of their normal transparent eggs in wildtype. Also they don't segregate their cytoplasm from the yolk towards animal pole. These mutants show this symptom irrespective of the male parent genotype, thus confirming the presence of mutated maternal gene. This group consisted of four mutants with similar phenotype, which were mapped to four different chromosomes, so they are four different genes, which suggests that they could work in a same biological process since they produce similar phenotype (Fig.9).

The opaque phenotypes suggest that these mutant having a defect in endolysosomal degradation of yolk proteins in the oocyte lysosome. During the oocyte maturation from stage III opaque oocyte to stage IV transparent oocyte, the stored yolk protein in lysosomes are cleaved enzymatically by a group of cathepsin and cystein proteases which changes their opaqueness to transparent. This indicates that opaque egg mutants are blocked before the enzymatic degradation takes place. Hence, the protein profile of stage V mutant oocytes were compared to premature stage III and mature stage V wild-type oocytes using Coomassie-stained SDS-gels (Fig. 10). The mutant oocytes are characterized by a protein pattern similar to stage III immature wild-type oocytes that suggests; in the mutants, the enzymatic degradation has not occured. This suggested that these mutants have a role in endosomal trafficking and yolk degradation.

\section{Figure 9. Opaque eggs mutant phenotype.}

(A) Live, wild-type embryo 30 minutes post fertilization (mpf). (D) Stage IV wild-type oocyte. (B, C, E, F) Opaque egg mutant's ruehrei, over easy, soufflé and sunny side up at $30 \mathrm{mpf}$ in incident light. Oocytes from mutant females are more similar to immature oocytes (changed after Dosch et al, 2004). 

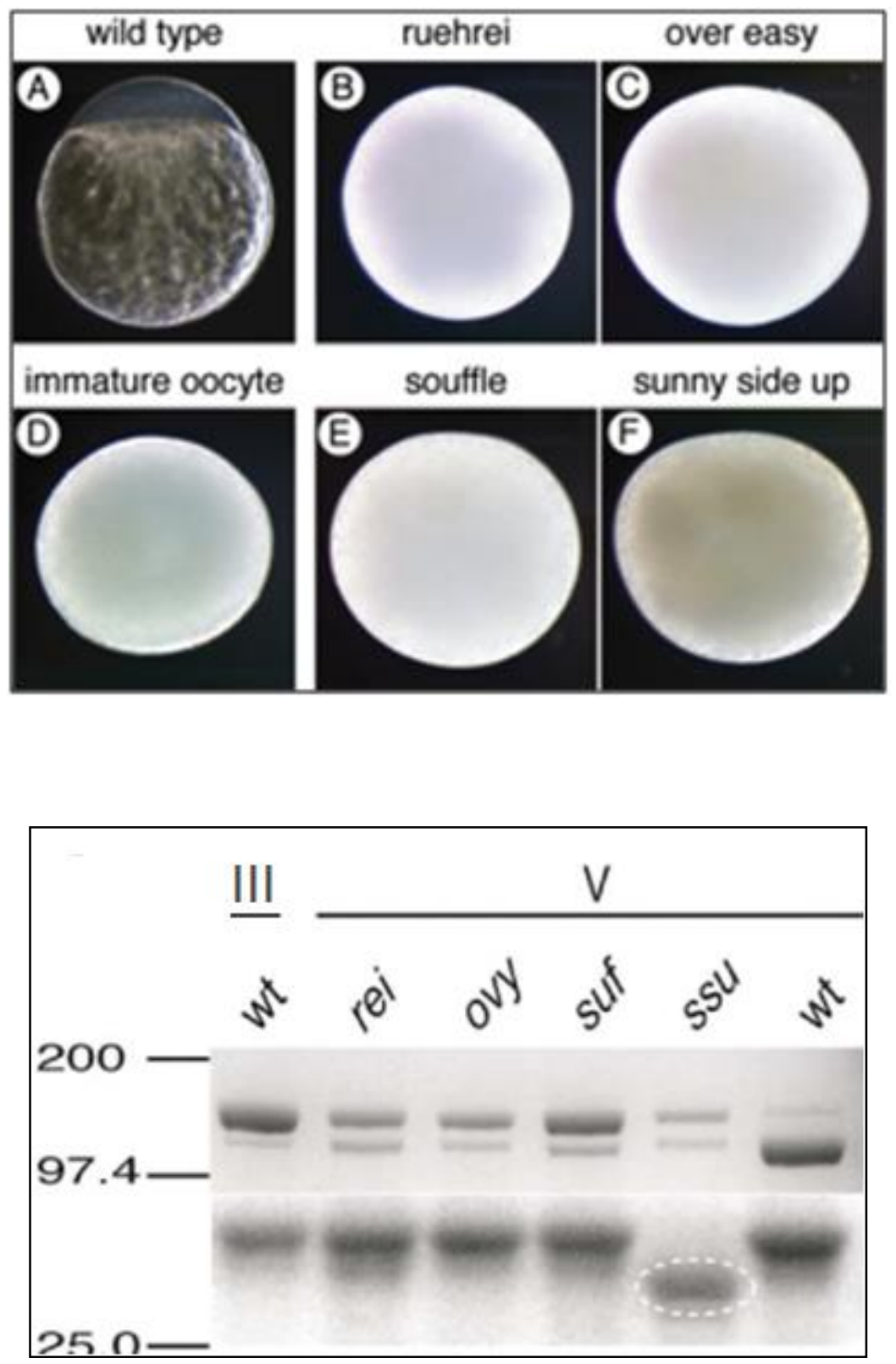

Figure 10. Yolk protein profile of opaque egg mutants.

Coomassie-stained SDS page of yolk proteins of stage III and V wild-type (wt) eggs compared to stage V mutant eggs ruehrei (rei), over easy (ovy), soufflé (suf) and suny side up (ssu). Mutant eggs display a stronger band of the larger molecular-weight yolk protein, similar to stage III wt eggs. All other protein bands seem to be identical except of one protein band in sunny side up eggs (strippled oval) (changed after Dosch et al, 2004).

\subsection{Souffle encodes zebrafish homolog of SPASTIZIN}

Based on the strongest phenotype in all assays the suf mutant seems to be the most interesting candidate for further investigations. To characterize the molecular mechanism of the defect, we positionally cloned the disrupted gene in suf mutants. We previously located the suf mutation on chromosome 13 of the zebrafish genome. Genotyping 1183 
females identified 5 recombinants with the SSLP marker z25580/G47633 and another 5 recombinants with $\mathrm{z} 21403 / \mathrm{G} 41743$ restricting the critical interval with the suf mutation to 1.22 Mb (Fig. 11a). Generating novel SSLP markers AL13-10 and AL13-13, we reduced the interval to $270 \mathrm{~kb}$. Within this genomic region, we sequenced the cDNAs of arginase, vtilb (t-snare), rdh12 (retinoldehydrogenase 12), zfyve26 (spastizin), galectin and pleckstrin2 (Fig. 11b). The zfyve26 gene consists of 41 exons and encodes a predicted mRNA of 7798 bp (Fig. 11c). Comparing the zfyve26 cDNA-sequence between wild type and the p96re allele of suf mutants showed a 25 bp deletion at the 3'-end of exon 35 (Fig. 11d). However, the genomic sequence revealed a single point mutation in a splice donor, which probably results in cryptic splice donor selection 25 bp upstream of the wt splice site (Fig. 11d). The deletion of 25 bp results in a frame shift, which creates a termination codon after six aberrant amino acids (Fig. 11e). The premature STOP deletes 282 of the 2552 amino acids in the predicted Zfyve26 protein resulting in a shortened protein of 2270 amino acids (Fig. 11f). Searching for protein motifs with the Prosite database (Sigrist et al.,2010) and MyHits (Pagni et al.,2007) revealed a bipartite nuclear localization signal (amino acid 714-730), a serine rich domain (aa 1251-1342) and a zinc finger FYVE domain (aa 1807-1865). FYVE domains bind to the lipid phospatidylinositol-3-phosphate (PI3P) predominantly present on endosomes (Stenmark et al.,1996; Burd and Emr.1998; Gaullier et al.,1998; Patki et al.,1998). Phylogenetic analysis detected one homolog in most metazoan genomes and a similar gene in Drosophila melanogaster (CG5270), albeit no homolog in the C. elegans genome (Fig. 1Ig). Remarkably, although teleosts underwent an additional genome duplication compared to tetrapods (Postlethwait.2007), all ten teleosts genomes available at the Ensembl-database (http://www.ensembl.org) contain a single paralog of Suf/Spastizin, which we confirmed for zebrafish by BLAST searches with fulllength Suf. Alignment of the vertebrate protein sequences discovered a highly conserved Cterminus, which we termed Suf-domain. Scanning the zebrafish genome with the Sufdomain did not detect other proteins with a similar amino acid motif. This motif is also conserved in plants, but the protein does not contain a FYVE-domain (data not shown). In human tissue culture cells, this domain in SPASTIZIN interacts with BECLIN1, KIF13A and TTC19 (Sagona et al.,2010; Sagona et al.,2011) and is predicted to form alpha-helical solenoids as found in Clathrin heavy chain (Hirst et al.,2013a). Since the Suf domain is deleted in the p96re allele, it is essential for the Suf function during zebrafish oogenesis. Together, these data identified souffle as the zebrafish homolog of SPASTIZIN. 


\section{Introduction}

\section{Figure 11. Cloning of suf mutation.}

a. Genetic map of the suf-locus on chromosome 13. Meiotic mapping located the mutation between the markers z25580/G47633 ( $0.4 \mathrm{cM}$, centiMorgan; 5 recombinants/1183 females) and z21403/G41743 (0.4 cM) corresponding to a physical interval of $1.22 \mathrm{Mb}$ (megabases). Fine mapping of the suf-locus identified markers AL13-10 (0.08 cM) and AL13-13 (0.2 cM), which physically represents $280 \mathrm{kB}$. b. Among other genes, this interval contains the ArgII, vti1b, rdh12, galectin and pleckstrin2 genes, whose cDNA sequence did not display mutations in comparison to the database genome (http://www.ensembl.org/Danio rerio/Info/Index). c. Exon-intron structure of the suf gene. $\mathrm{d}$. The $p 96 r$ allele carries a G-A transition at the genomic level destroying a splice donor site in the transcribed RNA. e. The selection of an alternative, upstream splice donor leads to a deletion of 25 nucleotides causing a frame shift in the cDNA encoding six aberrant amino acids and eventually creating a premature STOP-codon (asterisk). f. The mutant protein is predicted to lack 282 amino acids at the C-terminus including the conserved SUF domain from amino acid 2375 to 2552. g. Phylogenetic diagram displaying the conservation of Souffle proteins among vertebrates. The Anopheles and Drosophila proteins were used to root the tree. Numbers indicate bootstrap-values and the scale the number of substitutions per amino acid residue. 
a

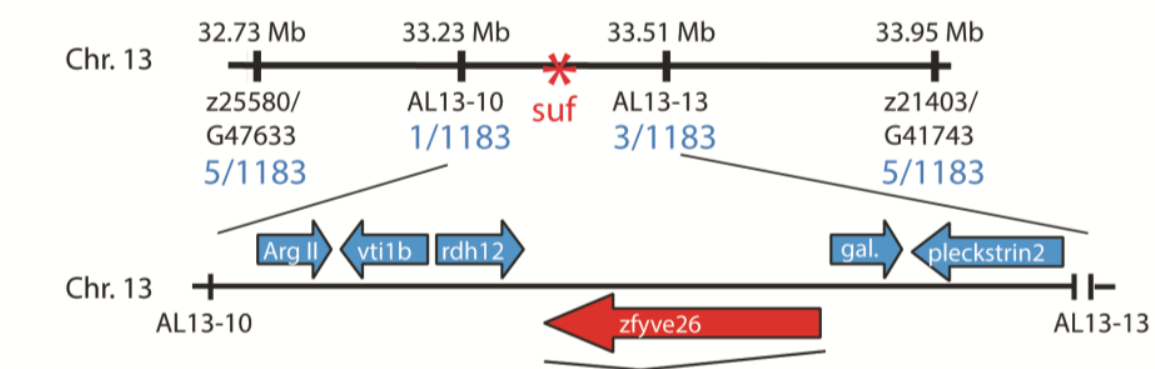

C

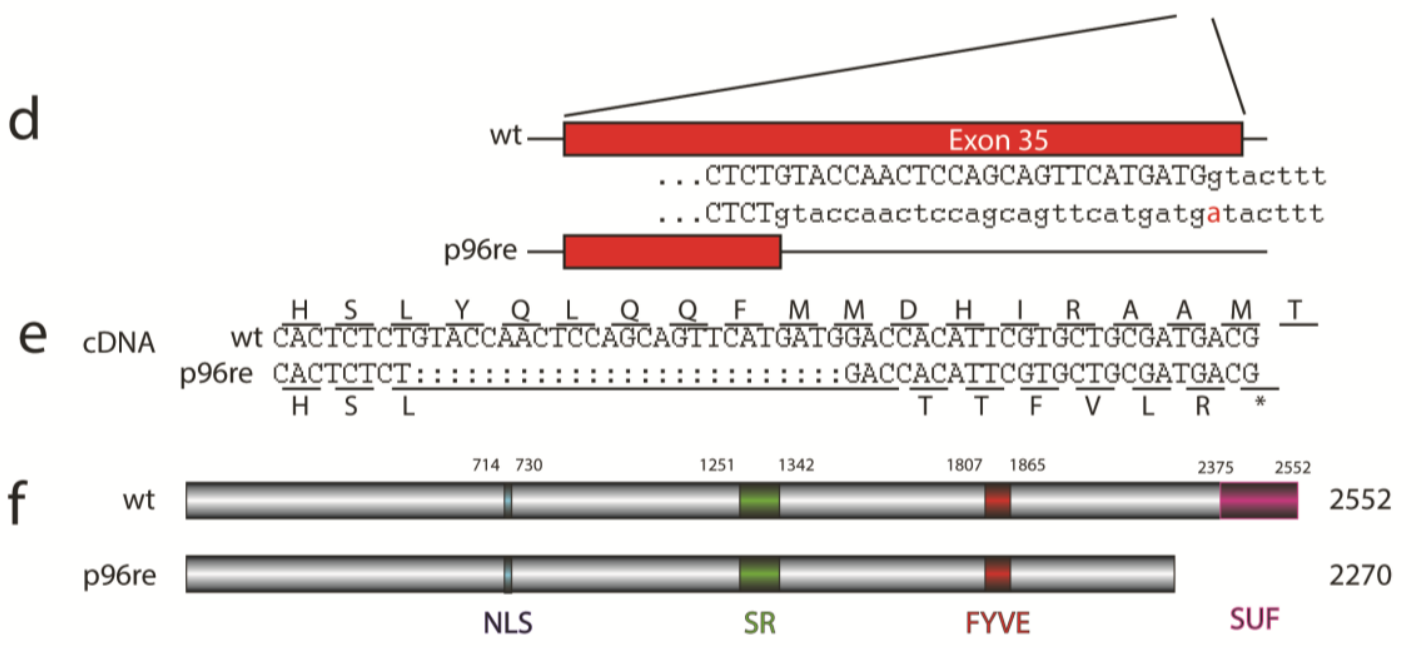

g

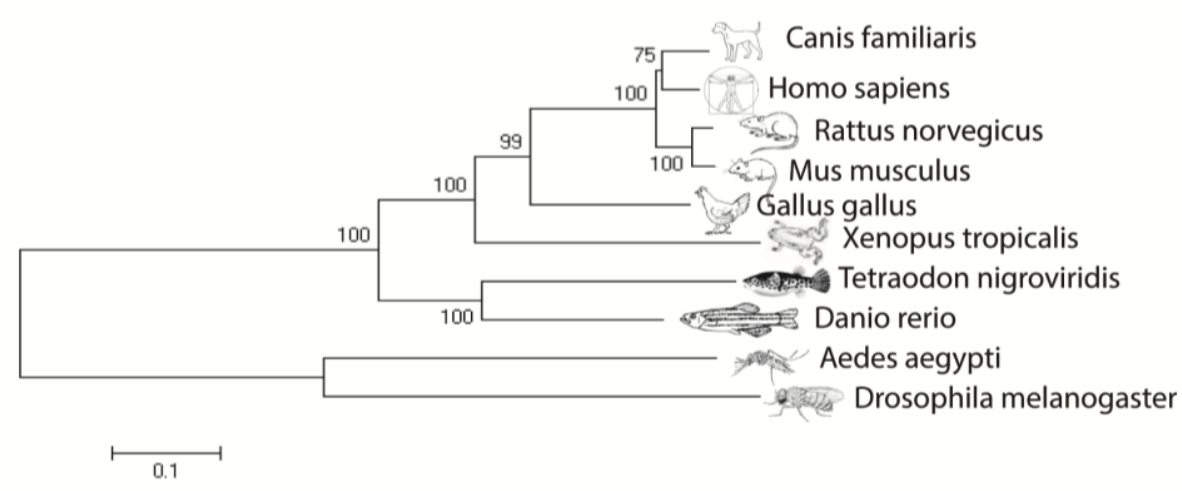

\subsection{Souffle is maternally expressed}

Although suf gene has been shown to express in different tissue during development, we see the mutant phenotype in oocyte. Hence, we investigated whether the Suf/Spastizin gene is expressed at the appropriate time to control oogenesis in zebrafish. Real-time PCR analysis of isolated follicles at selected stages showed that Suf/Spastizin mRNA is expressed during oogenesis with a slight increase at the onset of vitellogenesis (stage II) and another increase after ovulation (stage V) (Fig. 12a). This expression profile is consistent with a role during zebrafish oogenesis.

During embryogenesis, Suf/Spastizin mRNA decreased after 4 hpf (hours post fertilization) similar to other maternal genes (Fig. 12b). At $30 \mathrm{hpf}$ Suf/Spastizin showed a 
small peak of expression. Later during larval stages, the expression steadily increases consistent with microarray data in the Espresso database (http://zfespresso.tuebingen.mpg.de; Unigene ID: Dr.21642). To analyze sex-specific expression of suf/spastizin, we compared mRNA levels in whole females, females without ovaries and males. Contrary to its specific phenotype in the oocyte, Suf/Spastizin was strongly expressed in males and even outside the female germline suggesting that it also acts in somatic cells (Fig. 12c).
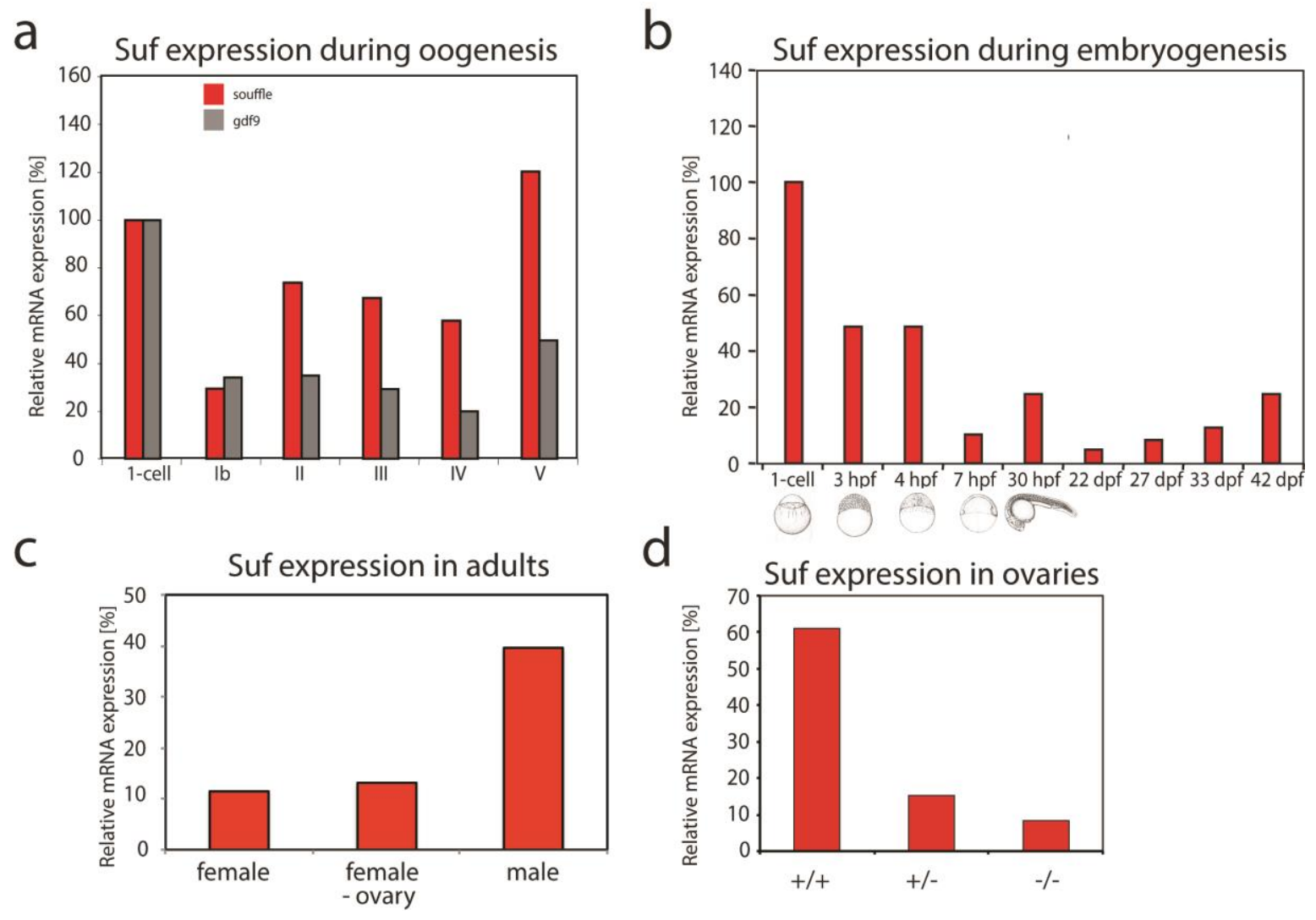

\section{Figure 12. Suf mRNA expression.}

The diagram shows the suf mRNA expression analysis in quantitative realtime PCR. a. suf mRNA level in percentage during oogenesis. Suf in red and gdf 9 in grey as a control. b. Suf expression during embryogenesis from 1 cell to $42 \mathrm{dpf}$. Dpf- Day post fertilization. c. Suf expression in adults. d. Suf expression in ovaries I different genotype. +-indicates the presence wt allele and - indicates the suf mutant allele.

The higher expression of Suf in males can be explained by two non-exclusive reasons. A simple explanation might be technical i.e. the genes gapdh and odc1, which we used to normalize mRNA levels, are differentially expressed between males and females as reported for efl $\alpha$ Groh et al. (2013). Alternatively, Suf is indeed higher expressed in males, 
possibly in one of the non-reproductive organs with sex-specific gene expression such as the brain or the liver (von Hofsten and Olsson.2005). However, the p96re allele clearly demonstrates that Suf is required in the oocyte, but does not exclude that it has critical role in other organs, which we did not observe.

Since we did not observe a mutant phenotype outside the germline, we addressed whether the p96re mutation causes a complete loss-of-function null-allele or forms a hypo morph. Comparison of Suf/Spastizin mRNA levels between $+/+,+/$ - and -/- ovaries showed a strong reduction after loss of one Suf/Spastizin copy in heterozygous adults, but no phenotype (Fig. 12d). By contrast, -/- mutant females showed only a minor reduction of mRNA compared to +/- heterozygotes, but eggs displayed the mutant phenotype. These data suggest that suf might also have a function in somatic tissue.

\subsection{Electron microscopy in oocyte shows endosome accumulation}

As mentioned above suf encodes a zinc finger FYVE-domain suggesting a crucial role in endocytic membrane trafficking. To investigate the role of Suf in vesicle trafficking, electron microscopy on the suf mutant and wild-type oocytes was performed (Fig. 13). In wild type oocytes (Fig. 13, left panel) vesicles fuse to make large yolk globules. These yolk globules are dormant lysosomes and can be characterized by a crystallized structure of stored yolk proteins when analyzed in higher magnification ((Fabra et al.,2006). In suf mutants, vesicle formation can be seen, but they accumulate immature without reaching the yolk globule stage (Fig. 13, right panel). This analysis indicates that the mutant oocytes are blocked in endosomal trafficking between the steps of vesicle formation and lysosomal degradation, which is also consistent with the results of the performed SDS-PAGE (see Fig. 10) and the observed opaque phenotype (see Fig. 9), probably caused by the lack of the proteolytic cleavage of yolk proteins. 

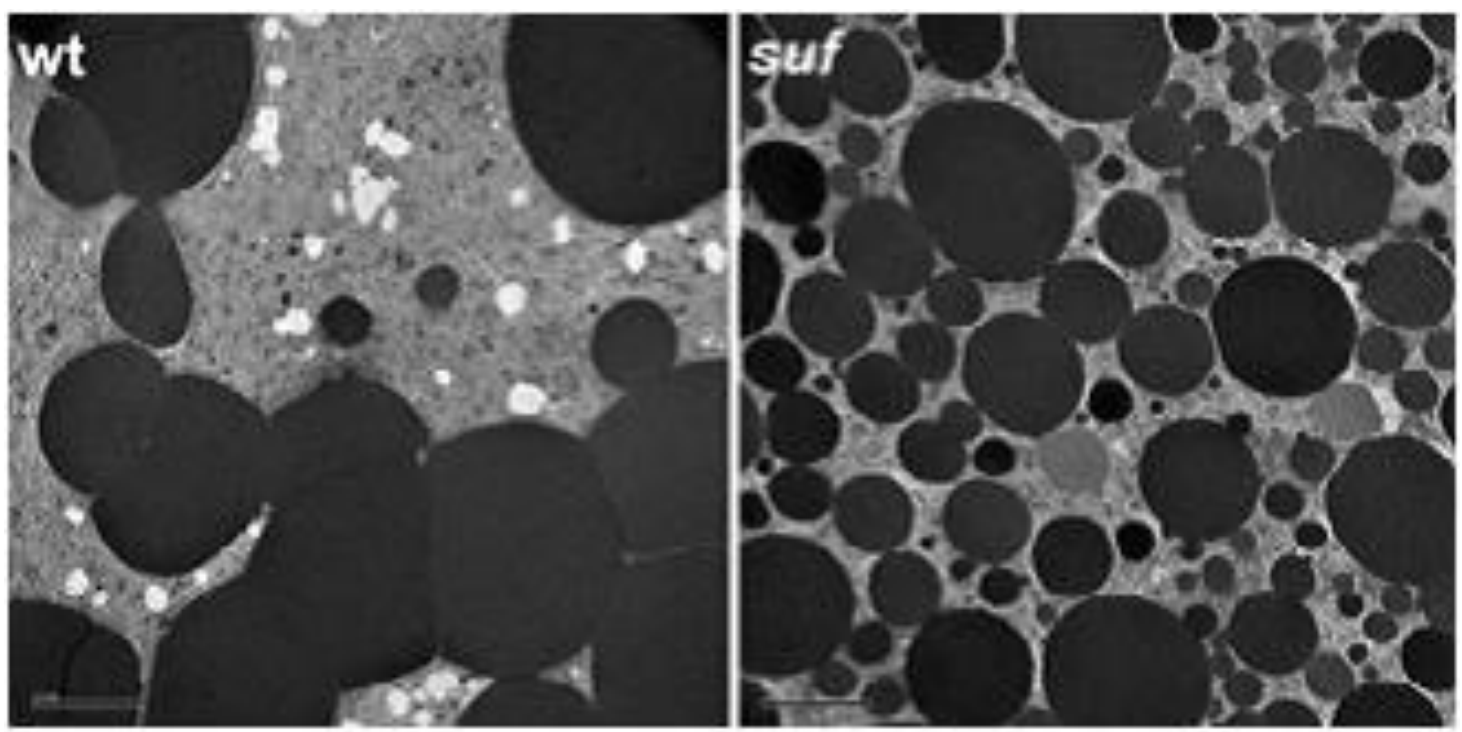

Figure 13. Suf is crucial for endosomal vesicle trafficking.

Electron micrograph of wild-type (wt) and Suf mutant oocytes. (Left panel) In wild types vesicles fuse to form large yolk globules (big grey vesicles). These vesicles are known to be dormant lysosomes. (Right panel) In mutants, the formation of vesicles can be seen but do not mature into yolk globules. Scale bar $=10 \mu \mathrm{m}$.

\subsection{Souffle mutataion causes hereditary spastic paraplegia in human.}

Interestingly, the suf mutation in human causes a genetic neuro degenerative disease called hereditary spastic paraplegia (HSP) (Hanein et al., 2008). This is a progressive motor neuron degenerative disease. It's a genetically heterogenous group of diseases with a symptom of lower limb spasticity and weakness. Hereditary Spastic Paraplegia or Strumpell-Lorrain syndrome was first described at the end of the 19th century. Human voluntary movements are under the control of the pyramidal motor system and. This system involves multiple steps: (1) signals originating from upper motor neurons, whose cell body is in the cerebral motor cortex region; (2) travel of this signal via corticospinal tract axons toward the anterior horn of the spinal cord; (3) synapses of these axons to interneurons or lower motor neurons; (4) lower motor neurons projections outside the central nervous system to the neuromuscular junctions, where they regulate skeletal muscle contraction. Thus, the impulse signal must travel a very long distance at high velocity and the cortical motor neurons need to support the very long axon with all the required components, such as proteins, lipids, mRNAs and organelles. Analysis showed that the most distal end of axon is most affected, which is most probably due to the loss of connection between upper and lower motor neurons. Moreover, the lower limbs show the symptom since they are connected through the longest axons. There are many modes of 
inheritance, with X-linked, maternal transmission (mitochondrial), autosomal recessive, autosomal dominant and de novo and the age of onset varies from early childhood to 70 years old. An uncomplicated form defined pure HSP, where lower limb spasticity occurs in isolation, frequently with bladder spasticity and mild impaired sense of vibration. Complicated HSP has prominent lower limb spasticity that is always accompanied by other neurological or non-neurological finding such as seizures, dementia, amyotrophy, ataxia, intellectual disability, deafness, extrapyramidal disturbance, thin corpus callosum, visual dysfunction, peripheral neuropathies, facial dysmorphism, persistent vomiting, orthopedic abnormality, skin lesions and much more (Noreau et al.,2014). Currently total of 54 loci has been found to cause this disease and the known genes are involved many different biological and cellular processes making more difficult to find the reason or cause for this disease (Fig.14). This tissue specific and late onset of phenotype makes it difficult to create any disease models to study the function of these disease-causing genes. The fibroblast from the patient does not show any defect while the motor neuron shows severe defects and spasticity. Many studies are aiming for identification of new HSP genes to identify the cause of the many remaining unexplained cases, as well as the downstream ones that are clinically relevant applications, such as improved diagnosis and treatments. There are several labs and organizations, which are working to (1) improved diagnostic and patient evaluation algorithms for clinician; (2) establishing national databases, registries and biobank of HSP patients; and (3) developing better treatments. So the defect in suf mutant oocyte provides an excellent tool or model to study the role of souffle in trafficking and will help to understand the disease pathology. Notably, the p96re allele is almost identical to one of the HSP mutations in human (Hanein et al.,2008).

\section{Figure 14. Pathogenic mechanisms involved in hereditary spastic paraplegia (HSP).}

This representation is a motor neuron showing where the HSP-predisposing genes are involved. These proteins can be grouped into nine different cellular functions: endosomal trafficking; DNA repair; ATP metabolism and mitochondrial localization; Fatty-acid, phospholipid and cholesterol metabolism; Autophagy and lysosomal metabolism; Gap junction; Axonal transport; Myelination and ER morphogenesis (Noreau et al.,2014). 


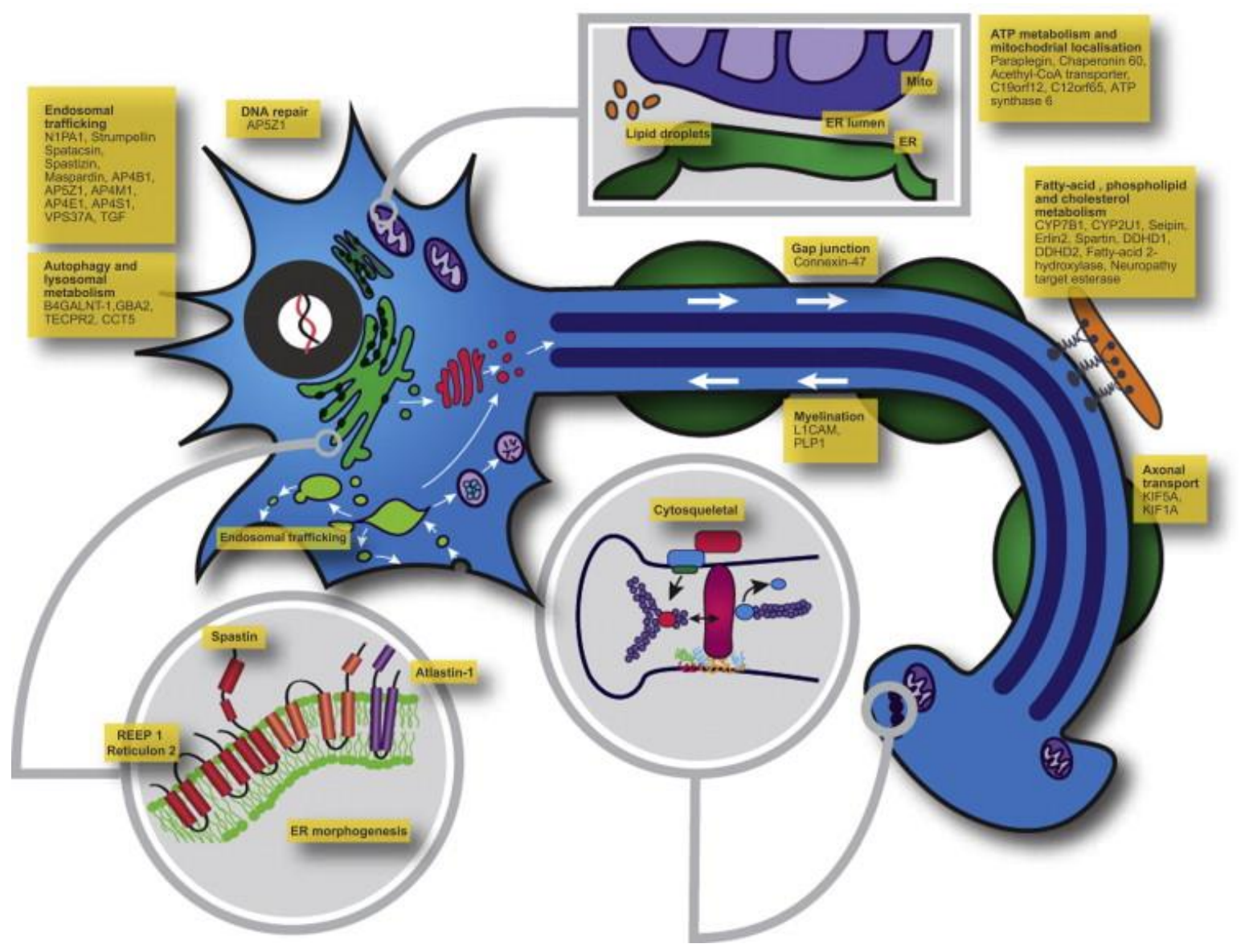

\subsection{Souffle interacts with AP5 adaptor complex in human}

Although, suf has not been studied in a model organisms, recent studies from human cells showed that suf proteins interact with newly discovered adaptor protein complex 5 (AP5) with all the sub unit of the AP5 complex together with SPG11 another HSP gene. Localization analysis showed that these proteins localize to lysosome or late endosome (Fig.15) and knockdown any of these genes causes accumulation of mannos 6phosphate receptor in endosomes. Based on bioinformatics prediction, they suggest that SPG15 protein acts as a scaffold to keep the AP5 on membrane and involves in sorting of cargos (Hirst et al.,2013a). Also the AP5 subunit has been found mutated in HSP patients. The understanding of this complex is hampered with its low abundance and absence in many model organisms. Furthermore, the depletion of SPG15 leads to disassembly of AP5 complex suggesting that SPG15 holds the complex with the membrane like a scaffold. SPG15 has been shown to localize in endosomes, ER, central nervous system, lysosome, mitochondria, centrosome and midbody (Sagona et al., 2010, 2011 \& Marmu et al., 2011). These multiple localization results also makes it hard to predict its role. However, the adaptor protein complexes are known to involve in sorting and budding of cargo from one 
compartment to another. Interaction between SPG15 and AP5 indicate their role in sorting of cargos. However, the origin and the destination of the complex is still unknown. With the suf mutant oocyte, we aim to find the function of SPG15 in endosomal trafficking to understand this complex disease.

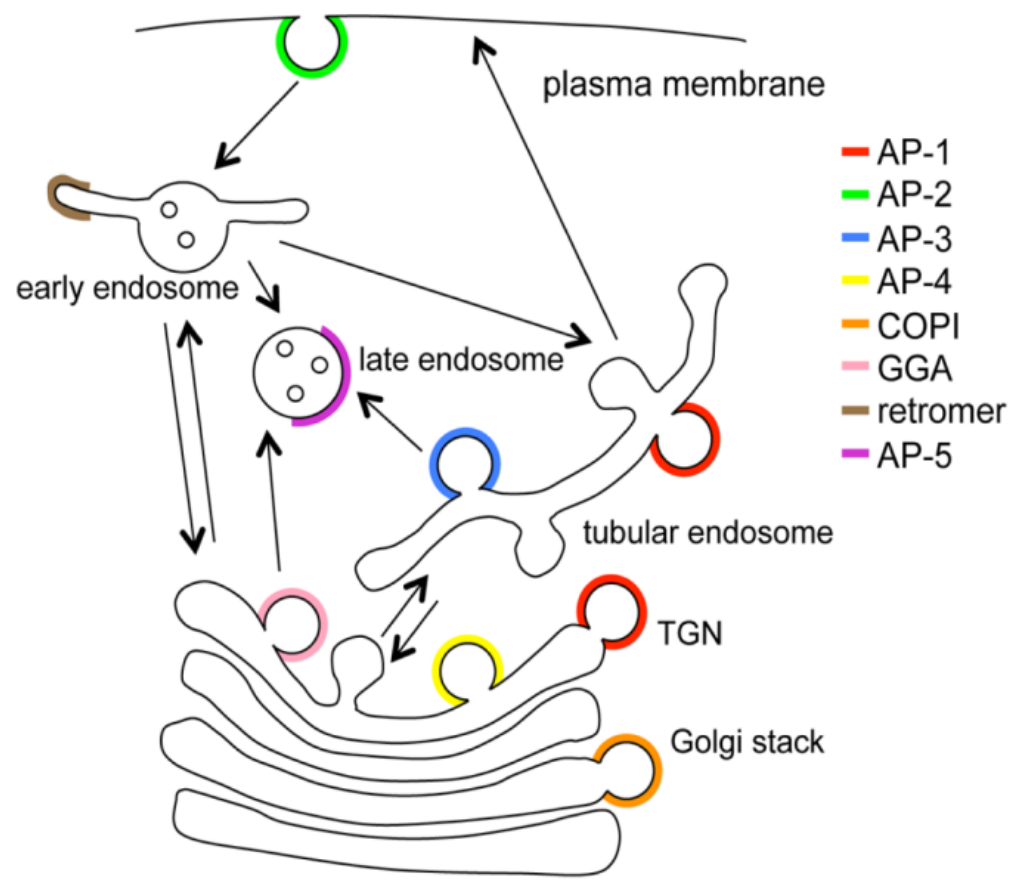

Figure 15. Adaptor protein complex 5.

The cartoon shows the localization of different adaptor proteins known and the newly discovered AP5 in late endosome. AP1 is in red in TGN, AP2 in green on plasma membrane, AP3 is in blue on tubular endosome, AP4 in yellow on TGN, GGA1 on TGN and retromer in brown on early endosome and COP1 on Golgi.

Based on the suf mutant morphological phenotype and cellular phenotype in electron micrograph indicates that suf might play crucial role in endosomal trafficking. Furthermore, suf has been shown to interact with the novel adaptor protein complex AP5 and the mutation in suf causes a genetic neuro-degenerative disease called hereditary spastic paraplegia in human signifying the essential role of suf in cellular trafficking in other tissues as well. Using the above cellular and genetic evidences represented from other studies and the preliminary data from suf mutant oocytes, I decided my following objectives. 
Introduction

\subsection{Objectives of this study}

1. Characterizations of the soufflé mutation phenotype,

2. Discovering the role of Soufflé in endocytosis during oogenesis,

3. Understanding the role of souffle in HSP disease using suf mutant oocyte as a model. 


\section{Materials and Methods}

\subsection{Model Organism-Zebrafish}

Zebrafish (Danio rerio) were maintained under standard laboratory conditions (Chapter 1, Nüsslein-Volhard and Dahm.2002). Fishes of the AB/TLF and Tubingen strain were used as wild type fishes. In vivo experiments were performed in Zebrafish oocyte and embryos according to Goettingen university Medical school animal husbandry rules and regulations. Stages of the developing embryo were determined according to (Kimmel et al, 1995).

\subsection{Bacteria}

E.coli strain XL1-Blue (RecA1, endA1, gyrA96, thi-1, hsdR17, supE44, relA1, lac[F'proAB, Z $\Delta \mathrm{M} 15, \mathrm{Tn} 10(\text { Tetr) }]^{\mathrm{c}}$ (Stratagene)) was used during this study.

\subsection{Cell lines}

The following cell lines were used:

Human skin fibroblast cells were used for analysis and cancer cells were used for expression of Suf-GFP and mutant suf expression

\subsection{Chemicals, Buffers and Media}

\subsubsection{Chemicals}

The chemicals were purchased from the following companies: Roth (Karlsruhe), Sigma (Munich), Biomol (Hamburg), Applichem (Darmstadt), Invitrogen, Sigma and Biochrom (Berlin).

\subsubsection{Buffers and Media}

The buffers were prepared using deionized water (MiliQ).

Alkaline phosphatase buffer (APB): $100 \mathrm{mM}$ Tris- $\mathrm{HCl}$ (pH 9.5), $50 \mathrm{mM} \mathrm{MgCl} 2,100 \mathrm{mM}$ $\mathrm{NaCl}, 0.1 \%$ Tween 20

Blocking solution: $1 x$ PBS, 5\% non fat dry milk and $0.1 \%$ Tween20

CoIP buffer for II: $50 \mathrm{mM}$ Tris-HCl, $\mathrm{pH} 7.5 ; 150 \mathrm{mM} \mathrm{NaCl}, 0.5 \%$ NP-40, protease inhibitor cocktail (1 tablet per $50 \mathrm{ml}$ of the buffer, Roche)

Cystein solution: 2\% L-Cystein hydrochloride, $\mathrm{pH} 8.0$ 
Laemmli running buffer (10x): $250 \mathrm{mM}$ Tris-base, $2.5 \mathrm{M}$ Glycine, $0.1 \%$ SDS

Laemmli loading buffer (6x): $350 \mathrm{mM}$ Tris- $\mathrm{HCl} \mathrm{pH}$ 6.8, 9.3\% Dithiotreit, $30 \%$ (v/v) Glycerol, 10\% SDS, 0.02\% Bromphenolblue

Luria-Bertani (LB)-Medium: 1\% (w/v) Bacto-Trypton (DIFCO), $0.5 \%$ (w/v) yeast extract (DIFCO), $1 \%(\mathrm{w} / \mathrm{v}) \mathrm{NaCl}, \mathrm{pH} 7.5$

LB-Agar: $1.5 \%(w / v)$ agar (DIFCO) in liquid LB-medium

MEM: $100 \mathrm{mM}$ MOPS, $2 \mathrm{mM}$ EGTA, $1 \mathrm{mM} \mathrm{MgSO} 4$

MEMFA: 1x MEM with 3.7\% (v/v) Formaldehyde

Nile blue: $0.01 \%(\mathrm{w} / \mathrm{v})$ Nile blue in $0.1 \mathrm{x}$ MBS

PBS (10x): 8\% (w/v) NaCl, 2\% (w/v) KCl, $65 \mathrm{mM} \mathrm{Na}_{2} \mathrm{HPO}_{4}, 18 \mathrm{mM} \mathrm{KH}_{2} \mathrm{PO}_{4}, \mathrm{pH} 7.4$

PBST buffer: $1 x P B S$ with $0.1 \%$ Tween 20

SSC: $150 \mathrm{mM} \mathrm{NaCl}, 15 \mathrm{mM}$ Sodium citrate, $\mathrm{pH} 7.4$

TAE (Tris/Acetat/EDTA): 40 mM Tris-Acetate (pH 8.5), 2 mM EDTA

TE-Buffer: $10 \mathrm{mM}$ Tris-HCl pH 8.0, $1 \mathrm{mM}$ EDTA

Tris-HCl (pH 6.8, 7.5, 8.2, 8.8, or 9.5): $1 \mathrm{M}$ Tris- $\mathrm{HCl}, \mathrm{pH}$ adjusted with $37 \% \mathrm{HCl}$

Western blotting buffer: $3.03 \mathrm{~g}$ Tris-base, $14.4 \mathrm{~g}$ Glycine, $200 \mathrm{ml}$ methanol, $800 \mathrm{ml} \mathrm{H}_{2} \mathrm{O}$

Blue juice (gel loading buffer) (10x): 50\% glycerol, $10 \mathrm{mM} \mathrm{(5 \% )} \mathrm{bromophenol} \mathrm{blue}$

Cell culture medium: Dulbecco's modified Eagle Medium (DMEM) (Biochrom), 10\% (v/v) FCS (Biochrom), $100 \mathrm{U} / \mathrm{ml}$ penicillin (Gibco Invitrogen), $100 \mu \mathrm{g} / \mathrm{ml}$ streptomycin (Gibco, Invitrogen). 


\section{Oocyte Ringer's Solutions:}

\section{Normal}

$116 \mathrm{mM} \mathrm{NaCl}$

$2.9 \mathrm{mM} \mathrm{KCl}$

$1.8 \mathrm{mM} \mathrm{CaCl} 2$

$5 \mathrm{mM}$ HEPES, pH 7.2.

\section{High calcium}

$116 \mathrm{mM} \mathrm{NaCl}$

$2.9 \mathrm{mM} \mathrm{KCl}$

$10 \mathrm{mM} \mathrm{CaCl} 2$

5 mM HEPES, pH 7.2.

Calcium free

$116 \mathrm{mM} \mathrm{NaCl}$

$2.9 \mathrm{mM} \mathrm{KCl}$

5 mM HEPES, pH 7.2.

\section{Hank's Stock Solutions (HBSS)}

Hank's buffered salt solution (HBSS) is a complicated solution to make which is why many people just buy it. First, you make each solution, the premix, and then the final solution. All solutions should be stored at $4^{\circ} \mathrm{C}$.

\section{Stock \#1}

1. Dissolve the following in $90 \mathrm{ml}$ of distilled $\mathrm{H}_{2} \mathrm{O}$

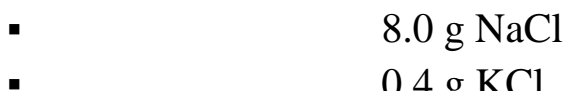

- 1.0 g glucose

2. qs to $100 \mathrm{ml}$ with distilled $\mathrm{H}_{2} \mathrm{O}$

\section{Stock \#2}

1. Dissolve the following in $90 \mathrm{ml}$ of distilled $\mathrm{H}_{2} \mathrm{O}$

- $0.358 \mathrm{~g} \mathrm{Na}_{2} \mathrm{HPO}_{4}$ (anhydrous)

- $\quad 0.60 \mathrm{~g} \mathrm{KH}_{2} \mathrm{PO}_{4}$

2. qs to $100 \mathrm{ml}$ with distilled $\mathrm{H}_{2} \mathrm{O}$

\section{Stock \#3}

1. Add $0.72 \mathrm{~g} \mathrm{CaCl}_{2}$ to $50 \mathrm{ml}$ of distilled $\mathrm{H}_{2} \mathrm{O}$ 


\title{
Stock \#4
}

1. Add $1.23 \mathrm{~g} \mathrm{MgSO}_{4} \times 7 \mathrm{H}_{2} \mathrm{O}$ to $50 \mathrm{ml}$ of distilled $\mathrm{H}_{2} \mathrm{O}$

\section{Stock \#5}

1. Add $0.35 \mathrm{~g} \mathrm{NaHCO}_{3}$ to $10 \mathrm{ml}$ of distilled $\mathrm{H}_{2} \mathrm{O}$

\section{Hank's Buffered Salt Solution (HBSS) Premix}

Combine the solutions in following in order:

$10.0 \mathrm{ml}$ Solution \#1

$1.0 \mathrm{ml}$ Solution \#2

$1.0 \mathrm{ml}$ Solution \#3

$86.0 \mathrm{ml}$ distilled $\mathrm{H}_{2} \mathrm{O}$

$1.0 \mathrm{ml}$ Solution \#4

Hank's Buffered Salt Solution (HBSS) full strength (mix prior to use)

9.9 ml Hank's Premix

$0.1 \mathrm{ml}$ Stock \#5

\section{Hank's Buffered Salt Solution (HBSS) (Full Strength with carbonate) composition}

\author{
$0.137 \mathrm{M} \mathrm{NaCl}$ \\ $5.4 \mathrm{mM} \mathrm{KCl}$ \\ $0.25 \mathrm{mM} \mathrm{Na}_{2} \mathrm{HPO}_{4}$ \\ $0.1 \mathrm{~g}$ glucose \\ $0.44 \mathrm{mM} \mathrm{KH}_{2} \mathrm{PO}_{4}$ \\ $1.3 \mathrm{mM} \mathrm{CaCl}_{2}$ \\ $1.0 \mathrm{mM} \mathrm{MgSO}_{4}$ \\ $4.2 \mathrm{mM} \mathrm{NaHCO}_{3}$
}

E3 medium: $300 \mathrm{mM} \mathrm{NaCl}, 10.2 \mathrm{mM} \mathrm{KCl}, 19.8 \mathrm{mM} \mathrm{CaCl}_{2}, 19.8 \mathrm{mM} \mathrm{MgSO}_{4}$

PCR buffer (10x): $100 \mathrm{mM}$ Tris- $\mathrm{HCl}, \mathrm{pH} 8.3,500 \mathrm{mM} \mathrm{KCl}, 15 \mathrm{mM} \mathrm{MgCl} 2,0.01 \mathrm{mM}$ EGTA

TBE buffer (10x): 0.9 M Tris-base, 0.9 M boric acid, 0.02 M EDTA, pH 8.0

Lysis-Buffer (10x): 2,5 ml 2M Tris, pH 8.4; 8,3 ml 3M KCl, 0,75 ml 1M MgCl 2 , 
Table 1. Lysis and PCR buffers

Lysis and PCR buffers:

\begin{tabular}{|l|l|l|l|}
\hline Stock solutions & $10 \mathrm{x}$ lysis buffer & $10 \mathrm{x}$ PCR buffer & Final concentration. \\
\hline $2 \mathrm{M}$ Tris 8.4 & $2.5 \mathrm{ml}$ & $2.5 \mathrm{ml}$ & $100 \mathrm{mM}$ Tris 8.4 \\
$3 \mathrm{M} \mathrm{KCl}$ & $0.75 \mathrm{ml}$ & $8.3 \mathrm{ml}$ & $500 \mathrm{mM} \mathrm{KCl}$ \\
$1 \mathrm{M} \mathrm{MgCl} 2$ & - & $0.75 \mathrm{ml}$ & $15 \mathrm{mM} \mathrm{MgCl} 2$ \\
$1 \%$ gelatin & - & $0.5 \mathrm{ml}$ & $0.01 \%$ gelatin \\
$20 \% \mathrm{BSA}(200 \mathrm{mg} / \mathrm{ml})$ & - & $250 \mu \mathrm{mg} / \mathrm{ml} \mathrm{BSA}$ \\
$\mathrm{mQ} \mathrm{H} 2 \mathrm{O}$ complete & to $50 \mathrm{ml}$ & to $50 \mathrm{ml}$ & $1 \mathrm{~m}$ \\
\hline
\end{tabular}

\section{Proteinase K:}

Stock solution: $20 \mathrm{mg} / \mathrm{ml}$. Store at $-20^{\circ} \mathrm{C}$.

Working solution: $0.2 \mathrm{mg} / \mathrm{ml}$ of lysis buffer.

\begin{tabular}{|l|l|l|}
\hline Stock solutions & $100 \mu 1$ Lysis buffer 1x & $1 \mathrm{ml}$ Lysis buffer 1x \\
\hline 10x Lysis buffer & $10 \mu \mathrm{l}$ & $100 \mu \mathrm{l}$ \\
Prot. K $20 \mathrm{mg} / \mathrm{ml}$ & $1 \mu \mathrm{l}$ & $10 \mu \mathrm{l}$ \\
mQ H2O & $90 \mu \mathrm{l}$ & $890 \mu \mathrm{l}$ \\
\hline
\end{tabular}

\section{Bromophenol blue:}

10x stock solution: $4 \%$ BP blue in $\mathrm{H} 2 \mathrm{O}$. Store at $-20^{\circ} \mathrm{C}$.

\begin{tabular}{|l|l|l|}
\hline Stock solutions & $10 x$ Loading buffer $(10 \mathrm{ml})$ & Final concentration \\
\hline $4 \%$ BP blue & $1 \mathrm{ml}$ & $0.4 \%$ BP blue \\
Glycerol & $5 \mathrm{ml}$ & $50 \%$ Glycerol \\
mQ H2O & $4 \mathrm{ml}$ & \\
\hline
\end{tabular}

Load on gel $10 \mu 1$ PCR reaction $+2 \mu 1$ 10x LB. 
dNTPs:

Prepare $500 \mu \mathrm{l}$ of $5 \mathrm{mM}$ dNTPs.

Each dNTP stock solution is $100 \mathrm{mM}$.

\begin{tabular}{|l|l|}
\hline Stock solutions & $5 \mathrm{mM}$ dNTPs $(500 \mu \mathrm{l})$ \\
\hline dATP $100 \mathrm{mM}$ & $25 \mu \mathrm{l}$ \\
dGTP $100 \mathrm{mM}$ & $25 \mu \mathrm{l}$ \\
dTTP $100 \mathrm{mM}$ & $25 \mu \mathrm{l}$ \\
dCTP $100 \mathrm{mM}$ & $25 \mu \mathrm{l}$ \\
mQ H2O & $400 \mu \mathrm{l}$ \\
\hline
\end{tabular}

\section{0 bp Ladder:}

The stock solution is $50 \mathrm{ng} / \mu \mathrm{l}$ : mix $100 \mu \mathrm{l}$ of it $+10 \mu \mathrm{l}$ of $10 \mathrm{x}$ LB. Use $3 \mu 1$ on gel.

\section{Primers:}

1 pair of primers: 1 forward primer +1 reverse primer $=1$ genomic marker The mapping pairs of primers stock are $100 \mu \mathrm{M}$ and stored at $-80^{\circ} \mathrm{C}$.

The working solution of genomic marker is $10 \mu \mathrm{M}$.

\begin{tabular}{|l|l|}
\hline Stock solutions & $10 \mu \mathrm{M}$ marker $(100 \mu \mathrm{l})$ \\
\hline Forward primer $100 \mu \mathrm{M}$ & $10 \mu \mathrm{l}$ \\
Reverse primer $100 \mu \mathrm{M}$ & $10 \mu \mathrm{l}$ \\
mQ H2O & $80 \mu \mathrm{l}$ \\
\hline
\end{tabular}

\subsection{Constructs}

\subsubsection{Vectors}

pCS2+ vector (Stratagene) 
The pCS2+ vector is a multipurpose expression vector, which can be used in the zebrafish model system. It contains a strong promoter/enhancer region (simian CMV IE94) followed by a polylinker and SV40 viral polyadenylation signal. An SP6 viral promoter is localized to the 5'-untranslated region (UTR) of the vector and enables in vitro of transcription sense mRNA for microinjection derived from of sequences cloned into the polylinker. The inserted T7 viral promoter localized to the $3^{\prime}$ UTR allows in vitro transcription of antisense RNA for in situ hybridization. The vector backbone is from the pBluescript II KS+ vector and includes the ampicillin resistance gene and an f1 origin for producing single stranded DNA.

\section{pBluescripts KS (-) vector (pBS) (Stratagene)}

The pBluescript vector is a phagemid vector designed for DNA cloning and sequencing procedures. KS represents the orientation of the MCS in which lacZ transcription proceeds restriction sites from SacI to KpnI. The phagemid contains the intergenic region of phage 11 required for packaging of DNA into bacteriophage particles, the $\mathrm{T} 3$ and $\mathrm{T} 7$ promoter for in vitro transcription, the bla (ApR) - gene, coding for betalactamase, that confers resistance to ampicillin, lac $Z$ - the $5^{\prime}$-terminal part of the lac $Z$ gene encoding the N-terminal fragment of beta-galactosidase. This fragment allows blue/white screening of recombinant phagemids

\section{pCS2+_YFP_rab4a}

pCS2+_YFP_rab4a is a pCS2+ vector variant containing the open reading frame of $Y F P$ fused to the open reading frame of $r a b 4 a$ for the expression of a reporter gene.

\section{pCS2+_CFP_rab5a}

This vector harbors the open reading frame encoding for CFP fused to the $\mathrm{N}$ terminus of $r a b 5 a$ for the expression of a reporter construct.

\section{pCS2+_YFP-rab7}

The coding sequence of YFP linked with that of $r a b 7$ is inserted into the pCS2+ vector for the expression of a fusion protein.

\section{pCS2+_CFP_rab11a}


This vector is a variant of the pCS2+ vector containing the cDNA sequence of $C F P$ connected to the cDNA sequence of the dog rablla for the expression of a reporter gene.

\section{pCS2+_eGFP_cellubrevin}

The pCS2+ vector harbors the open reading frames of $e G F P$ and cellubrevin fused with each other for the expression of a reporter construct.

\subsubsection{Gateway vectors}

The gateway vectors were used to transfer suf clone from pUC57 to these expression vector using recombination. In addition, we used to fuse GFP or mCherry in both ends using company recommended procedures (Invitrogen).

$$
\begin{aligned}
& \text { pCS2+ Dest egfp } \\
& \text { pCS2+ Dest } \\
& \text { pCS2+ Dest mCherry }
\end{aligned}
$$

\subsection{Oligonucleotides}

The oligonucleotides (primers) were purchased from Sigma-Aldrich Chemie and Eurogentec. They were dissolved in $\mathrm{HPLC} \mathrm{H}_{2} \mathrm{O}$ to a concentration of $100 \mu \mathrm{M}$.

\subsubsection{Sequencing primers}

The sequencing primers used during this study are listed in Table 1.

\section{Table 2. Sequencing primers}

\begin{tabular}{|l|l|l|}
\hline Construct/Vector & Name & Nucleotide-sequence $\left(5^{\prime} \square 3^{\prime}\right)$ \\
\hline pBS & T3 & AATTAACCCTCACTAAAGGG \\
\hline \multirow{2}{*}{ pCS2+ } & T7 & TAATACGACTCACTATAGGGCGA \\
\hline \multirow{2}{*}{ soufflé } & T3 & AATTAACCCTCACTAAAGGG \\
& T7 (pCS2+) & TCTACGTAATACGACTCACTATAG \\
\hline & Sp6 & TAATACGACTCACTATAGGGCGA \\
\hline & zn_finger_fw & TGAGGTCAGACATCTTTGAG \\
\cline { 2 - 3 } & suf_new_fw & TCATGCACCCGTTCGGCCGTGAA \\
\hline
\end{tabular}




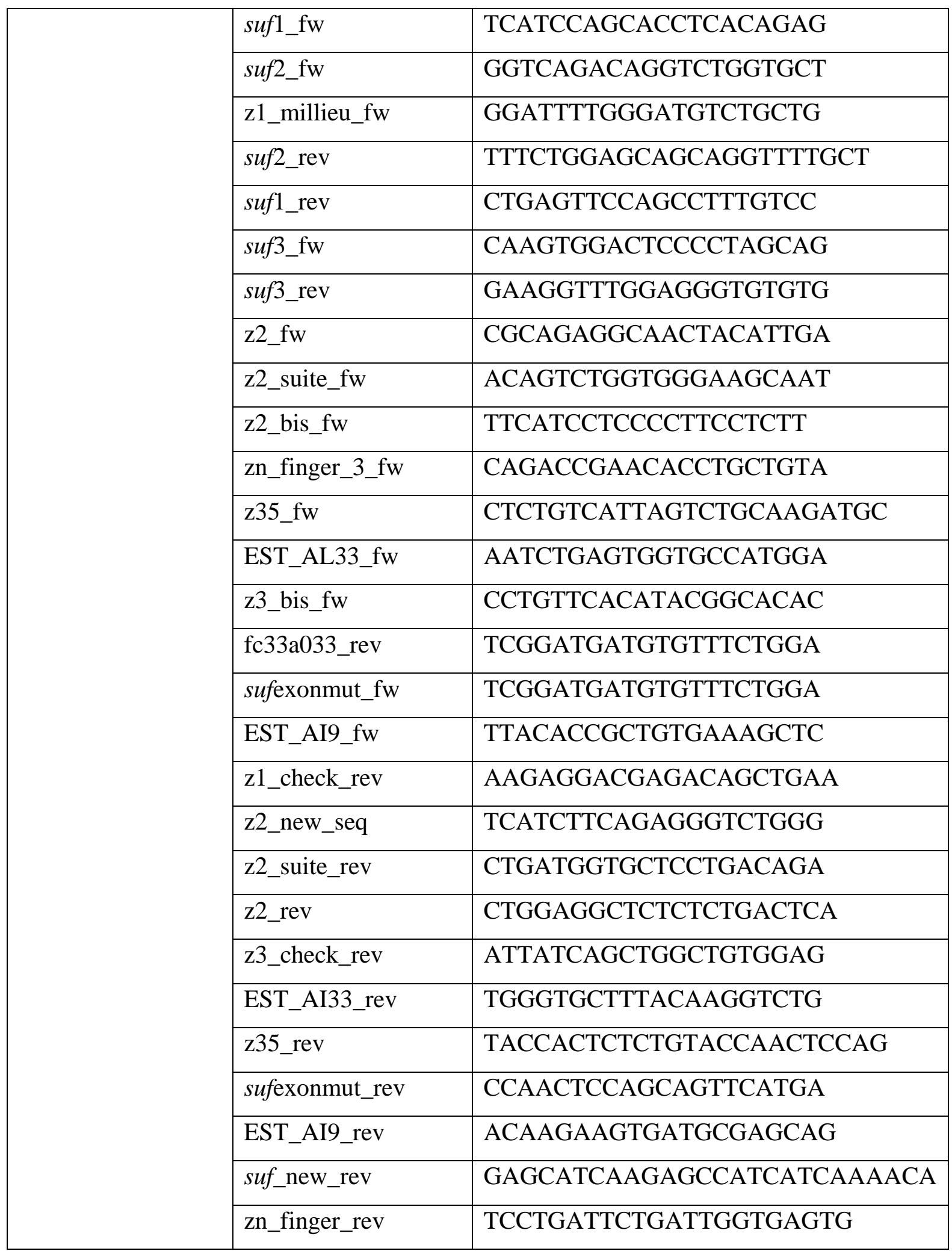

\subsubsection{Morpholino oligonucleotides}

Antisense Morpholino oligonucleotides (Morpholinos, MO) were purchased from Gene Tools, LLC (Philomath, USA). Morpholinos were dissolved in RNAse-free water to a 
$1 \mu \mathrm{M}$ concentration. The sequences of the Morpholinos used in this study are presented in Table 2.

Table 3. Antisense Morpholino oligonucleotides for suf

\begin{tabular}{|c|c|c|c|}
\hline $\begin{array}{c}\text { Morpholino } \\
\text { name }\end{array}$ & $\begin{array}{l}\text { Target } \\
\text { gene }\end{array}$ & Sequence $5^{\prime} \rightarrow 3^{\prime}$ & $\begin{array}{c}\text { Working } \\
\text { concentration }\end{array}$ \\
\hline Suf & Suf ATG & $\begin{array}{l}\text { AUGMO- } \\
\text { GCCGAACGGGTGCATGATCCTCAAA }\end{array}$ & $\begin{array}{l}\text { 5-10 } \\
\text { ng/embryo }\end{array}$ \\
\hline Control & No target & $\begin{array}{l}\text { Control MO- } \\
\text { GCCcAACcGcTGCATcATgCTCAAA }\end{array}$ & $\begin{array}{l}5-10 \\
\text { ng/embryo }\end{array}$ \\
\hline
\end{tabular}

\subsection{DNA methods}

\subsubsection{Plasmid DNA preparations}

Isolation of plasmid DNA in analytical amounts was performed using Illustra ${ }^{\mathrm{TM}}$ Plasmid Prep Mini Spin Kit (GE Healthcare). For the isolation of plasmid DNA in preparative amounts Illustra ${ }^{\mathrm{TM}}$ Plasmid Prep Midi Flow Kit (GE Healthcare) was used. The DNA isolation was performed according to the manufacturer's instructions. DNA concentrations were measured using the ND-1000 Spectrophotometer, Coleman Technologies Inc.

\subsubsection{DNA restriction digestion}

DNA restriction digestion was performed with restriction endonucleases purchased from MBI Fermentas according to manufacturer's instructions.

\subsubsection{Agarose gel electrophoresis}

DNA or RNA fragments were separated in a horizontal electrical field into agarose gel (Sharp et al.,1973). The electrophoresis was run in the standard TAE-running buffer at $100-120 \mathrm{~V}$ in horizontal electrophoresis chamber. Depending on the expected sizes of DNA/RNA fragments, 0.8 to $2 \%(\mathrm{w} / \mathrm{v})$ agarose gels were prepared in TAE buffer. Gels always contained $0.5 \mu \mathrm{g} / \mathrm{ml}$ ethidium bromine to visualize nucleic acids. Before loading samples into the gel slots, nucleic acids were mixed with DNA loading dye (6x, Ambion). After the electrophoresis, DNA bands were visualized with the UV-transilluminator 
(Herolab) and documented with the ChemiDoc video documentation system (EASY view). Standard DNA ladders were used to determine the sizes of DNA fragments (High, Middle or Low Range, Fermentas).

\subsubsection{Purification of DNA fragments from agarose gel or restriction digestion mixture}

The purification of DNA fragments from agarose gels or restriction digestion mixture was performed with the Illustra ${ }^{\mathrm{TM}}$ GFX PCR DNA and Gel Band Purification Kit (GE Healthcare) according to manufacturer's instructions.

\subsubsection{Polymerase chain reaction (PCR)}

A standard PCR reaction was used to amplify desired DNA fragments (Mullis et al.,1986).

The PCR reaction mixture:

Matrix DNA - $50 \mathrm{ng}$

10x buffer $-5 \mu 1$

dNTP (10mM each, Fermentas) $-2 \mu 1$

DNA polymerase $-1 \mu 1$

$10 \mu \mathrm{M}$ forward primer $-1 \mu \mathrm{l}$

$10 \mu \mathrm{M}$ reverse primer $-1 \mu \mathrm{l}$

HPLC water (Roth) - up to $50 \mu 1$

DreamTag $^{\text {TM }}$ polymerase $(5 \mathrm{U} / \mu \mathrm{l})$ (Fermentas) was used for analytical PCR. High fidelity Pfu polymerase (2.5 U/ $\mu 1$, Fermentas) was used for the PCR followed by molecular cloning of the amplified fragments.

The following program was used for DNA fragments amplification:

1 cycle:

Initial denaturation step $-95^{\circ} \mathrm{C}, 2 \mathrm{~min}$

30 cycles:

Denaturation $-95^{\circ} \mathrm{C}, 45 \mathrm{sec}$

Primer annealing - the temperature depends on primer GC content, $45 \mathrm{sec}$

Elongation step $-68^{\circ} \mathrm{C}$ for $P f u$ polymerase, $72^{\circ} \mathrm{C}$ for DreamTag ${ }^{\mathrm{TM}}$ polymerase, $1 \mathrm{~min} / 1000 \mathrm{bp}$ of target DNA fragment

1 cycle:

Final elongation step $-68^{\circ} \mathrm{C}$ or $72^{\circ} \mathrm{C}$ depending on the polymerase used, $10 \mathrm{~min}$ 


\subsubsection{DNA ligation}

T4 DNA ligase (Fermentas) was used according to manufacturer's instructions. For the ligation, a vector and an insert were taken in 1 to 3 molar ratio. The total amount of vector DNA was $50 \mathrm{ng}$. For $20 \mu \mathrm{l}$ of a single reaction, mixture $2 \mu \mathrm{l}$ of T4 DNA ligase (5 $\mathrm{U} / \mu \mathrm{l})$ was used. The ligation was performed 1 hour at room temperature for inserts shorter than $1000 \mathrm{bp}$ or overnight at $16^{\circ} \mathrm{C}$ for inserts longer than $1000 \mathrm{bp}$. The ligase was inactivated $10 \min 65^{\circ} \mathrm{C}$ before the transformation of E.coli Xl1blue strain.

\subsubsection{Chemical transformation and electro-transformation of bacterial cells}

For chemical transformation $100 \mu \mathrm{l}$ of chemically competent E.coli XL1blue cells were thawed on ice, mixed with 1 or $2 \mu$ of the ligation mix or 100-500 ng of plasmid DNA, incubated for $10 \mathrm{~min}$ on ice and heat-shocked for $2 \mathrm{~min}$ at $41^{\circ} \mathrm{C}$, then left for $2 \mathrm{~min}$ on ice. $1 \mathrm{ml}$ of warm LB medium was added to the cells and the mixture was incubated at $37^{\circ} \mathrm{C}$ for 1 hour (Mandel and Higa.1970). For electro-transformation $40 \mu \mathrm{l}$ of electrocompetent cells were thawed on ice, mixed with 1 or $2 \mu$ of the ligation mix, transferred into a yellow $2 \mathrm{~mm}$ electroporation cuvette (Thermo Electron) and incubated for $1 \mathrm{~min}$ on ice. After application of an electrical pulse of $1.8 \mathrm{kV}$ and $25 \mu \mathrm{F}$, the transformation reaction was mixed with $0.5 \mathrm{ml}$ of LB-medium (Dower et al.,1988). Bacterial pellets were seeded on LB agar plates supplemented with appropriate antibiotics for the selection of transformed cells $(0.1 \mathrm{mg} / \mathrm{ml}$ ampicillin (Biomol), $0.05 \mathrm{mg} / \mathrm{ml}$ kanamycin (Biomol). Colonies were grown overnight at $37^{\circ} \mathrm{C}$ (Mandel and Higa.1970).

\subsubsection{DNA sequencing analysis}

Dye-termination sequencing method, which is the modification of Sanger chaintermination sequencing, was used (Sanger et al.,1977). The Big Dye ${ }^{\mathrm{TM}}$ Terminator Kit (Applied Biosystems) was used for preparation of the sequencing PCR according to the manufacturer's instructions.

\section{The sequencing PCR mixture:}

DNA matrix - 200-400 ng

Seq $\operatorname{mix}-1.5 \mu 1$

Seq buffer $-1.5 \mu 1$ 
Primer -8 pmole

HPLC water - up to $10 \mu l$

\section{The sequencing PCR}

25 cycles:

$96{ }^{\circ} \mathrm{C}, 10 \mathrm{sec}$

$55^{\circ} \mathrm{C}, 15 \mathrm{sec}$

$60^{\circ} \mathrm{C}, 40 \mathrm{sec}$

To purify the sequencing reaction the following components were added to the PCR mixture: $1 \mu \mathrm{l}$ of $125 \mathrm{mM}$ EDTA ( $\mathrm{pH} 8.0), 1 \mu \mathrm{l}$ of $3 \mathrm{M}$ sodium acetate ( $\mathrm{pH} 5.4)$ and $50 \mu \mathrm{l}$ $100 \%$ ethanol. The sample was incubated $5 \mathrm{~min}$ at room temperature, and then centrifuged $15 \mathrm{~min}$ at $14000 \mathrm{rpm}$. The pellet was washed with $70 \mu \mathrm{l}$ of $70 \%$ ethanol, dried and diluted in $15 \mu \mathrm{l}$ of $\mathrm{HiDi}^{\mathrm{TM}}$ buffer (Applied Biosystems). The automated sequencing was performed by in-house sequencing lab using the ABI 3100 Automated Capillary DNA Sequencer (Applied Biosystems).

\subsection{RNA methods}

\subsubsection{In vitro synthesis of capped sense mRNA}

Prior to capped sense mRNA synthesis $15 \mu \mathrm{g}$ plasmid DNA were digested at $37{ }^{\circ} \mathrm{C}$ overnight in $40 \mu$ l total volume via FastDigest ${ }^{\circledR}$ KpnI (1 FDU/ $\mu$ L, Fermentas). The linearized plasmid was either purified using Invisorb ${ }^{\circledR}$ Fragment CleanUp (Invitek) (Exp. 2) or extracted with phenol/chloroform/isoamyl alcohol (Roth) (Exp.1). Prior to the extraction the linearized plasmid was treated with $0,3 \mu \mathrm{g}$ proteinase $\mathrm{K}(20 \mathrm{mg} / \mathrm{ml}, \mathrm{MP})$ for $30 \mathrm{~min}$ at $55^{\circ} \mathrm{C}$. Thereafter, phenol/chloroform/isoamyl alcohol (1:1) was added, the tube was vortexed and centrifuged for one min at $16.000 \mathrm{rcf}$. The upper phase was precipitated with $1 / 2$ volume $5 \mathrm{M} \mathrm{NH}_{4} \mathrm{Ac}$ and two volumes $100 \%$ ethanol via centrifugation for $15 \mathrm{~min}$ at $16.000 \mathrm{rcf}$ at RT. Afterwards, the supernatant was removed and the pellet was washed with $70 \%$ ethanol for five min at 16.000 rcf. Finally, the Pellet was re-suspended in nuclease free $\mathrm{H}_{2} \mathrm{O}$.

In vitro synthesis of capped sense mRNA for microinjections into Zebrafish embryos was performed using the Sp6 mMESSAGE mMACHINE SP\& kit (Ambion) 
according to the manufacturer's instructions. For a $20 \mu \mathrm{l}$ reaction $0.5 \mu \mathrm{g}$ of the linearized plasmid was used as template DNA. The reaction took place for 2 hours at $42{ }^{\circ} \mathrm{C}$. Subsequently, $1 \mu \mathrm{l}$ of TURBO DNase (2U/ $\mu 1$, Ambion) was added to remove the template DNA. Synthesized RNA was purified via the Illustra ${ }^{\mathrm{TM}}$ ProbeQuant $^{\mathrm{TM}}$ G-50 Micro Columns kit (GE Healthcare)

\subsubsection{Measurement of the RNA concentrations}

RNA concentrations were measured using ND-2000c Spectrophotometer (PEQLAB Biotechnologie $\mathrm{GmbH}$ ). For RNA, an optical density (OD) of 1 corresponds to $40 \mu \mathrm{g} / \mathrm{ml}$.

\subsection{Zebrafish embryo culture and microinjections}

\subsubsection{Setting up pair crosses}

Male and female Zebrafish pairs were set up in mice cages in the afternoon using the same conditions under which the fish are otherwise maintained. Males were separated from the females by an inserted mating box containing a mesh divider to obtain precise staging of the embryos. The following morning, upon light cycle activation, the pairs were put together into the mating boxes, and the females were stimulated to release mature eggs. Embryos were run through the mesh divider and could thus easily separated from their parents and collected. Embryos were raised in E3 buffer.

\subsubsection{Embryo microinjection of capped sense mRNA}

\subsubsection{Needle preparation}

The injection needles were made from Borosilicate glass with firepolished ends with filament (GB100F-8P, SCIENCE PRODUCTS GmbH) on Magnetic Glass Microelectrode Horizontal Puller (Model PN-30, Narishige). The pulling parameters were empirically determined. A commonly used set was as follows: Heater level (61.2), Magnet main level (70.6), and Magnet sub level (27.6). The needles were further cut with a razorblade in order to make fine wedge-shaped tips.

\subsubsection{Preparation of injection dishes}

To facilitate injection procedure, embryos were held in wedge-shaped troughs. For the preparation of these troughs, liquid 1\% agarose/E3 buffer was poured into $100 \mathrm{x} 15 \mathrm{~mm}$ petri dishes and plastic molds (chamfer down) were set to agarose/E3 overlay. Once agarose/E3 was solidified, the plastic molds were removed and the petri dishes were kept under $4{ }^{\circ} \mathrm{C}$ cold conditions to prevent bacteria growing. 


\subsubsection{Microinjection into 1-cell stage embryos}

Before injection, the synthesized capped sense mRNA was diluted with $0.05 \%$ phenol red solution (Sigma) and $0.1 \mathrm{M} \mathrm{KCl}$ to the final concentration. This solution was loaded into the needles. The microinjection was performed as described in 'Zebrafish - A practical approach: chapter 5' (Nüsslein-Volhard and Dahm, Chapter 5, 2002). The following RNAs were injected: YFP-rab4, CFP-rab5a, YFP-rab7, CFP-rab11a, eGFPcellubrevin, mcherry, mcherry-soufflé_A3.

\subsubsection{Morpholino injection of one cell embryo}

One cell embryo was injected with previously described amount in Martin et al., 2012. Injected embryos were cultured for two days and chcked for their development and sored for the phenotype observed.

\subsection{Oocyte methods}

\subsubsection{Oocyte isolation and culture}

The gravid females were anesthetized and ovary were dissected using thin blade under the belly. Keep the ovaries in OR2 buffer without disturbing and pas through the filters to isolate individual oocytes. Then slowly move the column up and down inside the buffer to collect the single separated oocytes from the ovary. We can also use enzymes which digest the ovaries (Collagenase 1A $15 \mu \mathrm{g} / \mathrm{ml}$ in R2 buffer) or Liberase (9 $\mu 1$ of 14 wünsch/ml) for shorter time, followed by washing to remove the enzymes then collect the single oocytes. But don't destroy or damage the oocytes by doing faster. Then wash the oocytes twice with R2 buffer or 60\% L-15 media. The oocytes are selected based on their size using the filters with different sizes according to different stage of the oocytes in 8 well plates (Available in the lab). Isolated oocytes can be used further for injection or drug treatment followed by culture or imaging purpose.

\subsubsection{In-vivo Trafficking assay}

The oocytes were dissected out from the female from wild type and suf mutant in OR2 buffer and Incubated with $125 \mu \mathrm{g} / \mathrm{ml}$ of Transferrin Alexa594 (Molecular probes) in OR2 buffer for 10 and 25 minutes at room temperature followed by 30 minutes of chasing in OR2 buffer alone. The oocytes were fixed after washing twice with OR2 and twice with PBT and stained with antibodies or fluorescent dyes. The oocytes were isolated from wild type and suf mutant females and incubated with $10 \mu \mathrm{g} / \mathrm{ml}$ of LDL Dil (Molecular probes) 
in OR2 buffer for 10 minutes then the oocytes were fixed after washing twice with OR2 and twice with PBT followed by lysotracker staining.

\subsubsection{Chorion elevation assay}

\subsubsection{Chorion elevation assay in egg}

The fish were set in mating box but the male and female were separated by a mesh to prevent mating in previous day evening. The fish were anesthetized using tricane solution and the ovulated eggs were squeezed out from wild type, suf heterozygous and suf homozygous female in OR2 buffer or hank solution. The eggs were activated by adding E3 medium and left it for 30 mins. The eggs were imaged after 30 minutes of activation. The images were used to measure the chorion elevation level using Fiji software.

\subsubsection{Chorion elevation assay in embryo}

The fish were set for mating but the male and female were separated by a mesh in previous day evening. The fish were put together in the morning for mating. After 20 mins of undisturbed mating time, the embryos were collected from wild type, suf heterozygous and homozygous female. The embryos were imaged after 30 minutes post fertilization and measured their chorion elevation using Fiji software.

\subsubsection{Dynasore teatement}

The live oocytes or eggs were collected in OR2 buffer and incubated with 500 $\mu \mathrm{g} / \mathrm{ml}$ of Dynasore for required time at room temperature. The control oocytes were incubated with the carrier DMSO. Then, the oocytes were washed thrice and processed further for staining as mentioned in immunofluorescence staining or functional assay for chorion elevation.

\subsubsection{Electron Microscopy}

Electron Microscopy on oocytes was done in collaboration with Dr. Dietmar Riedel Max Planck institute of Biophysical Chemistry, Goettingen. The Diameter was measured in $\mathrm{nm}$ and standard deviation was used to put error bar in the graph and t-test was done in MS-Excel and WWW.graphpad.com.

For high-pressure freezing EM, living oocytes were placed in aluminum platelets of $150 \mu \mathrm{m}$ depth containing 1 hexadecen (Studer et al.,1989). The platelets were frozen using a Leica Em HPM100 high-pressure freezer (Leica Mikrosysteme Vertrieb GmbH, Wetzlar, Germany). The frozen oocytes were transferred to an automatic Freeze Substitution Unit Leica EM AFS2. The samples were substituted at $-90{ }^{\circ} \mathrm{C}$ in a solution containing 
anhydrous acetone, $0.1 \%$ tannic acid for $24 \mathrm{~h}$ and in anhydrous acetone, $2 \% \mathrm{OsO}_{4}, 0.5 \%$ anhydrous glutaraldehyde (EMS Electron Microscopical Science, Ft. Washington, USA) for additional $8 \mathrm{~h}$. After a further incubation over $20 \mathrm{~h}$ at $-20{ }^{\circ} \mathrm{C}$, samples were warmed up to $+4{ }^{\circ} \mathrm{C}$ and washed with anhydrous acetone subsequently. The samples were embedded at room temperature in Agar 100 (Epon 812 equivalent) at $60{ }^{\circ} \mathrm{C}$ over $24 \mathrm{~h}$. Images were taken in a Philips CM120 electron microscope (Philips Inc.) using a TemCam 224A slow scan CCD camera (TVIPS, Gauting, Germany).

\subsubsection{Oocyte injection with RNA or DNA}

For plasmid injection, isolated stage III oocytes were injected with $1 \mathrm{ng}$ of pCS2+wt-suf or pCS2+mut-suf encoding the p96re allele as described (Clelland et al.,2007). After injection oocytes were incubated for $12 \mathrm{hrs}$ at $28^{\circ} \mathrm{C}$ in $90 \% \mathrm{~L}-15$ medium $(0.5 \% \mathrm{BSA} ; 100 \mu \mathrm{g} / \mathrm{ml}$ Gentamycin $)$ and then scored for chorion elevation as described before (Nair et al.,2013).

\subsubsection{Microinjection and Oocyte Maturation}

Zebrafish oocytes were injected RNAs or DNAs in a volume of $1 \mathrm{~nL}(0.017 \mathrm{fmol})$ per oocyte. After $1 \mathrm{~h}$ of culture, healthy injected oocytes were cultured in $60 \% \mathrm{~L}-15$ medium with $10 \mu \mathrm{g} / \mathrm{ml} 17 \alpha, 20 \beta$-dihydroxy-4-pregnen-3-one (Sigma) at $28^{\circ} \mathrm{C}$ for $4-6 \mathrm{~h}$. Oocytes that became translucent after this treatment were harvested as mature oocytes. Those matured oocyte can be used for eg activationto check chorion elevation.

\subsubsection{Mitochondrial staining of oocytes (Nora).}

Place the ovary in 60\% L-15 medium (see Materials and methods, Buffers). Add $0,1 \mathrm{mg} / \mathrm{ml}$ liberase for 10 minutes at RT. Wash with enzyme-free L15 medium twice, Pipette the ovary up and down gently. Place samples in proteinase $\mathrm{K}$ lysis buffer (see Materials and methods, Buffers) for 3 minutes at RT. Wash twice with L-15 medium. Add the corresponding dye: TMRE (Tetramethylrhodamine ethyl ester perchlorate, Life technologies) for 10 minutes concentration $1 \mu \mathrm{M}$, JC-1 (Life technologies) for 45 minutes concentration $5 \mu \mathrm{M}$. For the CCCP (Carbonyl cyanide $m$-chlorophenyl 11 hydrazone, Life Technologies) treatment add $5 \square \mathrm{M}$ for 30 minutes before adding the dye and do not remove during the staining. Wash twice with L-15 for 20 minutes at room temperature. Image immediately, while the oocytes are still alive. 


\subsubsection{Confocal Microscopy}

Images were captured at room temperature using LSM780 confocal microscope (Carl Zeiss) with a Plan Apochromat 63x/1.4 NA and 25x/0.8 NA oil-immersion and a digital microscope camera (M27; Carl Zeiss). After washing the oocytes with PBT, yolk was cleared with Murray's solution (Benzylbenzoate (66\%)/ Benzylalcohol (33\%)) during imaging. A multiple wavelength laser was used to visualize red (561) fluorescence, green (488, 405) and blue (405) and images were acquired and processed using ZEN 2011 software (Carl Zeiss).

\subsubsection{Immunostaining of oocytes}

The oocytes were isolated from gravid females and prepared further for taining using following protocol.

\section{Day 1:}

\section{Fixation:}

- Place oocytes in glass vials and add ProteinaseK solution. Incubate for 3min @ RT

- Remove ProteinaseK solution and wash twice with 1ml MEMFA

- Rock vials for 1h @ RT in 1ml MEMFA

- Remove MEMFA and add 1ml PBT, rock @ RT for 15min, wash twice with PBT

- Replace PBT with 500 $\mu$ l fresh PBT + 2\%BSA, 2\% horse serum, and rock for 2hrs @ RT

\section{Antibodies:}

- Replace with 500 $\mu$ PBT + 2\% BSA, 2\% horse serum and th e appropriate dilution of the primary antibody

- Rocko/n@ $4^{\circ} \mathrm{C}$

\section{day2:}

- Replace primary antibody solution with 500 $\mu$ PBT and rock @ RT for 1.5hrs, wash twice with PBT

- Replace with PBT + 2\%BSA, 2\% horse serum and the appropriate solution of the secondary antibody

- Rock vials o/n@ @ ${ }^{\circ} \mathrm{C}$

day3:

- Replace solution with 500 $\mu$ PBT and rock @ RT for 1.5hrs, wash twice with PBT - For counterstaining (DAPI, lysosomal marker), replace with PBT $+2 \%$ BSA $+2 \%$ horse serum and appropriate amount of DAPI and/or marker 
-Rock @ RT for 1h

- Rock with PBT for some time, wash 2x with PBT

\section{Dehydration:}

- Replace half of the volume and replace with MeOH 3x (equal to 50\%, 75\%, 87.5\% of $\mathrm{MeOH})$

- Remove all of the solution and replace with $\mathbf{M e O H}$

- Wash with MeOH

- Oocytes can be stored @ $-20^{\circ} \mathrm{C}$ until ready to image

\section{Imaging:}

- Place Murray's Clearing Medium into a Fluorodish

- Transfer oocytes from glass vials onto imaging dish, with as little $\mathrm{MeOH}$ as possible

Murray's Clearing Medium: 2:1 Benzyl Benzoate: Benzyl alcohol

For double staining, the oocytes were incubated with Suf labeled with ATTO590 (SySy, Goettingen) for $2 \mathrm{hrs}$ at room temperature. The DNA was stained with $1 \mu \mathrm{M}$ of Hoechst 33342. The cortical granule was stained with $50 \mu \mathrm{g} / \mathrm{ml}$ of Lectin PHA-L Alexa 488 (Molecular probes) or MPA Lectin Texas Red (EY Labs Inc). The Cathepsin D Proteases were stained with $1 \mu \mathrm{M}$ Pepstatin A BODIPY FL conjugate (Molecular Probes) and Lysotracker DND-99 Red (Mol. Probes) at $70 \mathrm{~nm}$ concentration. After washing the oocytes with PBT, yolk cleared with Murray solution and imaged on a Zeiss LSM 710. Confocal data were analyzed using ZEN2010 software.

\section{Table 4. Antibodies used in this study}

\begin{tabular}{|l|l|l|}
\hline Antibody & Host & Company \\
\hline Rab5A (S-19) (sc-309) & Rabbit & SCB \\
Rab7 (ab50533) & Mouse & abcam \\
Rab11b (GTX127328) & rabbit & GENETEX \\
Suf(Fish) & Rabbit & Biogene (Custom) \\
Suf Human & Rabbit & Stevanin gift \\
Clathrin LC1 (3F133) & Mouse & abcam \\
TGN38 & Rabbit & SCB \\
Caveolin1 & Rabbit & \\
& & abcam \\
\hline
\end{tabular}




\begin{tabular}{|c|c|c|}
\hline VTG & Mouse & Genetex \\
\hline Rab9a(ZF127768) & Rabbit & genetex \\
\hline Rab35 (ZF125343) & Rabbit & genetex \\
\hline Rab4a (ZF127780) & Rabbit & genetex \\
\hline Rab4b (ZF125364) & Rabbit & Genetex \\
\hline Rab3ab (GTX127329) & rabbit & Genetex \\
\hline ATP6V0d1(GTX125970) & rabbit & Genetex \\
\hline LC3(GTX48634) & rabbit & Genetex \\
\hline Rab35 (GTX120294) & Rabbit & Genetex \\
\hline DNM2 & Rabbit & DSHB \\
\hline Znp-1 & Mouse & DSHB \\
\hline Zn-1 & Mouse & DSHB \\
\hline SV-2 & Mouse & DSHB \\
\hline Zn-8 & Mouse & DSHB \\
\hline FD59 & Mouse & DSHB \\
\hline Islet $1 \& 2$ & Mouse & SySy \\
\hline Vamp4 & Rabbit & Sigma \\
\hline Beta catenin & Mouse & Genetex \\
\hline Lamp-1 & Mouse & SCB \\
\hline V-ATPase a1 & Rabbit & Dr. Joan Cerda Gift \\
\hline Cat b & Rabbit & Dr. Joan Cerda Gift \\
\hline Cat F & Rabbit & Dr. Joan Cerda Gift \\
\hline Cat L & Rabbit & Carlo, France \\
\hline Cat D & Rabbit & Dr. Eric Jansen Netherlands \\
\hline Ac45 & Rabbit & Dr. Eric Jansen Netherlands \\
\hline POMC & Rabbit & LS bioscience \\
\hline GGA1 & Rabbit & Novus Bilogicals \\
\hline VAMP7 & Rabbit & Cologne \\
\hline M6PR 300 & Rabbit & Cologne \\
\hline M6PR 46 & Rabbit & Cologne \\
\hline M6PR 300 & Rabbit & Genetex \\
\hline BCN1 & Rabbit & Genetex \\
\hline AP1G1 & Rabbit & abcam \\
\hline Sortilin & Rabbit & Avia sys. biology \\
\hline
\end{tabular}




\begin{tabular}{|l|l|l|}
\hline ap3m & Rabbit & Avia sys. biology \\
vps41 & Rabbit & DSHB \\
lamp1 & Mouse & \\
Secondary Antibody & & Molecular Probes \\
Anti Rabbit alexa 488 or 594 & & Molecular Probes \\
Anti Mouse alexa 488 or 594 & & \\
\hline
\end{tabular}

For staining the oocytes with different dyes, the oocytes were fixed and blocked same way as described for immunostaining and incubated with required amount of dye in blocking solution under dark. After that, the oocytes were washed and used for imaging.

Table 5. Dyes used in this study

\begin{tabular}{|c|c|c|}
\hline Dyes used & Company & Purpose \\
\hline Cholera Toxin subunit B alexa 594 & Mol. Probes & Lipid raft \\
\hline Dil LDL & Mol. Probes & Trafficking assay In vivo \\
\hline FM4-64 FX Fixable (F34653) & Mol. Probes & Membrane labeling \\
\hline Pepstatin A, BODIPY FL conjugate & Mol. Probes & Cat D labelling \\
\hline Transferrin Alexa 594 & Mol. Probes & Trafficking assay in vivo \\
\hline Mito tracker FM green & Mol. Probes & Mitochondria dye \\
\hline Lysotracker DND Red & Mol. Probes & Lysosome dye \\
\hline NBD-PZ- Lysotracker green & TCI Europe & Lysosome dye \\
\hline MPA texas Red & EY Lab INC & Cortical granule cargo \\
\hline BSA texas Red & Mol. Probes & Endocytosis assay \\
\hline Bungaratoxin alexa 594 & Mol. Probes & AchR binding \\
\hline MPA lectin alexa 488 & Mol. Probes & Cortical granule cargo \\
\hline Jc-1 & Mol. Probes & Mitochondrial activity \\
\hline Firefly latern extract & Sigma & ATP assay \\
\hline rhodamine $2 \mathrm{AM}$ & Mol. Probes & $\mathrm{Ca} 2+$ binding \\
\hline ER tracker green & Mol. Probes & ER dye \\
\hline TMRE & Mol. Probes & Mito. membrane potential \\
\hline Ros detector DHR 123 & Mol. Probes & ROS dye \\
\hline Cholesterol alexa 594 & Mol. Probes & Cholesterol dye \\
\hline
\end{tabular}




\subsection{Motor neuron staining of zebrafish larvae}

\subsubsection{Genotyping of fish}

The 3 dpf fish were selected and the ones that were unhatched were dechorinated manually with two forceps or the adult fish were selected for genotyping. They were anesthetized in a tricaine (3-amino benzoic acid ethyl ester also called ethyl 3aminobenzoate) solution (see Materials and methods, Buffers). When the fish were asleep (when they stopped oving) their heads were cut off with a scalpel, leaving the remaining yolk sac intact. The heads were placed in a 96-well PCR plate and the body in the corresponding well of a 96-well ELISA plate with fixative solution MEMFA (see Materials and methods, Buffers) and leave them at $4^{\circ} \mathrm{C}$ until the staining. The PCR plate with the heads is used for genotyping.

DNA isolation: we lyse the heads with $200 \mu \mathrm{g} / \mathrm{ml}$ of proteinase $\mathrm{K}$ in lysis buffer (see Matherials and methods, Buffers) and 45 minutes at $55^{\circ} \mathrm{C}$. Then we inactivate the proteinase $\mathrm{K}$ for 5 minutes at $95^{\circ} \mathrm{C}$. PCR reaction: the PCR plate is centrifuged shortly to pellet the debris. For the PCR reaction, two different pairs of primers are needed (21403 and 25580). Each pair amplifies a marker genetically linked to soufflé mutation. Each marker amplifies two bands when the genotype is heterozygous and one if it is homozygous. Prepare PCR reactions in PCR buffer (see Materials and methods, Buffers): $200 \mu \mathrm{M}$ dNTPs, $400 \mathrm{nM}$ forward-reverse primer mixture, $40 \mathrm{U} / \mathrm{ml}$ homemade Taq polymerase. Add $10 \mu \mathrm{l}$ to $1 \mu \mathrm{l}$ of DNA. Set PCR reaction (table 2). Add $1 \mu \mathrm{l}$ of loading dye to each sample. The PCR reactions were run and loaded in 3\% agarose (50\% Metaphor agarose and 50\% regular agarose) in TBE buffer at 300 volts for approximately 4 hours. Images were taken in a trans illuminator (Bio Rad).

\section{Table 6. Suf genotyping primers}

\begin{tabular}{|l|l|}
\hline Primers & Sequence \\
\hline Z 21403 Forward & TTGCAATGTTGCATCAGGAT \\
\hline Z 21403 Reverse & TTTGGGGAGAAATGGAGATG \\
\hline Z 25580 Forward & TGACTCTGGGCAACAACTGT \\
\hline Z 25580 Reverse & TGGAAACCTATGGAATGGCT \\
\hline
\end{tabular}


Table 7. PCR reaction setup for genotyping suf

\begin{tabular}{|l|l|}
\hline Substance & Volume $[\boldsymbol{\mu l}]$ \\
\hline $\mathbf{H}_{2} \mathrm{O}$ & 7.8 \\
\hline 10x PCR buffer & 1.0 \\
\hline dNTP 5mM & 0.4 \\
\hline primers fw+bw 10 $\boldsymbol{\mu M}$ & 0.4 \\
\hline home made Taq & 0.4 \\
\hline DNA & 1.0 \\
\hline
\end{tabular}

Table 8. PCR program for suf genotyping

\begin{tabular}{|c|c|c|c|}
\hline Steps & $\begin{array}{c}\text { Temp. } \\
{\left[{ }^{\circ} \mathrm{C}\right]}\end{array}$ & $\begin{array}{c}\text { Time } \\
{[\mathrm{min} / \mathrm{sec}]}\end{array}$ & Cycles \\
\hline $\begin{array}{c}\text { Initial } \\
\text { Denaturation }\end{array}$ & 95 & $3 \mathrm{~min}$ & \\
\cline { 1 - 3 } Denaturation & 95 & $15 \mathrm{sec}$ & \multirow{2}{*}{39} \\
\hline Annealing & 55 & $45 \mathrm{sec}$ & \\
\hline Extension & 72 & $1 \mathrm{~min}$ & \\
\hline $\begin{array}{c}\text { Final } \\
\text { Extension }\end{array}$ & 72 & $3 \mathrm{~min}$ & \\
\hline Pause & 12 & pause & \\
\hline
\end{tabular}

\subsubsection{Motor neuron staining}

They are already fixed in MEMFA, and stored at $4^{\circ} \mathrm{C}$. Block for 1 hour at RT. Add the primary antibodies in blocking solution and leave overnight at $4^{\circ} \mathrm{C}$. Motor neuron antibodies (mouse IgG): zn-1-s (30 $\mu \mathrm{g} / \mathrm{ml}, 1: 200)$, znp-1 (41 $\mu \mathrm{g} / \mathrm{ml}, 1: 200)$, zn-8-s (57 $\mu \mathrm{g} / \mathrm{ml}, 1: 200)$, sv2-s (50 $\mu \mathrm{g} / \mathrm{ml}, 1: 100), 39.4 \mathrm{D} 5$-s (Islet1, $44 \mu \mathrm{g} / \mathrm{ml}, 1: 200)$, F59-s (25 $\mu \mathrm{g} / \mathrm{ml}, 1: 10)$. Wash with PBT for 90 minutes at room temperature with rocking. For those with soufflé staining. Add soufflé antibody (rabbit IgG) 1:200 for 2 hours at room temperature in blocking solution.Wash with PBT for 1 hour at RT. Wash samples twice with PBT for 5' at RT. Add the secondary antibodies 1:200 in blocking solution: goat antimouse conjugated with AlexaFluor $488(2 \mathrm{mg} / \mathrm{ml}$, Molecular Probes) and/or goat anti-rabbit conjugated with AlexaFluor 594 (2mg/ml, Molecular Probes). Leave overnight at $4^{\circ} \mathrm{C}$. Wash 90 minutes in PBT at RT. For the samples stained with $\alpha$-bungarotoxin (conjugated 
with AlexaFluor 594, Invitrogen, Molecular Probes $1 \mathrm{mg} / \mathrm{ml}$ ): add to a final concentration of $10 \mu \mathrm{g} / \mathrm{ml} 45$ minutes at room temperature. Wash with PBT for 15 minutes at RT. Wash with PBT for 15 minutes at RT. Dehydrate and store following the same procedure as for the oocyte immunostaining.

\subsection{Cell culture techniques}

\subsubsection{Propagation of cell lines}

Cells were cultured in DMEM medium (Biochrom) supplemented with $10 \%$ fetal calf serum (FCS), 100 units $/ \mu 1$ penicillin and $100 \mu \mathrm{g} / \mathrm{ml}$ streptomycin (full DMEM). Cell lines were maintained at $37^{\circ} \mathrm{C}, 95 \%$ humidity, $5 \% \mathrm{CO}_{2}$.

\subsubsection{Subculturing of cell lines}

The cells in $75 \mathrm{~cm}^{2}$ flask were rinsed with 1x PBS and incubated with $2-3 \mathrm{ml}$ of $0.25 \%(\mathrm{w} / \mathrm{v})$ trypsin $10-20 \mathrm{~min}$ at $37^{\circ} \mathrm{C}$ until the cell layer was dispersed. The reaction was stopped with $5 \mathrm{ml}$ of DMEM medium. The appropriate number of cells was transferred into a fresh $75 \mathrm{~cm}^{2}$ flask containing $15 \mathrm{ml}$ of fresh full DMEM medium.

\subsubsection{Cryo-preservation of cells}

In order to store cell lines for a longer period of time cells were trypsinized and resuspended in pre-warmed DMEM medium. The cell-suspension was transferred into a 50 $\mathrm{ml}$ falcon tube and centrifuged for $5 \mathrm{~min}$ at $1000 \mathrm{rcf}$. The supernatant was removed and the cells were re-suspended in cold FCS medium containing 10\% DMSO. This suspension was transferred into cryo vials (Greiner Labortechnik), put on ice for $15 \mathrm{~min}$ and then stored at $80{ }^{\circ} \mathrm{C}$. After one day cryo-cultures were transferred to liquid nitrogen $\left(-196^{\circ} \mathrm{C}\right)$.

\subsubsection{Thawing of cryo-preserved cells}

$500 \mu \mathrm{l}$ of pre-warmed DMEM medium were added to the frozen cells in the cryo vials. The solution was pipetted up and down and then transferred into $50 \mathrm{ml}$ falcon tube filled with $10 \mathrm{ml}$ of fresh DMEM. These steps were repeated until all cells were thawed. Afterwards, the cells were centrifuged for $5 \mathrm{~min}$ at $1000 \mathrm{rcf}$, the supernatant was removed, the cell pellet was re-suspended in fresh culture medium, and finally transferred into a fresh 
$75 \mathrm{~cm}^{2}$ flask containing $10 \mathrm{ml}$ of fresh DMEM medium. Cells were maintained at $37{ }^{\circ} \mathrm{C}$, $95 \%$ humidity, and $5 \% \mathrm{CO}_{2}$.

\subsubsection{Transfection of cells}

HEK293 cells were transfected with Lipofectamine2000 ${ }^{\mathrm{TM}}$ (Invitrogen) according to the manufacturer's instructions. Cells were plated 24 hours before transfection in DMEM containing 10\% FCS but no antibiotics. The amounts of DNA and Lipofectamine $2000^{\mathrm{TM}}$ as well as the seeding density of cells are calculated according to the procedure. DNA and Lipofectamine $2000^{\mathrm{TM}}$ were diluted in OptiMem (Gibco), incubated 5 minutes at room temperature, then DNA and Lipofectamine samples were combined and incubated $15 \mathrm{~min}$ at room temperature. The mixture was applied on the cells for 4 hours, after which the plating medium was changed for fresh full DMEM.

\subsubsection{Fixation of transfected HeLa cells}

48 hours after transfection, the cells were washed two times with 1x PBS and then fixed for $15 \mathrm{~min}$ using 4\% (w/v) paraformaldehyde. Thereafter, cells were washed two times with 1x PBS. Meanwhile DAPI (20mg/ml, Sigma) was diluted (1:1000) with mounting medium (Dako) and one drop of this dilution was put onto a glassslide. Subsequently, the coverslip with the fixed cells (cell side towards the drop) was put onto the drop. For drying of the mounting medium, the cover slip was stored at $4{ }^{\circ} \mathrm{C}$.

\subsubsection{Immunofluorescent staining of cells (Susanne Schlick)}

Cell were grown in $75 \mathrm{~cm} 2$ flasks to $90-100 \%$ confluence, and then transferred to 6well plates (Nunc) with 4 coverslips (Marienfeld or Menzel) each. Cells were grown in 1:1 old filtered medium: fresh medium. Immunostaining was carried out in 24- well plates (Sarstedt). Cells grown on coverslips were washed in warm PBS and then transferred into 24- well plates with warm 4\% PFA. Fixation lasted 20min. Subsequently cells were washed 3 times with PBS for 5min, followed by permeabilization in PTX (PBS with 0,5\% Triton) for $5 \mathrm{~min}$ and then blocking for 30min in PTX+ 0,5\% BSA. Cells were then incubated overnight at $+4^{\circ} \mathrm{C}$ with the primary antibody in blocking solution or for controls only in blocking solution. The following day they were washed 3 times for $5 \mathrm{~min}$ in PBS and then incubated with the secondary antibody for 1 hour in fresh blocking solution. Following incubation cells were washed 3 times with PBS followed by staining with DAPI $(1 \mu \mathrm{g} / \mathrm{ml})$ 
in blocking solution. Cells were kept in Millipore water afterwards and then mounted onto objective plates with either Mowiol or AquaPolymount (Polysciences). Coverslips were sealed with nail polish. Slides were stored in the dark at $+4^{\circ} \mathrm{C}$.

\subsubsection{In-vivo trafficking assay}

Trafficking assay, adopted after Rapaport et al., 2010.

Following proteins were used: Transferrin from human serum conjugated with Alexa 594 at a working concentration of $25 \mu \mathrm{g} / \mathrm{ml}$, cholera toxin subunit b conjugated with Alexa 594 at a working concentration of $0,5 \mu \mathrm{g} / \mathrm{ml}$, Lectin DHA- L conjugated with Alexa 488 at a working concentration of $10 \mu \mathrm{g} / \mathrm{ml}$ and low- density lipoprotein (LDL) (human plasma), DIL complex, $10 \mu \mathrm{g} / \mathrm{ml}$ (all Invitrogen). Dilution of working solutions into serum- free RPMI- 1640 (Biochrom AG), containing 20mM HEPES and 0, 1\% BSA. Cells were serum- starved in RPMI- 1640 with 20mM HEPES and 0,1\% BSA for $30 \mathrm{~min}$ at $37^{\circ} \mathrm{C}, 5 \%$ $\mathrm{CO} 2$. The protein suspension was added and cells were incubated for $1 \mathrm{~h}$ at $+37^{\circ} \mathrm{C}, 5 \%$ $\mathrm{CO} 2$. Cells were then rapidly chilled on ice, washed 3 times $5 \mathrm{~min}$ with cold PBS (kept on ice at all times) and then fixed in 4\% PFA for 20min. After a quick wash in cold PBS, cells were mounted with Mowiol and coverslips were sealed with nail polish. Slides were stored in the dark at $+4^{\circ} \mathrm{C}$.

\subsection{Protein techniques}

\subsubsection{Suf antibody production}

Antibody against suf was made using two selected peptides covering one in $\mathrm{N}$ Terminal and one in C-terminal. The antibody was produced in two rabbits by a company Biogene, Germany. The serum was column purified and tested in ELISA for their specific binding to the protein or peptide.

Two selected peptides are

\section{7-200:C-TEQVKVPAKDRNRE}

\section{2-1025:C-LNKTSTNKGMSKTD}

The antibody was checked in western blot for their binding using extract from wt and suf mutant consract overexpressed cell. 


\subsubsection{Harvest of cells for Western Blotting}

Cell lysate preparation was done on ice. Cells adherent to the surface of the plate were scraped off into the medium and transferred into an Eppendorf tube. Cells were pelleted down by centrifuging at $4000 \mathrm{rpm}$ for $3 \mathrm{~min}$ at $4^{\circ} \mathrm{C}$. Media was removed and $1 \mathrm{X}$ PBS was added for washing the cells. Cells were resuspended in appropriate amount of lysis buffer; depending on the pellet size, for a 6-well plate, 100-120 $\mu 1$ while for a 12-well plate, 50-60 $\mu 1$ of lysis buffer was used. Cells were briefly vortexed and kept on shaking at $4^{\circ} \mathrm{C}$ for $20 \mathrm{~min}$ for efficient lysis of the cells. Cell lysate was then centrifuged at 13,000 rpm for 10min to let DNA settle down.

Bicinchoninic acid assay (BCA assay) kit was used to normalize the concentration of proteins. In this assay, total concentration of protein is exhibited by a color change of sample solution from green to purple in proportion to protein concentration, which can then be measured by colorimetric techniques. According to user's manual, BCA reagents were mixed in the ratio $\mathrm{A}: \mathrm{B}=98: 2$ and $5 \mu \mathrm{l}$ of protein were added to this mixture, incubated at $37^{\circ} \mathrm{C}$ for half an hour. Using Nanodrop spectrophotometer, a standard curve was prepared with different dilutions of BSA (provided with the kit); concentration of proteins was then measured using this standard curve. To the normalized amount of protein, 6X Laemmli buffers was added to the final concentration of $1 \mathrm{X}$ and samples were boiled at $95^{\circ} \mathrm{C}$ to reduce the disulfide bonds and denature the proteins.

\subsubsection{Protein electrophoresis under the denaturating conditions (SDS-PAGE)}

The proteins were separated by SDS polyacrylamid gel electrophoresis (Laemmli.1970). Gels of the different acrylamid percentages were used for the analysis of the proteins with distinct molecular weights according to the Table 14.

Table 9. The sizes of separated proteins and the percentages of the correspondent acrylamid gels

\begin{tabular}{|c|c|}
\hline Protein size, $\mathrm{kDa}$ & \% of acrylamide \\
\hline $36-205$ & $5 \%$ \\
\hline $24-205$ & $7.5 \%$ \\
\hline $14-205$ & $10 \%$ \\
\hline $14-66$ & $12.5 \%$ \\
\hline $10-45$ & $15 \%$ \\
\hline
\end{tabular}


The protein samples were diluted 1:5 with 6x Laemmli loading buffer and boiled for 5 min at $95^{\circ} \mathrm{C}$, then applied on the gel. The gel running was performed in the BioRad ${ }^{\mathrm{TM}}$ gel chambers in 1x Laemmli running buffer. At first the voltage of $70 \mathrm{~V}$ was applied, and once the bromphenol-blue front reaches the separating gel, the voltage was raised to $120 \mathrm{~V}$.

\subsubsection{Western Blotting}

The technique (also known as Protein Immunoblotting) allows detection of specific proteins in a cell lysate. The method was introduced by Towbin et. al. (1979) (Harry Towbin.1979) and is now a routine technique for protein analysis. The proteins separated by electrophoresis are transferred to a nitrocellulose or polyvinylidene difluoride (PVDF) membrane. Membrane is then incubated with an antibody (called as primary antibody) against the epitope of a specific protein, followed by addition of another antibody (called as secondary antibody) which can bind to the species-specific region of the primary antibody and is conjugated to an enzyme like Horseradish peroxidase. The enzyme can convert its substrate into a product that produces luminescence, the light output is directly proportional to the amount of protein and can be captured by using film, a CCD camera or a phosphorimager designed for chemiluminescent detection.

Once proteins were separated by SDS-PAGE, a sandwich of gel and membrane was prepared for electroblotting of proteins from gel to membrane. Transfer was performed at constant voltage of $100 \mathrm{~V}$ for $120 \mathrm{~min}$ (for the transfer of big proteins, PVDF membrane was used and transfer was done at constant voltage of $40 \mathrm{~V}$ for $24 \mathrm{~h}$ ). After transfer was finished, membrane was stained with Ponceau S to check whether transfer was uniform and proteins were equally loaded. For blocking the unspecific sites on the membrane, where antibodies can bind, blocking buffer was added to the membrane for $45 \mathrm{~min}$. It was followed by overnight incubation with appropriate dilution of primary antibody at $4^{\circ} \mathrm{C}$, washing of the primary antibody with washing buffer (PBST or TBST) and addition of secondary antibody (1:10,000 dilution; for both primary and secondary antibody blocking buffer was used for making dilutions) for $1 \mathrm{~h}$ at room temperature. Membrane was then once washed with blocking buffer, followed by washing buffer. For visualizing the amount of protein, suitable amount of substrate solution (Immobilon Western HRP Substrate Peroxide Solution) was applied and luminescence was detected using a Chemocam HR 16 3200 imager. For weak signals, the more sensitive substrate solution SuperSignal West Femto Maximum Sensitivity Substrate was used. 


\subsection{Statistics}

In all experiments, error bars indicate the standard deviation of the average (at least three independent experiments). The statistical significance (p-value) of two groups of values was calculated using a two-tailed, two-sample unequal variance t-test calculated in MS-Excel or www.graphpad.com. The graph was drwn using MS-Excel.

\subsection{Bio-informatic analysis}

The bioinformatics prediction were done for Suf and VPS41 using http://toolkit.tuebingen.mpg.de/. HHpred - Homology detection \& structure prediction by HMM-HMM comparison was used to predict the protein domain in both proteins. 


\section{Results}

\subsection{Soufflé localizes to vesicles}

Soufflé mutants seed opaque egg instead of transparent eggs and fail to develop further. Suf protein contains the FYVE domain, which binds to PI3P on endosomal membranes, and the morphological phenotype of the mutant egg suggests that the protein plays a vital role in vesicle trafficking processes. Therefore, to find if the soufflé protein localizes to vesicles, I generated an antibody against two peptides covering $\mathrm{N}$-terminal and C-terminal region of zebrafish Suf protein and used it for immunization of two rabbits. The purified antibody has been ELISA tested for the interaction with the specific peptide. Immuno-staining of wild-type oocytes for Suf proteins confirmed that it indeed localizes to vesicles as predicted. Surprisingly, the mutant oocyte also showed similar localization pattern like wild type which suggests that the suf mutant protein still localizes with its intact FYVE domain properly (Fig.1).This results show that the Suf protein localizes to vesicle in both wildtype and mutant signifying the importance of the deleted region (SUF domain) in Suf protein for its role in vesicle trafficking during oogenesis.
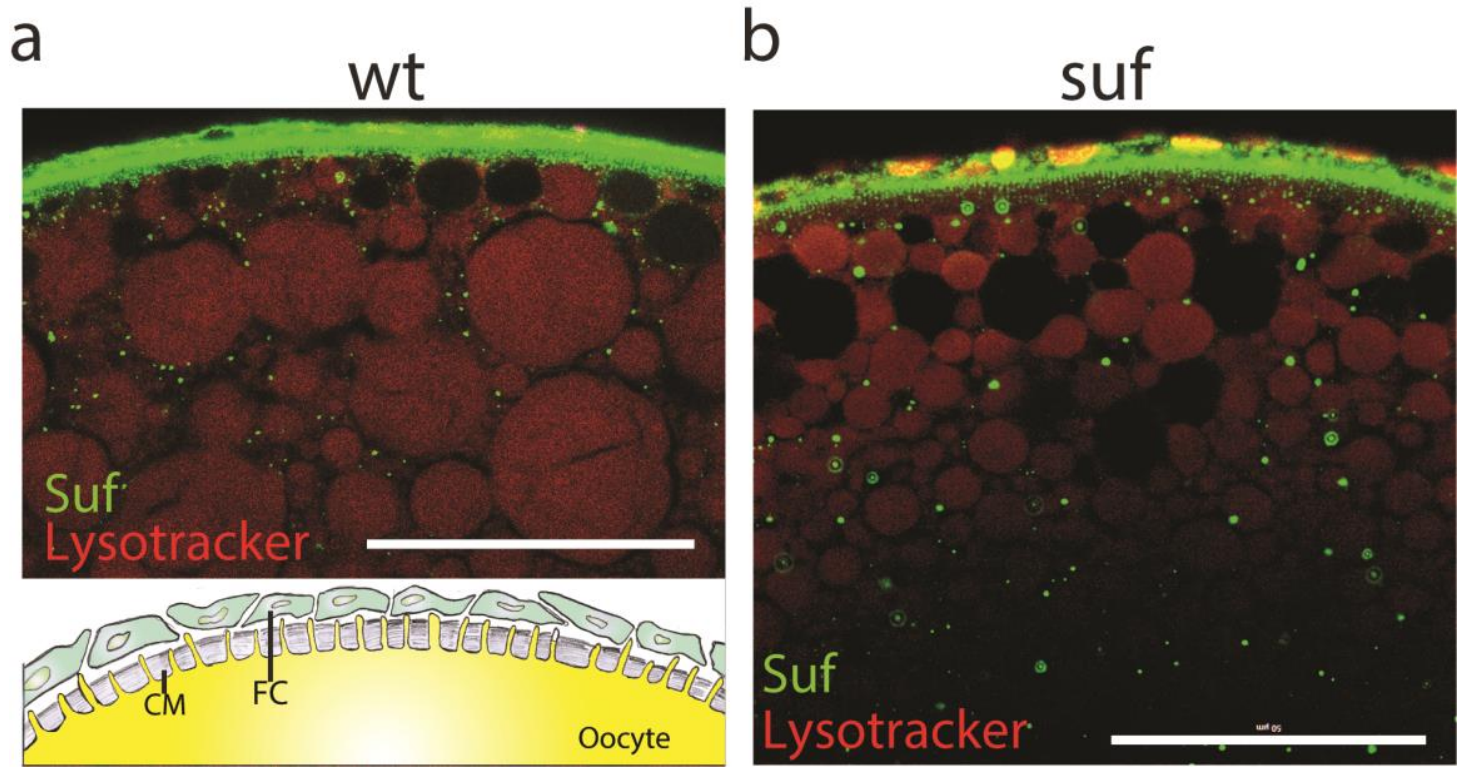

Figure 16. Suf protein localizes to vesicles.

a. Immuno-staining for suf protein in wild-type oocytes with wild-type oocyte structure in cartoon below. Suf in green and yolk globule/Lysosomes in red. b. Immuno-staining for suf and lysosome in suf mutant oocytes. CM- Chorion membrane, FC- Follicle cells, Scale-50 $\mu \mathrm{m}$. 


\subsection{Soufflé mutation produces truncated Suf protein}

Mutation in Soufflé gene in human causes progressive motor neuron degeneration while we did not see this defect in the adult fish possessing the zebrafish soufflé mutation. This raises the question that suf might have tissue specific variants and functions or the mutant protein still has a reduced function, which is sufficient to takeover zygotic function but fails during oogenesis.
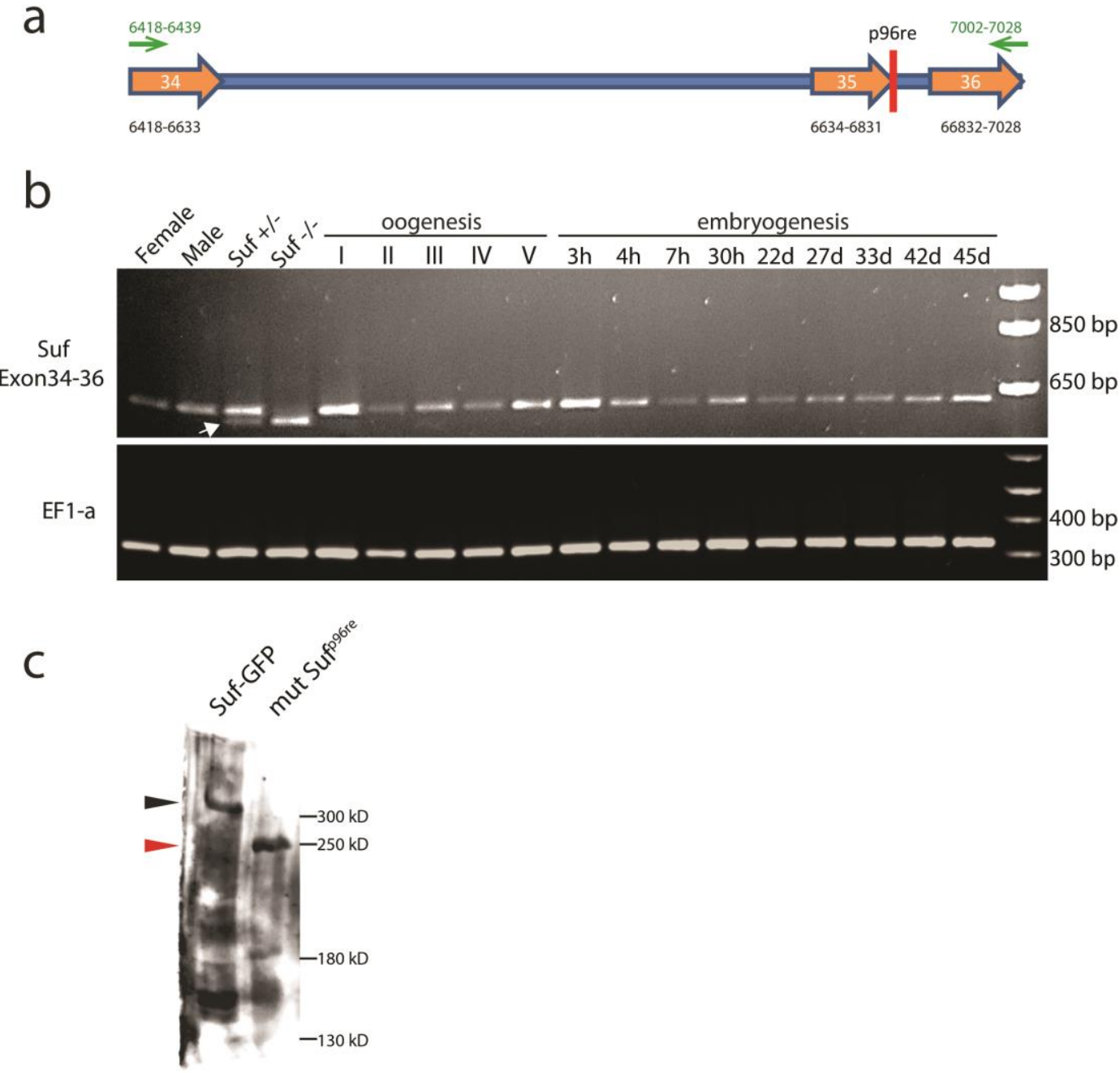

\section{Figure 17. Suf p96re mutation creates truncated suf protein.}

a. Cartoon showing the amplified region of exons 34, 35 and 36 in suf gene with corresponding introns, red line next to exon 35 shows the p96re mutation. b. Agarose gel picture showing the PCR amplified product-covering exon 34,35 and 36 . The expected bad size with exon 35 is $650 \mathrm{bp}$, without exon 35 is $413 \mathrm{bp}$. All the stages shows $650 \mathrm{bp}$ band in all stages while heterozygous and homozygous mutant showing one additional shorter transcript due to the mutation, arrowhead shows the mutant version of the suf transcript. EF1 $\alpha$ used as PCR and loading control. c. Western blot for suf protein in tissue culture cell lysate using anti-suf antibody. Suf-GFP shows the wt band 
at above $300 \mathrm{KDa}$ (Black arrowhead) while mutant version shows the truncated version at $250 \mathrm{KDa}$ (Red arrowhead).

To examine whether an alternatively spliced Suf/Spastizin mRNA hides a potential zygotic mutant phenotype in other tissues or in males, we analyzed the expression of exon 35 carrying the p96re mutation during zebrafish embryogenesis and oogenesis. But, we did not observe a shorter transcript lacking exon 35, which would generate a 413 bp product. However, in hetero- or homozygotes we detected the predicted 25 bp shorter transcript consistent with the mutation in splice donor site (Fig. 2a \& b). Since Suf/Spastizin expression at the mRNA level was not completely eliminated, the residual protein might have sufficient activity to compensate for Suf/Spastizin requirement in somatic cells. Although the quantitative RT-PCR suggested that the mRNA is degraded or reduced in the mutant, the immuno-staining showed Suf protein localization in the mutant oocyte. To check if the suf mutant protein also expresses and the antibody specifically detects the zebrafish Suf protein, I overexpressed the zebrafish Suf gene in tissue culture cells and observed the suf protein in the western blot. To differentiate the wild-type protein from mutant version, I fused GFP N-terminally with Suf, which increases the chance of differentiating the protein size in the western blot. The suf antibody detected wild-type full length and truncated Suf protein in the mutant version while non-transfected cells did not show any band (Fig.2c).These results show that the mutated exon is present during all stages of development in all tissues and the mutant transcript still produces sufficient amount of truncated protein which localizes to membrane and vesicles, might still have a reduced function. Notably, the $p 96 r e$ allele is almost identical to one of the HSP mutations in human (Hanein et al.,2008) and probably encodes a hypo morph, whose reduced function becomes apparent in the oocyte of zebrafish with its high vesicle trafficking activity.

\subsection{Suf mutants oocytes show endo-lysosomal defect}

Suf mutant's opaque phenotype suggested that there is a defect in yolk degradation in the endo-lysosomal pathway. The preliminary studies from the lab using electron microscopy showed that the suf mutants accumulate smaller endosomes compared to endosomes in the wildtype. Also in tissue culture cells, the FYVE domain of Suf/Spastizin interacts with the endosomal lipid PI3P indicating a role in endosomal trafficking (Sagona et al.,2010). Moreover, in human and mouse cells Spastizin binds to the novel AP5 
complex regulating endosomal transport (Hirst et al.,2011; Hirst et al.,2013a; Hirst et al.,2013b; Khundadze et al.,2013).

\subsubsection{Suf mutant oocytes accumulates Rab11 positive vesicle}

To examine genetically in zebrafish oocytes, whether Suf/Spastizin is involved in endocytosis during oogenesis and at which step of the endocytotic pathway is affected in suf mutant, I used live imaging analysis with the oocyte injected with fluorescently labelled marker proteins (Rab5-CFP, Rab7-CFP and Rab11-CFP). So that I can also track the vesicle transport in-vivo. But unfortunately, the results suggested that the auto-fluorescence from the yolk protein of oocyte gave a high background that hindered proper visualization of the vesicle (Fig.3a). Hence, I decided to use immuno-staining for the marker proteins and compared endosomal compartments between wild-type and mutant oocytes (Fig.3b). The gross morphology of oocyte vesicles showed no difference in early endosomes (Rab5) or late endosomes (Rab7) (Fig. 4). In contrast, Rab11b-positive recycling endosomes showed a remarkable transformation of their tubular shape in wt to patches accumulating below the nuclei of the surrounding follicle cells in mutant oocytes (Fig.4).

a
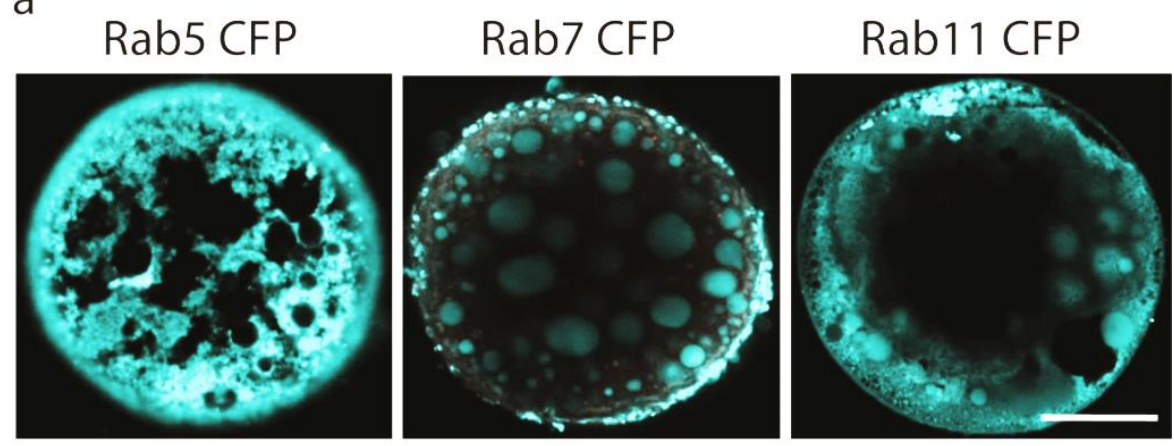

b

Rab5

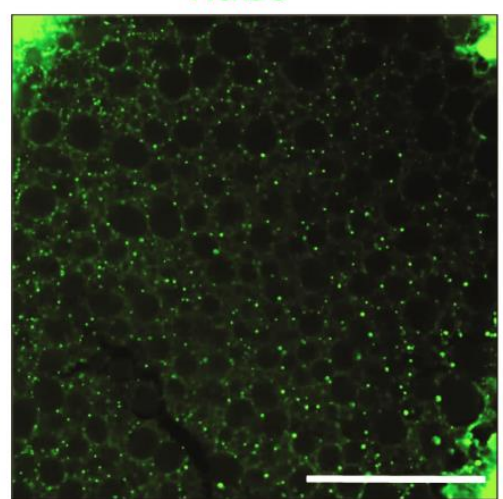

Rab11

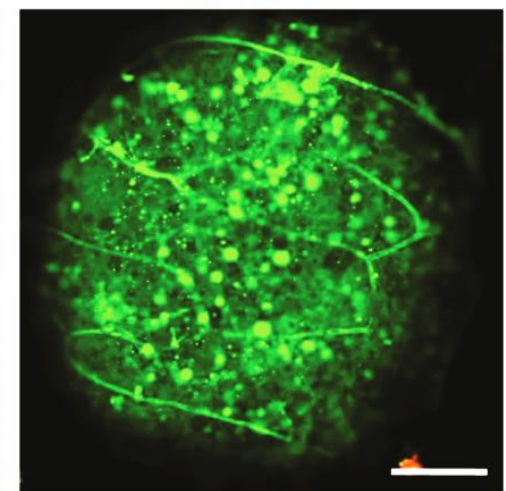

Figure 18. Immuno-staining of oocytes. 
a. Confocal picture of fluorescent marker protein injected oocytes. Rab5, Rab7 and Rab11 in Cyan. Big yolk globules also give auto fluorescence. b. Antibody staining for the endosomal marker proteins in fixed oocytes. Rab5 and Rab11 in green. The vesicular structure of early endosome (Rab5) and the tubular structure of recycling endosome (Rab11) is also visible clearly. Sclae-50 $\mu \mathrm{m}$.

To quantify the defect in Suf/Spastizin mutants, I counted the Rab positive foci in optical sections in deeper layers of the oocyte cytoplasm, where single, small vesicles are separated well and easier to discriminate than the large compartments at the cortex (Fig.5). Rab5 (early endosomes) showed no significant change ( 1.09 fold; $p=0.35$; Fig.5a), whereas Rab7 positive endosomes increased moderately ( 1.78 fold; $p=0.0001$; Fig.5b). However, Rab11 staining increased dramatically (3.82 fold; $p=0.0001$; Fig.5c) confirming the initial observation that Suf/Spastizin controls trafficking of recycling endosomes in zebrafish oocytes, though other endosomal compartments are not affected in suf mutants.

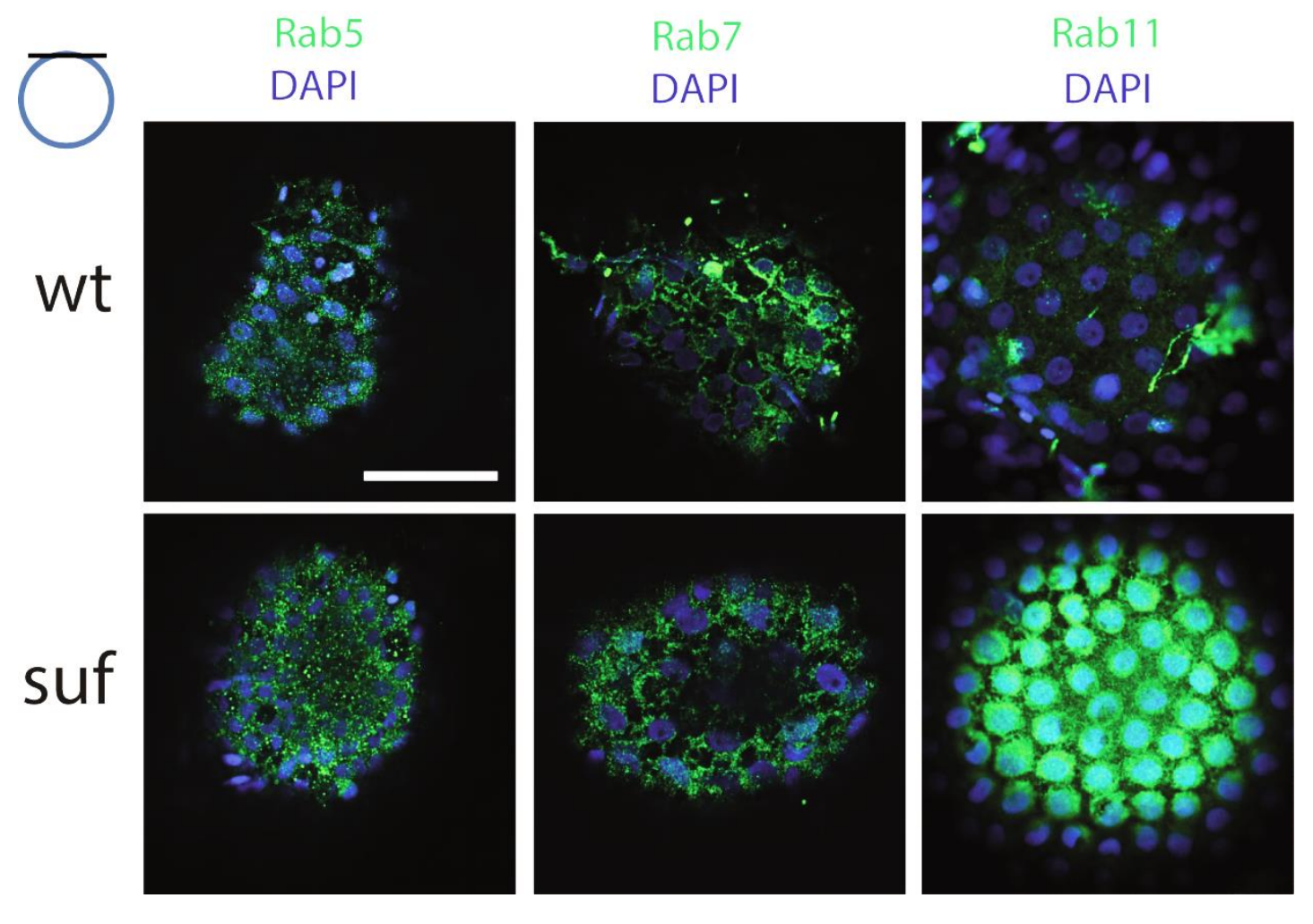

Figure 19. Rab11 accumulates in the cortex of suf mutant oocyte.

Top panel shows staining for rab proteins in wild-type oocytes and bottom panel shows the staining from suf mutant oocytes. Rabs in green and nuclei of the follicle cells in blue. Small cartoon at the left top shows the optical section plane in the confocal microscopy where the image was taken. Scale- $50 \mu \mathrm{m}$. 


\subsubsection{Suf mutant oocyte accumulate immature small lysosomes}

The accumulation of rab11 positive recycling endosome does not explain why the yolk protein is not degraded. The yolk proteins reaches the lysosome during oogenesis and they are cleaved by different Cathepsin proteases which makes the opaque egg into a transparent one. To check if the lysosomes are formed properly in the suf mutant, the oocytes are stained with lyso-tracker red and the results showed that the suf mutant accumulates smaller fragmented yolk globules or lysosomes. This result confirms that the smaller endosome accumulation detected in electron microscopy are immature lysosomes, consistent with previous reports in cell culture and mouse mutant studies. This defect in the lysosome explains that preventing the yolk proteolysis in the egg and causing the opaque cytoplasm phenotype (Fig. 5 \& Fig. 13). 

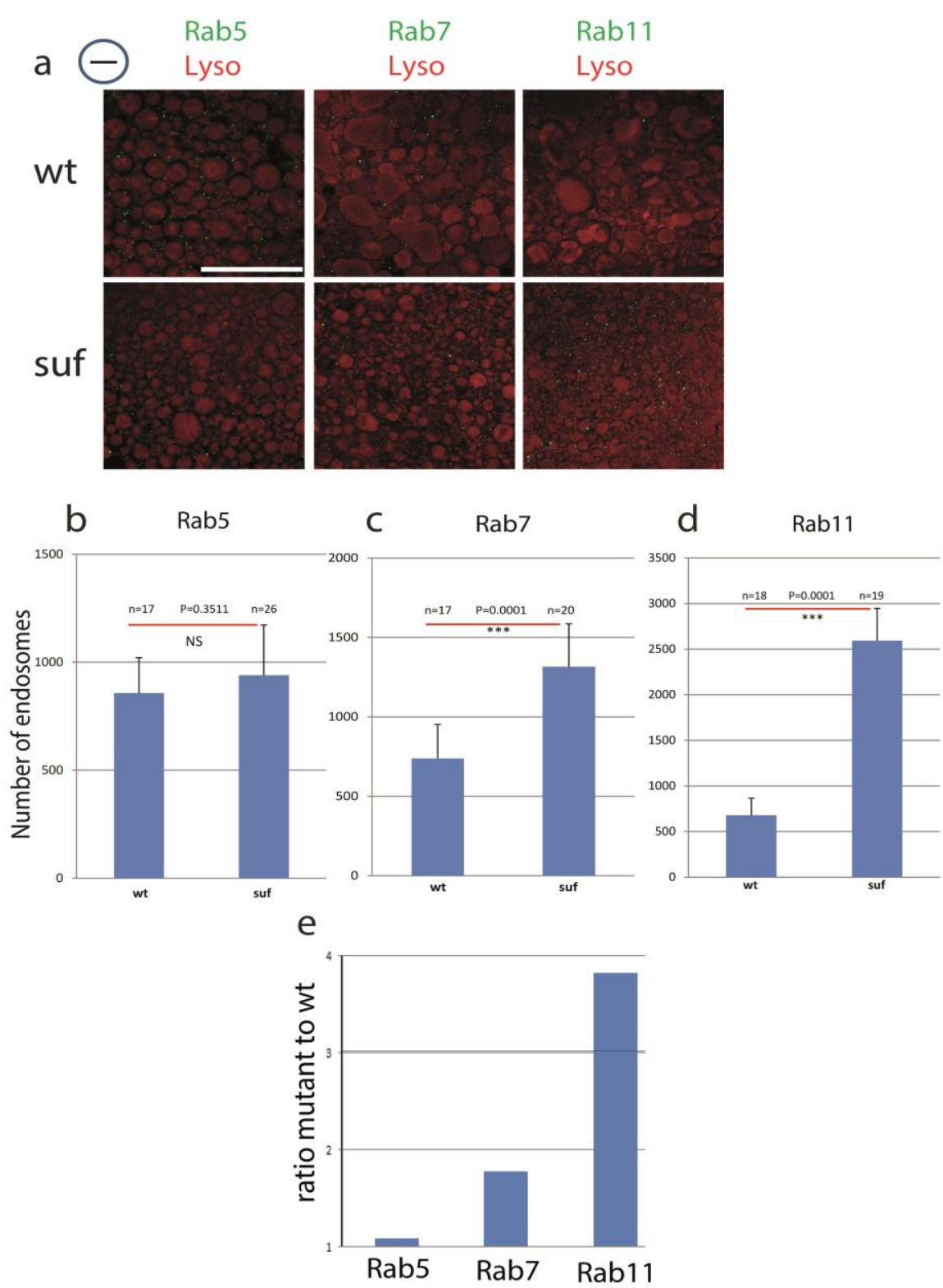

\section{Figure 20. Rab11 and Rab7 accumulate in suf mutant oocytes.}

a. Staining for lysosome in red and rab proteins in green. Top panel from wild-type oocytes and bottom panel from suf mutant oocytes. Cartoon at the left top shows the position of the optical section of the oocyte. Scale- $50 \mu \mathrm{m} . \mathrm{b}, \mathrm{c}$ and d. The bar diagram shows the quantification individual vesicle positive for respective marker proteins Rab5, Rab7 and Rab11 in wildtype and mutant oocytes. In y-axis the mean number of endosomes and NSnon significant, $* * *$ - extremely significant. e. Bar diagram shows the change in the number 
of endosomes in the suf mutant compared to wildtype. Y-axis shows the ratio of mutant to wt.

\subsection{Recycling endosome is not affected in the suf mutant}

To analyze functionally during oogenesis whether the observed accumulation of the compartment-specific Rab proteins reflects a defect in the corresponding transport route as described for tissue culture cells, I established an in-vivo cargo trafficking assay in the zebrafish oocyte.

Transferrin follows the recycling route through recycling endosome (Karin and Mintz.1981; Willingham et al.,1984; Yamashiro et al.,1984). The zebrafish Transferrinreceptor is maternally expressed and the Transferrin recycling assay was previously applied to Xenopus oocytes (El-Jouni et al.,2007; Chen et al.,2013). Notably, contrary to the accumulation of Rab11 vesicles, fluorescent Transferrin did not accumulate in Suf/Spastizin oocytes during different time point analysis (Fig.6). More interestingly, I found partial overlap of Transferrin with Rab11 suggesting that the Rab11b antibody and Transferrin label two different compartments in zebrafish oocytes. The in-vivo analysis indicates that suf mutant does not have a defect in recycling endosomes. 


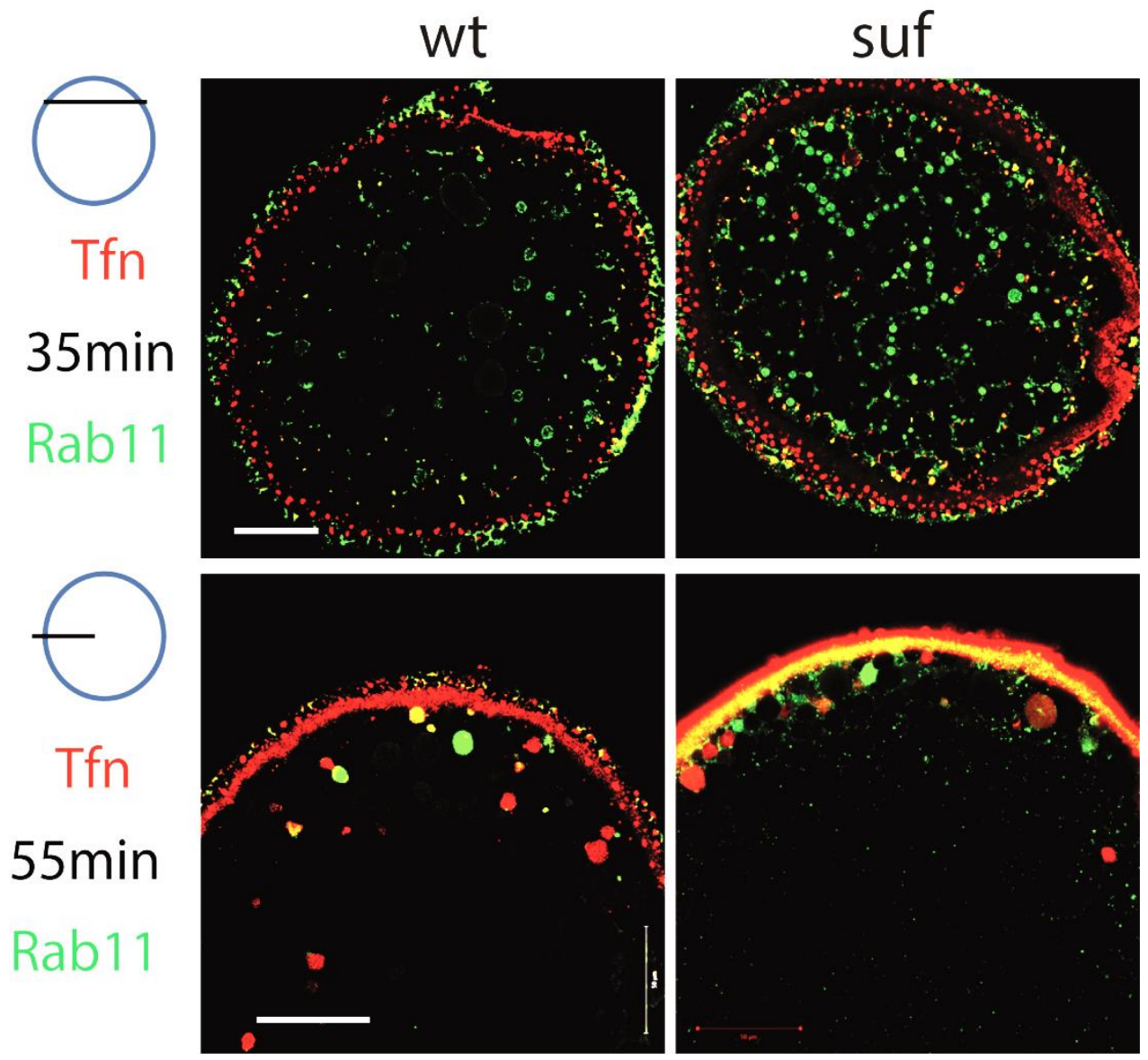

Figure 21. Tfn recycling is not affected in the suf mutant.

The picture shows the confocal image of oocytes loaded with transferrin in Red and Rab11 in green at two different time points. Left panel shows the recycling assay from wild-type oocytes and right side panel shows from suf mutant oocytes. Top two images from oocytes after 35 mins of transferrin recycling and bottom images from after 55 mins of recycling Cartoon shows the optical section plane. Scale- $50 \mu \mathrm{m}$.

\subsection{Suf localizes to Rab5, Rab11 positive endosome and lysosome}

To analyze whether Suf/Spastizin directly controls the trafficking of endosomes or whether the accumulation of Rab11b-positive vesicles is a secondary defect, we investigated Suf/Spastizin and Rab proteins co-localization in the oocyte. Although early endosomes did not show a defect in Suf/Spastizin mutants, we also discovered colocalization of Suf/Spastizin with Rab5 as shown in the mouse (Khundadze et al.,2013), but not with Rab7 (Fig.7a and b). Although Rab11b and Suf/Spastizin mostly overlapped in their localization, some Rab11b vesicles were negative for Suf/Spastizin (Fig.7a and b). Also suf localize to the lysosomes consistent with previous reports (Khundadze et al.,2013). This finding in the zebrafish oocyte indicates that Suf/Spastizin is not involved in 
all processes regulated by Rab11b. In summary, the loss of Suf leads to an accumulation of Rab11b positive vesicles, which in zebrafish oocytes are not involved in Transferrin recycling.

\subsection{Role of Suf on Rab11 positive vesicles}

\subsubsection{Rab11 and Suf localizes to Secretory granule (Cortical Granule)}

Cell culture studies implicate Rab11 endosomes in additional transport processes besides recycling (reviewed in Taguchi.2013)e.g. Rab11 localizes on secretory vesicles of mammalian cells (Urbe et al.,1993). Yeast, tissue culture cells and C. elegans oocytes require Rab11 for exocytosis of secretory vesicles (Benli et al.,1996; Jedd et al.,1997; Chen et al.,1998; Khvotchev et al.,2003; Sato et al.,2008), which are also labeled by Rab11b (Ullrich et al.,1996). The plant cells use Rab11 positive secretory vesicles during cytokinesis in the cell plate (ref). Moreover, in epithelial cells, Rab11a and -b localize to distinct compartments (Lapierre et al.,2003b).In oocytes of many organisms including humans, secretory vesicles are also designated as cortical granules (reviewed in Wessel et al.,2001; Liu.2011). They are very similar to large, dense-core vesicles found in secretory cells in humans e.g. neurons or pancreatic B-cells (Tooze et al.,2001; Meldolesi et al.,2004; Kim et al.,2006).

To determine in fish oocytes, whether Rab11b marks secretory granules, we doublelabeled them with the cortical granule marker MPA-lectin and Rab11 (Becker and Hart.1999). Rab11b co-localized with MPA-lectin identifying the Rab11 vesicles of the zebrafish oocyte as secretory, that is cortical granules (Fig. 8a, a'). Moreover, Suf/Spastizin protein also co-localized with MPA on cortical granules (Fig. 8b, b'). Since not all MPAvesicles were positive for Rab11b or Suf, we hypothesized that co-labeling is only observed at the vesicle surface. In contrast, if the optical section is more central in the vesicle, the green Rab11b or Suf-signal was encompassing the luminal MPA-positive cargo. Since Suf was highly enriched in the cortex and hence, co-localization might be caused by protein abundance, we analyzed optical sections in deeper layers of the oocyte with less Suf signal (Fig. 8c-e). Indeed, Rab11b and Suf mostly localized outside the granule-lumen (Fig. 8c, d). Higher magnifications showed a vesicle with surface staining (Fig. 8e upper row) and another example with an extra luminal micro domain (Fig. 8e lower row), which was observed for Rab11b and Suf. Taken together, the co-localization 
with Rab11b and MPA-lectin support a role of Suf/Spastizin in the formation of cortical granules during zebrafish oogenesis. 
Results
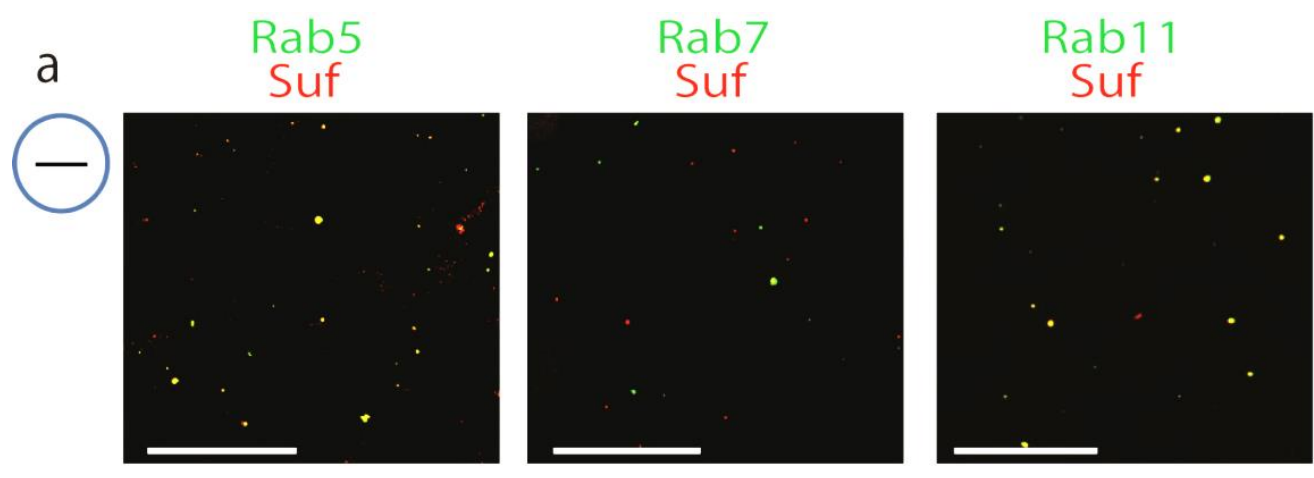

b

Rab5
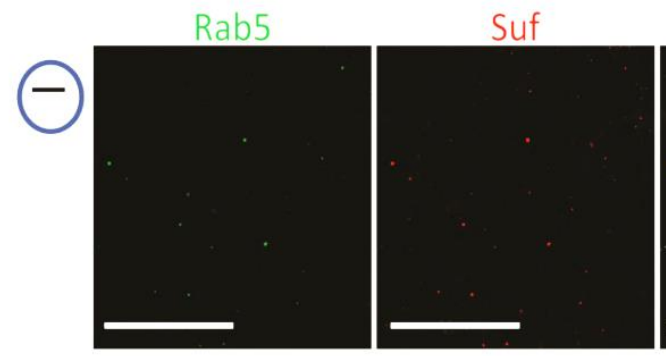

merge

Rab7

Suf
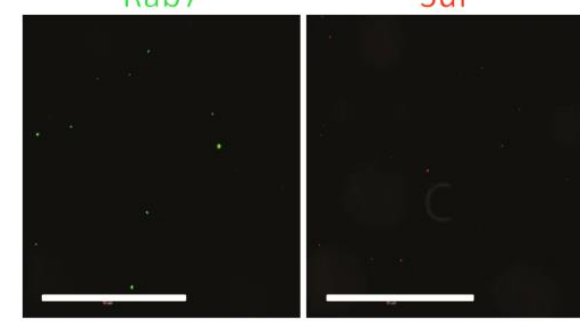

merge
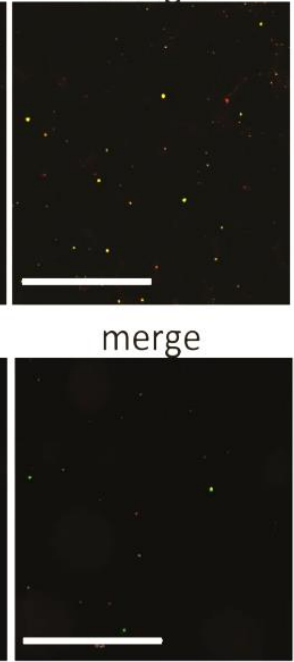

Rab11

Suf

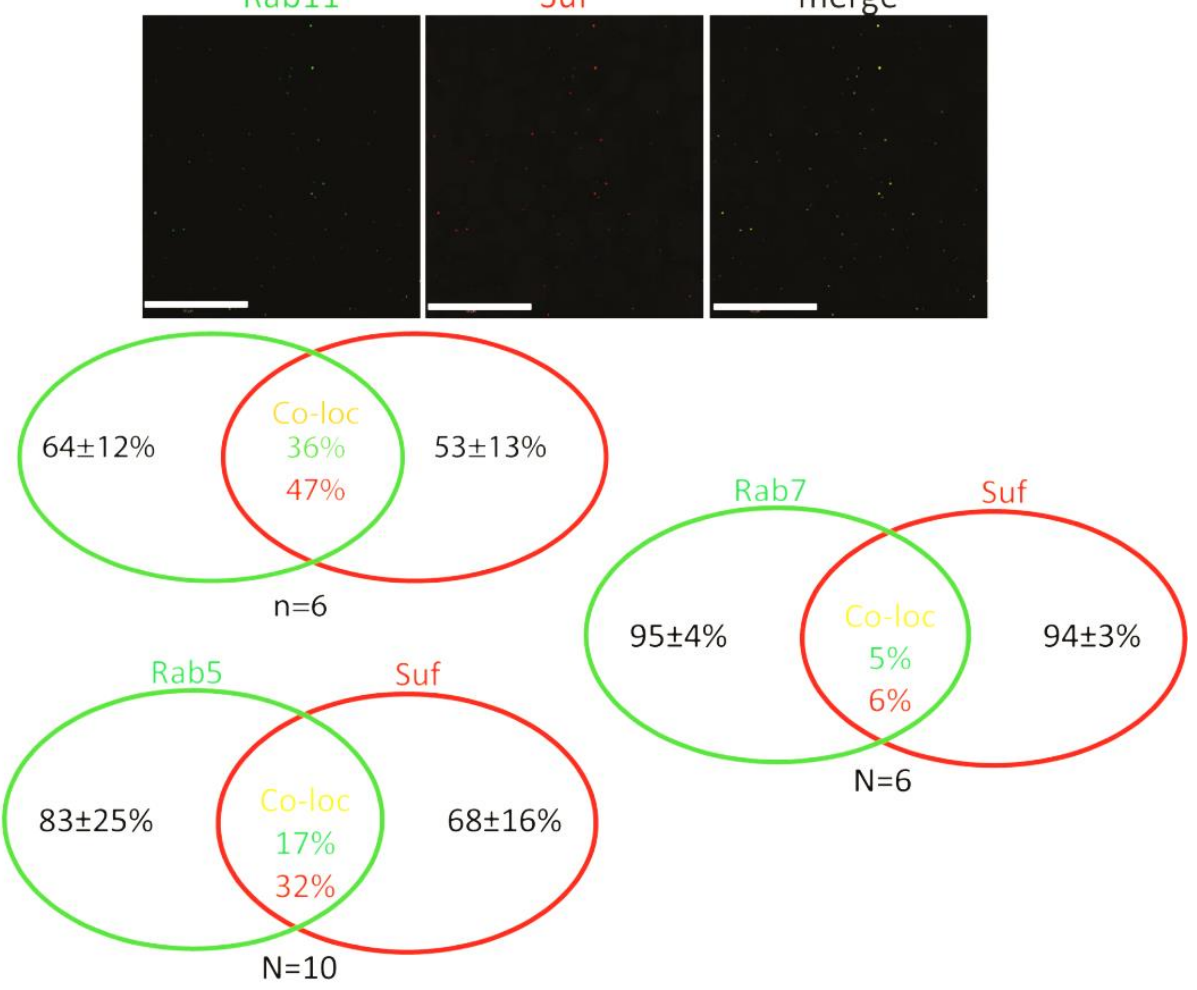


Figure 22. Suf colocalize with Rab5, Rab11 and lysosome.

a. Double staining of suf in red with endosomal markers in green. b. Separated individual channel of two proteins and merged picture. Scale $-50 \mu \mathrm{m}$. The venn diagram shows the amount co-localization between suf and other rab proteins. Cartoon shows the optical plane.

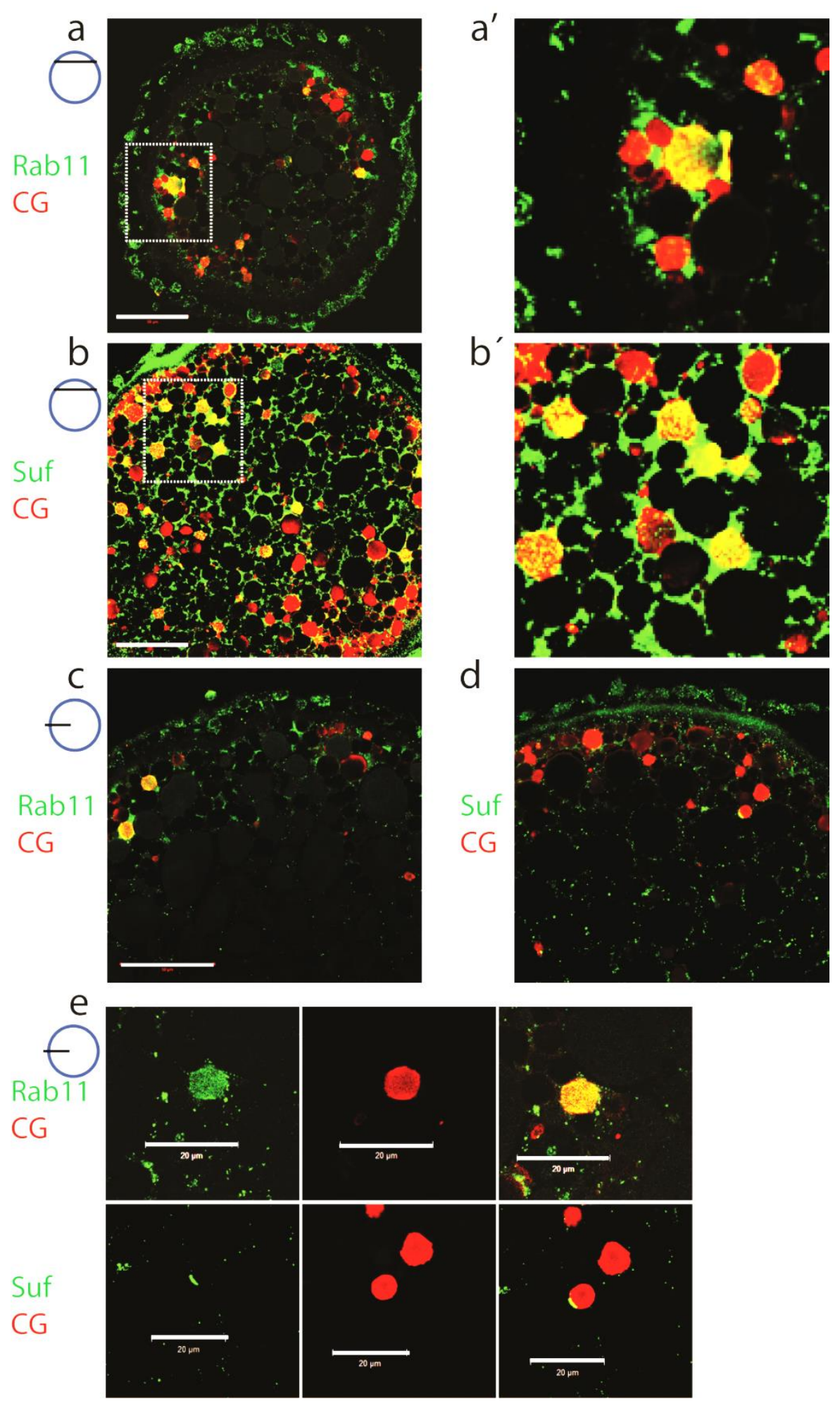


Figure 23. Rab11b and suf localizes to secretory granules in zebrafish oocyte.

a. Rab11b in green co-localize with secretory granule in red. Insert is magnified in a' and b. shows the co-localization between suf in green and secretory granule in red and the insert is magnified in b'. Panel $\mathrm{c}$ and d shows the differential staining pattern of rab11 on the surface of the vesicle which is magnified in panel e and suf staining small micro domains on secretory vesicle also magnified and in individual channel in panel e. Scale $-50 \mu \mathrm{m}$ (Panel a, b, c and d), scale $-20 \mu \mathrm{m}$ (panel e). Cartoon shows the optical section.

\subsubsection{Secretory granule accumulates in suf mutant oocytes}

Since the Rab11 positive vesicles accumulate in suf mutant, to analyze the role of Suf/Spastizin in cortical granule formation during oogenesis, we compared wildtype and mutant oocytes using electron microscopy. More cortical granules were visible in Suf/Spastizin oocytes (Fig. 9a). Interestingly, their electron dense core was not visible, which is a remarkably strong phenotype compared to other factors involved in dense-core vesicle maturation (Asensio et al.,2013). To verify an increase in cortical granules, we stained oocytes with MPA-lectin (Becker and Hart.1999). Wild type zebrafish oocytes showed a few, large cortical granules in the cytoplasm and numerous, smaller vesicles at the cortex. Conversely, the mutants also appeared to be filled with MPA-lectin indicating that Soufflé/Spastizin is involved in cortical granule formation during oogenesis (Fig. 9b). Although the number of granules are more but the quantification of cortical granule size from the electron microscopy image showed no difference between wild type and mutant (Fig. 9c). These results suggest that suf regulates the secretory granule formation.

\section{Figure 24. Suf mutant accumulates secretory granule.}

a. Electron micrograph showing cortex region of the oocyte with lysosome in dark indicated by arrow and grey secretory granule with white arrowhead showing the densecore region in the middle which is absent in the suf mutant oocyte. b. Confocal image of the oocyte before activation (Top panel) and after activation (Lower panel). Secretory granules are in red. Left panel is from wild type and right panel from mutant showing accumulation of secretory granule even after egg activation. Scale $-50 \mu \mathrm{m}$. c. Bar diagram shows the mean diameter of the secretory granule from wild type and mutant oocytes measured using electron microscopy picture. N-Number of oocyte sections counted, NS-Non significant. 

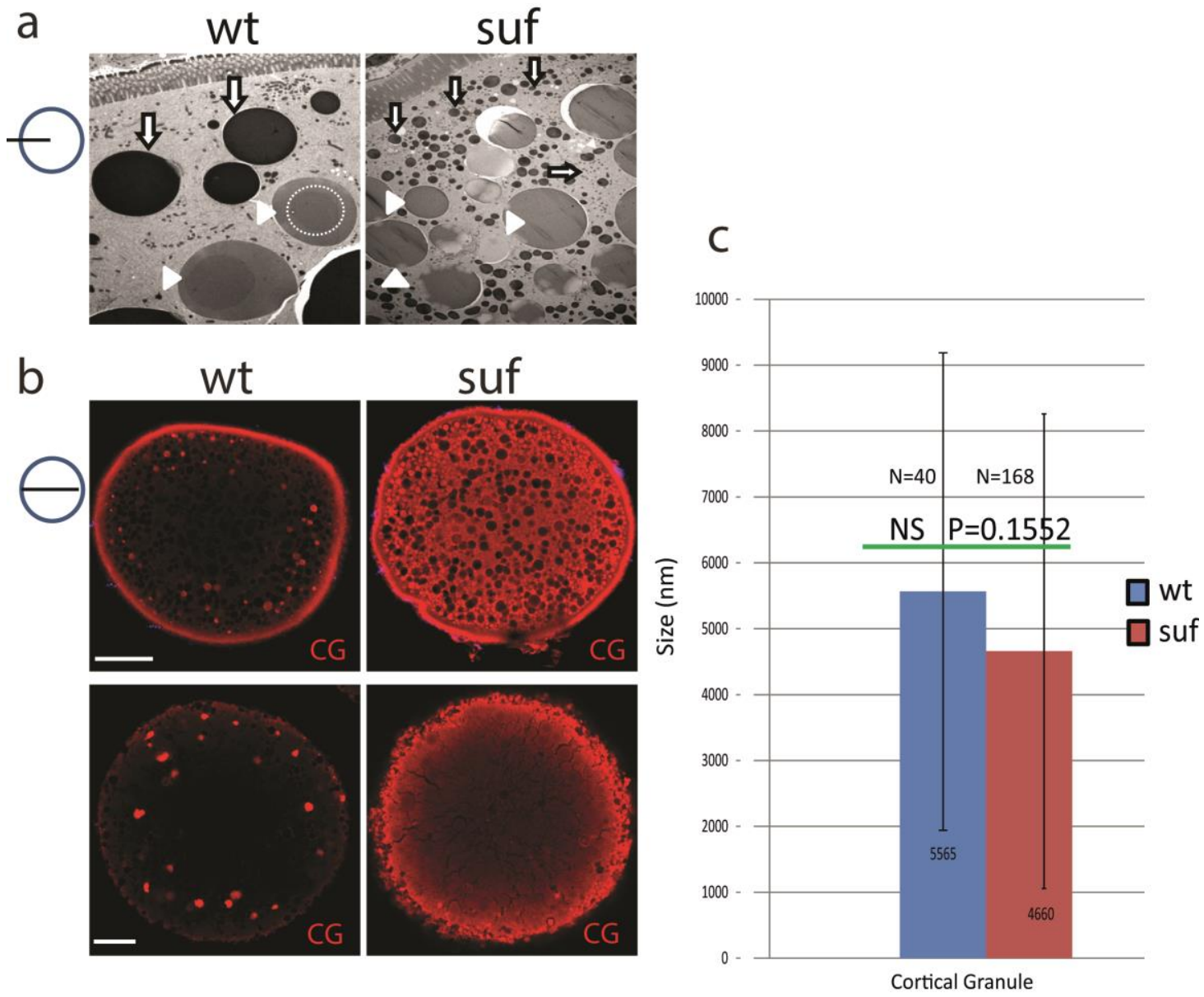

\subsection{Suf mutant failed to elevate the chorion}

\subsubsection{Role of cortical granule during fertilization}

The secretory granules are secreted from Golgi and they are transported to oocyte cortex hence called cortical granules. Immediately after fertilization, the granules fuse with oocyte membrane and deliver their cargo into the perivitelline space. The most prominent cargoes of cortical granules are carbohydrates, which after secretion increase their volume by hydration leading to chorion elevation and thereby create the perivitelline space between oocyte and chorion (Fig.10a). As in mammals, cortical granules are secreted after fertilization during a process termed "cortical reaction" and the induced chorion elevation is important to inhibit lethal polyspermy and mechanical damage to the embryo (reviewed in Wessel et al.,2001; Liu.2011).

\subsubsection{Suf mutant eggs fail to elevate the chorion after activation}

The observed additional granules in zebrafish mutants could be generated by two alternative mechanisms. Either Suf/Spastizin could regulate the formation of granules, 
which in the mutant leads to an increase of immature, non-fusogenic vesicles. Alternatively, Suf/Spastizin could regulate the sorting of the MPA epitope, which in the mutant oocyte leads to an increased number of MPA-positive vesicles. To check if the accumulated granules are functional and if they are functional, the mutant should have a bigger chorionic space than wild type. However, after triggering exocytosis in wild type and Suf/Spastizin stage $\mathrm{V}$ eggs by $\mathrm{H}_{2} \mathrm{O}$ exposure, we observed no chorion elevation $10 \mathrm{~min}$ after egg activation in mutants (Figure 10b). Furthermore, in embryos from mutant mothers $30 \mathrm{mpf}$ (minutes post fertilization) the chorion was weakly elevated (Fig. 10c), although the regular exocytosis process is completed within six minutes after sperm entry (Hart and Yu.1980). I quantified chorion and embryo diameters to exclude growth defects during oogenesis as cause for the size differences. Embryo size in wild type and mutants were similar, while chorion diameters were reduced in embryos from mutant mothers, which was also apparent in eggs activated without sperm, supporting the idea that chorion elevation is impaired (Fig. 10d, e, f and g). These results indicate that Suf/Spastizin controls the sorting of MPA-positive cargo, whose failure leads to the accumulation of immature vesicles in the mutant egg. This hypothesis predicts that immature cortical granules in the mutant egg do not secrete their cargo. Indeed, after activation, mutant eggs still contained numerous cortical granules; whereas wild type lost their vesicles close to the cortex with a few left in the inner cytoplasm (see Fig. 9b). These results together confirm that the suf mutant accumulate non- functional secretory granules and failed to complete exocytosis.

\section{Figure. 25 Suf mutant fail to elevate the chorion.}

a. Cartoon showing cortical reaction/Chorion elevation event. Secretory granule in red in the oocyte in left panel and right panel showing after activation where chorionic space is elevated. b \& c shows light microscopy image of activated egg and embryo respectively. Left panel from wt and right panel from suf mutant. d, e, f \& $g$ shows the diameter of the whole embryo, only chorionic space, only embryo without the chorion and only egg size. Small cartoon at the top shows how the measurement was made in different color. Panel $\mathrm{d}$ and e shows the difference in chorion elevation in the embryo and panel $g$ shows the chorion elevation difference in the egg. Scale $-50 \mu \mathrm{m}$. 
a
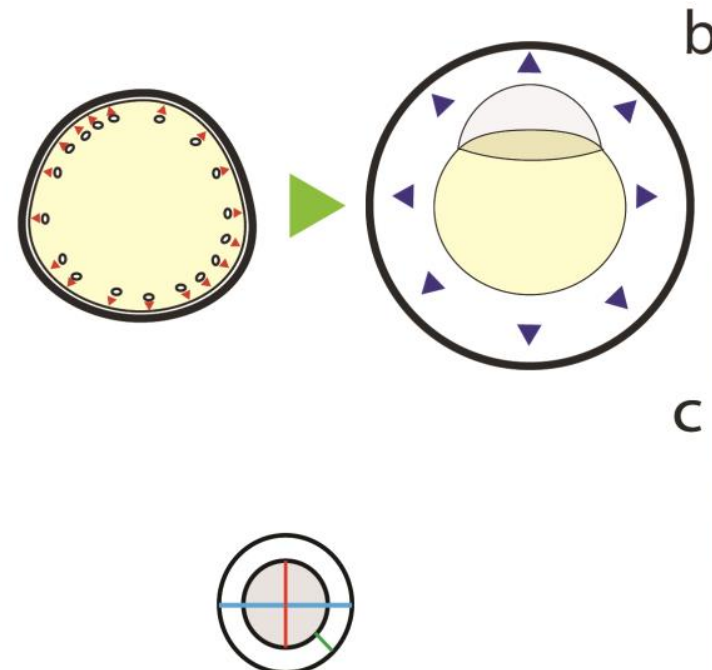

$\mathrm{d}+\mathrm{g}-$ Whole embryo/egg

e - Chorion elevation

$f$-Embryo without chorion

d

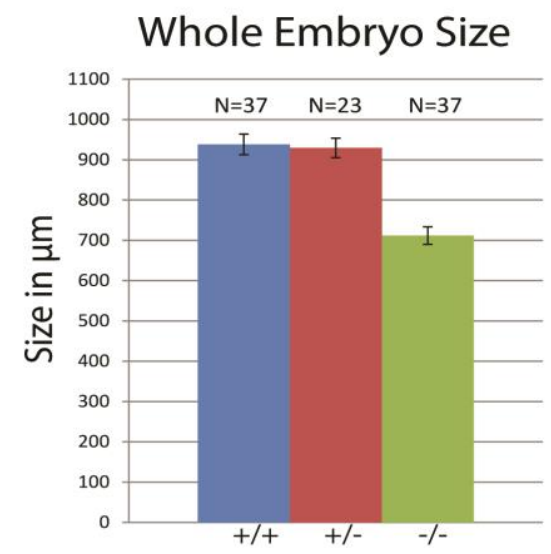

f

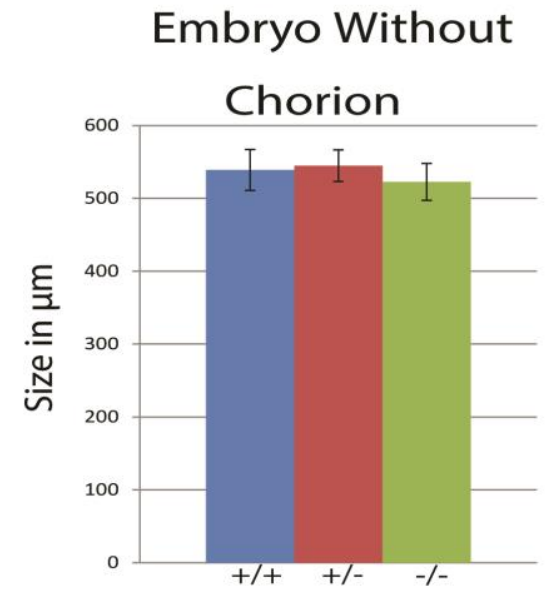

b
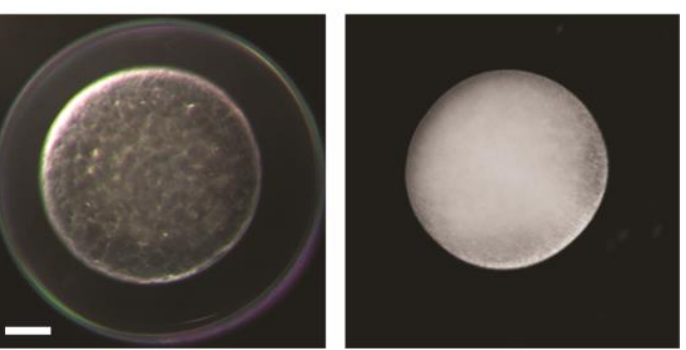

C
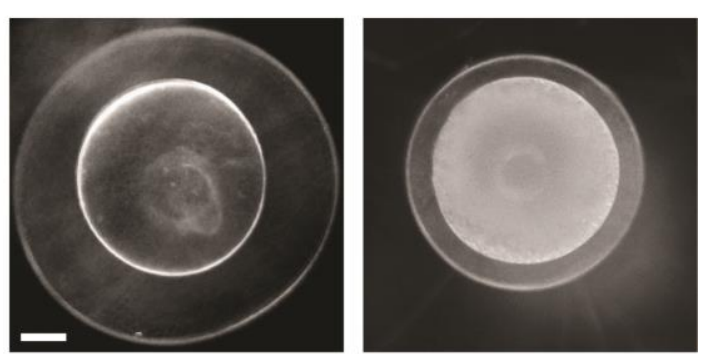

e

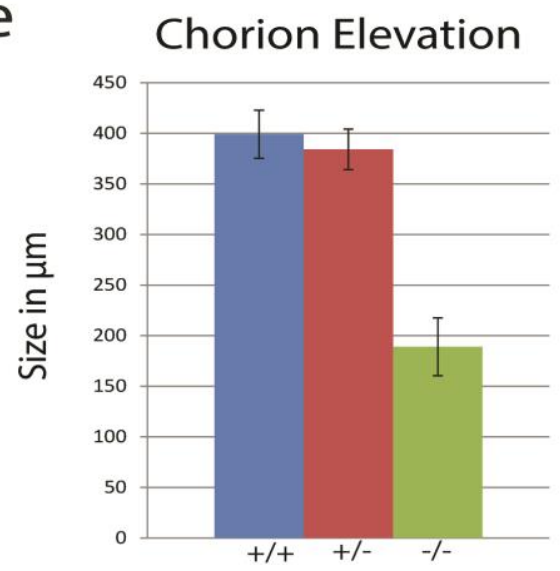

9

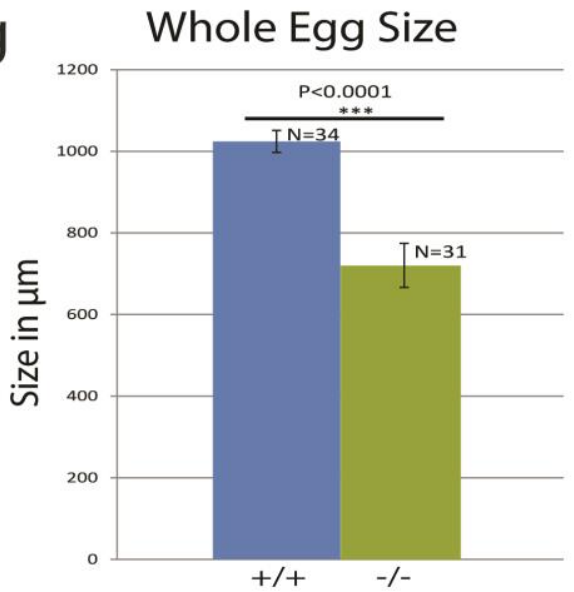

\subsubsection{Re-organization of $\mathrm{F}$-actin did not happen in suf mutant.}

To directly visualize the fusion of the vesicles, I analyzed the kinetics of exocytosis by labeling cortical f-actin (Becker and Hart.1999). The actin network reorganizes to allow exocytosis of vesicle and 60 seconds after activation fusing cortical granules generate 
negatively stained crypts in the wildtype actin meshwork, whereas suf mutants show no vesicle exocytosis (Fig. 11). 180 seconds after activation the collapsing crypts form scars of accumulating actin in wild-type oocytes, while the cortex of suf mutants still did not change. These experiments demonstrate that in the suf mutant cortical granules are not fusogenic and support the hypothesis that Suf controls their maturation.

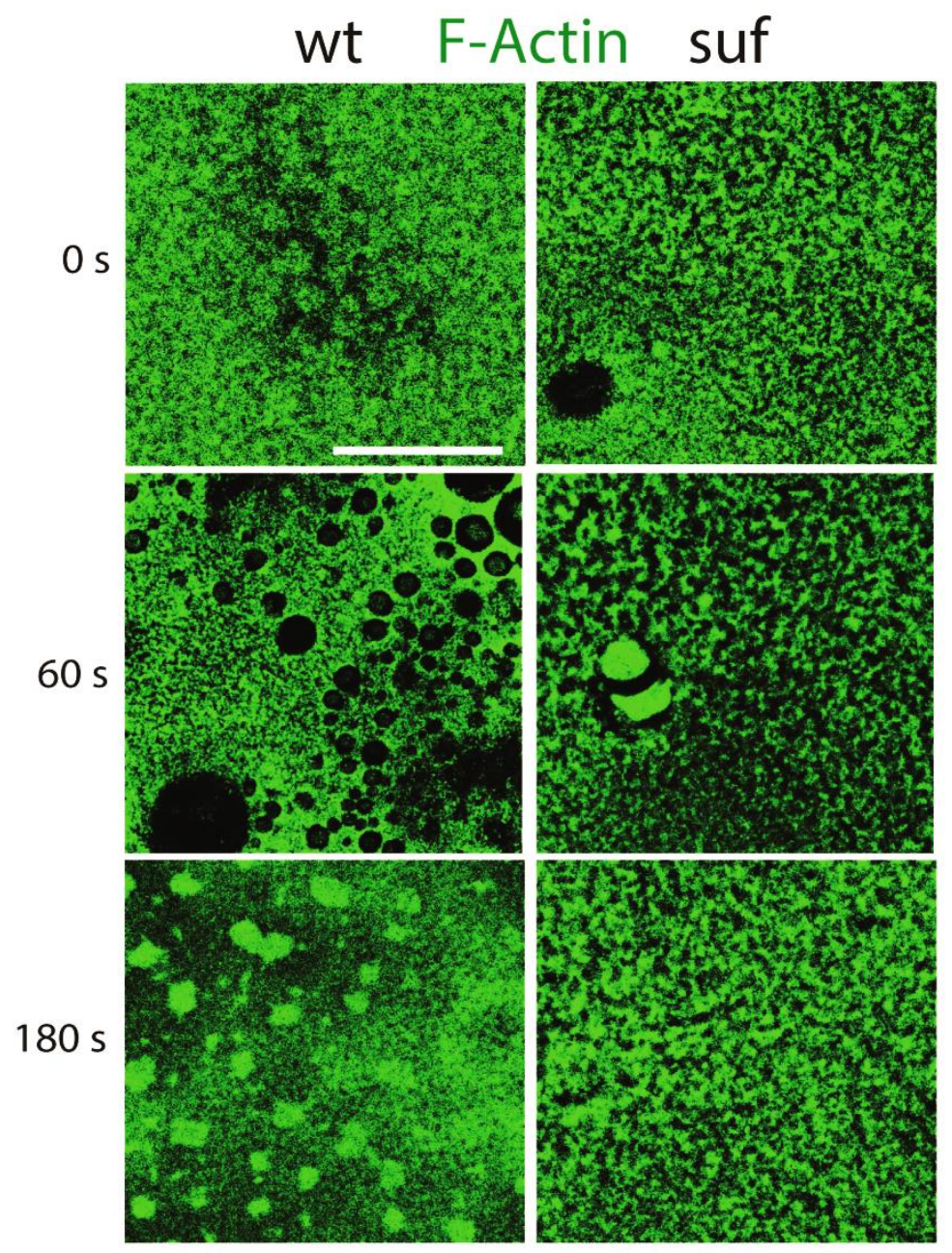

Figure 26. Suf mutant fail to reorganize the cortical F-actin.

Confocal image shows the F-actin distribution after egg activation from wild type and suf mutant eggs at different time points. Left panel is from wild type and right panel is from mutant. F-actin in green. Wild type shows the dynamic change in actin distribution during egg activation and exocytosis of the vesicle at 60s and presence of scar after exocytosis at 180s which did not happen in suf mutant eggs. Scale $-50 \mu \mathrm{m}$. 


\subsection{Suf regulates secretory granule maturation into dense-core vesicle (DCV)}

\subsubsection{Secretory granule maturation}

The immature secretory granule (ISG) goes through cargo sorting and removes some of the cargos, which are destined to other internal compartments like lysosome by vesicle fission that matures into fusion competent dense core vesicle (Fig.12a).

\subsubsection{Suf mutant accumulates immature secretory granule (ISG)}

In cell culture, immature secretory granules remove specific SNARE proteins from their cytoplasmic surface to acquire the competence to fuse with the plasma membrane (Steegmaier et al.,1999; Eaton et al.,2000; Ahn et al.,2010). For instance, depletion of GGA3 in neuroendocrine cells inhibits sorting of VAMP4 away from the neuroendocrine secretory granules, which needs to be removed to permit a vesicle to mature and fuse with the membrane (Kakhlon et al.,2006). To investigate if the suf mutant oocytes sorted out VAMP4 from ISG to mature into dense core vesicle, I analyzed VAMP4 in zebrafish oocytes. Suf mutants accumulated this VAPM4 SNARE protein compared to the wild type oocytes (Fig. 12b). These results show that in zebrafish oocytes Suf/Spastizin is essential for removing other cellular organelle cargoes for the maturation into fusion-competent cortical granules. This result also suggested that the suf mutant also fails to sort some of the cargo. To check if the sorting process finished successfully, I used electron microscopy. Surprisingly, the secretory granules missed inner dense core in the suf mutant (Fig.12c), confirming that suf mutants failed to complete the sorting process successfully. Taken together, all these results confirmed that suf regulates cortical granule maturation by successful sorting of the cargoes to form the dense core vesicle. 
a
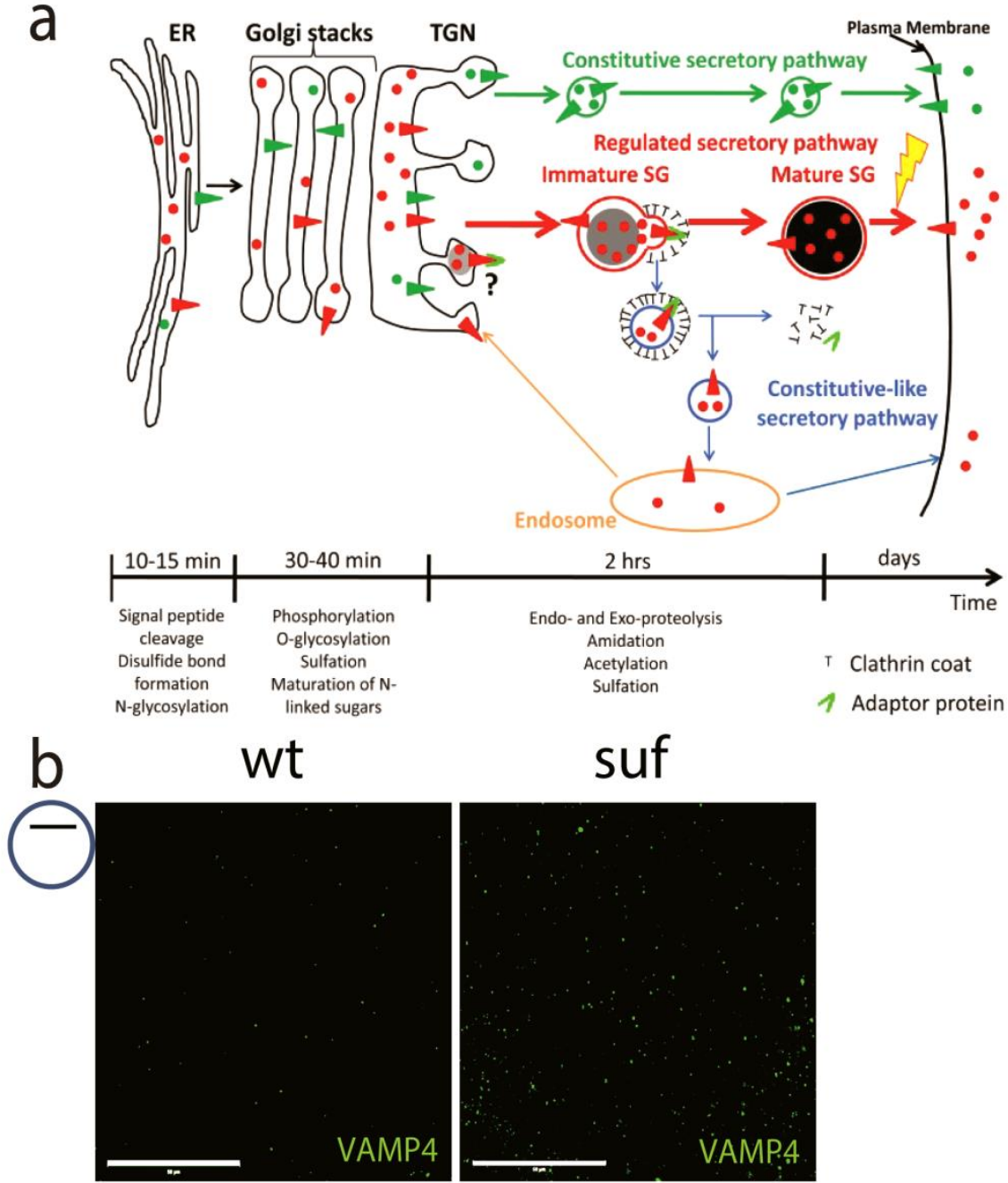

suf

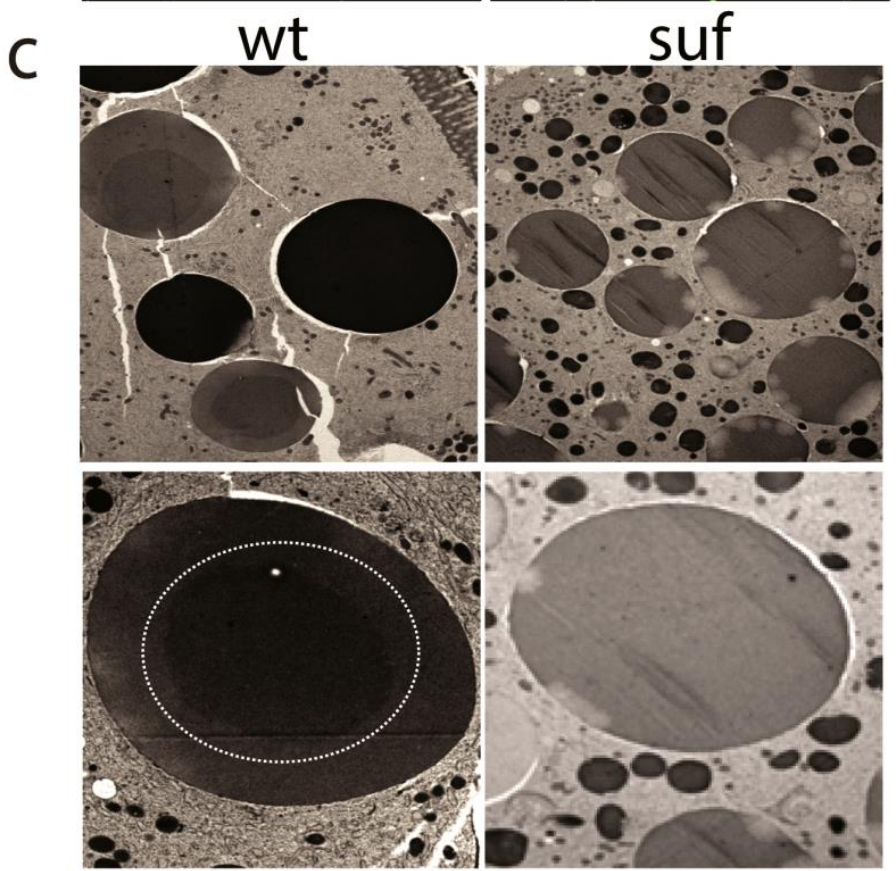


Figure 27. Suf mutant oocyte accumulates immature secretory granule.

a. Cartoon shows the three possible ways of the pathway from Golgi. Green marks the constitutive secretory pathway and their cargos from Golgi, red indicates regulated secretory pathway and their cargos from Golgi while blue indicates constitutive like pathway which are vesicle budded from immature secretory granule during maturation into dense-core vesicle which indicates the successful maturation and sorting of their cargos. These vesicles are delivered to either endosomes or secreted out like constitutive pathway by which it connect the secretory pathway to endo-lysosomal system. b. Antibody staining for immature secretory granule marker VAMP4 in green. Left panel from wild type and right panel from suf mutant. Scale $-50 \mu \mathrm{m}$. c. Electron micrograph from wt and mutant oocytes showing secretory granule with dense core in the wild type and without in suf mutants. Dashed circle in the bottom panel marks the dense-core region in wt. Left side image from wt and right side image from suf mutant.

\subsection{Suf is necessary for vesicle fission from immature secretory granule (ISG) during secretory granule maturation}

\subsubsection{Suf mutant accumulates cortical compartment with clathrin buds}

In cell culture experiments, VAMP4 is removed from immature secretory granules after it is sorted into Clathrin-coated buds, which finally pinch off (Orci et al.,1985; Tooze and Tooze.1986; Steegmaier et al.,1999). The localization of Suf to compartmental microdomains also supports a role for Suf in sorting. Consistent with my previous results, tissue culture experiments proposed a role for Suf in sorting (Hirst et al.,2013a), which is also a prerequisite for vesicle abscission. To analyze in zebrafish oocytes whether Suf/Spastizin mutants show a defects in vesicle abscission, I returned to the EM analysis and investigated mutants at higher magnification. Interestingly, close to the cortex of mutant oocytes we discovered compartments, which were covered with Clathrin-coated buds, which appeared to not complete budding and abscission (Fig. 13 (white arrowheads)). In wild type oocytes these prominent compartments with Clathrin-buds were never observed suggesting that Suf/Spastizin controls a step such as sorting to initiate vesicle budding and fission. 
Figure 28. Suf mutant oocyte accumulates Clathrin buds on the vesicular compartment.

Electron micrograph showing the ultra-structures of oocyte cortex region from wild type (Left panel) and suf mutant (Right panel). Notice the big vesicle with lot of clathrin buds on the membrane, which are not cleaved yet in the mutant oocytes. Arrowhead indicates the clathrin bud. Scale - top panel - $0.5 \mu \mathrm{m}$, left middle and bottom $-1 \mu \mathrm{m}$ and right middle and bottom $-2 \mu \mathrm{m}$.
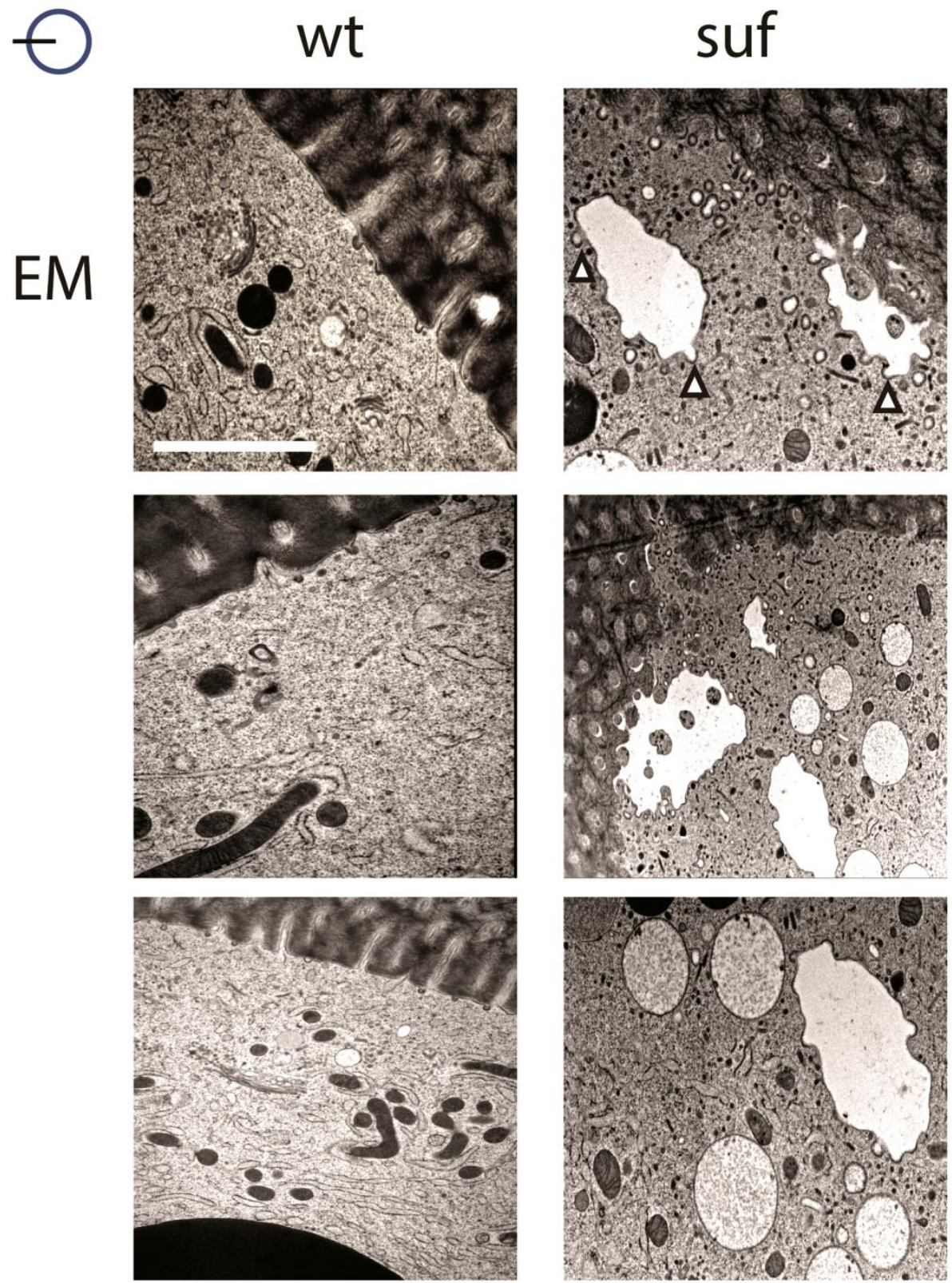

\subsubsection{Dynamin accumulates on secretory granule in suf mutant}

To confirm that Clathrin-coated buds accumulate, we compared the localization of Clathrin in wild type and mutant oocytes. Clathrin accumulated in mutants corroborating 
the EM data (Fig. 14a). The compartments accumulating in mutant oocytes looked similar to cisternae formed in the temperature-sensitive Dynamin mutant shibire in Drosophila (Pelissier et al.,2003). Dynamin exerts the ultimate step after sorting and budding during the fission process (Praefcke and McMahon.2004; Schmid and Frolov.2011; Ferguson and De Camilli.2012). To analyze if Dynamin also accumulates in the suf mutant, I checked Dyanmin in suf mutant oocytes and indeed, I observed an accumulation of Dynamin on cortical granules suggesting that Suf/Spastizin controls a molecular step, which permits Dynamin mediated fission in oocytes (Fig.14b). These results together confirm that suf regulates cargo sorting coupled with fission, which leads to cortical granule maturation.

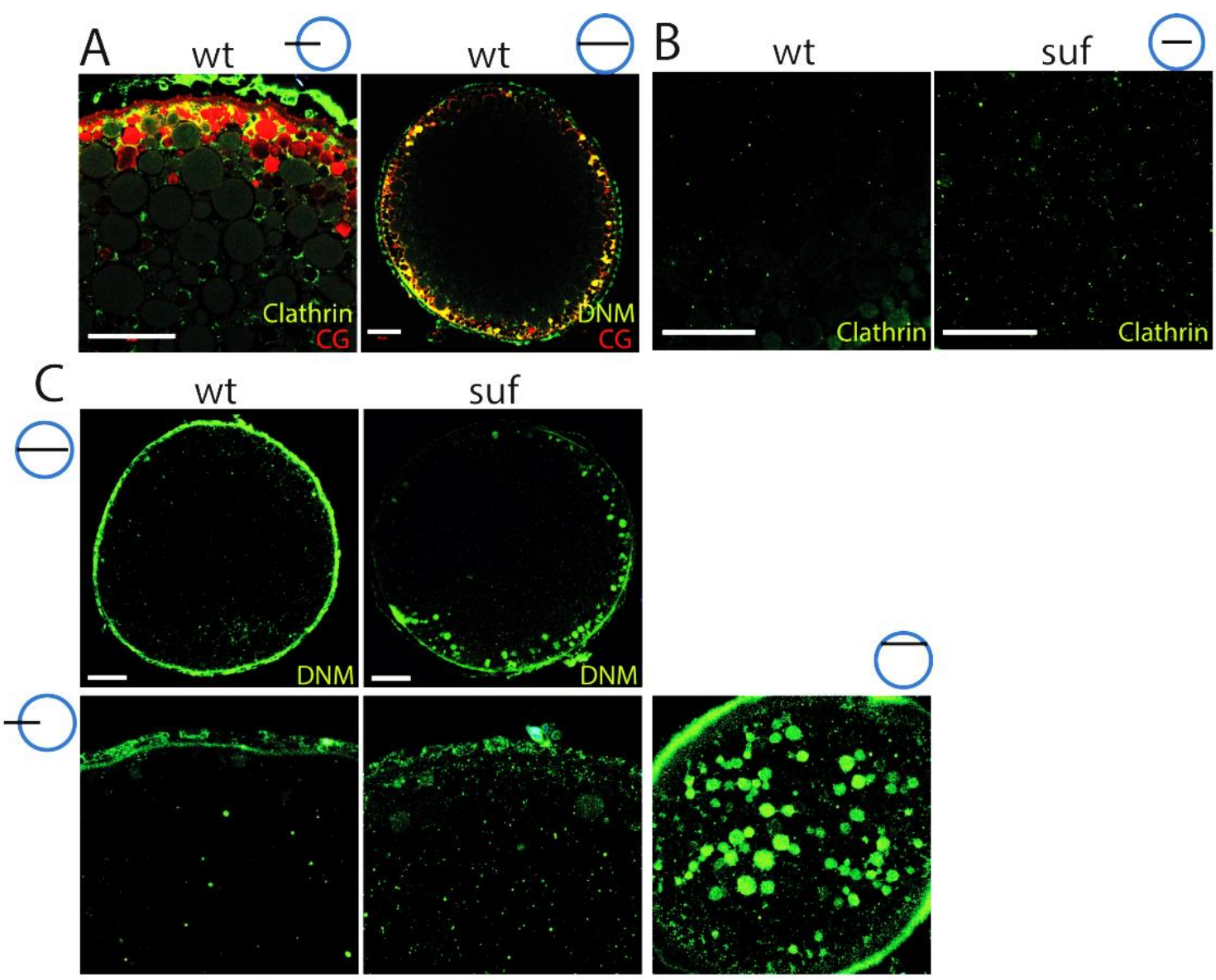

Figure 29. Suf mutant oocytes accumulate clathrin and Dynamin.

a. Double staining for secretory granule in red with Clathrin and Dynamin in green in wildtype oocytes. Confocal image shows positive for both proteins on secretory granule. $b$. Staining for clathrin in green from wild type and suf mutant oocytes showing accumulation of clathrin in the mutant oocytes. c. Staining for Dynamin in green in wild type and suf mutant oocytes showing accumulation of dynamin on secretory granule in the suf mutant. Small cartoon on the top of the image shows the optical section. Scale $-50 \mu \mathrm{m}$. 


\subsection{Chemical inhibition of Dynamin function mimics suf mutant phenotype}

If fission by Dynamin is essential for cortical granule maturation, treatment of zebrafish oocytes with the Dynamin-specific inhibitor Dynasore should mimic the mutant phenotype, if Suf is required genetically upstream of vesicle fission. Fascinatingly, wt oocytes accumulated the cortical granule marker MPA similar to mutants after Dynasore treatment (Fig. 30a). However, we also noted differences such as mature cortical granules in Dynasore-treated wt oocytes, which probably formed during oogenesis before the drug treatment (Fig. 30b). Although Dynamin is involved in additional processes as revealed by the Dynasore inhibition, these data confirm that Dynamin mediated fission is required during maturation of secretory granules in the zebrafish egg.

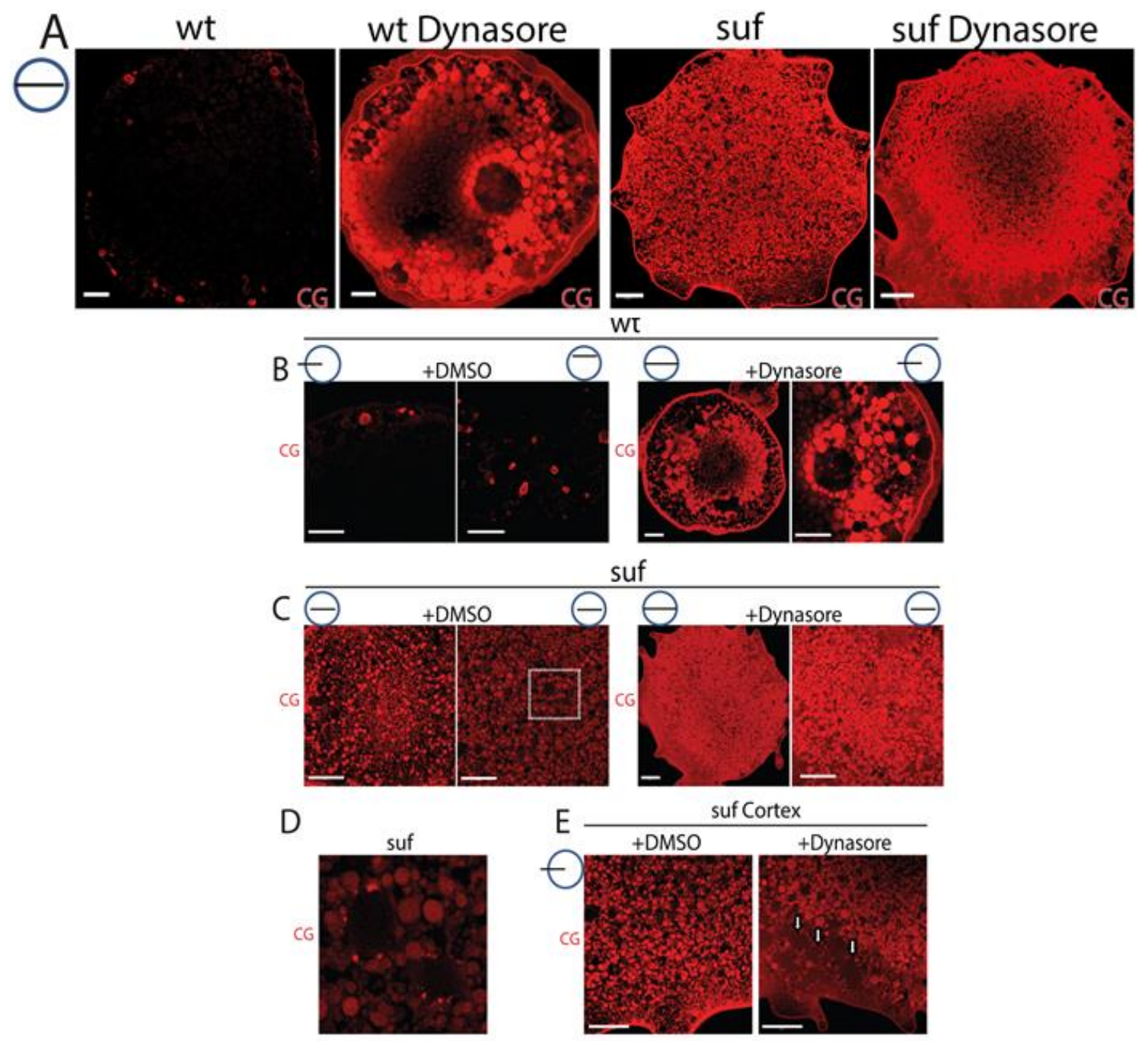


Figure 30. Dynasore treated oocyte mimics soufflé phenotype.

a. Staining for secretory granule (Red) in the oocyte from wt, suf mutant and dynasore treated from wt and suf mutant. b \& c shows the change in the structure of the secretory granule in DMSO and dynasore treated oocytes from wildtype and suf mutant at different optical plane. The insert in panel $\mathrm{c}$ is magnified in panel $\mathrm{d}$ showing the cargo accumulating at the margin of the vesicle instead of accumulating in the middle. e. Shows the difference in the cortex region of suf oocytes treated with DMSO and dynasore. Arrow indicates many vesicle like shown in panel d accumulating in dynasore treated suf mutant oocytes. Scale $50 \mu \mathrm{m}$.

\subsubsection{Inhibition of Dynamin obstructs chorion elevation in wildtype eggs}

Cortical granule formation was previously considered to be an ongoing process during oogenesis, but whether it continued after ovulation remained unclear (Wessel et al.,2001). To analyze whether cortical granule maturation still continues in ovulated, fully matured stage $\mathrm{V}$ eggs, and also to check in-vivo if dynamin inhibition can inhibit chorion elevation, we treated the wild-type ovulated eggs for 5, 15 and 30 min with Dynasore and examined chorion elevation. Indeed, the level of chorion elevation corresponded well with the duration of Dynasore treatment (Fig. 31a \& b). Remarkably, the 30 min treatment with Dynasore inhibited chorion elevation completely. Most importantly, Dynasore also reverted the transparency of the egg cytoplasm back to opaqueness similar to suf mutant egg. The transparency of the egg cytoplasm is caused by changes in yolk globules, which are considered dormant lysosomes (Yamamoto and Oota.1967; Wallace and Selman.1990; Le Menn et al.,2007). These intriguing results suggest that cortical granule maturation is critical for the function of lysosomal yolk globules during all stages of oogenesis. In summary, our results show that Suf/Spastizin is a key regulator of secretory vesicle maturation during zebrafish oogenesis, probably before the fission of Clathrin-coated buds through Dynamin.

\subsubsection{Dynasore treated eggs phenocopy soufflé mutant at molecular level}

To investigate if dynasore treated wild-type egg also show soufflé like phenotype at molecular level, I analyzed the dynasore treated eggs for cortical granule and immature secretory granule marker VAMP4 since both accumulates in the suf mutant. Indeed the dynasore treated wild-type eggs accumulates both MPA-lectin and VAMP4 (Fig.31c) confirming that inhibiting dynamin mimics soufflé phenotype at morphological and molecular level. 
A

Co
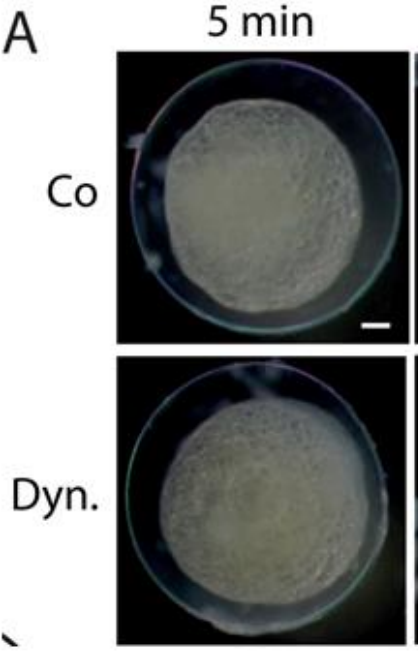

$15 \mathrm{~min}$
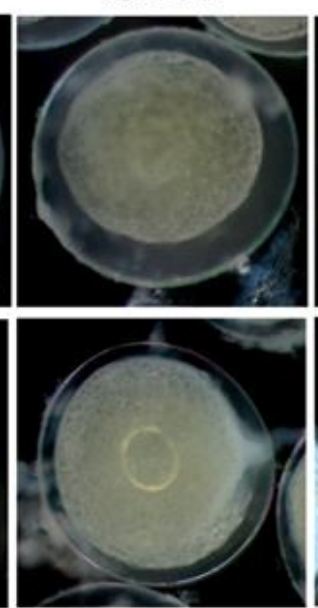

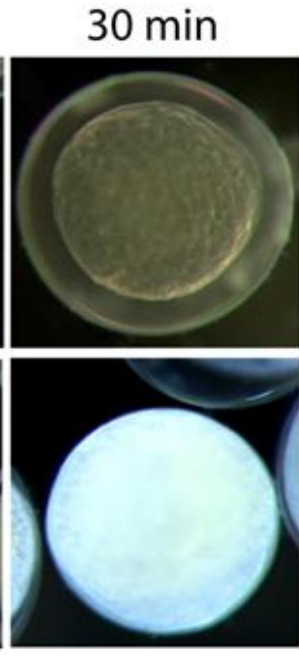

B

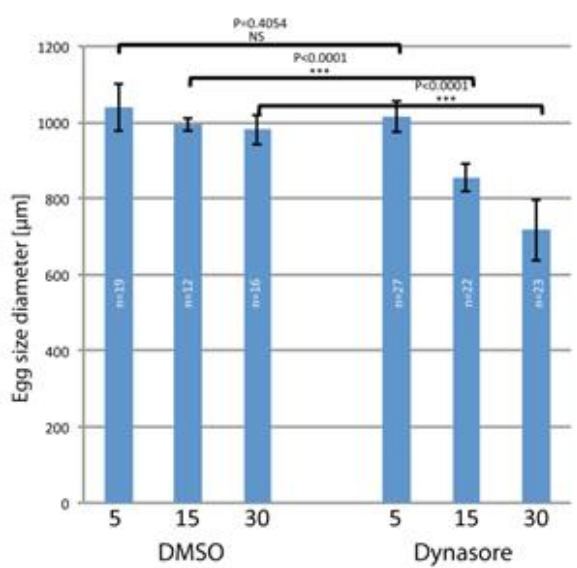

C

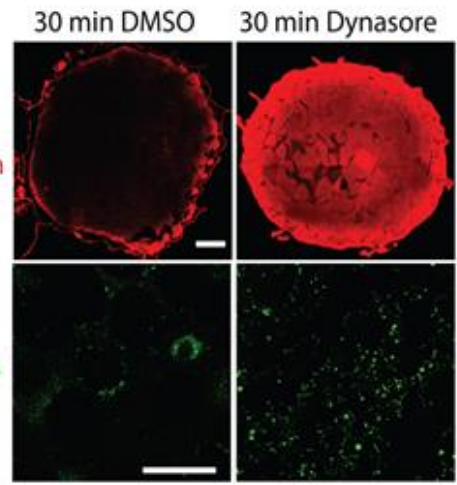

Figure 31. Dynasore treated egg mimics suf mutants functionally.

a. Light microscopy image showing chorion elevation of wild-type eggs treated with dynasore or DMSO treated for specific time as indicated in the image. Top panel from wild type from DMSO treated and bottom panel from wild type treated with dynasore. $b$. Bar diagram shows the mean size of the embryo after chorion elevation at different time points from DMSO and dynasore treated eggs. c. Staining for VAMP4 in green and secretory granule in red shows the accumulation both in dynasore treated wt eggs similar to suf mutant oocytes and eggs. Scale $-50 \mu \mathrm{m}$.

\subsection{Suf is required for mitosis but not for meiosis}

\subsubsection{Suf/Spastizin is not required during meiosis}

Since recycling was not disrupted in suf oocytes, we examined additional processes requiring recycling endosomes during oogenesis. Endosomal recycling is involved in meiotic maturation in C. elegans (Cheng et al.,2008) and Xenopus laevis (El-Jouni et al.,2007), which is induced in teleost oocytes by $17 \alpha, 20 \beta$-dihydroxy-4-pregnen-3one(DHP) (Jalabert et al.,1978; Nagahama.1985; Selman et al.,1994). Staining of the germinal vesicle with fluorescent Phalloidin by Dr. Amandine-Stein revealed that 
Suf/Spastizin oocytes underwent GVBD (germinal vesicle breakdown) in response to DHP $(93 \%, n=28)$ similar to wild-type oocytes $(82 \%, n=17)$, whereas ethanol-treated controloocytes retained their germinal vesicle (suf: 64\%, n=11; wt: 57\%, n=28) (Fig. 32a, b). This finding was also confirmed by following the dynamics of GVBD in vivo with a transgenic H2A-GFP (Histone2A-GFP) reporter line (Pauls et al.,2001) (Fig. 17c). We also examined polar body extrusion (Fig. 32d) and spindle formation (Fig. 32e) during oocyte maturation, but observed no difference between wild type and mutant. These results show that Suf is not required for meiotic maturation in the zebrafish oocyte.

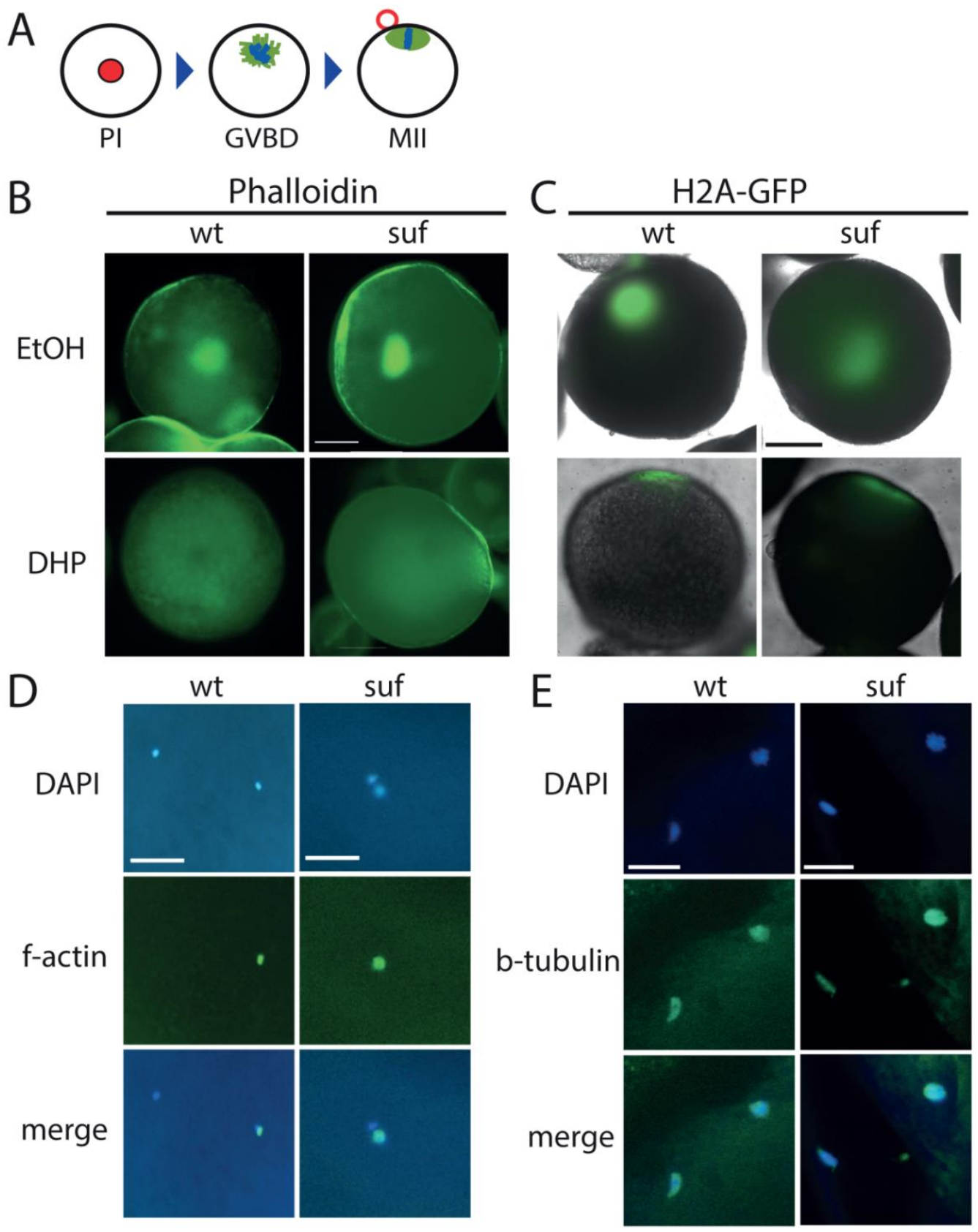




\section{Figure 32. Suf is not required for meiosis.}

a. Cartoon shows the meiotic maturation process during oogenesis from prophase I to metaphase II. Zebrafish oocytes are arrested in prophase I (PI) of the first meiotic cell cycle indicated by the huge germinal vesicle (red). Maturation initiates with germinal vesicle breakdown (GVBD) and the first meiosis leads to the formation of a polar body (red circle). The egg arrests again in metaphase of the second meiotic cell cycle (MII), ready to be fertilized. b \& c shows the germinal vesicle break down in wild type and suf mutant oocytes. b. Phalloidin staining in green from the oocytes treated with either ethanol or DHP. Bottom panel shows the disappearance of germinal vesicle after DHP treatment

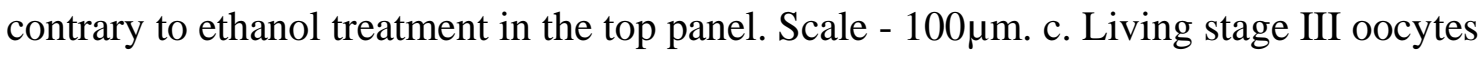
from wt and Suf/Spastizin mothers treated with carrier or DHP. The chromatin is highlighted by a Histon2A-GFP transgene (green) showing that GVBD occurs at the same time in mutant oocytes as in wt, whereas the cytoplasm stays opaque in Suf/Spastizin oocytes. Scale $-100 \mu \mathrm{m}$. d. Panel d shows the polar body extrusion from wt and suf mutant oocytes. Actin in green to mark the polar body, nuclei in blue (DAPI) and merge at the bootom. e. Panel e shows the spindle formation in the oocyte from wt and suf mutant. Nuclei in blue and b-tubulin in green, which marks the spindle and merge at the bottom. Scale $-25 \mu \mathrm{m}$

\subsubsection{Soufflé embryos show defect in cytokinesis}

In eukaryotes, Golgi derived vesicles and Rab11 are involved in cytokinesis during mitosis (reviewed in Barr and Gruneberg.2007; Simon and Prekeris.2008; Schiel et al.,2013; Hehnly and Doxsey.2014). Consistently, after RNAi depletion of the Suf-homolog FYVE-Cent in HeLa cells, a cytokinesis defect was observed (Sagona et al.,2010). To analyze cytokinesis in embryonic cells with defective maternal Suf protein, I analyzed embryos from suf mutant mothers and the embryos showed defect in cleavage (Fig.33a). Amandine also labeled the cell cortex of 32-cell embryos with Phalloidin and their nuclei with DAPI (Fig.33b). Whereas embryos from wild-type mothers showed one nucleus per cell, age-matched embryos from suf mutants exhibited multinucleated cells. This result suggests that the maternally controlled cell cycles require Suf/Spastizin similar to the cytokinesis defect discovered in HeLa cells. There by, these results support the role of Suf in secretion in zebrafish oocytes. 
A

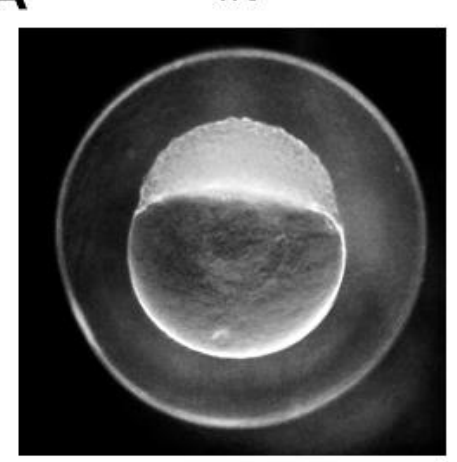

suf
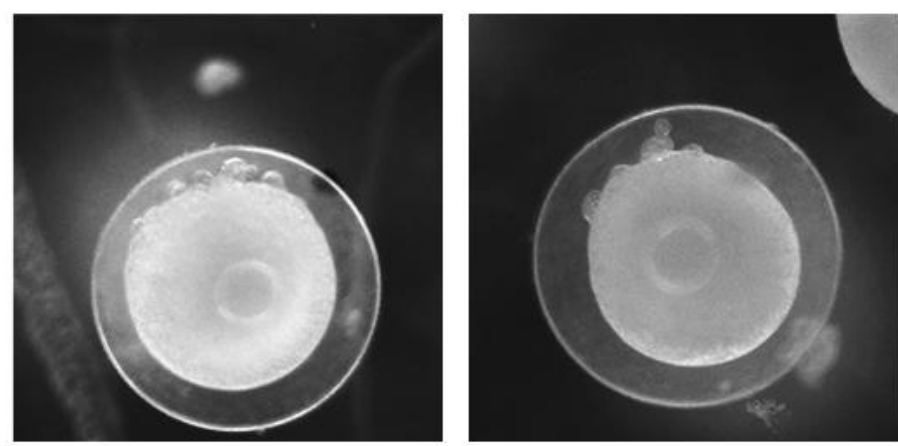

B

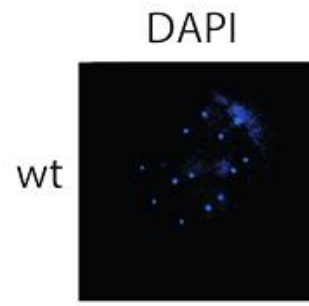

f-actin

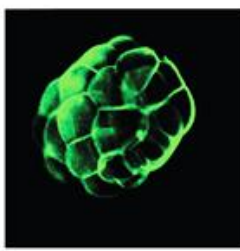

merge
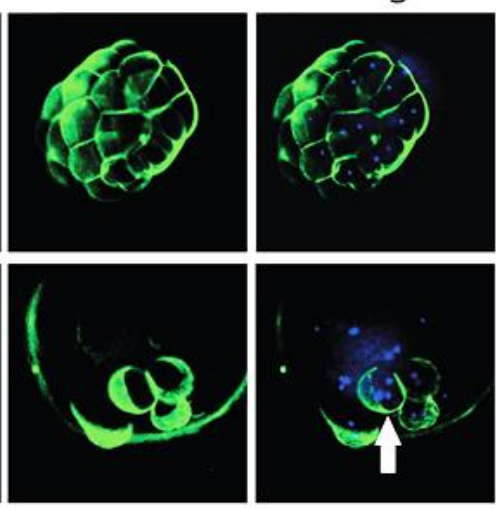

Figure 33. Suf mutant embryos shows a defect in cytokinesis.

a. Light microscopy image showing the defective cleavage in suf mutant embryo compared to the wild-type embryo in the left. b. Suf/Spastizin is required for cytokinesis. Embryos at 32-cell stage from heterozygous wt (top panel) or homozygous (bottom panel) Suf/Spastizin mothers with labeled nuclei (DAPI; blue) and plasma membranes (Phalloidin; green). In contrast to wt, few eggs from mutant mothers initiate cell division after fertilization, but then show cells with multiple nuclei (arrowhead).

\subsection{Injecting suf DNA in the mutant oocyte rescues the mutant phenotype}

\subsubsection{Suf DNA injection rescues chorion elevation phenotype}

Since chorion elevation provided a sensitive read-out for Suf functionality in zebrafish oocytes, I analyzed whether injecting wild-type Suf gene rescues the mutant phenotype. However, the length of the suf gene with almost $8 \mathrm{~kb}$ made it difficult to obtain sufficient in-vitro transcribed RNA for injection and hence, I injected plasmid DNA into oocytes (Clelland et al.,2007; Bontems et al.,2009; Nair et al.,2013). Furthermore, we used stage III oocytes, since they can be incubated for longer periods to allow for protein 
expression than matured stage $\mathrm{V}$ eggs. After $12 \mathrm{hrs}$, the majority of wt oocytes elevated their chorion $(84.3 \% \pm 6.9)$, whereas suf mutant oocytes rarely showed a perivitelline space (7.3\% \pm 2.9$)$ also after injection of control plasmid. Wild type Suf plasmid partially rescued chorion expansion in mutant oocytes $(58.5 \% \pm 19.0)$, but the chorion was not elevated to the same extend as seen in the wild type. Interestingly, when I overexpressed the p96re allele, I also observed partial rescue, but at a lower percentage (40.0\% \pm 13.4$)$ (Fig.34 a\&b). This result confirms that the suf gene could rescue the mutant phenotype partially and the suf mutant allele behaves as a hypo morph allele consistent with reduced chorionic space in the embryo after $30 \mathrm{mpf}$.
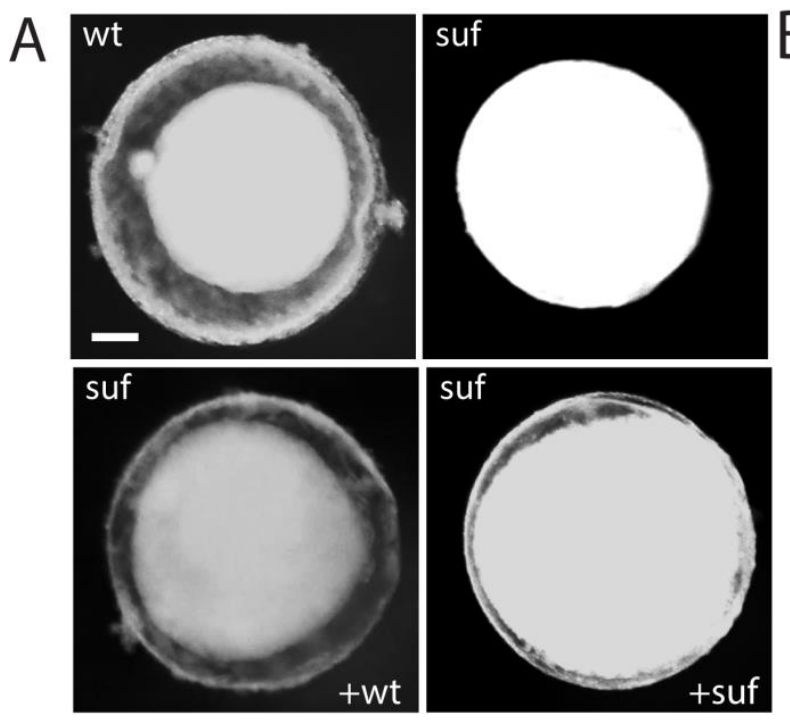

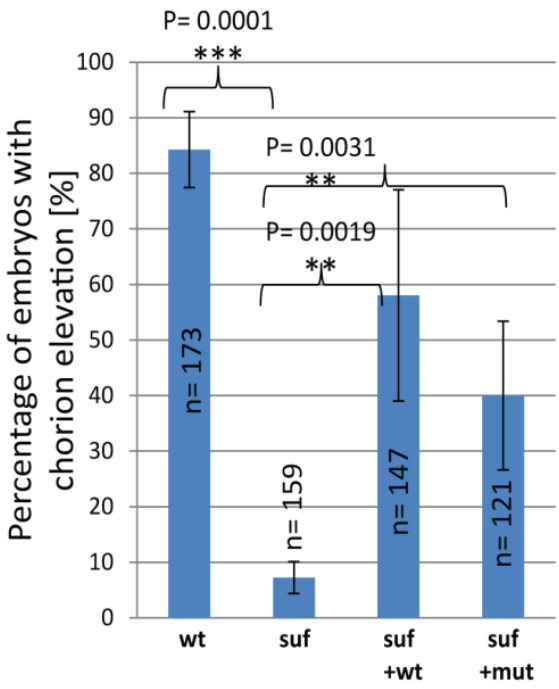

Figure 34. Suf DNA injection rescues suf mutant.

a. Stage III oocytes from $+/-(w t)$ or $-/-$ Suf/Spastizin mutants (mut) after $12-16 \mathrm{~h}$ incubation in L-15 medium in the top panel. Bottom panel shows stage III oocytes from -/- Suf/Spastizin mutants after injection of plasmid encoding wt or mutant Suf (p96re allele). Note that the mutant $S u f^{996 r e}$ injected oocytes also show chorion elevation similar to wt Suf injected oocytes to some extent. Scale $-50 \mu \mathrm{m}$. b. Bar diagram showing the percentage of the embryos with elevated chorion. Mutant oocytes injected with wt and the suf mutant construct rescue the chorion elevation.

\subsubsection{Suf gene injection also rescues the molecular phenotype}

To confirm the rescue with molecular markers, I stained the injected oocytes with MPA-lectin and VAMP4. Both markers were reduced in mutant oocytes after injection of plasmid encoding wild-type Suf or Suf ${ }^{\text {96re }}$, but the control plasmid did not change anything (Fig. $35 \mathrm{a} \& \mathrm{~b}$ ). This result upholds the phenotypic rescue and confirms our previous 
hypothesis that the zebrafish p96re allele encodes a hypo morph with reduced activity. Taken together these data demonstrate that Suf controls secretory vesicle maturation probably by sorting during zebrafish oogenesis.

\subsubsection{Soufflé regulates cortical granule/ secretory granule maturation}

Considering the above results, which leads to the conclusion that soufflé regulates cargo sorting from immature secretory granule and only successful sorting results in fusion competent dense core vesicle. These matured dense core vesicles are essential after fertilization for chorion elevation and modifying the membrane to avoid deadly polyspermy effect. The proposed model (Fig.36) shows the role of soufflé during secretory granule maturation and chorion elevation.

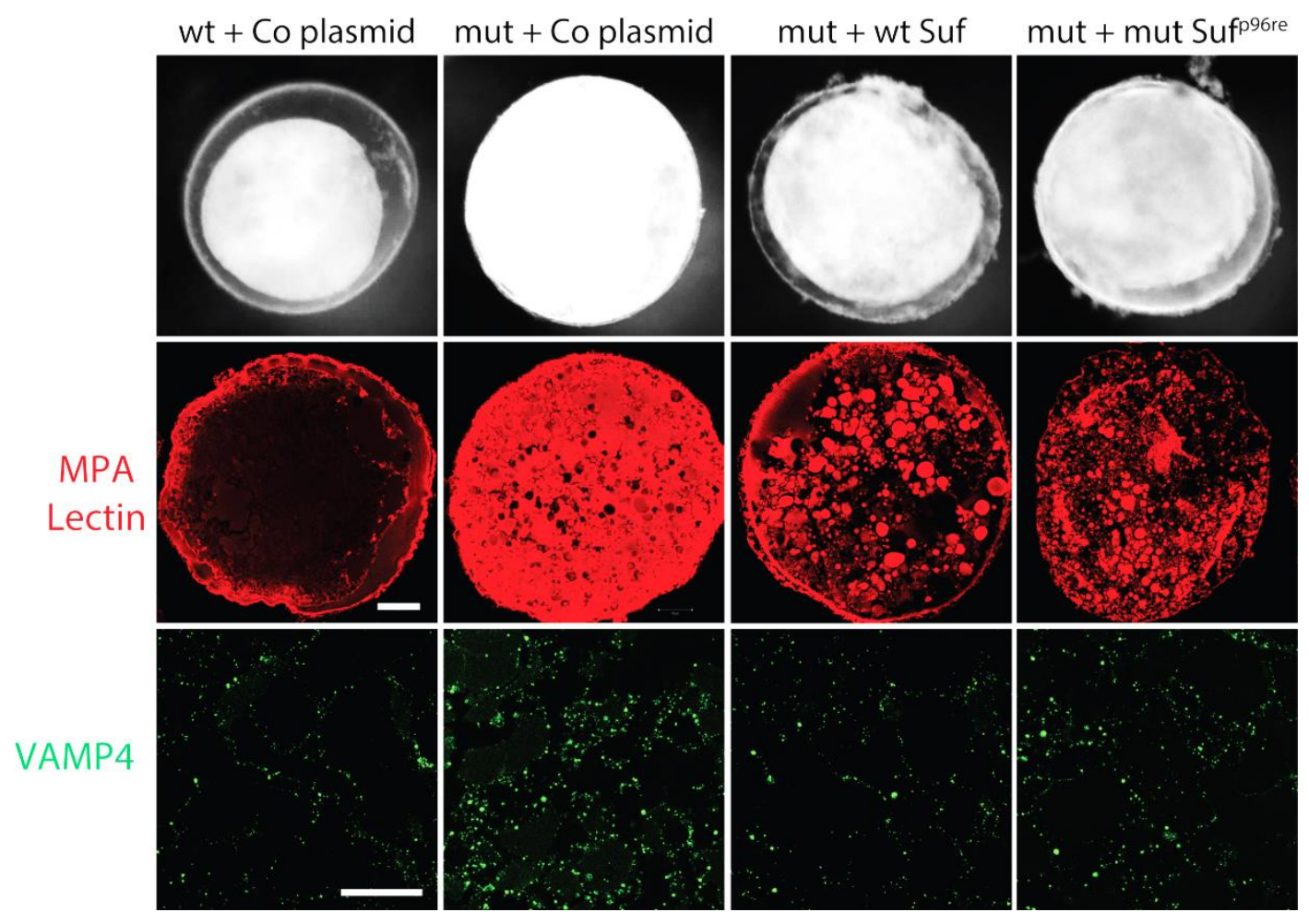

Figure 35. Suf DNA injection rescues the mutant phenotype at molecular level.

Morphological phenotype (upper row) of chorion elevation in activated wt ( $\mathrm{wt}+\mathrm{co}$ plasmid) $(100 \% ; n=55)$, but not in mut oocytes after injection with control DNA $(s u f+$ Co plasmid) $(0 \% ; \mathrm{n}=56)$. Mutant oocytes injected with plasmid encoding wt Suf (mut + wt Suf) $(87.5 \% ; \mathrm{n}=56)$ or mut Suf $f^{\mathrm{p} 9 \mathrm{re}}$ (mut + mut Suf $\left.{ }^{96 \mathrm{re}}\right)(67.9 \% ; \mathrm{n}=53)$ show chorion elevation. Rescue of MPA-lectin (middle row) and VAMP4 (lower row) accumulation on immature secretory granules after injection of plasmid encoding wt $S u f$ (mut + wt $S u f$ ) or mut $S u f^{p 96 \mathrm{re}}$ (mut + mut $S u f^{996 \mathrm{re}}$ ) into mutant oocytes. Notice the reduction the accumulation 
of VAMP4 and secretory granule after the injection of wt or suf mutant construct. Scale $50 \mu \mathrm{m}$.

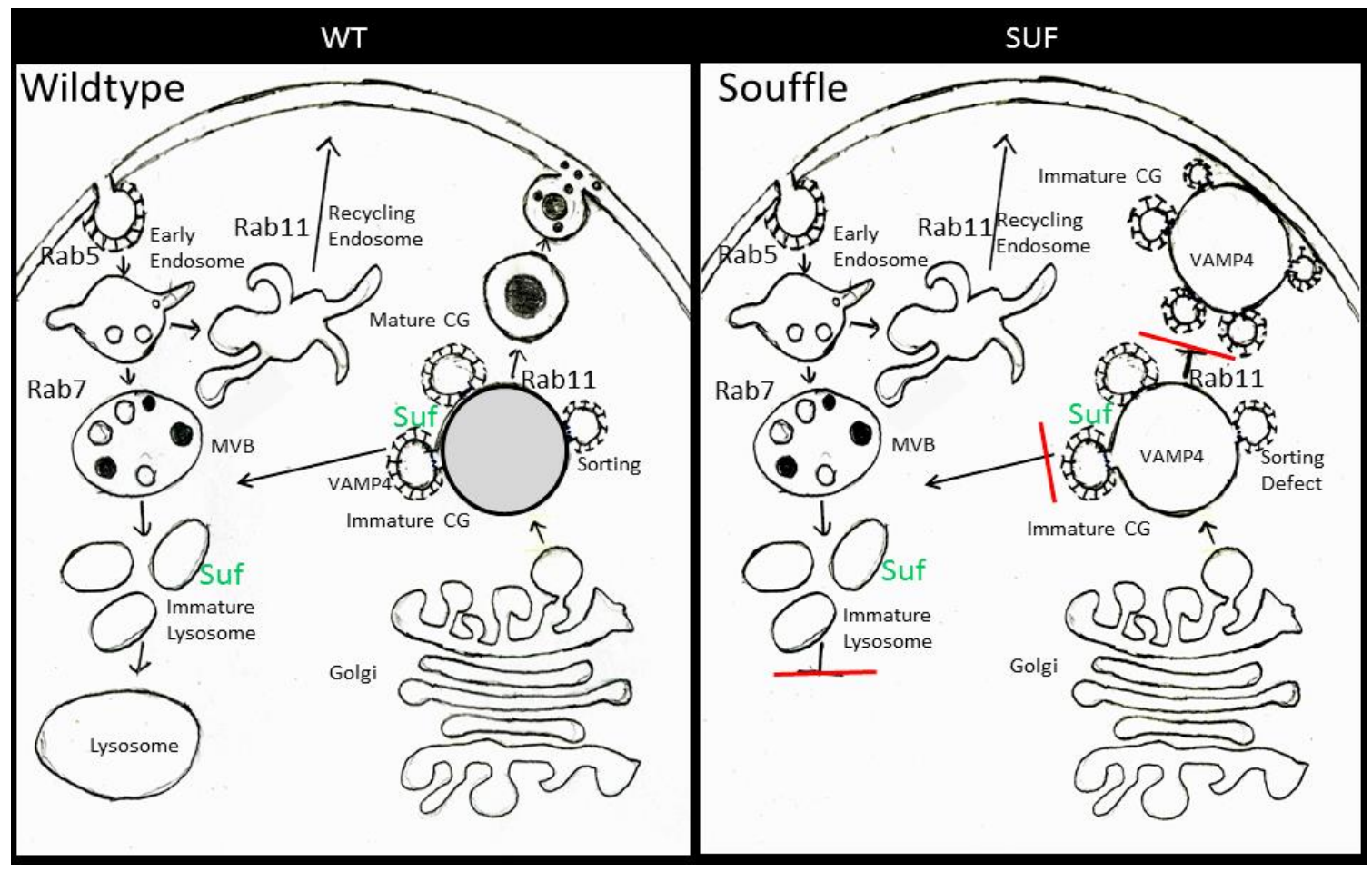

Figure 36. Soufflé regulates sorting and fission during secretory granule maturation.

a. Model for Soufflé (red) function on immature secretory granules (ISG). Suf/Spastizin is necessary for the abscission of Clathrin-coated buds. Whereas the compartment matured into secretory, cortical granules (CG) with a dense-core, the Clathrin-coated vesicles enter the endolysosomal transport route probably to lysosomes. b. The drawing illustrates the defect in the suf mutant and the role of souffle during secretory granule maturation, which leads to successful sorting into formation of dense-core vesicle. Left panel shows in the wild type and right side panel shows in the suf mutant oocytes.

\subsection{Role of soufflé in lysosomal biogenesis and function}

The Soufflé mutant was discovered in maternal a screen by its phenotype opaque egg instead of transparent eggs (Dosch et al.,2004). This defect suggested that the suf mutant has a defect in endo-lysosomal pathway, which fails to degrade yolk proteins in the oocyte, which creates the opaqueness. Surprisingly, we discovered an unknown role for suf in the secretory pathway but the question is still unanswered, why the suf oocytes are 
opaque. The defect in the lysosomal pathway is directly influenced by souffle mutation or is it an indirect consequence of the defect in the secretory pathway? (Fig. 37).

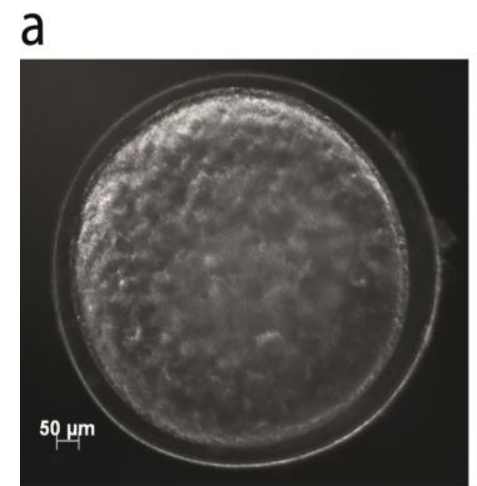

WT mature oocyte

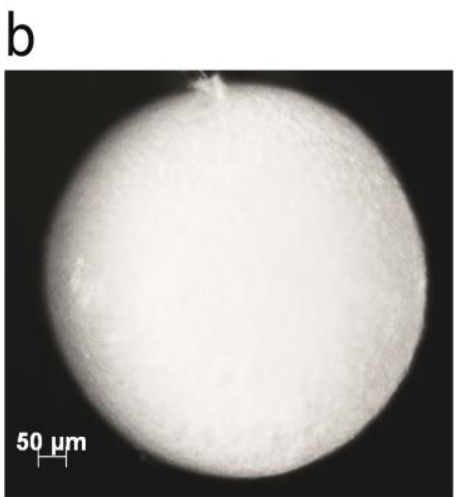

WT immature oocyte

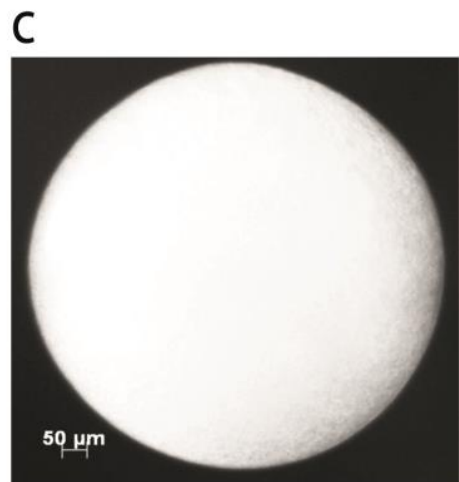

suf mutant oocyte

\section{Figure 37. Soufflé mutant lays opaque egg.}

a. light microscopy image of wt mature oocyte/egg. b. Image of wild-type immature oocyte with opaqueness. c. image of suf mutant mature oocyte/egg looking similar to wild-type immature oocyte. Scale $-50 \mu \mathrm{m}$.

\subsubsection{Suf mutant shows defect in yolk degradation}

As the suf mutant was discovered for their opaque phenotype and the defect in yolk degradation during oocyte maturation in zebrafish, yolk profiling revealed that the yolk protein is not processed in the suf mutant compared to the wild type (Dosch et al., 2004).

\subsubsection{Suf mutant shows defect in lysosomal maturation}

The maternally delivered yolk protein is taken up by the oocyte through endocytosis. The yolk proteins are processed/degraded in lysosomes. Endosomal compartment analysis showed that suf mutant accumulates smaller/fragmented lysosome (Fig.20). To further analyze the lysosomal defect, I used cryo-electron microscopy and the analysis showed that the suf mutant accumulates smaller lysosomes consistent with Immuno-staining (Fig.38 a-e).The quantification of the lysosomal size showed that the suf mutant lysosome is $3 \mu \mathrm{m}$ smaller than the wild type (Fig.38 f). These results indicate that suf is necessary for lysosomal maturation.

\section{Figure 38. Soufflé mutant shows defect in yolk degradation.}

a. Electron micrograph showing fragmented lysosome in suf mutant mature oocyte. b. High resolution image showing the presence of yolk crystal still in the suf mutant oocyte lysosomal structure which is absent in the wildtype. c. Image from immature oocyte 
showing the crystal yolk in wt and mutants and mutants accumulating small yolk vesicles. d. Showing magnified view of the yolk crystal from wt and suf mutant oocyte lysosome. e. Cryo-electron micrograph from wt and suf mutant oocytes showing fragmented lysosome, which are used for quantification of the lysosomal size. f. Bar diagram shows the mean size of the lysosome in wt and suf mutant oocytes. Notice the difference in the size of lysosome in the suf mutant compared to wildtype.

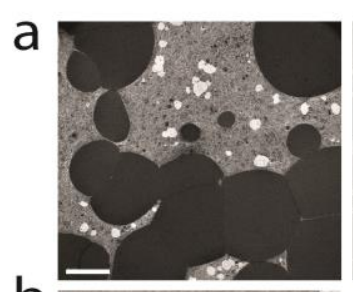

$\mathrm{b}$

$\mathrm{C}$
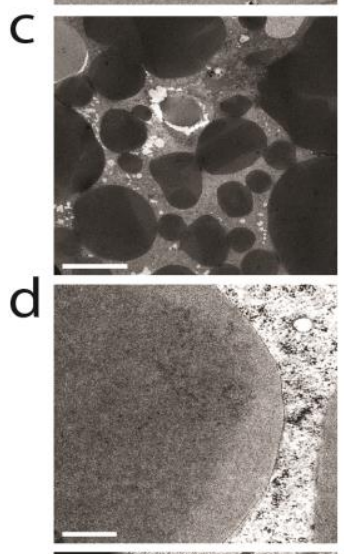

e

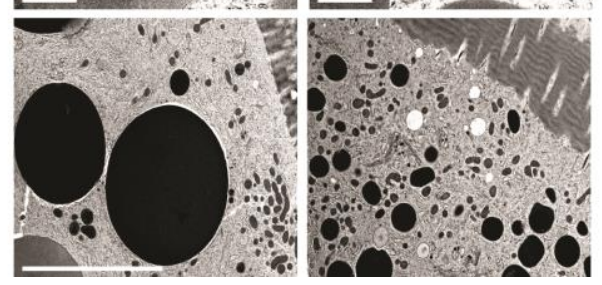

f

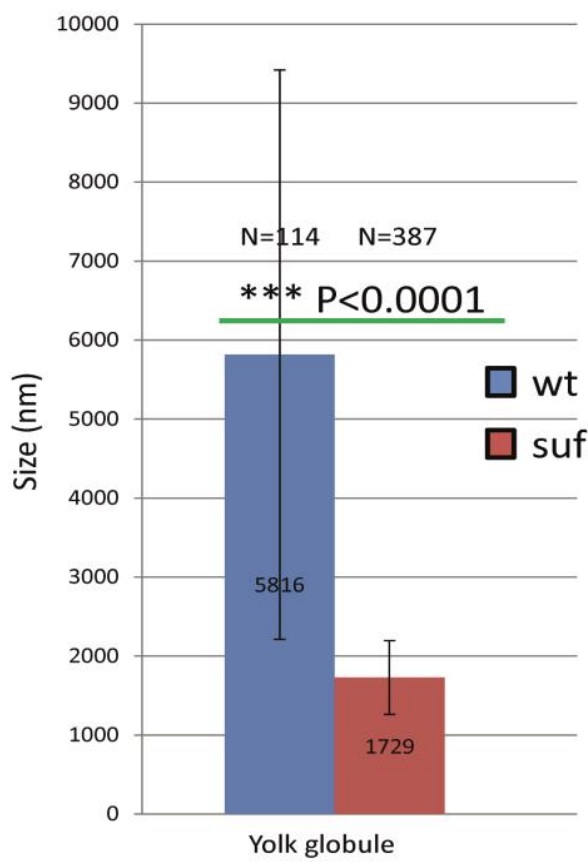

\subsection{Yolk endocytosis is not affected in suf mutant}

\subsubsection{Yolk reaches the lysosome}

Although the defect in lysosome maturation explains the opaque phenotype, but it still does not address whether the yolk endocytosis is functioning properly and has arrived in the smaller lysosome or not in the suf mutant. Immuno-staining for the yolk protein showed it reaches the lysosome in the suf mutant consistent with Rab5 and Rab7 immunostaining showing no defect (Fig.39a). 


\subsubsection{In-vivo endocytosis assay for LDL}

LDL follows the degradative transport route to lysosomes and the yolk-receptor belongs to the LDL-receptor superfamily (Goldstein et al.,1985; Schneider.1996). Adding fluorescent LDL to the culture medium labeled yolk globules in zebrafish oocytes, which correspond to lysosomes in somatic cells (Sire et al.,1994). However, we observed no difference in the LDL transport to wild type or suf mutant lysosome, suggesting that transport along the degradative pathway has not been disrupted (Fig.39b).
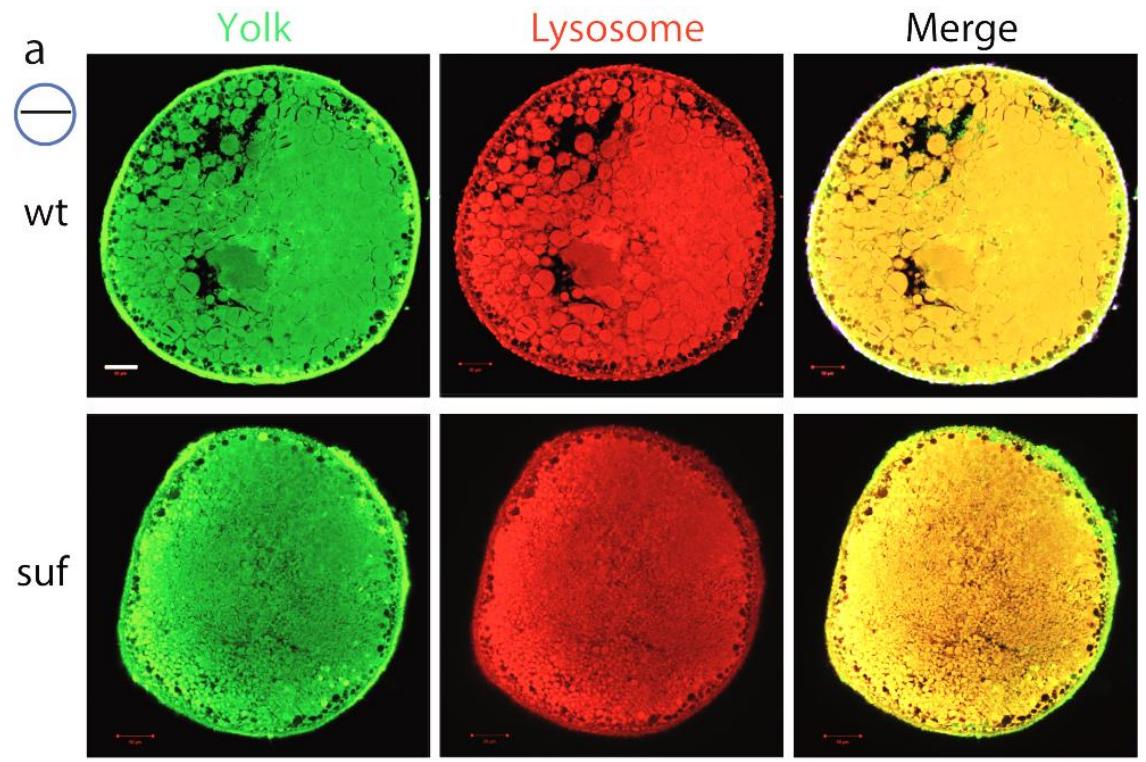

LDL
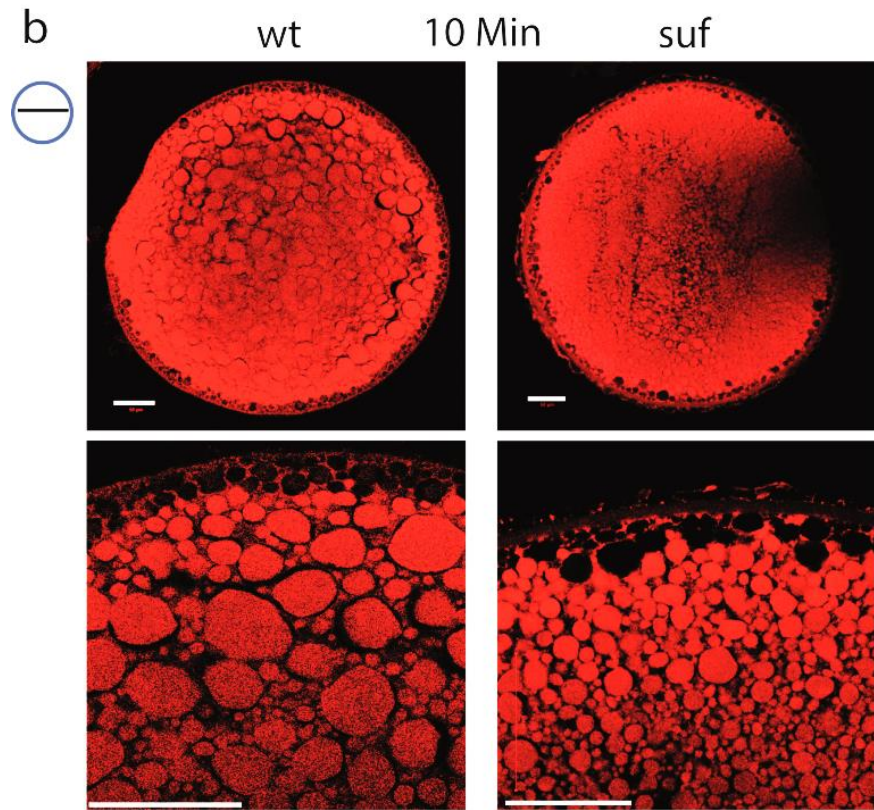

Figure 39. Yolk reaches the lysosome in suf mutant oocyte.

a. Immuno-staining for the yolk protein vitellogenin in green and lysosomes in red showing that in both wt and suf mutant the yolk reaches the lysosome. Top panel from wild type, 
bottom panel from suf mutant in separated individual channel, and merge. b. In-vivo endocytosis assay using LDL cargo in red shows that LDL reaches lysosome in the suf mutant like in the wild type after 10 mins of intake consistent with yolk staining. Small cartoon at the left top shows the optical section. Scale $-50 \mu \mathrm{m}$.

\subsection{Vitellogenin processing enzyme Cathepsin D is not affected in suf mutant}

Cathepsin D processes the vitellogenin into yolk proteins and then they are degraded by other group of Cathepsins (Cathepsin B, F and L) in the lysosome. If the yolk protein reaches the lysosome in the suf mutant, why the yolk proteins are not processed? Does the lysosome have all the proteolytic enzymes for the degradative function? To address the question, I wanted first to check the vitellogenin processing enzyme Cathepsin D. The immuno-staining showed that the Cathepsin D is present in the lysosome in the suf mutant (Fig.40) consistent with the presence of different yolk proteins in the suf mutants, suggesting that the lysosomal physiology is still suitable for the Cathepsin D activity.
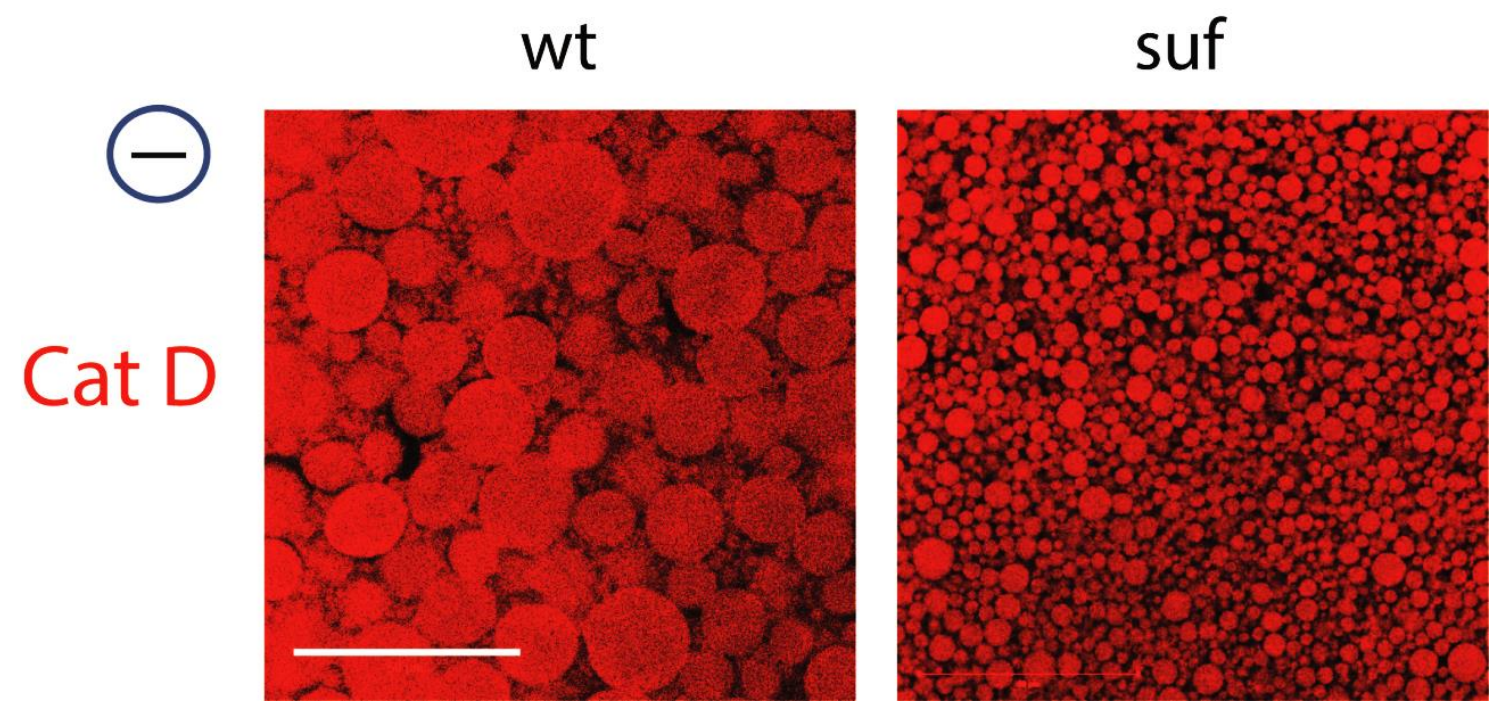

Figure 40. Cathepsin D protease reaches the lysosome in suf mutant oocyte.

Confocal image shows the presence of cathepsin D proteases in red in the lysosome of wt and suf mutant oocytes. Fragmented lysosomes are well visible in the suf mutant oocyte. Scale $-50 \mu \mathrm{m}$. Cartoon shows the optical section plane. 


\subsection{Yolk protein degrading cathepsins are not delivered to lysosomes in the suf mutant}

\subsubsection{Cathepsin B, F and $L$ not delivered to lysosome}

The yolk proteins are processed two times and he second processing is considered as a degradative process. The primary processing enzymes, Cathepsin D is present in the lysosome and processes the VTG into different yolk proteins. I wanted to check if the degrading enzymes are delivered to lysosome and used immuno-staining for three different Cathepsins. Interestingly, the staining showed that the degrading Cathepsins (Cathepsin B, $\mathrm{F}$ and L) are not delivered to the lysosome in the suf mutant (Fig. 41a \& b; Fig.42a). I also discovered that the Cathepsins were trapped in the secretory granules, suggesting that they are not sorted to lysosomal pathway. The amounts of enzymes are drastically reduced inside the oocyte that accumulated very strongly on outside membrane. To check if the reduction in the enzymes amount is because of less protein production or they are degraded or secreted out, I analyzed early oocytes before the yolk accumulation takes place (Stage Ib) and on staining it showed that the enzymes were present in the secretory granule similar to the wild-type oocytes (Fig.42b). These results prove that the degrading Cathepsins are not delivered to the lysosome and hence the yolk accumulates thus creates the opaqueness in the suf mutant. This also gives an indication that the lysosomal defect is a consequence of the sorting defect in the secretory granule.

Figure 41. Cathepsin $B$ and $L$ are not delivered to the lysosome in suf mutant oocyte. a. Double staining for cathepsin B in green and lysosomes in red in single channels and the merge at the right side. Top panel from wildtype and bottom panel from suf mutants. Notice that in the suf mutant absence of cathepsin B in the lysosome and accumulation in the secretory granule, which are negative for the lysosome staining in the suf mutant only. b. Double staining for cathepsin L in green and lysosome in red showing the absence of cathepsin L in the suf mutant oocyte lysosome like cathepsin B. Top panel from wt and bottom panel from suf mutants. Scale $-50 \mu \mathrm{m}$. 

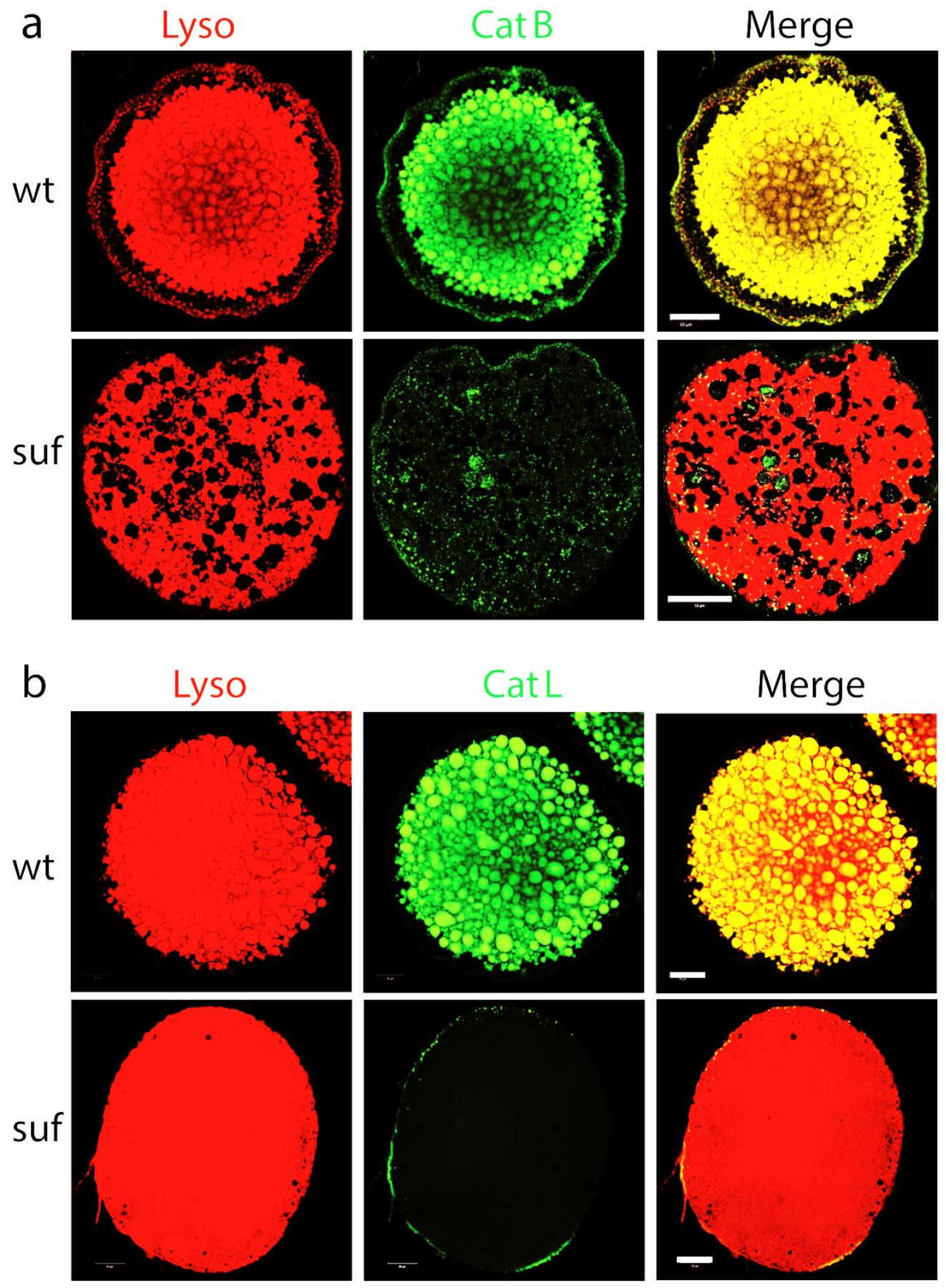


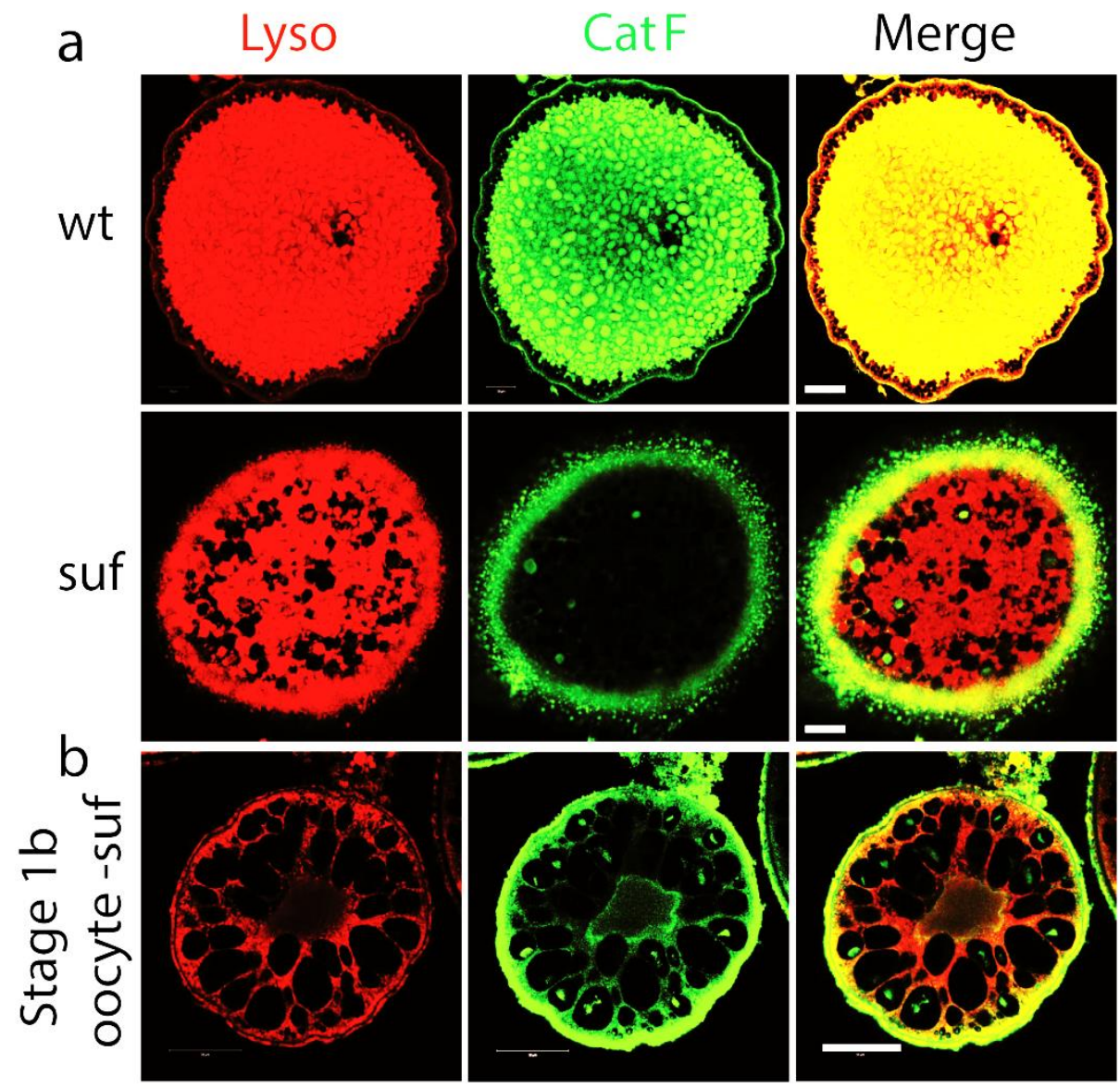

Figure 42. Cathepsin $\mathrm{F}$ is not delivered to the lysosome in suf mutant oocyte.

a. Similar to cathepsin B and L cathepsin F also fails to reach the lysosome in the suf mutant. Cathepsin $\mathrm{F}$ in green and lysosome in red in single channels and merge at the right side. Top panel from wt and bottom panel from suf mutants. Notice the accumulation of cathepsin $\mathrm{f}$ in the secretory granule in $s u f$ mutant similar to cathepsin B. b. Stage $1 \mathrm{~b}$ oocytes before vitellogenesis from suf mutants shows normal distribution of Cathepsin $\mathrm{F}$ like wild type. Notice the cathepsin F in green in the secretory granule before yolk arrival in the lysosome. Scale $-50 \mu \mathrm{m}$.

\subsection{Lysosomal receptors M6PR and Sortilin transported correctly}

Non-delivered lysosomal enzymes are trapped in the secretory granule indicating that the transport from secretory pathway to lysosomes is defective in suf mutant. This result also confirms that the lysosomal defect in suf mutant is a consequence of sorting defect in the secretory granule during maturation. The lysosomal cargos are transported from secretory granule through receptor mediated sorting and vesicle fission (Fig.43a). Two of the well-studied and most used pathways for lysosomal sorting is Mannose-6Phosphate receptor (M6PR) and Sortilin. These two receptors are known to sort different 
cathepsins in different cell types. To check if these receptors are sorted to lysosome in the suf mutant, I looked for these receptors in the mutant oocyte using antibody staining and the analysis revealed that both the receptors reached lysosome in the suf mutant oocyte (Fig.43 b \& c). Consistent with the presence of Cathepsin D in the suf mutant lysosome, thus suggesting that suf might regulate pathway independent of these receptors.

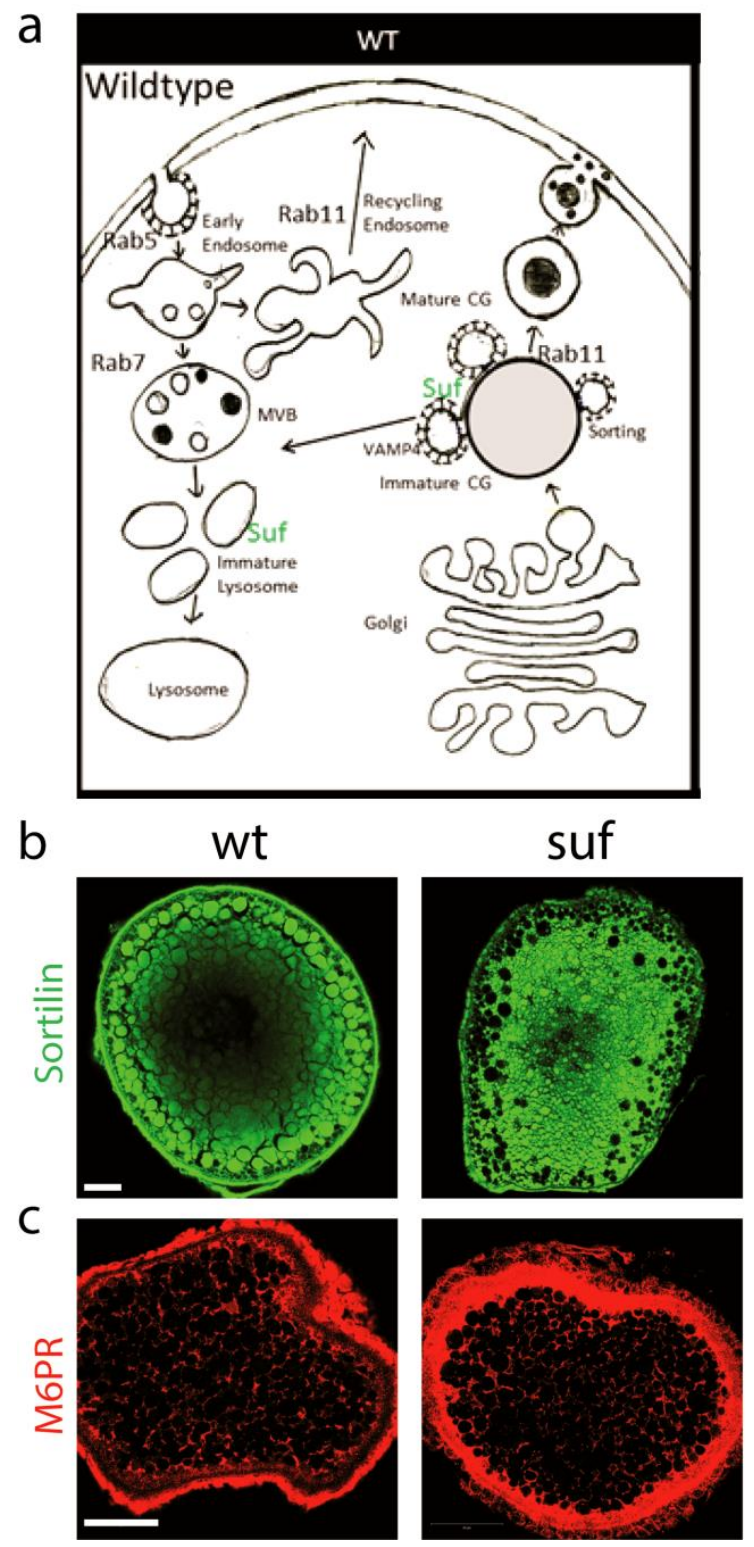

Figure 43. M6PR and Sortilin reaches endo-lysosomal system

a. Cartoon shows the connection between secretory pathway and endosomal system from immature secretory granule. Budding vesicle from immature secretory granule delivered to the endo-lysosomal system through receptors mediated sorting. b. Immuno-staining for sortilin in green shows the presence of sortilin in lysosomal system in suf mutant similar to 
wild type. c. Staining for M6PR in red shows the presence the receptor in endo-lysosomal system like wild type. Left panel from wt and right panel from suf mutant. Scale - $50 \mu \mathrm{m}$.

\subsection{Endo-lysosomal sorting adaptor protein complexes (AP1 \&GGA1)}

The sorting process consists of three different functions and they are connected. First, the cargo binds to its receptor followed by finding the right adaptor protein complex and the coat proteins (Fig.44 a). Since, the receptors reach lysosome in the suf mutant oocytes and suf was discovered to interact with newly discovered adaptor protein complex 5 (AP5), I wanted to analyze if the other adaptors are known to involve in sorting cargos to the endo-lysosomal system are, also properly sorted. Immuno-staining for both adaptors (AP1G1 \& GGA1) showed no difference compared to the wild type (Fig.44 b), suggesting that the regular M6PR dependent lysosomal pathway is not affected in the suf mutant oocytes.

Figure 44. Adaptor protein complexes AP1 and GGA1.

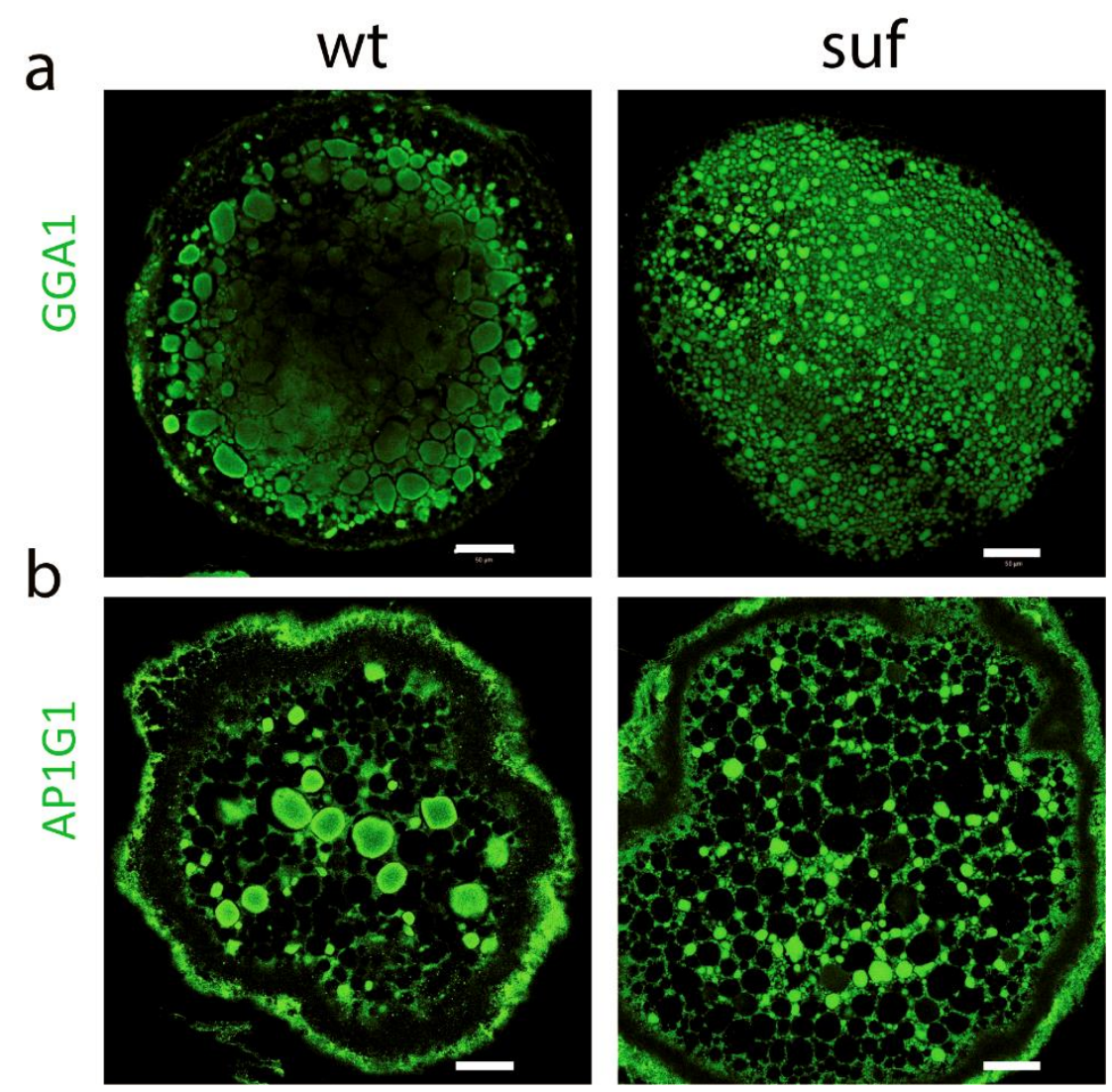

\subsection{Lysosomal membrane proteins (LAMP1 and V-ATPase)}

Lysosome function depends on membrane proteins and soluble proteins. The membrane proteins give the integrity and protect the cellular organelles from the acidic lysosome. To investigate if the membrane proteins are intact and functional in the suf 
mutant lysosome, I investigated LAMP1 and proton pump v-ATPase d1 subunit. The LAMP1 reaches the lysosome in the mutant but the LAMP1 positive intermediate from secretory pathway is reduced (Fig.45a, b \& c). Surprisingly, v-ATPase subunit d1 was not found inside the oocyte and strongly accumulated outside on the membrane similar to Cathepsins, suggesting that the particular subunit is not delivered to lysosome (Fig.46). The reduction in the LAMP1 positive vesicles suggests that suf mutant might possess a defect in LAMP carrier pathway (Pols et al.,2013), which was recently discovered.

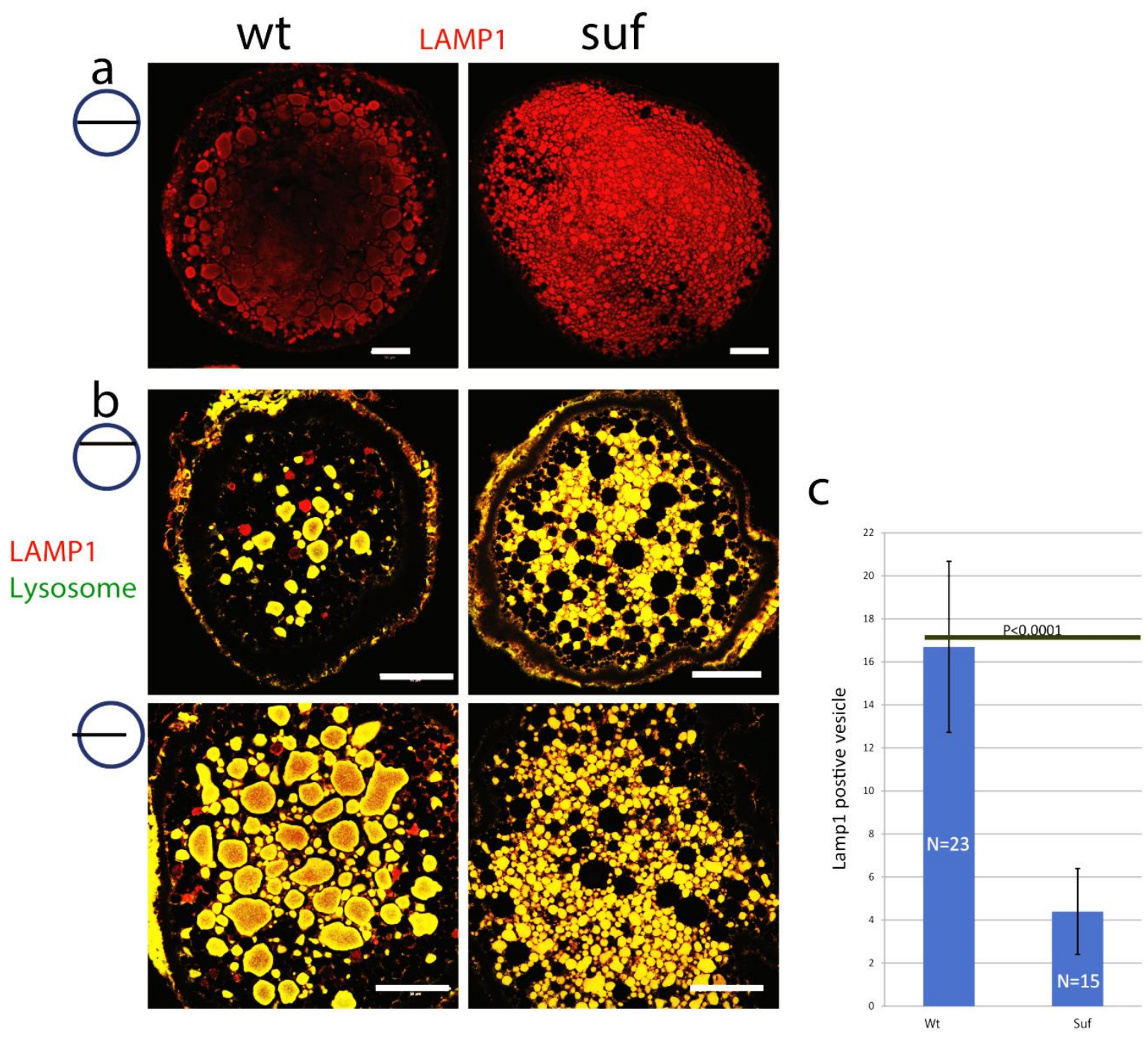

Figure 45. LAMP carriers are reduced in suf mutant oocytes.

a. Confocal image shows the LAMP1 in red reaches the lysosome in wt and suf mutant. b. But the LAMP carrier intermediates derived from secretory granules are severely reduced which are LAMP1 positive in red but not positive for lysosome staining (in green). Bottom panels shows different focal planes of the oocyte. Left panel from wt and right panel from suf mutants. Scale $-50-\mu$ m. c. Bar diagram shows the mean number of LAPM carrier 
intermediates from wt and suf mutant oocytes counted using confocal images. $\mathrm{p}$ value shows the significant difference. Error bar indicates the standard deviation.
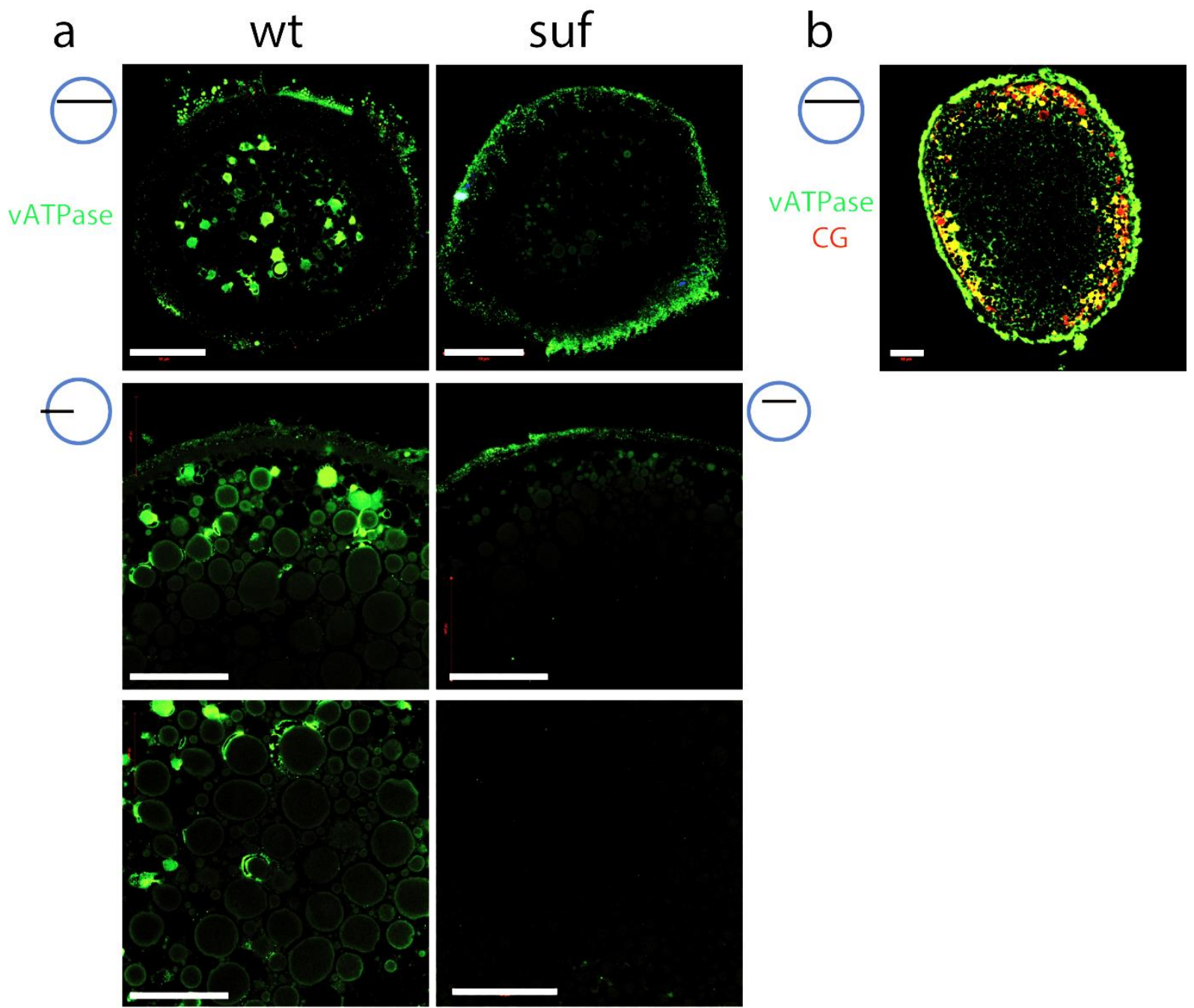

\section{Figure 46. V-ATPase subunit d1 does not arrive in suf mutant lysosome.}

a. Image showing V-atpase subunit $\mathrm{d} 1$ in green in wt and suf mutant oocytes. Notice the absence of d1 subunit in the lysosome of suf mutant oocyte. Left panel from wt and right panel from suf mutant. b. Double staining for $\mathrm{V}$-atpase in green and secretory granule in red showing co-localization of both on secretory granule in wt oocytes. Cartoon shows the optical section. Scale $-50 \mu \mathrm{m}$.

\subsection{LAMP carrier pathway is affected in suf mutant}

Commonly used receptor pathways did not show any defect and reduction in LAMP1 positive vesicles raising the question if VPS41 mediated Lamp carrier pathway is affected in the suf mutant. VPS41, a subunit of vacuolar/lysosomal homotypic fusion and vacuole protein sorting (HOPS) tethering complex originally discovered for its role in 
lysosomal fusion with different compartments. HOPS deficit yeast and other model organisms have fragmented lysosome, but VPS41 was discovered to have role in sorting lysosomal cargo with AP3 adaptor protein complex acting as a coat protein apart from its role in lysosomal fusion (Asensio et al.,2013). To investigate if VPS41 and AP3 were sorted properly, I did an immuno-staining for both VPS41 and AP3M2. Interestingly, suf mutant oocyte show much reduced VPS41 positive intermediate vesicles similar like LAMP1 positive vesicles (Fig.47 a \& b). But, AP3 did not show much difference or change in AP3 positive vesicle number (Fig.48 a \& b). These results shows that the suf mutant has a defect in VPS41 mediated LAMP carrier pathway to lysosome.

a
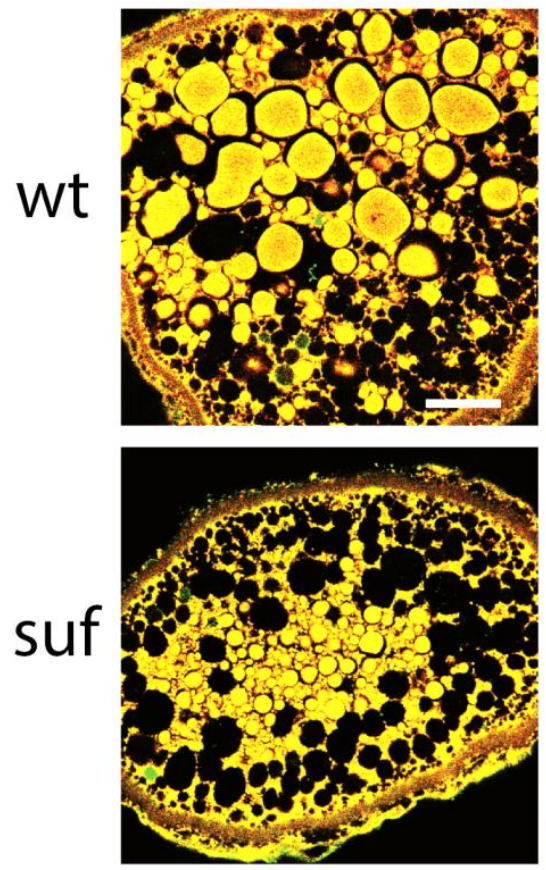

\section{Lysosome}

VPS41
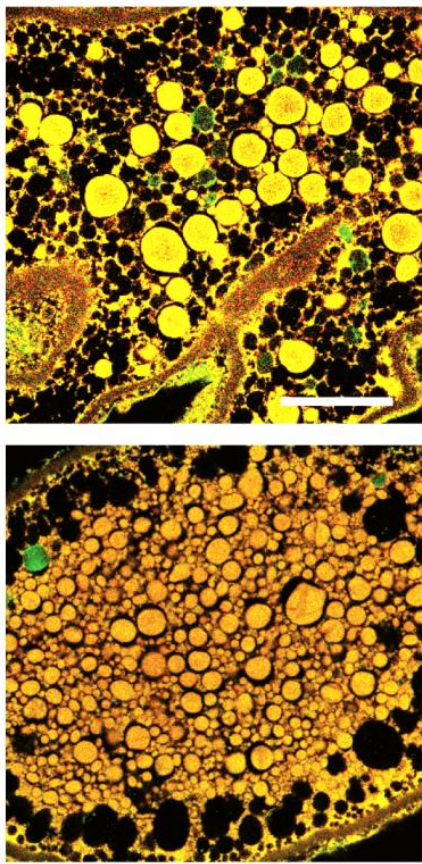
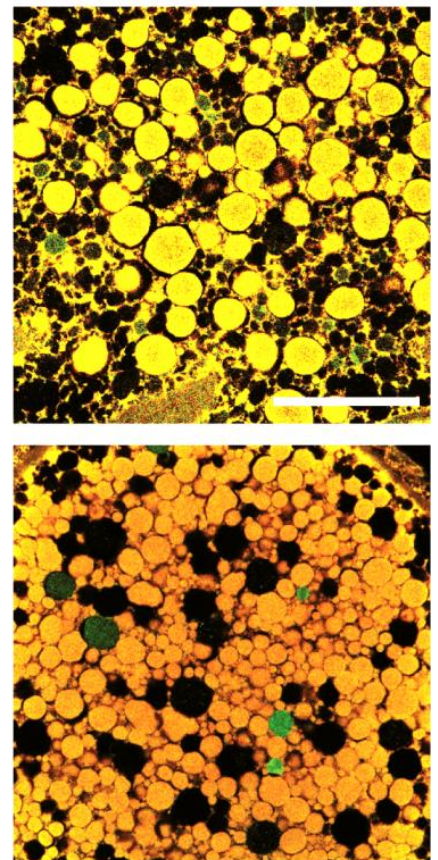

b

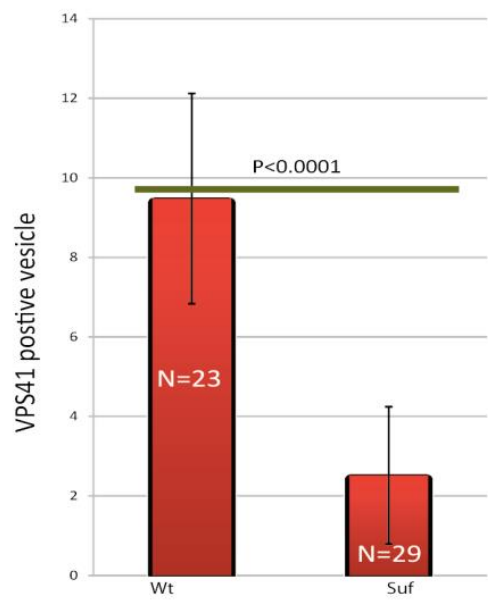


Figure 47. VPS41 positive Lamp carriers are reduced in the suf mutant.

a. immune-staining for VPS41 in green and lysosome in red in wt and suf mutant oocytes.

Top panel from wt and bottom panel from suf mutants. Notice the reduction of green positive vesicles in the suf mutant similar to LAMP carrier. b. Diagram shows the quantification of VPS41 positive vesicles only from wt and suf mutant oocytes. Bar shows the significant reduction in the number of VPS41 positive vesicles. P value shows the significance and error bar indicates the standard deviation. Scale $-50 \mu \mathrm{m}$.
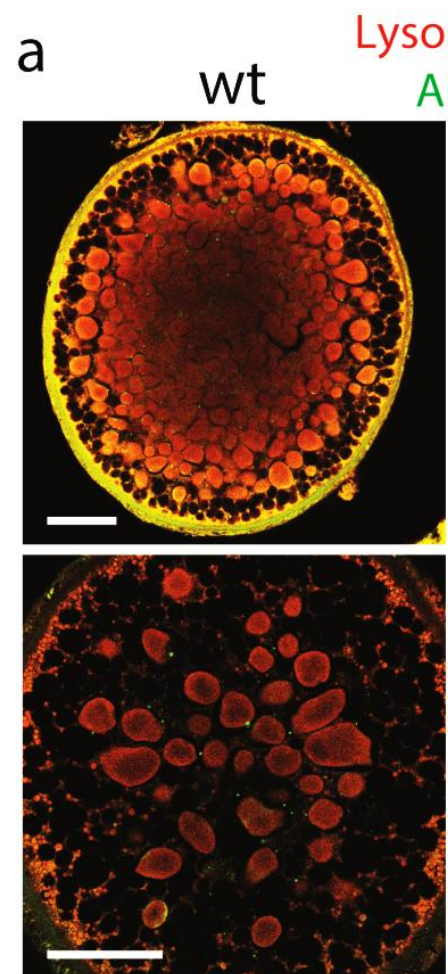

\section{AP3}
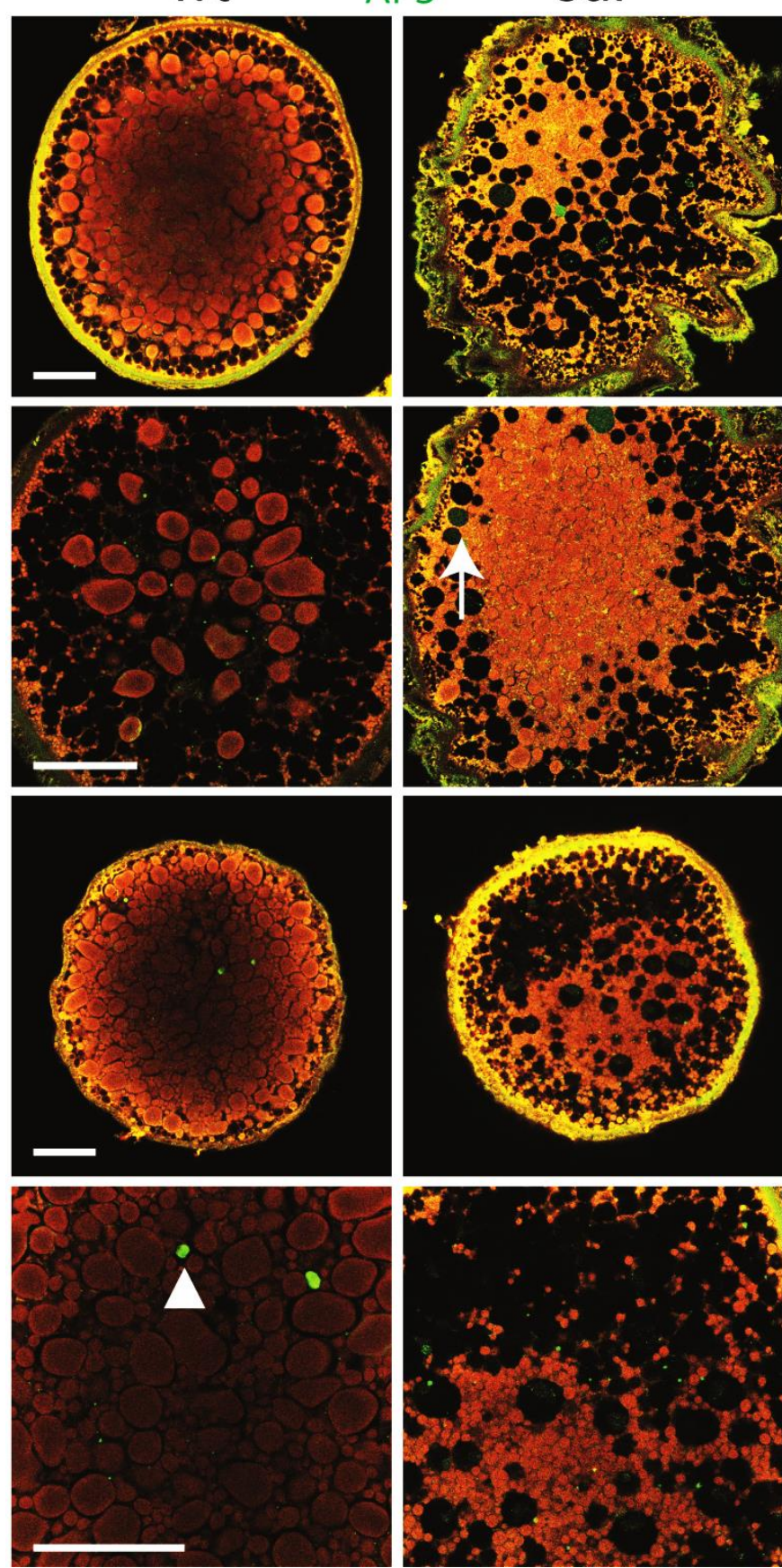
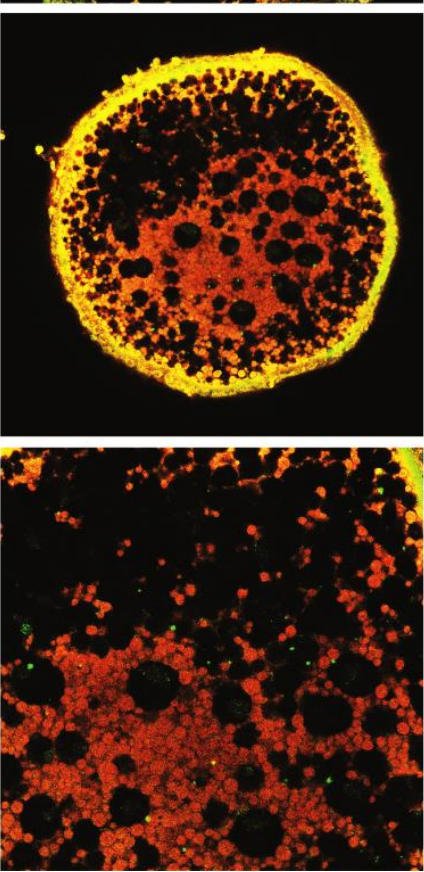

b

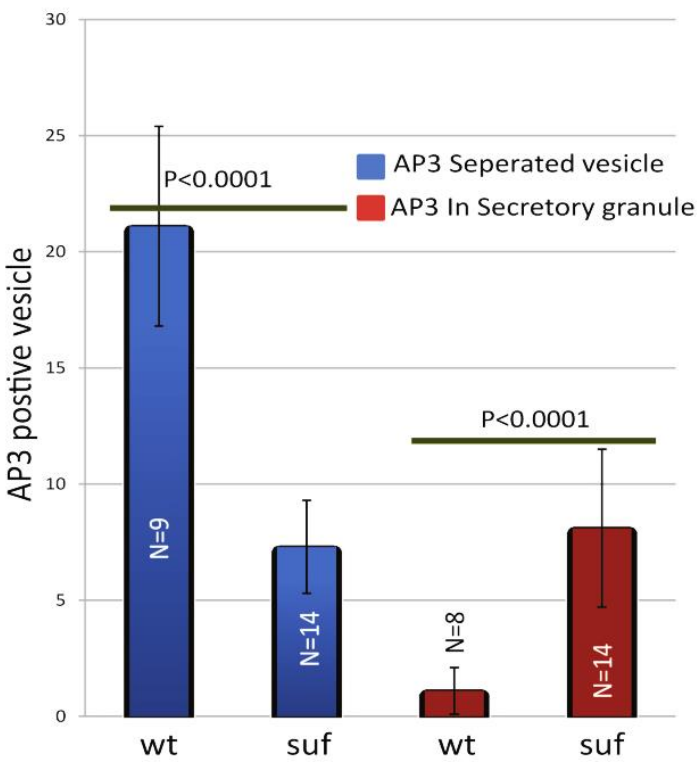


Figure 48. AP3 accumulates in secretory granule in suf mutant.

a. The image shows AP3 in green and lysosomes in red. Left panel from wt and right panel from suf mutants. Arrow indicates AP3 presence in secretory granule and white arrowhead indicates separated small AP3 positive vesicles in different focal plane of the oocyte. Scale $-50 \mu \mathrm{m}$. b. Diagram shows the quantification AP3 positive secretory granule in red bars and separated individual AP3 vesicle in blue bars. The red bar shows an increase in AP3 positive secretory granule in suf mutant compared to wt and blue bar shows the reduction in the separated individual AP3 positive vesicle in suf mutant. P value shows the significance and error bar indicates standard deviation.

\subsection{Blocking secretion causes opaque egg phenotype}

The further experiment on the lysosomal pathway confirmed that the defect is a consequence of secretory granule maturation defect. To check in-vivo whether the blocking of chorion elevation in zebrafish egg will show a lysosomal defect, the wild type eggs were treated with dynasore and control. The Dynasore treated wild-type eggs and secretion delayed by Hanks buffer both showed no chorion elevation (Fig.31a) and surprisingly, these eggs also showed opaque egg phenotype like suf mutant eggs (Fig.49a). If this opaqueness of the egg is because of the defect caused by the block in the secretion, I analyzed the yolk degradation profile in the treated eggs and yolk profiling confirmed that there is accumulation of bigger yolk protein in the secretion blocked sample (Fig.49b). This bigger yolk protein normally presents in the immature oocyte and they disappear during maturation by degradation but when we block the secretion, the egg starts accumulating this particular yolk protein, which corresponds well with their opaqueness and duration of treatments. This in-vivo functional experiment confirms that the lysosomal defect is indeed the consequence of secretory granule maturation defect.

\section{Figure 49. Delaying secretion inhibits lysosomal function.}

a. Image of the wt egg shows the opaqueness after delay in the chorion elevation process by dynasore treatment. First panel from wt mock treated and second from wt egg dynasore treated for 30 mins then activated for 5 mins and third panel from wt eggs not activated for 30 mins. Notice the failure of chorion elevation in both dynasore treated and chorion elevation delayed by Hank's buffer for 30 mins and also showing opaqueness like suf mutant oocytes. b. SDS gel for yolk profiling shows protein pattern in the oocyte, egg before and after activation followed by dynasore treated eggs of wt. Red rectangle box 
showing the reappearance a particular band at the top indicated by arrow similar to oocyte, which disappear in the egg.

a
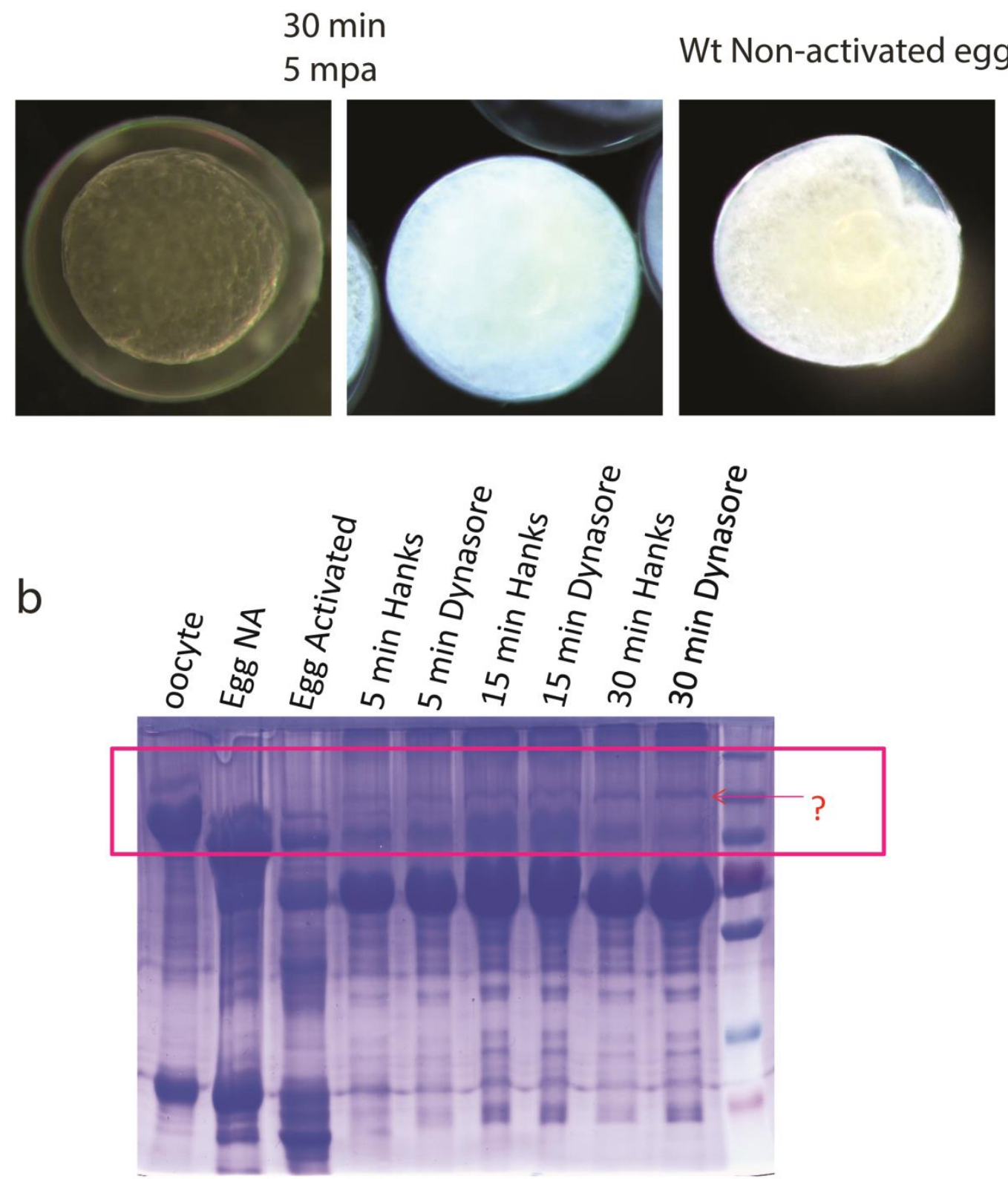

\subsection{Bioinformatics analysis of Soufflé and VPS41 protein}

The defect in the Lamp carrier pathway shows that suf might play role in VPS41 mediated lysosomal pathway. VPS41 deficit leads to fragmented lysosome and fails to form a dense-core vesicle with a defect in sorting of lysosomal cargos from the secretory granule (Asensio et al.,2013). This similar phenotype of VPS41 like suf mutant phenotype in zebrafish oocyte support the role of Soufflé in VPS41 mediated sorting. VPS41 has been shown to form Clathrin like coat with Clathrin Heavy Chain (CHC) domain (Fig. 50a) and 
Coatomer $\beta$ sub unit. To understand if Soufflé plays a similar role like VPS41 as a coat protein, I performed bioinformatics analysis for both the proteins. Analysis predicted both CHC and beta- Coatomer sub unit in multiple places of Soufflé protein (Fig. 50b, Appendix $4 \& 5$ ) consistent with the recent publication predicting that suf might play as coat protein with its solenoid like structure (Hirst et al., 2013). Moreover, Suf protein contains mostly a helical structure suggesting that this might provide as big scaffold protein (Fig. $50 \mathrm{c}$ ). The two different phenotype of suf mutant in lysosome and secretory granule maturation into dense core vesicle and their bioinformatics analysis further supports the novel role for Soufflé in VPS41 mediated lysosomal transport as a coat protein or a similar role like VPS41. Also for the first time we provide evidence that the defect in secretion causing the lysosomal defect which is mot observed phenotype in many neuro-degeneration diseases.

\section{Figure 50. Suf protein predicted to have Coat protein sub unit and CHC like VPS41.}

a \& b shows the predicted protein domain in VPS41 and soufflé with their corresponding region. Prediction shows that suf protein also has clathrin heavy chain homology and $\beta$ subunit of coat protein similar to VPS41. c. Graph shows the predicted secondary structure of the suf protein. The helices in red and sheets in green. Bottom panel shows the probability of forming coiled -coil structure. Suf protein shows the possibility of being a scaffold protein with its lot of helical structure.

\subsection{Motor neuron analysis in suf mutant}

The soufflé gene is conserved in all vertebrates and expresses in all tissues. The soufflé mutation (p96re) causes specific phenotype during oogenesis in zebrafish, but similar mutation (c.6702_6771 del) in this gene in human cause's progressive motor neuron degeneration called Hereditary Spastic Paraplegia (HSP). This tissue specific phenotype and late appearance creates difficulty to study the disease and to create models. Since we study the function of the protein in the oocyte where we see the phenotype, I wanted to study if suf mutant zebrafish larva or adult show any defect in movement or any motor neuron developmental defect (Nora Crascanta, IMPRS masters in molecular biology, Lab rotation project). Morphologically the larva or adult homozygous fish did not show any difference to its heterozygous siblings or wild type. Analysis of the motor neuron and muscle protein showed no difference compared to the heterozygous siblings (Fig. 51). This result confirmed that the suf p96re mutation causes a specific phenotype during oogenesis and does not show any other defect during development in any other tissues, suggesting that the suf mutant oocyte provides fitting model to study the Soufflé function. 
a

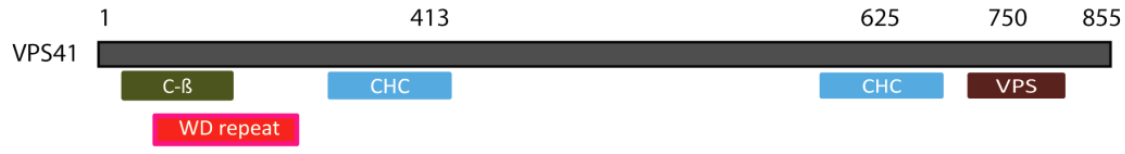

b
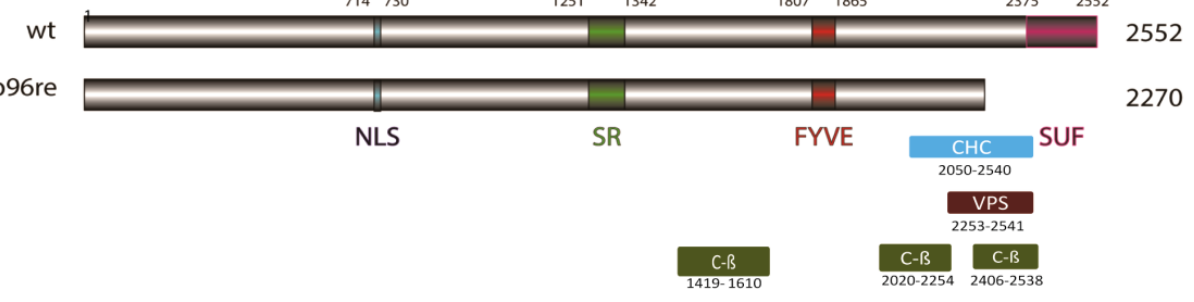

C SUF protein

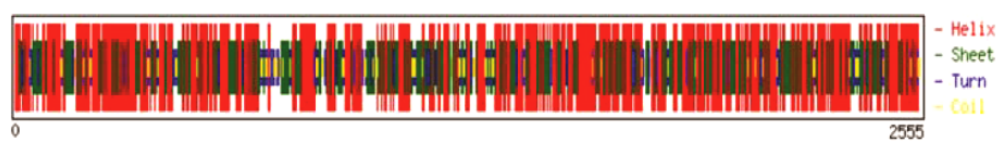

Helical structure

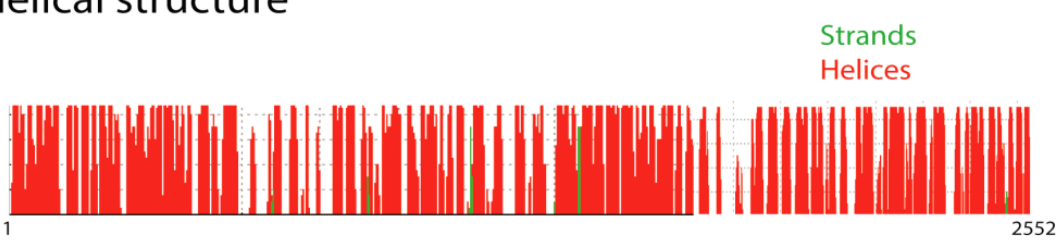

Coiled Coil Probability
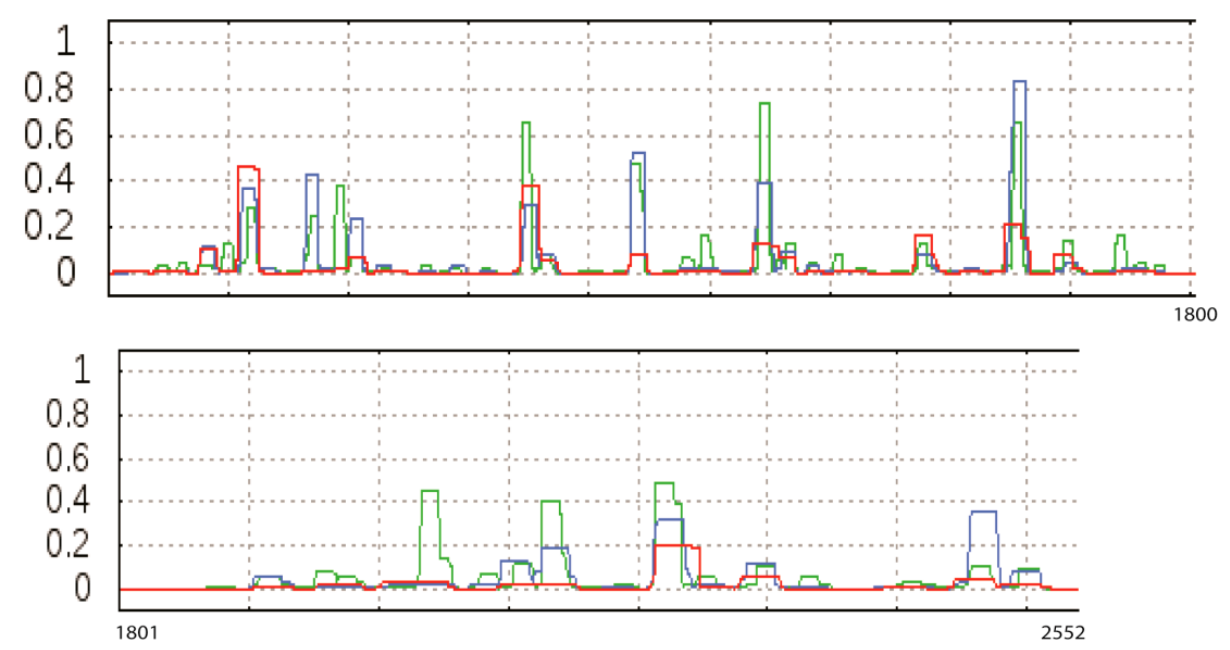
a

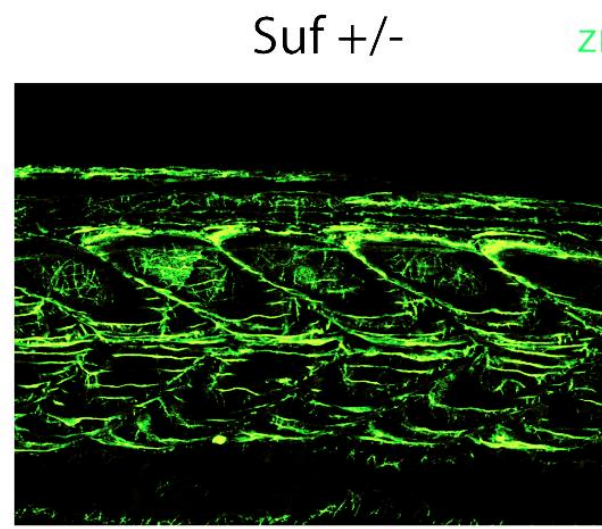

$\mathrm{zn}-8$

Suf -/-
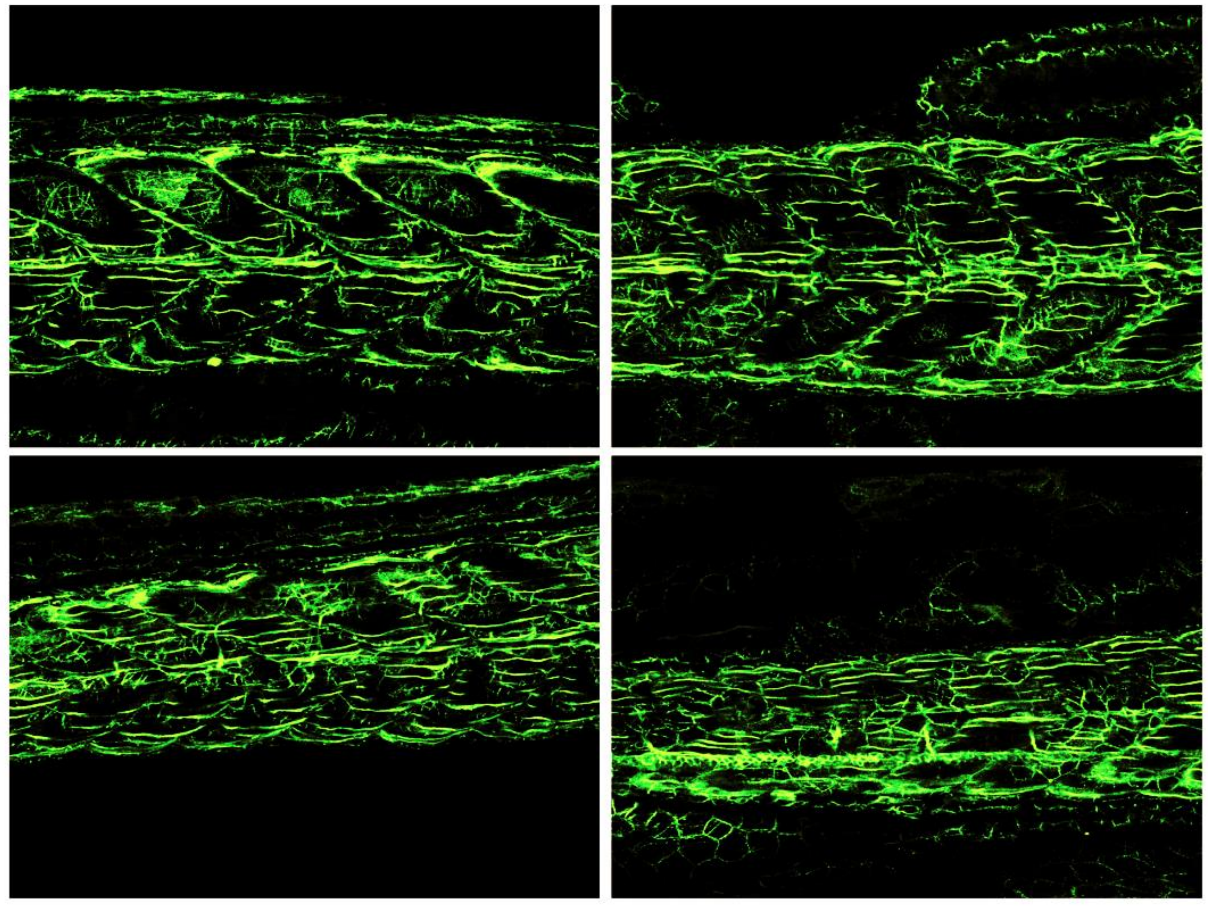

$\mathrm{b}$

$$
\text { Suf +/- }
$$

Suf -/-
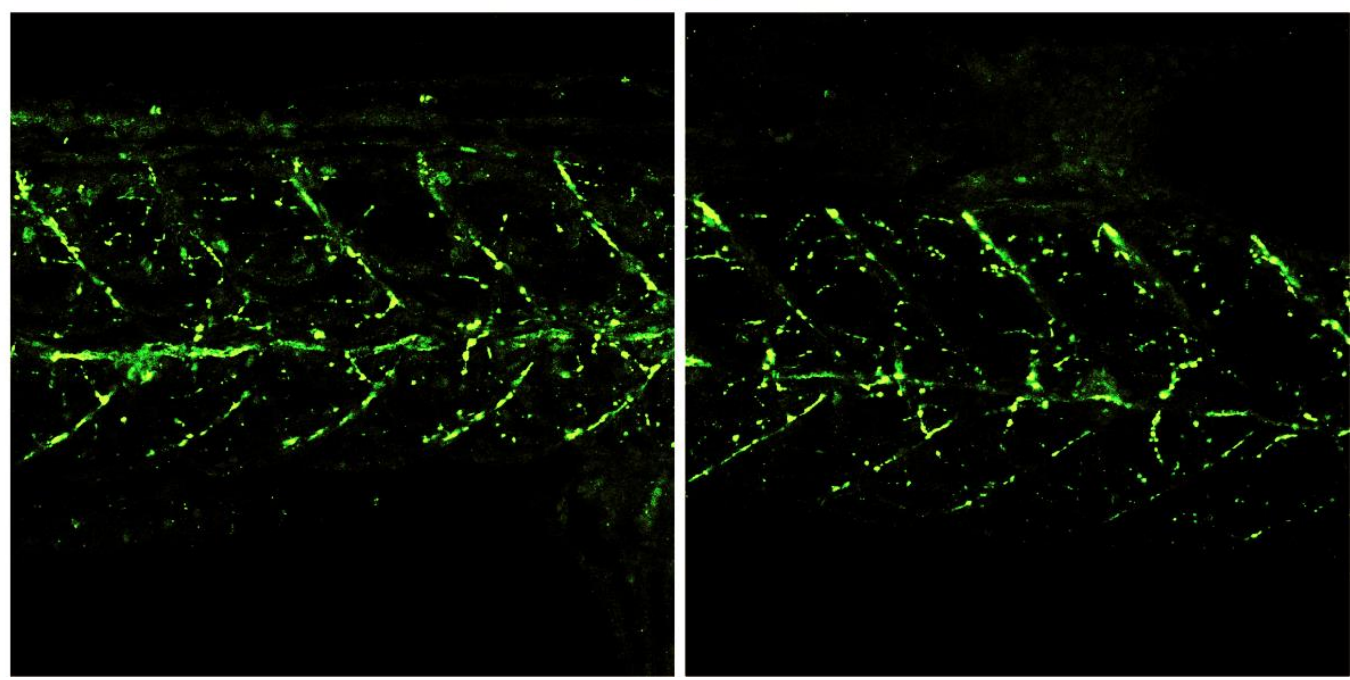

Figure 51. Motor neuron analysis of suf mutant fish.

a. Antibody staining for zn- 8 on 3 day old fish. $\mathrm{Zn}-8$ in green and the picture shows the trunk region of the spinal. The head is on the left side and tail is on the right of the image. Left panel is from suf heterozygous and right panel is from suf homozygous fish. b. Staining for SV-2 in suf hetero and homozygous mutant fish. SV-2 in green and the picture shows the trunk region of the spinal. The head is on right and tail is on left side. Both staining showed no difference in the motor neuron. 


\subsection{Suf protein knockdown by morpholino shows defect in notochord}

\section{formation}

Since the suf p96re allele is a hypo morph, I wanted to see if full protein gets knock down on using a morpholino reported (Martin et al., 2012). It is also to check any additional defect during early development. The knockdown showed 50\% twisted tail phenotype, $30 \%$ normally developed animal (Fig. 52 a, b \& c). Further analysis for motility did not show any defect in the normally developed animal and immuno-staining for motor neuron marker also showed no defect in development, but interestingly the twisted tail animals showed enlarged vacuoles/Lysosome structure in the notochord (Fig. $52 \mathrm{~d})$. The serially arranged lysosomes in the notochord are known to play essential role in spinal development. This result suggests that the suf might have a role during notochord development in the zebrafish.

\section{Figure 52. Suf morpholino knockdown shows defect in notochord lysosome.}

a. Morphological image of wt embryo injected with a control morpholino. b. Images show the three different phenotype of the wt embryo injected with the suf morpholino. c. Quantification of the suf knockdown phenotype. Around $30 \%$ shows normal development, around $42 \%$ shows twisted tail phenotype and $15 \%$ embryos not hatched. d. Immunostaining for ZNP-1 on control morpholino and suf morpholino injected fish. ZNP-1 in green shows no difference in motor neuron in normal developed suf morphant but the twisted tail fishes show that their lysosomal size is enlarged compared to the control morphant. 

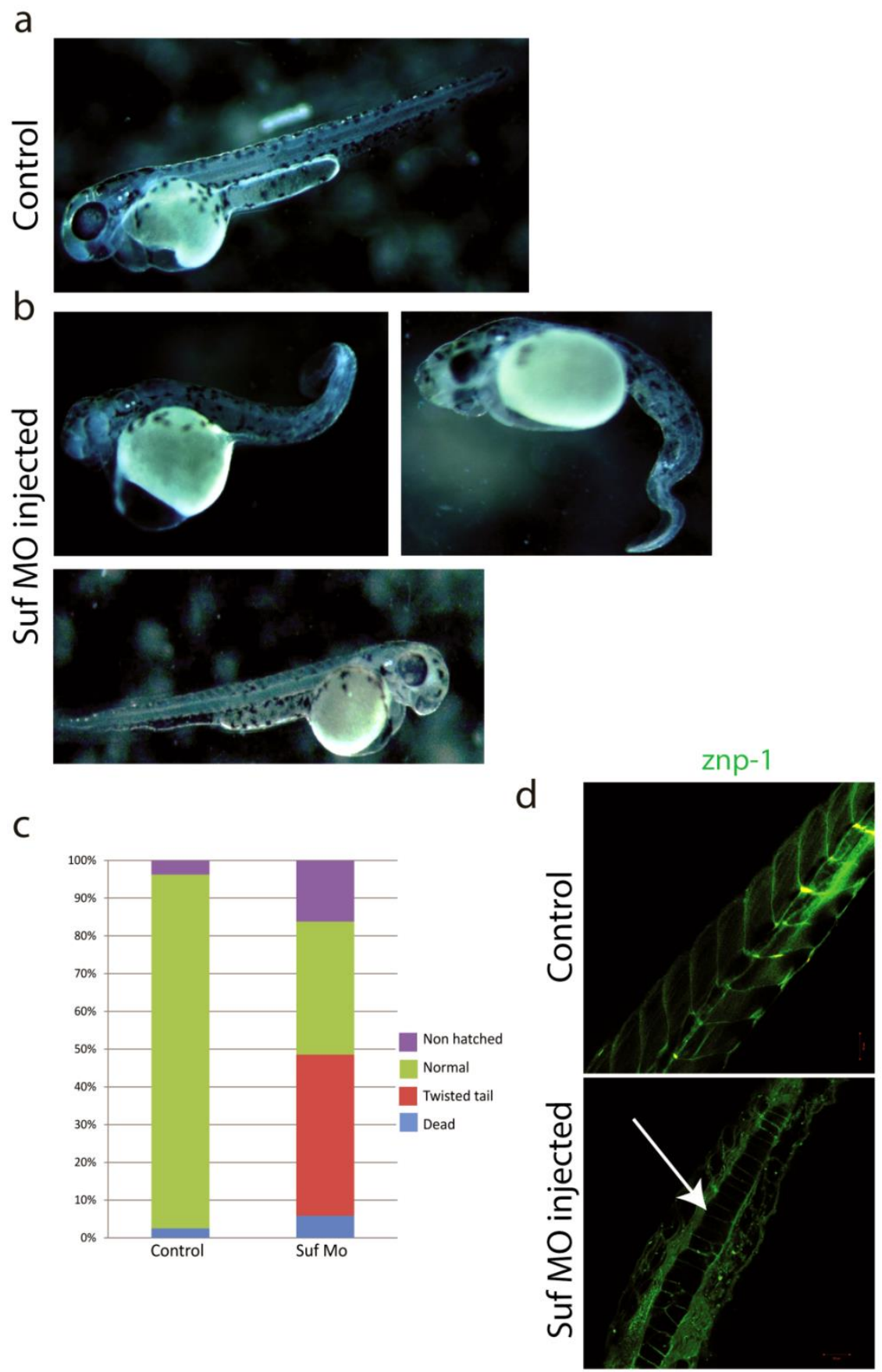

\subsection{Analysis of SPG15 mutant patients fibroblast}

Consistent with our results in zebrafish oocyte, the mutation in human does not cause any defect in any other tissues but motor neuron. To analyze further if the human SP15 patient fibroblast (c.6702_6771 del) showed a similar defect which I discovered in the suf mutant oocyte, we stained for several marker proteins and did live imaging for endocytosis, vesicle fission and other vesicle trafficking proteins (Susanne Schlick, IMPRS masters in molecular biology, Lab rotation project). The analysis showed no defect in any of the analyzed processes, which had showed a defect in the suf mutant oocytes (Fig. 53), 
consistent with earlier reports and disease pathology. These results from fibroblast and motor neuron analysis shows that the specific defect in the oocyte is similar to the motor neuron defect in humans, suggesting a similarity or high sensitivity of the zebrafish oocyte and the motor neuron for suf p96re mutation. Hence, the Soufflé mutant provides an excellent experimental model to understand this tissue specific disease phenotype and molecular mechanism.

\section{Figure 53. Analysis of spg15/spastizin mutant fibroblast cells.}

a. Confocal image of antibody staining for cellular trafficking protein marker involving endocytosis (Rab5, Rab7 and Rab11 in green and nuclei in blue). TGN38 in green as a marker for Golgi secretion and LAMP1 in green as marker for lysosome. SPG15 human antibody and zSuf antibody against zebrafish suf protein. Left panel is from wt cells and right side is from spg15 mutant cells. b. In-vivo analysis of endocytosis, secretion, lipid raft and recycling using fluorescent cargos. LDL in red shows no defect in endocytosis, $\mathrm{tfn}$ in red shows no difference in recycling, $\mathrm{CTxB}-$ Cholera toxin $\mathrm{B}$ in red shows no difference in lipid raft and Lectin in green shows no difference in secretion. Left panel is from wt cells and right side panel from spg 15 mutant cells. 
Results

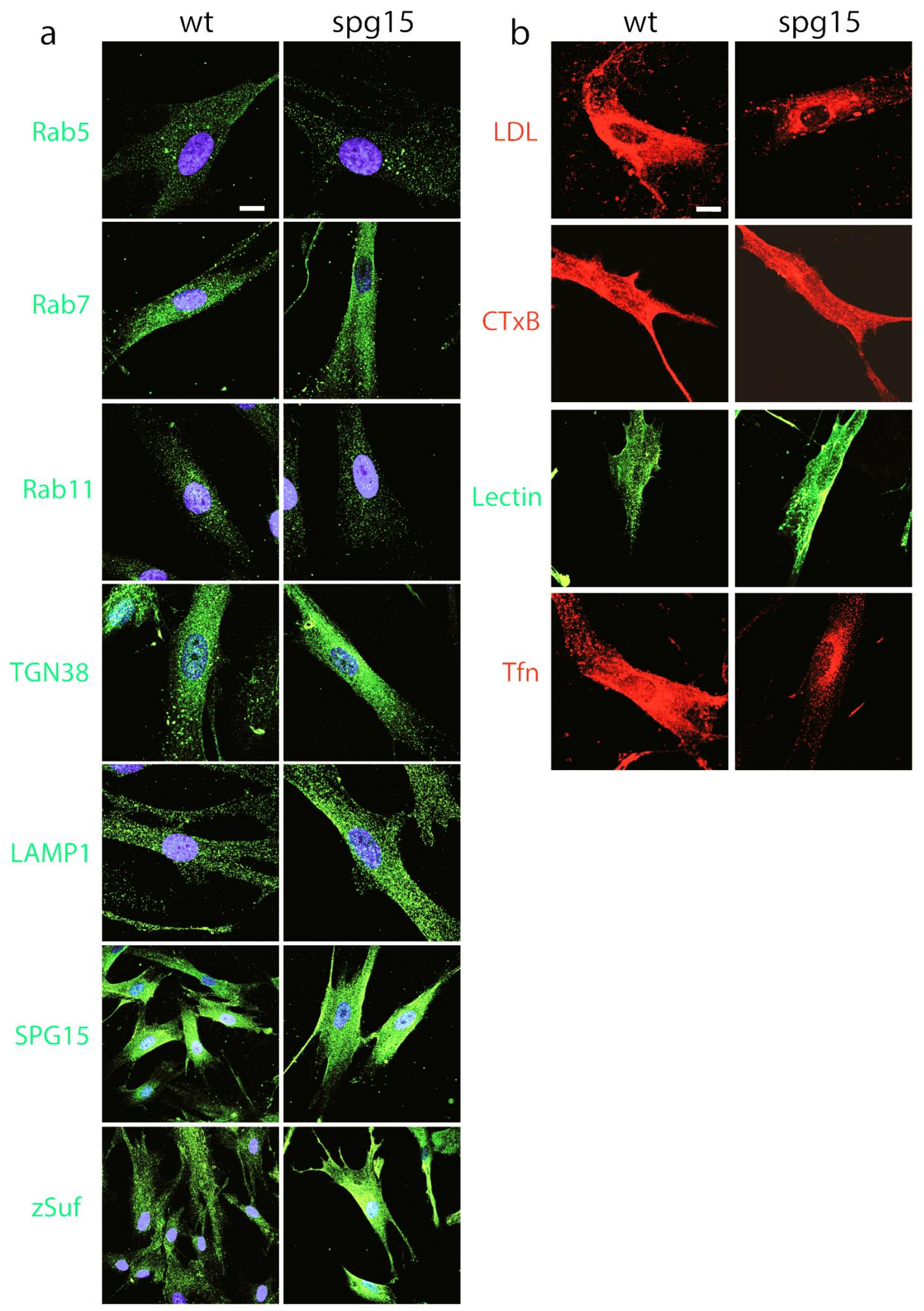




\subsection{Mitochondrial defect in suf mutant oocyte}

Mitochondria is one of the sensitive organelle of the cell and a defect in mitochondria causes neuron degeneration and cell death. Suf is also known to localize to the mitochondria. The Lysosome and mitochondria are connected for transport of lipids and the lysosome is necessary for removing defective mitochondria by mitophagy to keep the cell healthy. Therefore, I wanted to see if the mitochondria were normal in the suf mutant oocyte since the maternal mitochondria is stored in the oocyte (Nora Crascanta, IMPRS masters in molecular biology, Lab rotation project). Staining for mitochondria with different membrane potential based dyes revealed that the structure and organization is disrupted in the suf mutant, also we observed accumulated mitochondria via electron microscopy (Fig. 54 a \& b). This result suggests that suf might have role in mitochondrial function directly or indirectly through the lysosome.

\section{Figure 54. Suf mutant show disturbed mitochondrial network.}

a. Mitochondria are stained with TMRE. Mitochondria are in red. Left panel from wt oocyte and right panel from suf mutant oocytes. First two rows shows the overall distribution of the mitochondria around the nucleus in the wt and suf mutant shows disturbed distribution. Third and fourth rows shows the mitochondria in the Baliani body structure. The last row shows the cortex region of the oocyte. b. Electron micrograph showing mitochondrial accumulation in the suf mutant compared to the wt distribution. Scale $-50 \mu \mathrm{m}$ for panel a and $2 \mu \mathrm{m}$ for panel $\mathrm{b}$. 
a
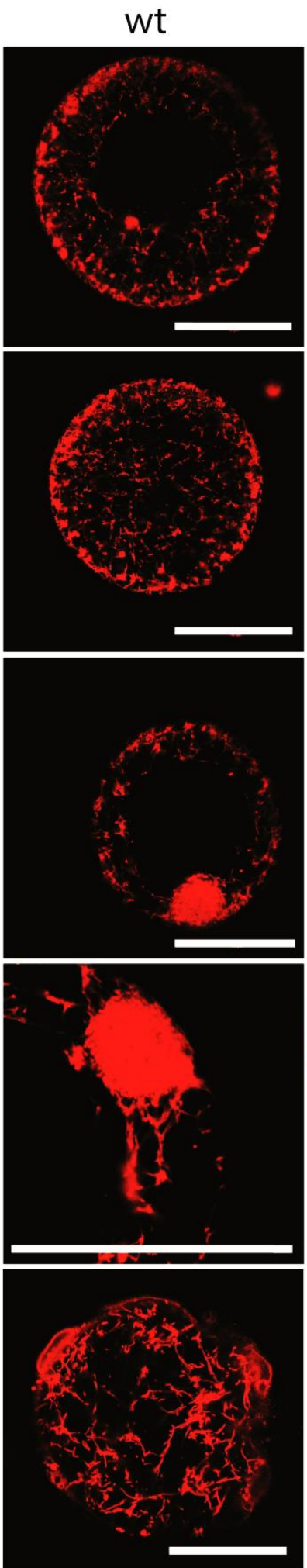

suf
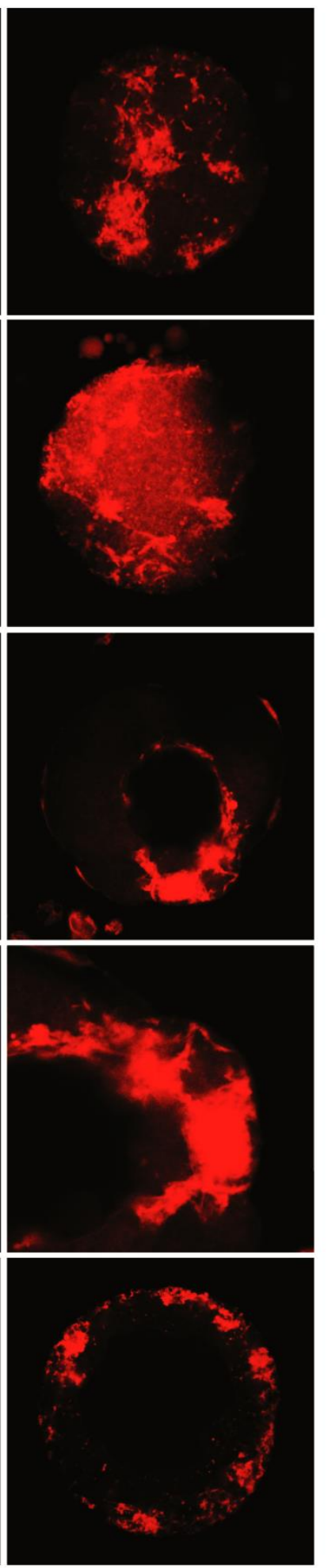

$\mathrm{b}$

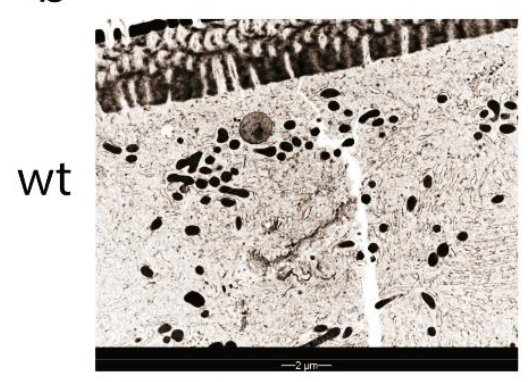

suf

Esto. OID

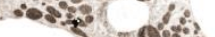

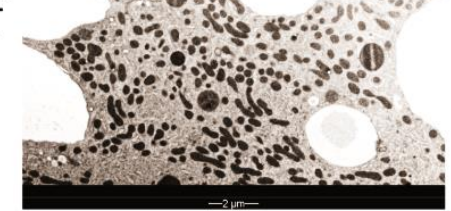

\subsection{Cloning other opaque egg mutants}

Suf is one of the opaque egg mutants, which show same phenotype, suggesting all have a defect in yolk degradation. The mutants might interact with suf or work in same pathway. So finding the gene mutated in other mutanst would provide as new candidate for 
HSP. To analyse if other mutants have lysosomal defect, lysosome were stained in sunny side up mutant ooctyes and electron microscopy was used to study the ruehrei mutant oocyte. Interestingly, both mutants show fragmented oocyte like suf mutant (Fig. 55). Parellely, ruehrei mutant has been positionally cloned as TOMM70a using RNA sequencing, suggesting a connection between mitochondria and lysosome biogenesisn and function. Recent interactome study predicted that TOMM70a will be a HSP causing gene in human consistant with our study. It will be interesting to know if these mutants also having defect in secretory pathway. So, these opaque egg mutants will be a good model to study the HSP disease genes and function. 

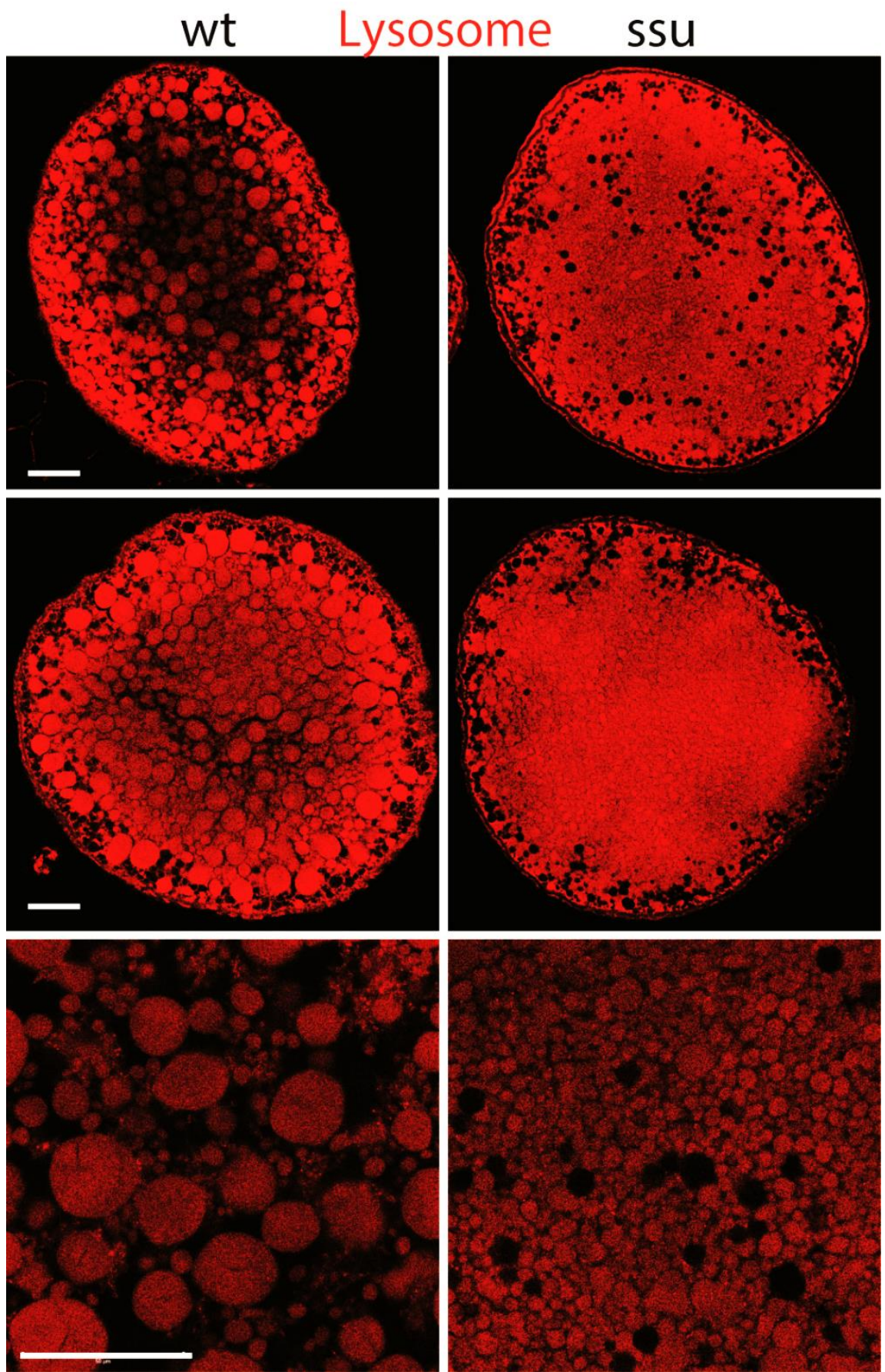

Figure 55. Other opaque egg mutants also show fragmented lysosome.

a. Picture shows electron micrograph from ruehrei mutant oocyte compared to wildtype. $b$. Staining for lysosome in red in sunny side up mutant oocytes compared to wild type oocyte. Scale $-50 \mu \mathrm{m}$. 


\section{Discussion}

Oogenesis is an essential part of any sexually reproducing organism's life to propagate the next generation. During oogenesis, the oocyte undergo many dynamic changes and maturation after growth from the follicle cells. Most importantly, they accumulate nutrients and essential factors from the mother, which are necessary during fertilization and early development. During this process, the oocyte actively use endocytosis and other transport pathways to attain the the required maturation. Many regulators have been identified in the yeast and other invertebrates but the genetic screen in C. elegans discovered a novel factors and cellular compartments specific to the multi cellular organism, suggesting that the vertebrates require many more essential factors compared to single cell organisms for oocyte maturation. So a genetic screen for maternal effect mutants in zebrafish was carried out (Dosch et al.,2004) and many mutants were discovered having a defect in oogenesis. Thus providing a valuable tool to study the functions of those genes in vertebrates.

Here, we take advantage of zebrafish genetics and its oocyte with their high vesicle trafficking activity to describe novel role for the SPASTIZIN homolog Souffle. The present study shows that during zebrafish oogenesis Suf/Spastizin is essential for the maturation of secretory granules by sorting lysosomal cargos from immature secretory granules. Suf/Spastizin colocalizes with Rab11b to an intermediate compartment during the formation of dense-core vesicles (DCV) which are competent to fuse with plasma membrane during exocytosis after fertilization. Our results show that suf plays crucial role in sorting of cargos and vesicle fission from immature secretory granule (ISG) during secretory granule maturation in zebrafish oocytes (Kanagaraj et al.,2014). Also, the current study shows the defect in lysosomal biogenesis and degradative function is a consequence of the sorting defect in ISG. Furthermore, this study explains the role of suf in sorting lysosomal cargos like cathepsin proteases from immature secretory granule to lysosome through VPS41 an AP3 mediated transport pathway. In conclusion, we discover the novel role of suf in sorting of lysosomal cargos from the secretory pathway which leads to form dense core vesicle which are competent to fuse with plasma membrane.

\subsection{Souffle regulates Rab11b positive secretory vesicle.}

A striking phenotype in suf oocytes is the accumulation of Rab11b positive vesicles. However, the recycling is not affected in suf mutants. Nevertheless, in fibroblasts it is 
unusual that transferrin does not colocalize with Rab11b and consistently, none of the other phenotypes besides the defect in cytokinesis supports a role for Suf/Spastizin in endosomal recycling. Interestingly, in certain human cell types, secretory cargoes also pass through Rab11-positive endosomes (reviewed in Taguchi.2013). Polarized tissue culture cells spatially and functionally separate Rab11a/Transferrin and Rab11b (Wang et al.,2000; Lapierre et al.,2003a) This remarkable separation of Rab11a and -b is mostly observed in polarized epithelial cells, which form the Rab11-positive sub apical compartment (SAC)/common endosome as a central sorting hub for recycling (reviewed in Hoekstra et al.,2004). Although the zebrafish oocyte is highly polarized along the animal-vegetal axis, polarity at the level of vesicle transport has not been previously described. Therefore, the Rab11b positive compartment in the zebrafish oocyte requires further analysis to confirm its homology to the sub apical compartment of human epithelial cells. Furthermore the Rab11 has been shown to regulate secretory granule transport and exocytosis during oogenesis in C.elegans (Sato et al.,2008) and they are regulated by cell cycle components during cell division (Bembenek et al.,2007).

Another study performed in rat neuroendocrine PC12 cell cultures implicated, Rab11b in the formation of dense-core vesicles (Khvotchev et al.,2003; Mikhail Khvotchev.2003). This report demonstrated that Rab11b, but not Rab11a and Rab25 of the Rab11 protein family, are involved in secretion. Moreover, Rab11b was suggested to control sorting of secretory cargo in neuroendocrine cells. This result in rat tissue culture also provides an insight into the molecular mechanism for Suf/Spastizin function, which is consistent with our results in the zebrafish oocyte. On repeating their experiments in unpolarized human fibroblasts (e.g. HeLa cells) showed different effects with Rab11b (Khvotchev et al.,2003). This might explain why the Spastizin homolog was not implicated in regulated secretion before our study, since it was mostly analyzed in unpolarized human fibroblasts (Sagona et al.,2010; Hirst et al.,2011; Hirst et al.,2013a). It remains to be determined to which degree our results from the zebrafish oocyte is comparable to mammalian tissue culture experiments, though we have shown that the oocyte behaves like polarized and neuroendocrine cells for active secretion.

\subsection{Souffle localizes to secretory granule and endosomal compartment.}

Our current study discovered that suf localizes to endosomal compartments and secretory granules. Recent studies in other systems showed multiple localization places in different cellular organelle, which includes endosomal compartment consistent with our 
studies. Immuno-staining and electron microscopy studies showed that suf localizes to neuronal tissues but not in astrocytes, spinalcord, motor cortex, cerebellum and also expressed ubiquitously in embryonic tissue. Spastizin localizes to both cytoplasmic and nucleus in cellular level. Further studies at a subcellular level showed that spastizin localizes to microtubules, trafficking vesicles, ER, mitochondrial membrane. Interestingly, they did not observe any co-localization with early endosomal marker EEA1, lysosomal marker LAMP2 and Golgi (Murmu et al.,2011), contrast to the strong vesicular localization shown in previous study (Hanein et al.,2008). Although the localization of spastizin in many organelles is evident including the present study, they all have few common localization signals in lysosome and vesicular structures. In addition, studies in patient fibroblast showed accumulation of enlarged lysosome but their degradative function was not impaired (Renvoise et al.,2014). These multiple results suggest that suf play important role in endolysosomal system consistent with their defect in lysosome.

Another study in cultured cells showed the role of suf in cytokinesis by their localization in the midbody as well as in centrosome (Sagona et al.,2010; Sagona et al.,2011). However, other studies did not find in any other cells except that our study showed defect in maternal cleavage after fertilization. Furthermore, they also showed that suf interacts with KIF13a to reach the midbody, where KIF13a has been earlier known to play role in secretion of Mannose 6-phophate receptor to plasma membrane (Nakagawa.2000a; Nakagawa.2000b)(Nakagawa et al., 2000) indicating their possible role in secretion. Although the current study has genetic evidence for the role of suf in secretory granule maturation using electron microscopy and defect in lysosomal biogenesis, but the connection remains to be explored.

\subsection{Suf regulates secretory granule maturation.}

Our functional and confocal imaging studies show that suf is necessary for secretory granule maturation. Furthermore, electron microscopy study showed that sorting and fission is necessary for the dense-core vesicle (DCV) formation during maturation. The data from human fibroblasts showed that Suf/Spastizin interacts with the novel AP5 complex and depletion causes defect in formation of multi vesicular-bodies (MVB) and generates empty MVBs (Hirst et al.,2011; Hirst et al.,2013a). Consistently, the mouse knock out demonstrates genetically that Spastizin is critical in vivo during formation of lysosomes (Khundadze et al.,2013). In zebrafish, we also detected fragmented lysosomes in Suf/Spastizin mutant oocytes in addition to the defect in secretion. Moreover, mutations in 
AP5 and SPASTIZIN cause HSP in humans confirming genetically that both proteins are involved in the same process. However, Suf/Spastizin also binds to Kif13a, which was implicated by tissue culture overexpression experiments to regulate secretion (Nakagawa et al., 2000). Since all these studies were carried out in different organisms and different tissues, it is difficult to extract the precise function of Suf/Spastizin.

A major question arising from our work is that how the various observed phenotypes are could be reconciled. Two alternative scenarios integrate all results: Suf/Spastizin primarily acts in MVB/lysosomes and the maturation defect of secretory vesicles is secondary e.g. through retrograde transport to the Trans-Golgi-Network (reviewed in Bonifacino and Rojas.2006). Alternatively, Suf/Spastizin primarily acts in immature secretory granules and the lysosomal defect is secondary e.g. through sorting of the mannose-6-phosphate receptors transporting hydrolytic enzymes in clathrin coated vesicle to the lysosomes. (Fig.56) (Morvan and Tooze.2008). Currently, we favor the second model, since defects in retrograde transport were never reported to affect the formation of the dense core in secretory granules as in the suf mutant. This model would also predict that cargo sorted away in immature secretory granules is necessary for homotypic lysosome fusion as described in other cells (Pillay et al.,2002). Failure in forming dense core region confirms the role of suf in secretory granule maturation. Two main models have been proposed to explain how the appropriate cargos are sorted into newly generated DCVs: sorting by entry and sorting by retention. The sorting by entry model assumes the existence of sorting signals and receptors that would actively sort cargo into forming DCVs (Arvan \& Castle, 1998; Borgonovo et al, 2006; Kim et al, 2006; Tooze et al, 2001). Such short signal motifs have been identified in DCV cargos such as provasopressin, pro-oxytocin, pro-opiomelanocortin, $\mathrm{CgA}$, and $\mathrm{CgB}$, which are sufficient for DCV targeting (Cool et al, 1995; Glombik \& Gerdes, 2000; Huttner et al, 1991; Tooze, 1998). In contrast, the sorting by retention model suggests that DCV cargo could passively enter maturing DCVs and then be retained during DCV maturation either by active retention in lipid domains or by its aggregation within iDCVs under low $\mathrm{pH}$ and high $\mathrm{Ca}^{2+}$ concentration (Glombik \& Gerdes, 2000; Hosaka \& Watanabe, 2010 (Hannemann et al.,2012)). Furthermore, it has been shown in the case of secretogranin III (SgIII) that there are direct interactions between aggregated DCV cargos and cholesterol-rich membrane domains of DCVs (Hosaka \& Watanabe, 2010). Interestingly, study in C.elegans also showed Rab2 involvement in retaining DCV cargo proneuropeptide NLP-21 in the aggregation, depletion of Rab2 leads to mis-targeting of those cargos to lysosome 
(Hannemann et al.,2012). Also the current study found the defect in CCV fission as shown in the picture the fission is a necessary from immature secretory granule to remove the nonaggregated cargos which are destined to somewhere else than the plasma membrane. Previous studies have shown that removal of VAMP4 and Syntaxin 6 is necessary for dense core vesicle maturation and mutation in AP1 binding motif of VAMP4 leads accumulation of VAMP4 in mature granules (Eaton et al.,2000). This clathrin-dependent membrane remodeling process functions as a proof reading mechanism to ensure proper DCV content and correct membrane composition of mDCVs (Morvan and Tooze.2008). This is necessary, since it has been shown that only mDCVs are able to undergo efficient, stimulus-dependent exocytosis (Eaton et al.,2000). The final steps of DCV maturation, prior to fusion, are condensation of cargo molecules by further acidification and removal of water (Kim et al.,2006). Furthermore, study has shown that retrograde transport also essential to make functional DCV (Hannemann.2012; Hannemann et al.,2012). Another study in Tetrahymena showed that the lysosomal receptor is necessary for sorting the lysosomal cargo during the secretory granule maturation (Briguglio et al.,2013). Although interaction with AP5 with current study showing the failure in dense core formation confirms that suf is essential in sorting and maturation, but it is necessary to find the cargo and receptor which are sorted by suf in case if they play role in sorting lysosomal cargo. These open questions makes it clear that additional studies are necessary to determine the precise role of Suf/Spastizin and whether a defect in lysosome formation or secretion or even another process leads to neuronal degeneration in HSP patients. 


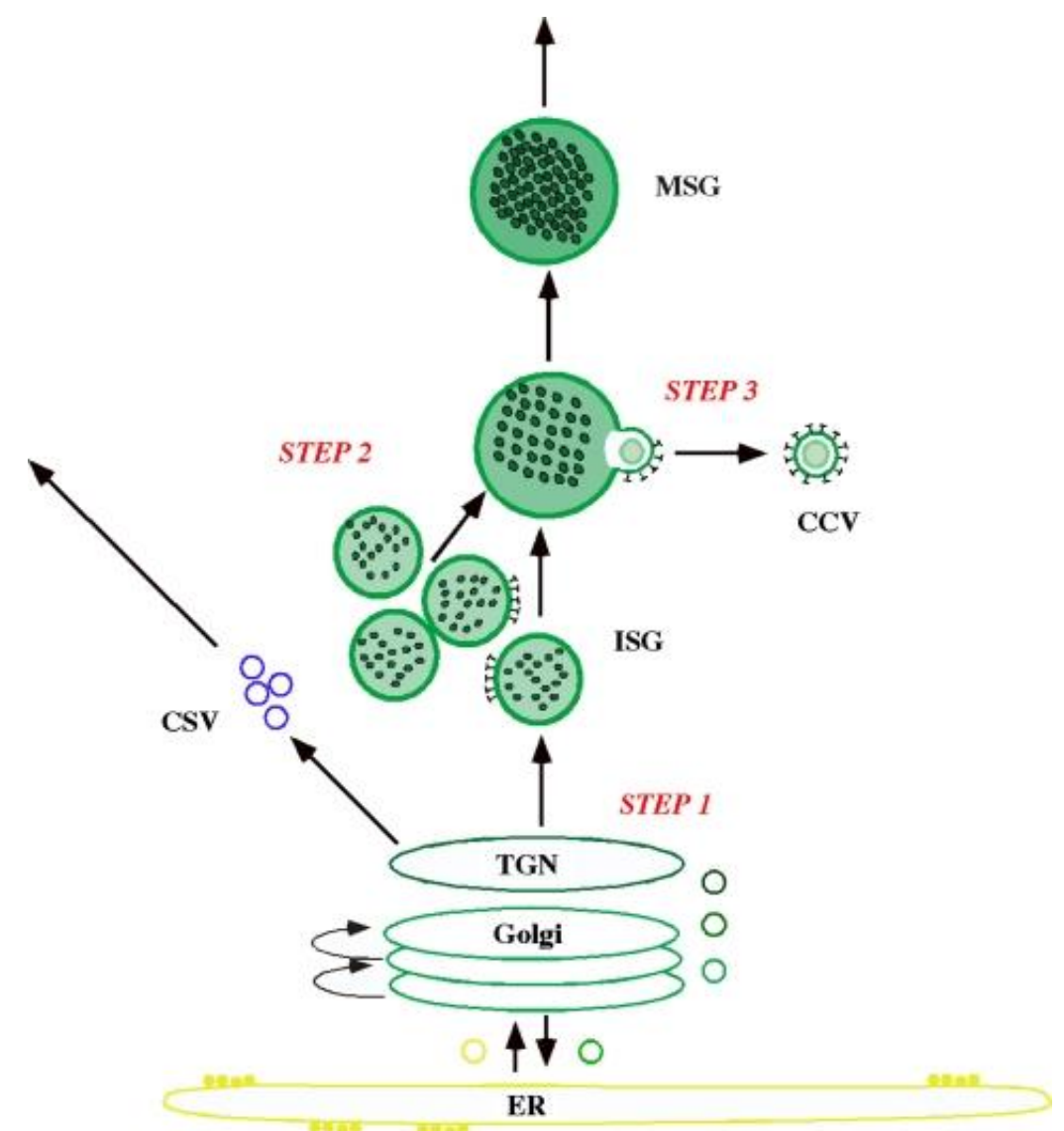

\section{Figure 56. Key steps in secretory granule biogenesis.}

Step 1 depicts the formation of ISGs from the TGN, Step 2 the homotypic fusion event between ISGs, and Step 3 the clathrin-mediated membrane remodeling event which leads to formation of matured secretory granule(Morvan and Tooze.2008).

\subsection{Suf is essential for early mitotic cytokinesis}

Notably, our data implicates Spastizin/Suf for the first time in the maturation of secretory granules. Comprehensive studies already have described different roles for Spastizin/Suf, e.g. as FYVE-CENT in cytokinesis (Sagona et al.,2010; Sagona et al.,2011) and as Spastizin binding to AP5 in the formation of multi vesicular-bodies (Hirst et al.,2011; Hirst et al.,2013a). We could genetically confirm a previously described role for Spastizin/Suf in cytokinesis during the maternally controlled cell divisions, but the question remains how these different functions can be reconciled. However, we don't find any cytokinesis defect in the adult homozygous suf mutant fish and the suf mutant oocytes also succeed in meiotic division indicating the need of suf in cytokinesis only during early maternal period of development in zebrafish or the function is taken over by other proteins in the adult although the mutant allele is present in all the tissues during development. Furthermore, morpholino mediated knockdown of suf also showed no defect in cytokinesis 
consistent with the previous results that this specific mutation affects early mitotic division. Remarkably, a study in Drosophila showed that dynamin is necessary for budding of vesicle from rab11 positive compartment during cllularizaion, a process similar to maternal cleavage and genetic mutant for dynamin called shibire shows same cellular phenotype in electron microscopy like soufflé mutant accumulating clathrin buds (Pelissier et al.,2003). Excitingly, secretory granules are also required during cytokinesis in tissue culture cells (Bukoreshtliev et al.,2012) and plants for adding new membrane during cell plate formation (Reichardt et al.,2007). The possible role of secretory vesicles during cytokinesis would be to deliver new membrane to the daughter cell and recruiting the necessary components to complete the abscission. Although the recycling endosome has been suggested to play this transport based on presence of Rab11 and Rab35 on the vesicle but would be necessary to identify their origin to differentiate from secretory vesicle involvement (Kouranti et al.,2006; Polevoy et al.,2009; Bembenek et al.,2010; Neto et al.,2013; Sechi et al.,2014). Current study also showed defect in Dynamin which also shown to be necessary for cytokinesis (Konopka et al.,2006; Gonzalez-Jamett et al.,2013). The current study finds that suf is necessary for maternal cleavage consistent with other studies in other organisms. Therefore, it would be interesting to investigate the zygotic role of Spastizin/Suf on secretory vesicles during cytokinesis in adult.

\subsection{Souffle is necessary for lysosomal biogenesis and function.}

The current study discovered that suf mutant accumulates fragmented lysosomes and failed to degrade the yolk protein, which creates the opaqueness. The fragmented lysosome is a classical phenotype of class C VPS mutants from yeast screen indicating a connection to HOPS complex role in lysosomal fusion. Is suf mutant failing to recruit HOPS on lysosome? Or are they not delivered to lysosomes? Or something else is missing to complete the fusion. Also other studies in ZFYVE26 mutant mice showed a similar defect in the lysosome and also failed to degrade lipid accumulation which are LAMP1 positive confirming that the suf is necessary for lysosomal function in two different genetic mutants (Khundadze et al.,2013). In addition, they also notice a reduction in neuronal cells in cerebellum region in adult and defect in hind leg balance relating their symptom to Spastizin mutant HSP patients. In human as well as in this mouse model the symptom only appears at very late age while some studies in fibroblast showed that the spg 15 mutant has a defect in lysosomal function contrast to our study as well as the human symptom (Renvoise et al.,2014). Further studies using cell culture suggested suf role also in 


\section{Discussion}

autophagy supporting previous study of Beclin1 and spastizin interaction during autophagosome maturation in cell culture. Moreover, they also showed that Beclin1UVRAG-Rubicon complex forms in the absence of spastizin and the mutant spastizin loose the connection to this complex resulting in accumulation immature autophagosome (Chiara Vantaggiato.2013; Vantaggiato et al.,2013). It would be interesting to know if they fail to fuse with lysosome, which will connect the defect in lysosome and accumulation of autophagosome; a possible consequence of fusion defect than spastizin direct role in autophaosome formation.

HOPS complex plays crucial role in all lysosomal fusion with other compartments. Previously VPS41, a HOPS subunit showed similar defect of fragmented lysosome as well as defect in sorting multiple lysosomal enzymes (DEREK C. RADISKY.1997). Meanwhile, AP3 an adaptor protein has been shown to transport lysosomal cargos from Golgi to vacuole and requires VPS41 to fuse with vacuole. This pathway transports tSNARE VAMP3 to lysosome, which is necessary for the fusion. So loss of this transport will affect the homotypic lysosomal fusion and the cargo vesicle fusion with lysosome causing fragmented lysosome and defect in lysosomal transport (Cowles.1997). Another study also showed that AP3 transports VAMP7, which is necessary for lysosomal fusion (Kent et al.,2012). AP3 and VPS41 exhibits similar lysosomal phenotype and in addition fails to form a dense core vesicle, leads us to think that suf might play together in this pathway or similar pathway.

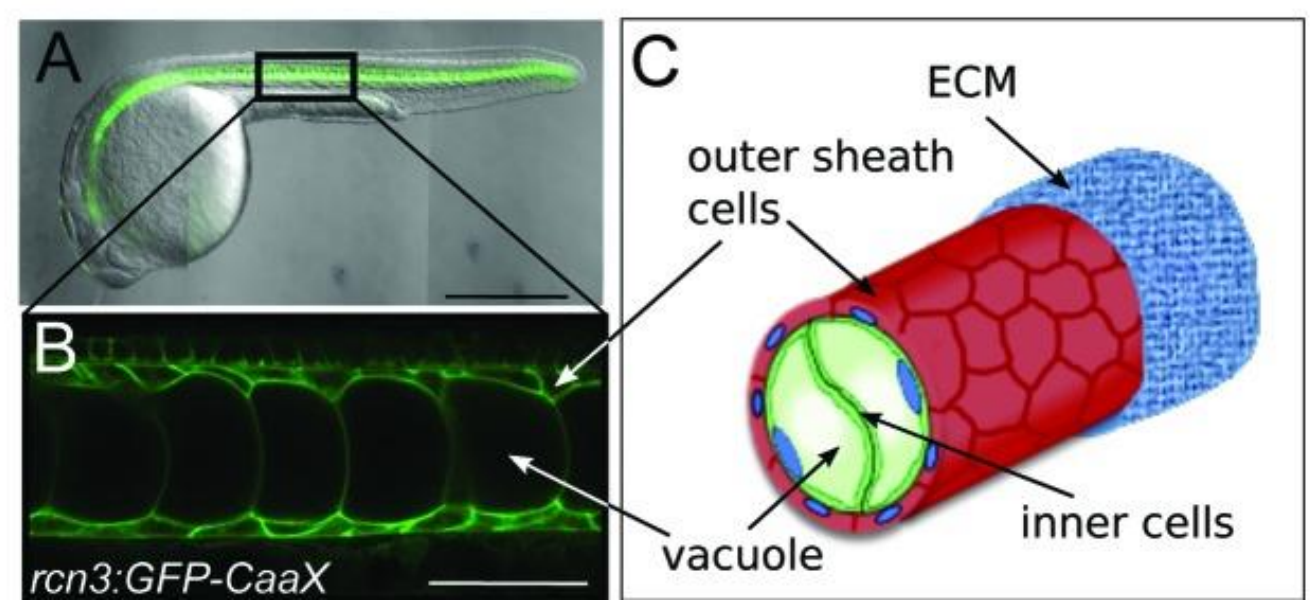

Figure 57. Notochord vacuoles in zebrafish. 
(A) $24 \mathrm{~h}$ post fertilization (hpf) zebrafish embryo expressing membrane GFP in the notochord. Anterior is left, posterior is right, dorsal is top, and ventral is down. (B) Higher magnification of notochord. (C) Cartoon shows notochord structure of inner vacuolated cells surrounded by an epithelial-like sheath of cells (Kathryn Ellis.2013).

Surprisingly, suf morphant embryos show defect in the lysosome during notochord development. It is known that the proper lysosomal organization within notochord is essential for spinalcord development (Fig. 57) and defect in lysosome arrangements or morphology creates twisted tail phenotype in zebrafish (Ellis et al.,2013). The picture shows proper large lysosome arrangement in the notocord of zebrafish in green (Fig. 57 Panel B). Suf knockdown in embryo shows same tail twisted phenotype and misshapen lysosome in the notochord further confirming role of suf in lysosomal biogenesis. Furthermore, LIMP2 mutant zebrafish shows fragmented lysosome in notochord and the whole body bends upwards due to failure in spinal cord development similar to suf knockdown embryo. LIMP2 is a lysosomal membrane protein and involves in transport of beta glucocerebrosidase enzymes from secretory pathway to lysosome independent of Mannose 6-phophate receptor supporting the role of suf in transporting cargos from secretory pathway (Abigail Diaz-Tellez et al.,2012). Also lysosomal receptor VPS10/ Sortilin transport is necessary for DCV formation in Tetrahymena (Briguglio et al.,2013). Interestingly, mutants of VPS41, AP3 and LIMP2 show cellular defect in lysosome and DCV formation similar to suf mutant indicating that suf involves in lysosomal cargo transport and the lysosomal defect might be consequence of sorting defect in secretory pathway. As suf mutant show fragmented lysosomes, it is likely that AP3, VPS41 and Suf involves in transport of similar cargo to the lysosome.

\subsection{Suf transport cathepsin proteases enzyme to lysosome.}

Suf mutants fail to degrade the yolk although the yolk reaches the fragmented lysosome suggesting that the lysosome is missing something or the fusion is necessary to activate the function. Acidification is another essential event in lysosome for the enzymatic activity, although suf mutants show positive for lysotracker eliminating acidification issues but still the lysosotracker sometimes does not show minor changes below $\mathrm{pH}$ 5.5. The main enzymes involved in yolk degradations are cathepsin D, B, F and L (Lubzens et al.,2010) and vacuolar ATPase function is essential for activation of these enzymes in the lysosome by reducing the $\mathrm{pH}$. Further studies on inhibition of vATPase showed defect in yolk degradation and oocyte maturation (Selman.2001). Cathepsin L has been shown to play 
role in yolk processing in C.elegans (Murray.2004). Also these cathepsin enzymes are transported differently and function in different time during oogenesis (Lubzens et al.,2010). Current study shows that cathepsin B, F and L is not delivered to the lysosome while cathepsin D is in lysosome in suf mutant. These enzymes are transported from immature secretory granule using multiple receptors in different way depending on the cell or animal model. Cathepsin D has been shown to transport mostly by M6PR and sortilin consistent with our results where both receptors reach lysosome and cathepsin D also in the lyososme (Judith Klumperman.1998). But Cathepsin B, F and L accumulates in secretory granule and some of them are secreted out indicated by the strong staining on outside membrane and follicle cells. Interestingly M6PR knockout mice shows normal distribution of these enzymes in the lysosome suggesting alternative pathway by sortilin.

The oligosaccharide chains of the lysosomal hydrolases acquire terminal mannose6-phosphate (M6P) residues as they pass through the Golgi complex. These M6P tags bind to MPRs, which contain cytosolic sorting motifs for packaging into transport vesicles. Specifically, M6PRs contain 'acidic cluster dileucine (AC-LL) signals' that are recognized by GGA (Golgi-localizing, $\gamma$-adaptin ear domain homology, ARF-binding protein) adaptor proteins. Upon binding the AC-LL signal, GGA adapter proteins recruit clathrin from the cytoplasm and a clathrin coat is assembled at the TGN. After coat formation, the vesicles pinch off from the TGN and fuse with endosomal intermediates where the low internal $\mathrm{pH}$ $(\mathrm{pH}$ 5.5) triggers dissociation of lysosomal enzymes from M6PRs.(Kienzle and von Blume.2014). Inhibition of vATPase causes defect in lysosomal cargo segregation with secretory granule cargo creating mixed organelle and also inhibited regulated secretion (Sobota JA.2009). Although acidification is required in both endocytosis and the secretory pathway, they are regulated differently to differentiate the level of acidification by regulating number of vATPase on the membrane (Dettmer et al.,2006). Although, a block in cathepsin and vATPase subunit'd' transport is seen in suf mutants, M6PR and sortilin are transported properly to the lysosome suggesting that suf might function in a different pathway. Further, it will be necessary to know if LIMP2 is sorted properly in suf mutant since the notochord phenotype looks similar to limp2 mutant and limp2 is another receptor mediating lysosomal transport although only involved in beta- Glucocerebrosidase transport independent of M6PR (Reczek et al.,2007; Zeigler et al.,2014). But, a recent study showed that LIMP2 is transported with M6PR pathway (Zhao et al.,2014). Also the function of GGA1 and AP1 is intact in suf mutant since cathepsin D reaches lysosome and 
initial processing of vitellogenin yolk precursor into yolk protein happens in suf mutant, further supporting that M6PR and Sortilin mediated pathway is intact in suf mutant.

In yeast, apart from sortilin mediated transport, lysosomal cargos are transported in ALP pathway, which is mediated by VPS41 and AP3. Furthermore, HSP in human is caused by mutation in VPS41 or AP3 or Suf or AP5 rather than mutation in M6PR or VPS10, indicating that these proteins are required in specific conditions or for sorting specific cargos compared to M6PR and sortilin. In secretory granules they fail to form dense cores and in the lysosome some of the cargos are missing like cathepsin and vATPase subunit' $d$ '. Furthermore, the secretory granule marker transported to other compartments in suf mutant including the lysosome suggesting that acidification is also affected in this line. Since DCV formation requires acidification to aggregate the cargos, which leaves the lysosomal cargo available for sorting to lysosome. If the dense core did not form then the lysosomal cargo will mix with secretory granule cargo and fail to transport to lysosome creating mixed organelle as reported earlier. Maintaining acidic $\mathrm{pH}$ is critical for the proper association of granulogenic proteins with lipid rafts and formation of DCVs. Taupenot et al. (Taupenot et al.,2005)reported that treatment of PC12 cells with the V-ATPase blocker, Bafilomycin A1, resulted in the significant reduction in sorting of chromogranin A to DCVs. Furthermore, electron microscopic analyses of these Bafilomycin A1-treated PC12 cells, as well as AtT-20 cells, revealed reduced numbers of secretory granules with dense cores (Tanaka et al.,1997), suggesting that the acidification mediated by V-ATPase is crucial for the formation of DCVs. Initial processing of prohormones, for example, proinsulin, POMC, and secretogranin II, begins in the ISGs. Continued acidification in the ISGs is necessary to activate the PCs and processing of the prohormones and proneuropeptides. Indeed, when the $\mathrm{pH}$ gradient was abolished by a VATPase blocker in AtT-20 cells, processing of POMC was severely inhibited, and it was secreted through the constitutive secretory pathway. If the condensation fails the regulated cargo will take constitutive path and get secreted out. These studies support that the defect in lysosome is a consequence of sorting and budding of cargos from immature secretory granule by lysosomal transport pathway independent of M6PR and Sortilin.

The yolk degradation in oocytes has been studied extensively, still the activation of these cathepsin proteases are unclear. Yolk degradation is necessary but only defect in yolk degradation does not affect the development as noticed in other mutant $d p 14 n b$ (Dosch et al.,2004) where the egg cytoplasm is opaque but they further develop, suggesting that other 
opaque egg mutants having multiple defects or the yolk degradation is a consequence consistent with suf function in secretory granule maturation. Cathepsin D involves in primary yolk cleavage and other cathepsin B, F and L involves in secondary processing consistent with the presence of different yolk protein in suf mutant which are processed from vitellogenin by cathepsin D which is inhibited when function of vATPase is blocked (Raldua.2006). Previous studies by Carnevali et al. (1999a) (carnevali.1999) on the sea bream (Sparus aurata) have found high levels of cathepsin D and B activities in isolated early vitellogenic oocytes, whereas cathepsin L activity is higher than that of cathepsin B and D in follicles at mid-vitellogenesis stage and the authors suggested that cathepsin $\mathrm{L}$ is necessary during oocyte maturation. In benthophil teleosts, however, the enzymatic machinery involved in yolk protein processing during oocyte maturation appears to be different. In the killifish, the enzyme activity of cathepsin L shows a dramatic decrease during oocyte maturation both in vivo and in vitro (LaFleur et al., 2005). By contrast, the activity of cathepsin B increases transiently during maturation, coincident with the time of maximum degradation of the $122 \mathrm{kDa}$ LvH1 (LaFleur et al.,2005). These observations suggest that cathepsin $\mathrm{B}$, rather than cathepsin $\mathrm{L}$, is the major protease involved in LvH1 degradation in killifish oocytes. Interestingly, a similar pattern of cathepsin B and L enzyme activities has been reported during late maturation of zebrafish oocytes in vivo (Carnevali et al.,2006). During this process, Vgs are cleaved into smaller molecular weight polypeptides, known as the yolk proteins, lipoproteins (lipovitellins (Lvs)), highly phosphorylated proteins (phosvitin and phosvetes) and $\beta^{\prime}$ component that will be stored in the oocyte throughout the growth period. During oocyte maturation in marine teleosts, a second proteolytic event on yolk proteins is activated by the MIS before ovulation, which signifies the source of FAAs for oocyte hydration. During maturation the ordered crystalline structures within the globules generally disassemble, which confers on such oocytes their characteristic transparency in zebrafish (Fig. 59). The cartoon picture shows the different yolk protein generated by processing vitellogenin at different time points and when they are degraded during oocyte maturation (Fig.58). The time of acidification and yolk processing coincides in xenopus oocyte (Maxfield.1994). Since, cathepsin D is transported to the lysosome in suf mutants, it will be interesting to know, how cathepsin D is differentiated from other cathepsins by suf for sorting. 
Discussion

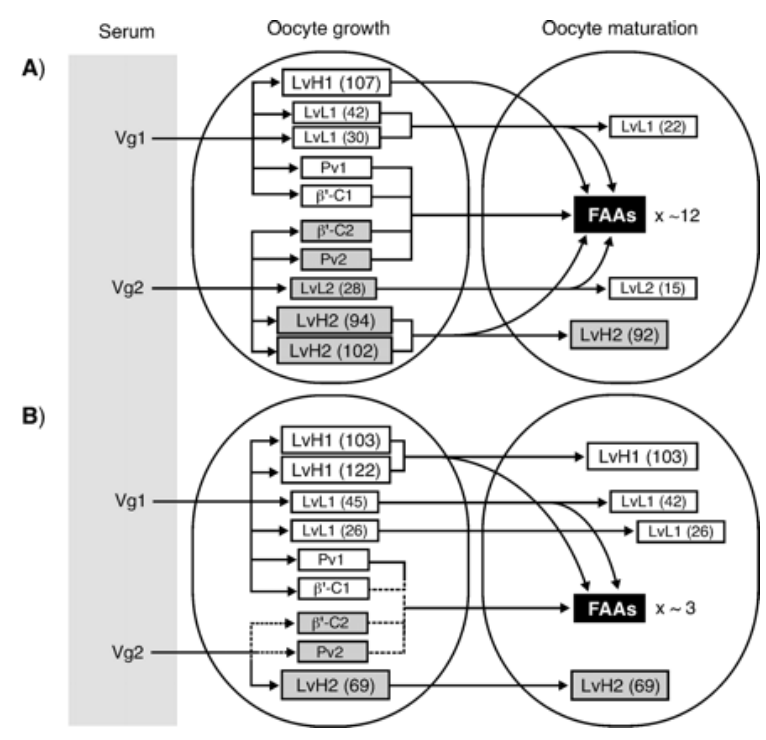

Figure 58. Yolk processing during oogenesis.

Schematic drawing of the flowchart describing molecular alterations of $\mathrm{Vg} 1, \mathrm{Vg}$, and $\mathrm{Vg}$ derived YPs during oocyte growth (vitellogenesis) and maturation in pelagophil (A) and benthophil (B) teleosts. Lipovitellin heavy chains $(\mathrm{LvH})$, lipovitellin light chains ( $\mathrm{LvL})$, phosvitins $(\mathrm{Pv})$, and $\beta^{\prime}$ components $\left(\beta^{\prime}-\mathrm{C}\right)$ derived from each $\mathrm{Vg}$ are indicated. Dotted lines indicate hypothetical pathways (LaFleur et al.,2005).
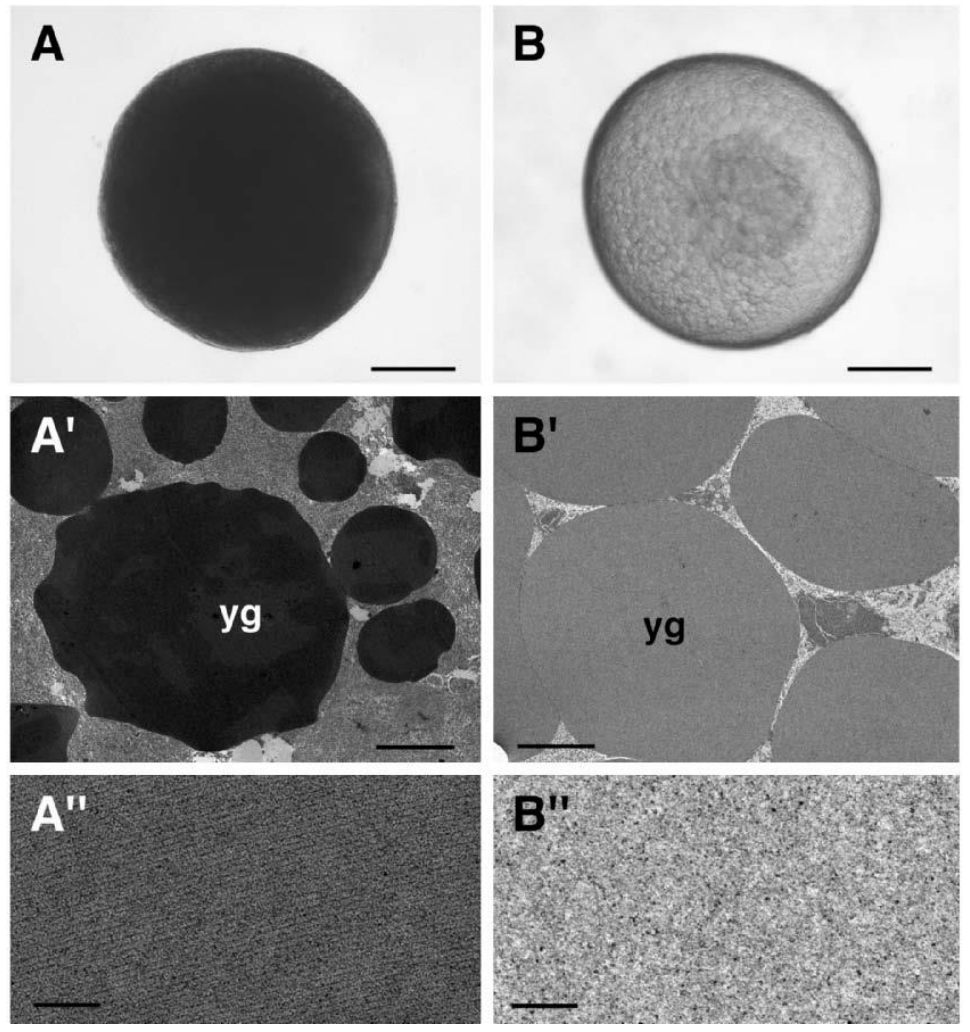


\section{Figure 59. Morphological and ultra-structural changes of yolk inclusions during oocyte maturation in Danio rerio.}

(A-B) Picture of immature (A) and mature (B) oocytes (Bars, $200 \mu \mathrm{m})$. (A'-B') Low magnification electron micrographs of yolk globules $(\mathrm{yg})$ in immature $\left(\mathrm{A}^{\prime}\right)$ and mature $\left(\mathrm{B}^{\prime}\right)$ oocytes. The crystalline nature of the yolk bodies is not apparent at this magnification (Bars, $5 \mu \mathrm{m})$. (A"-B') Higher magnification micrographs of crystalline $\left(\mathrm{A}^{\prime \prime}\right)$ or homogeneous $\left(\mathrm{B}^{\prime \prime}\right)$ yolk in immature and mature oocytes, respectively (Bars, $\left.0.2 \mu \mathrm{m}\right)$.

\subsection{Acidification is required for DCV formation and Yolk degradation}

The role of acidification in endocytosis and the secretory pathway is essential and regulated differently to maintain their $\mathrm{pH}$. The $\mathrm{pH}$ level plays crucial role in many protein interactions, ligand-cargo separation in endosome and formation of dense core aggregation in secretory granule maturation. Vacuolar ATPases is a multi-subunit proton pump acidifying various intra cellular organelles. Depletion of an individual sub unit shows different effect on different cellular functions. Inhibiting V-ATPase by Bafilomycin A1 leads sorting of POMC and secretogranin into constitutive secretory pathway in pituitary cells of xenopus, also reduces the number of secretory granule with dense core region (Schoonderwoert.2000). This reports suggests that the protein aggregation require low $\mathrm{pH}$ in immature granule and further low in mature granule to keep them together, if the $\mathrm{pH}$ is high then they don't form dense core similar to what is observed in suf mutant oocytes. The aggregation of proteins can happen by self-interaction or with other cargos and lipid raft.

A variety of proteins destined to be sorted into secretory storage granules form large complexes and aggregates within the lumen of the TGN; the low $\mathrm{pH}(\sim 6.4)$ of this compartment favors the formation of these clusters, which segregates them from other soluble proteins in the TGN. So-called immature secretory granules (ISG) bud from the TGN and transform into mature secretory granules (MSG) that contain a proteinaceous dense core. Mis-sorted non-granule proteins are progressively withdrawn by clathrincoated vesicles, eventually leaving the correct cargo protein in the MSGs. The ability of regulated secretory cargo, such as granins, to form aggregates is often an intrinsic feature of their protein structures. For example, they contain numerous acidic amino acids that drive clustering in the presence of millimolar $\mathrm{Ca}^{2+}$ and the slightly acidic $\mathrm{pH}$ of the TGN. These granin complexes interact directly or indirectly with cholesterol- and sphingolipidrich luminal membrane domains in the TGN. This interaction provides a driving force to 
induce budding from the TGN membranes to form ISGs. (Kienzle and von Blume.2014). Also some cargos bind to its receptor and bind to lipids for aggregation, CPE act as a receptor for POMC and pro-BDNF(Kienzle and von Blume.2014). Furthermore, continuous acidification of DCVs activate the enzymes (PCs, CPE), which are required for processing of proneuropeptides. The $\mathrm{pH}$ decrease from the TGN ( $\mathrm{pH} \sim 6.5-6.2)$ via iDCVs $(\mathrm{pH} \sim 6.3-5.7)$ to $\mathrm{mDCVs}(\mathrm{pH} \sim 5.5-5.0)$ is achieved by increases in the density of VATPase pumps and by decreases in $\mathrm{H}^{+}$permeability through the membranes $(\mathrm{Wu}$ et al, 2001a) (Hutton.1982). It has been shown that an acidic pH is not only crucial to activate processing enzymes, but also required for the formation of DCVs (Fig.60) (Tanaka et al.,1997; Wu et al.,2001; Taupenot et al.,2005).

\section{Figure 60. Aggregation of protein at low pH in DCV.}

After the secretory granule is established as an independent compartment, mature hormones and granin fragments move from the periphery to the inside of the secretory granules where they are further concentrated to form a dense core aggregate. Within the mature aggregate, neuropeptides/hormones are ready to be released upon a membrane fusion event (Watanabe.2010).

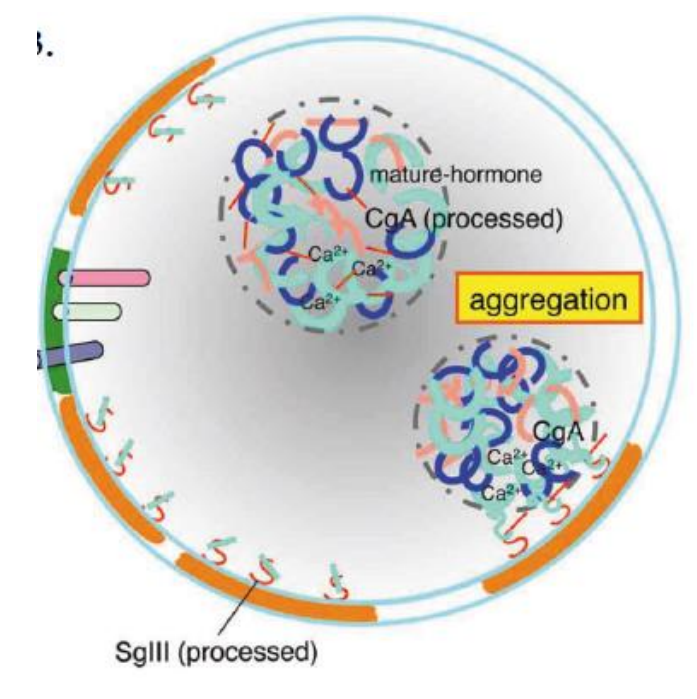

Taupenot et al., (2005) reported that treatment of PC12 cells with the V-ATPase blocker, Bafilomycin A1, resulted in the significant reduction in sorting of chromogranin A to DCVs. Furthermore, electron microscopy analysis of these Bafilomycin A1-treated PC12 cells, as well as AtT-20 cells, revealed reduced numbers of secretory granules with dense cores consistent with our electron microscopy studies, suggesting that the acidification mediated by V-ATPase is crucial for the formation of DCVs. So, we propose 


\section{Discussion}

based on our study that suf regulates sorting in ISG by removing lysosomal cargos resulting in DCV formation.

Yolk proteins are stored within yolk globules under mildly acidic conditions maintained by a V-ATPase (Sathish mallya.1992). In the embryo of most animals, a second V-ATPase mediated acidification of yolk inclusions triggers further digestion of the stored proteins. These are initially neutral, but they become acidic during development, causing maturation and/or activation of the pro-enzymes, pro-cathepsin $\mathrm{L}$ or pro-cathepsin $\mathrm{B}$, and yolk degradation. The activation of oocyte maturation in vitro by the MIS potentiates the activation of pro-cathepsin B into active enzyme and accelerates the hydrolysis of the 122 kDa LvH1(Fabra et al.,2006). Bafilomycin A1-induced increase of internal pH during oocyte maturation in vitro inhibits cathepsin B activation and enzyme activity, preventing yolk proteolysis, thus confirming that this protease is most likely the enzyme regulated by V-ATPase responsible for LvH1 degradation in killifish. However, it is unknown whether cathepsin B is located in the ooplasm or within yolk structures, as cathepsin D, where it might be activated through maturation of the pro-enzyme by the action of another cysteine proteinase or simply by changes in $\mathrm{pH}$ (auto processing). Cathepsins are normally delivered to lysosomes as proenzymes and since Bafilomycin A1 has been found to suppress indirectly the fusion of lysosomes into target vacuoles (Pillay et al.,2002), an inhibition of the delivery of cathepsin B to yolk globules during MIS-stimulated oocyte maturation is another potential mechanism (Babin.2007b; Babin.2007a). Hence, the details of cathepsin $\mathrm{B}$ and $\mathrm{L}$ regulation in fish oocytes, including the mechanism by which the V-ATPase becomes activated by hormonal stimulation, yet to be explained.

\subsection{Souffle regulate VPS41 mediated LAMP carrier pathway}

The commonly used lysosomal transport by M6PR and Sortilin are normal in suf mutant, but the lysosome misses cathepsin for yolk degradation and SNARE, which is necessary for their fusion. VPS41 and AP3 mediated LAMP carrier pathway also transport cargos to the lysosome directly and a defect in this pathway makes fragmented lysosomes and dense cores missing in the secretory granules (Pols et al.,2013) (Takeda et al.,2008) and defect in sorting of many lysosomal hydrolases (Radisky.1997). The AP3 and VPS41 mutants show similar phenotype like suf mutants. AP3 is known to function with VPS41 as an adaptor for lysosomal transport. Apart from its role VPS41 in lysosomal fusion event with HOPS complex it is also necessary for sorting cargo with AP3 which is required for DCV formation. Biochemical study showed that VPS41 binds to $\delta$-adaptin sub unit of AP3 
in transporting lysosomal cargo (Cowles.1997). Furthermore, when they used mutants for Vam3, the AP3 positive cargo accumulate suggesting that they need this SNARE on AP3 vesicle for the fusion with lysosome. But, when they used VPS41 and AP3 mutants they fail to form these intermediate vesicles indicating they are involved in sorting and budding of these vesicles (Fig. 61) (Peter rehling.1999).

\section{Figure 61. VPS41 and AP3 mediated transport.}

The AP-3-dependent ALP pathway bypasses the endosome and is independent of most CPY pathway components. Both of the known protein cargoes of pathway-ALP and Vam3contain di-leucine sorting signals, which direct their sorting into AP-3-coated vesicles that form at the trans-Golgi. Formation of these AP-3-coated vesicles, but not AP-3 association with this compartment, requires the function of Vps41. Vam3 is required at the vacuole for fusion of ALP pathway intermediates. Inhibition of the fusion reaction in vam $3{ }^{t s f}$ cells leads to accumulation of AP-3-coated ALP pathway intermediates (Peter rehling.1999).

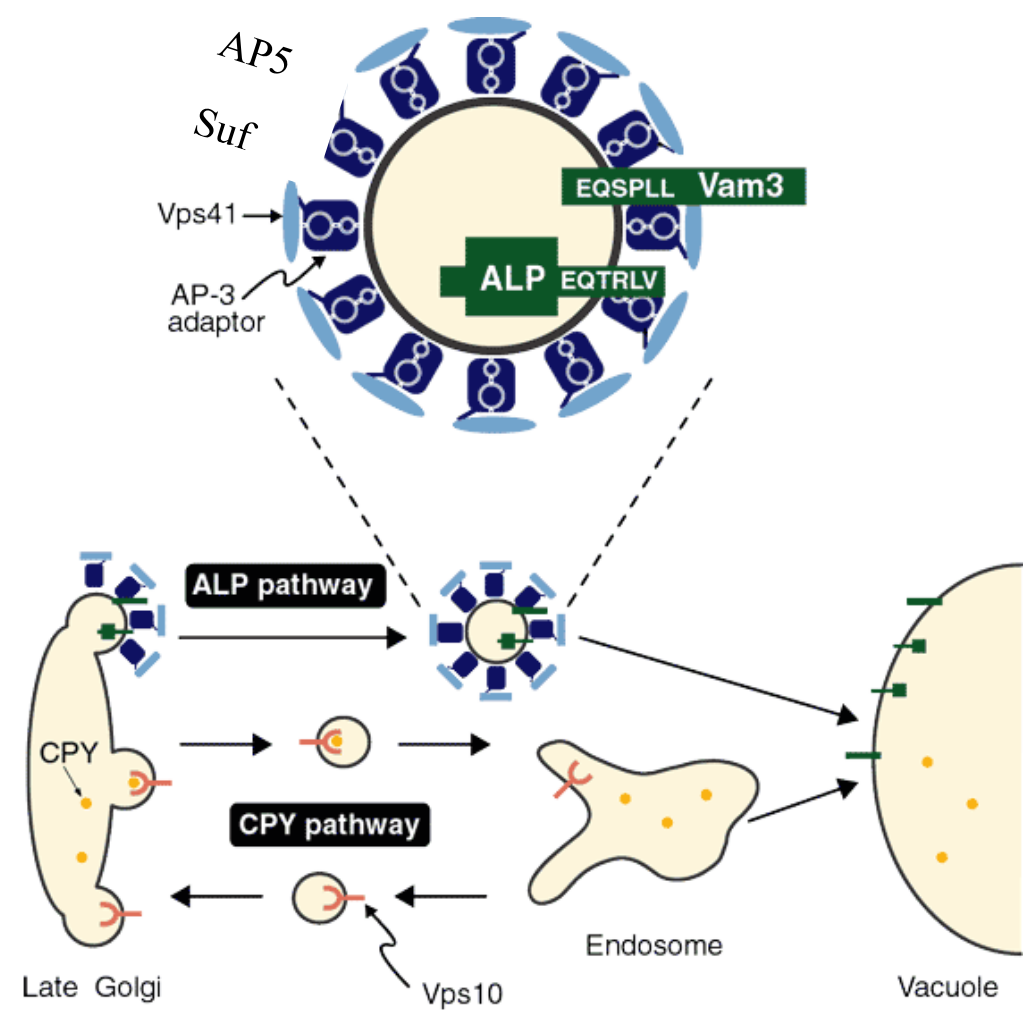

Suf mutants showing less LAMP carrier intermediates suggest that suf might be necessary to make LAMP carriers. Remarkably, the suf mutant has more AP3 on secretory granule and less intermediate vesicle while in wildtype there are more intermediate vesicle 
and very less in secretory granule indicating that the AP3 positive vesicle did not leave from secretory granule in suf mutant. Surprisingly, the presence of VPS41 positive vesicles are strongly reduced in suf mutant suggesting that the accumulation of AP3 on secretory granule is because of absence of VPS41, since VPS41 is necessary to make AP3 vesicle from secretory granule. Absence of VPS41 on secretory granule reduces AP3 intermediate vesicle and accumulate AP3 on secretory granule as shown by previous studies (Peter rehling.1999). VPS41 has been recently shown to play as coat protein for AP3 vesicles apart from their sorting and fusion role. The study showed that VPS41 with its clathrin heavy chain domain makes clathrin like coat for AP3 vesicles to facilitate formation of large dense core vesicle (LDCV) for regulated secretory pathway (Asensio et al.,2013). In neuro-endocrine PC12 cells, loss of AP-3 disrupts sorting to the regulated secretory pathway, dis regulates the release of soluble cargo, and indeed impairs the formation of LDCVs (Asensio et al.,2010) Reduced in number and larger than normal, LDCVs still form in the absence of AP-3, but show different composition (such as the calcium sensor synaptotagmin) required for regulated exocytosis (Asensio et al., 2010). AP-3 thus directs formation of the regulated secretory pathway. So, VPS41 is necessary to form AP3 vesicle, essential to form DCV in regulated secretory pathway with AP3 and function in lysosomal fusion as HOPS complex. Thus, the role of VPS41 in different places almost mimics the defect in suf mutant oocyte, fragmented lysosome suggesting defect in fusion, defect in DCV formation in regulated secretory pathway and reduced AP3 positive LAMP carrier showing that suf is necessary in LAMP carrier pathway.

The bioinformatics prediction shows that suf has domain for clathrin heavy chain (CHC) and beta coatomer domain of COPI which forms the clathrin like coat in VPS41. Interestingly, another study also predicted that suf might act as a coat protein (Hirst et al.,2013a). Although CHC can form coat like clathrin in VPS41, further structural study has to be done to confirm the coat formation in suf and its exact mechanism. Does suf and VPS41 act together in forming coat or function independently, remains elusive. Nevertheless, the failure of VPS41 recruitment on secretory granule suggests that suf is necessary to recruit VPS41 on secretory granule membrane. Because, in the suf mutant VPS41 localization is strongly reduced on secretory granule, which affects the formation of AP3 vesicle. Still LAMP1, VPS41 and AP3 reaches lysosome in suf mutant as it is shown before, it only delay or reduces but do not abolish the LAMP transport suggesting multiple route for LAMP1 cargos. 


\section{Discussion}

Although few studies suggests its role of AP5 in lysosomal sorting, the origin of the cargo is not known, as AP5 localization is not clear. Since it interacts with suf and if suf plays with VPS41 and AP3 to sort cargo, where does AP5 interact with suf? Does suf uses AP5 as an adaptor while VPS41 uses AP3 as an adaptor, alternatively suf uses AP5 as an adaptor for cargo sorting and a defect in this pathway hinders the sorting of AP3 vesicle with VPS41. This reasoning is because both have a similar role and might be involved in sorting related cargos. Interestingly, VPS41, Suf, AP3 and AP5 mutation causes HSP in human. The vesicle formation starts with receptor binding to cargo and adaptor complex, which then recruit the coat to complete the budding with fission machinery (Fig. 62). Biochemical analysis on AP5 and suf would unearth their interactions with the adaptor, cargo (Cathepsins) and receptor for sorting.

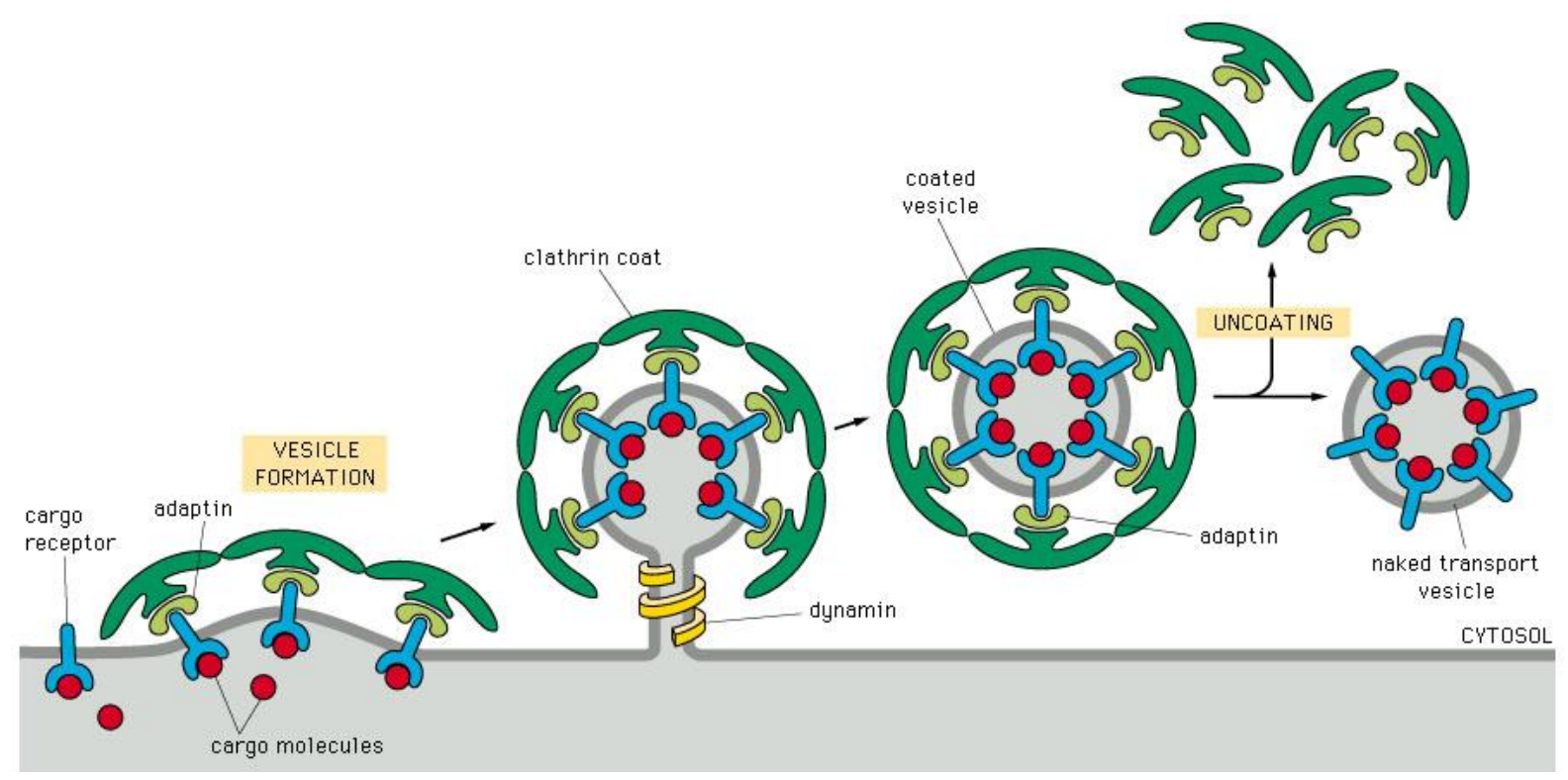

\section{Figure 62. Sorting and budding of new vesicle.}

The sorting of a cargo onto a vesicle requires receptor binding to cargo and an adaptor followed by the coat protein which might be clathrin or some other coat to complete the fission (http://animalcellbiology.wordpress.com).

Recently, a study used RNAi screen to identify the role for hetero tetrameric adaptor protein AP-3 in regulated secretion and in particular, LDCV formation. They found that depletion of AP3 causes defect in regulated secretory pathway. Indeed, mocha mice lacking AP-3 have a severe neurological and behavioral phenotype, but this has been attributed to a role for AP-3 in the endolysosomal rather than the biosynthetic pathway. 
But, they found that adrenal chromaffin cells from mocha animals show increased constitutive exocytosis of both soluble cargo and LDCV membrane proteins, reducing the response to stimulation (Sirkis et al.,2013).

\subsection{Mitochondrial defect in suf mutant}

The current study finds that the suf mutant accumulates more mitochondria and abnormal distribution. Suf has been shown to localize on mitochondrial membrane although the function is still not clear. Mitochondrial fission and lipid molecules transport is dependent on the physical contact with ER using complex called ERMES, so the defect in this connection also might cause defect in mitochondria (Rowland and Voeltz.2012). Recent study also showed that the mitochondria connected physically to lysosome for lipid transport via vCLAMP complex and both ER and Lysosome connection to mitochondria is co-regulated (Fig. 63 a), since removal of one connection is compensated by increasing the connection area with another. Removal of both connections is lethal to the cell indicating their necessity for cell survival (Elbaz-Alon et al.,2014; Honscher et al.,2014). Hence, the defect in the mitochondria in suf mutant might be because of the non-functional lysosome.

Mitochondrion is very sensitive organelle, which also indicates the state of the health of the cell. Any defect in mitochondria leads to multiple diseases including neuron degeneration in many different mutations (Rawson et al.,2014). Transport of mitochondria in neuron is also crucial and defect in transport would cause accumulation and results in neuron degeneration. Furthermore, mitochondrial biogenesis and their fission-fusion process have more similarity to vesicular membrane fission and fusion process. So, defect in the lysosome and ER biogenesis and their function will affect mitochondrial functions. The Mitochondrial defect also causes HSP in human (Schapira.2006; Sheng and Cai.2012; Archer.2013; Noreau et al.,2014).

a

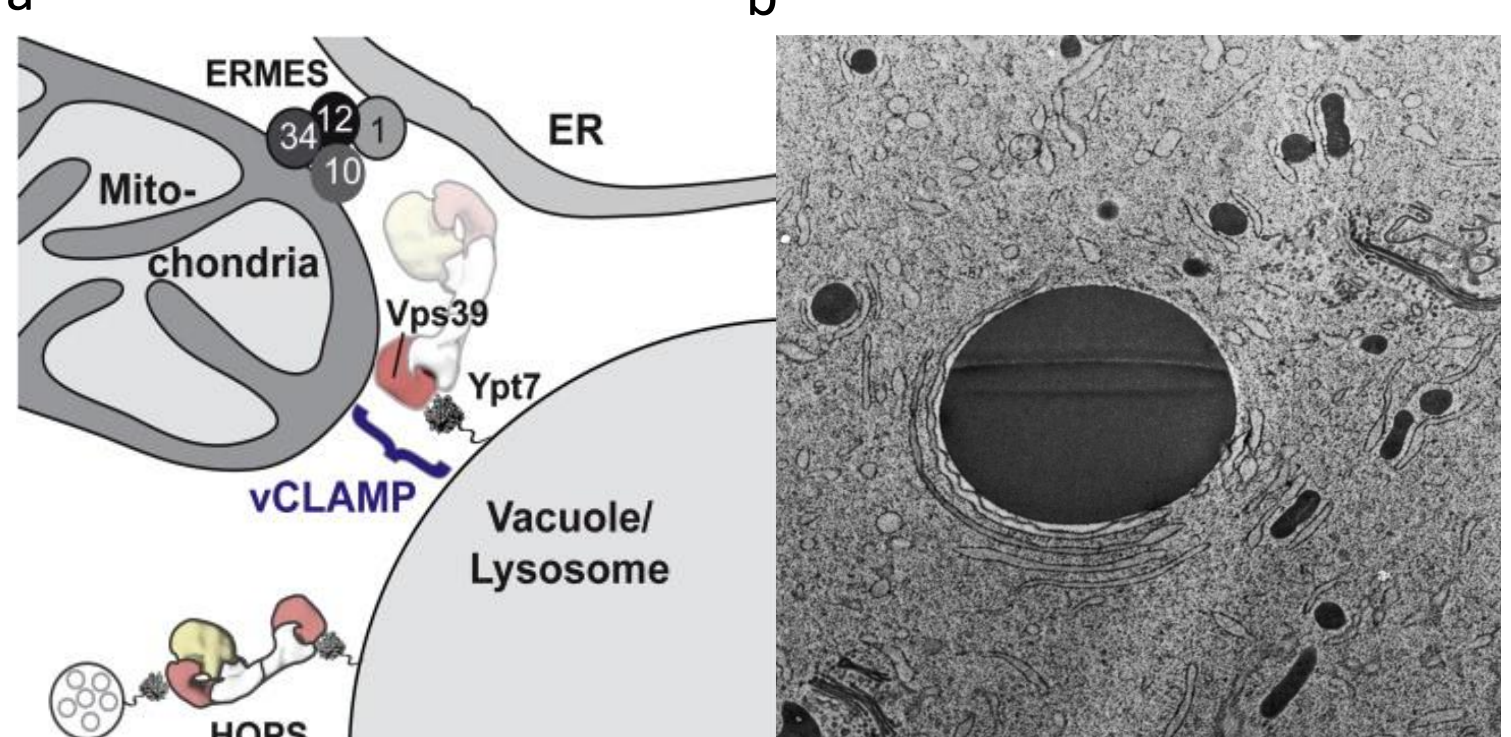




\section{Figure 63. Mitochondrial connections to lysosome and ER.}

Cartoon shows the connection of lysosome and ER with mitochondria wit involved known molecule. b. Electron micrograph of zebrafish oocyte showing dark yolk globule/lysosome connected to ER and ER connection to mitochondria.

Mitochondrial defect in suf mutant might be direct based on its localization but suf function in mitochondria is not clearly known. Interestingly, one of the other opaque egg mutant ruehrei has mutation in TOMM70a, (mitochondrial outer membrane receptor). ruehrei egg shows defect in the lysosome and display fragmented lysosomes like the suf mutant indicating the connection between lysosome defect in suf mutant and mitochondria. In zebrafish oocyte, we also notice strong connection of ER with lysosome and mitochondria (Fig.63 b). Does TOMM70a have any role in lysosome-mitochondrial connection? In addition, why mutation in TOMM70a causes fragmented lysosome is unclear and study on ruehrei will help to understand the role of suf on lysosome and mitochondria.

\subsection{Suf mutation causes HSP in human}

HSP is a heterogenetic group of motor neuron degenerative disease in human. It is a progressive neuron degenerative disease and shows multiple symptoms in brain, spasticity in lower limb and mental disorder. Until now, the possible hypothesis for causing this disease is the defect in long motor neuronal connection to lower neurons, which retract their connections at the later stages. The human patients do not show any developmental defect consistent with fish and mouse models (Khundadze et al.,2013; Kanagaraj et al.,2014). In humans, Suf/Spastizin encodes one of the more than 54 loci involved in the neurodegenerative disorder HSP. The mutated genes encode regulators of diverse cellular processes (Blackstone.2012) with many different genes, but most of the animal model and cell culture studies show a defect in the lysosome including the current study. However, the origin of the defect is not characterized since the function of those genes are unknown or even for known genes the connection to lysosome has not yet been made. Present study shows the genetic evidence for the defect in lysosome and secretory granule maturation for 
the first time, which was never considered in HSP. Interestingly, defects in regulated secretion may explain some of the HSP symptoms. Cortical granules appear identical to large dense-core vesicles (LDCV)(Arvan and Castle.1998; Tooze et al.,2001; Meldolesi et al.,2004; Kim et al.,2006), which store neurotropic factors at the synapse(Poo.2001). Neurotropic factors are responsible for the dynamics and maintenance of synaptic connections (Minichiello.2009; Caroni et al.,2012). In contrast to neurotransmitter vesicles, DCV needs to be transported from the cell body to the synapse. Synaptic vesicles are used to make connections with other neurons but to maintain a strong connection stronger for a long time they need dense core vesicles. If there is a defect in dense core vesicle then they withdraw their connections, referred as 'dying back' symptom. Dying back symptom was hypothesized as a main symptom in HSP while the cell body is still active; suggesting that DCV function might be necessary to keep the long motor neuron connection and defect in this will cause HSP symptoms (Caroni et al., 2012). The DCV formation requires proper removal of endolysosomal cargos using VPS41, AP3, AP5 and Suf, if these proteins are not functional in the cell, there will be a defect in the lysosome since the cargo will not be delivered (Hirst et al.,2013a; Khundadze et al.,2013; Renvoise et al.,2014). The current study proposes that the lysosomal defect in suf might be because of the defect in sorting in secretory pathway, which affects both lysosome and DCV formation. This model would resolve why in HSP preferentially the longest axons first degenerate from their synapses. In addition, a defect in long-term potentiation would explain, why HSP phenotypes become apparent in juveniles and adults, but not in embryos.

Interestingly, these mutants also do not show any defect in any other cells or tissue except motor neuron. The current study also observed that suf adult fish do not show any defect but the oocytes from those mothers show a defect consistent with mouse knock out showing only defect in neuron after one year. Although, many studies in cell culture shows defect in lysosome, autophagy, ER and mitochondria but, why the human patient does not show any defect in the development is still unaddressed (Murmu et al.,2011; Renvoise et al.,2014). In zebrafish it has been shown that the spastizin and spatacsin required for motor neuron axonal outgrowth during development which might show the role of these proteins but does not explain the progressive degeneration symptom in human patients (Martin et al.,2012). Still why the oocyte shows specific symptom is unclear, except that they are very active and require fast endocytosis during oogenesis. If, the efficiency of trafficking is the main reason for showing a defect in motor neuron, zebrafish oocyte will explain the role of those genes with their high efficient endocytosis during oogenesis. Investigating the 
molecular network regulated by Suf/Spastizin in zebrafish oocytes as well as transferring these results to the nervous system as described for Atlastin (SPG3A) in zebrafish (Fassier et al.,2010) and Drosophila (Orso et al.,2009) could provide novel insights into the biochemical etiology of HSP and bring us closer to a therapeutic treatment for patients.

\subsection{Proposed model for Suf functions in secretory granule and lysosome}

Suf mutants accumulates dense core vesicle without dense core and fragmented lysosomes. Functionally they fail to degrade the yolk in lysosome and fail to exocytose during chorion elevation. The current study further showed that the lysosomal enzymes accumulate in secretory granule and secretory granule shows defect in sorting and fission. The sorting event in immature secretory granule is necessary to remove lysosomal enzymes, other cargos like lysosomal SNAREs, VAMP4 and Syntaxin by AP1, GGA1, AP3-VPS41 mediated transport to lysosome. Interestingly, the current study showed that suf is necessary for VPS41-AP3 mediated lysosomal transport. As prediction reveals that Suf has CHC domain like VPS41, it might act as coat protein and use AP5 as adaptor complex. Depletion of any components causes defect in dense core vesicle formation and defective in lysosomal enzymes sorting and function. In suf mutant loss of dense core and defect in sorting lysosomal enzymes explains its role in separating lysosomal vesicle from secretory pathway and causes the fragmented lysosome (Fig. 64 a). VPS41 forms clathrin like coat in vitro and in vivo. In immature secretory granules the proteins aggregate under low $\mathrm{pH}$ regulated by vATPase and the soluble cargos are sorted out by receptors then binding to an adaptor proteins, finally recruiting the coat. In VPS41 pathway, VPS41 act as coat and AP3 is an adaptor. Suf can use AP5 as an adaptor for sorting (Fig. 64 b). If suf act as a coat as proposed along with AP5 as an adaptor, then how suf is involved in AP3VPS41 mediated vesicle formation needs to be understood.

\section{Figure 64. Proposed model for suf function}

a. Cartoon shows the defect in suf mutant and red lines indicates the block in suf mutant. b. Proposed role for suf in sorting lysosomal cargo with or similar to VPS41-AP3 pathway. AP3-VPS41 vesicle in blue sorted to lysosome, vesicle in black sorted by suf-AP5 to lysosome. The vesicle in red in between DCV and Lysosomal vesicle shows the constitutive secretion vesicle, which originates from both ISG, further defective in sorting results in change of regulated secretion and lysosomal vesicle into constitutive secretion. 
The arrow with question mark indicates the unknown connection between Suf- AP5 and VPS41-AP3 vesicle transport.

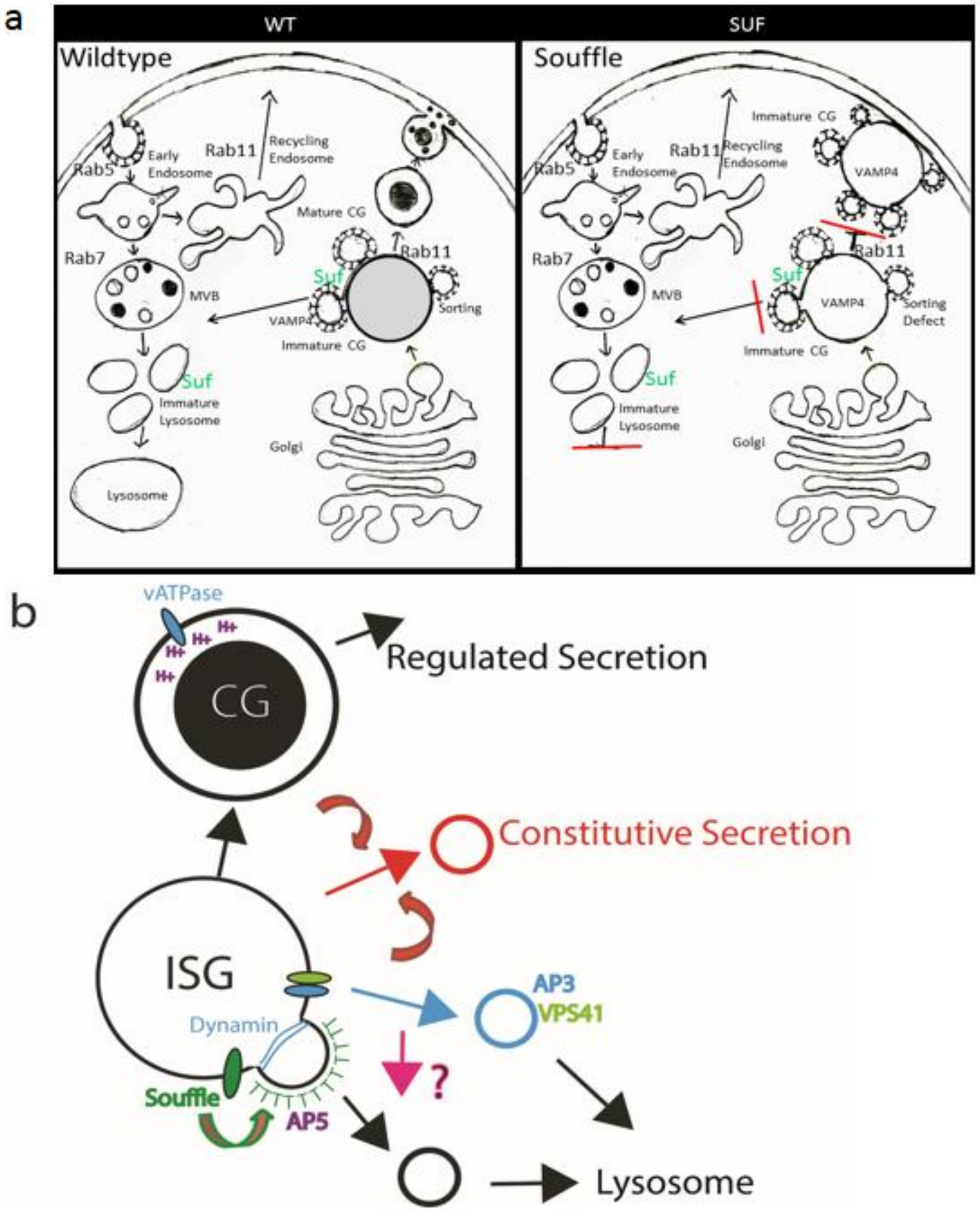

\subsection{Opaque egg mutants might provide as a novel HSP candidate genes.}

As previously described, there are four mutants showing the opaque egg phenotype and shows fragmented lysosome. Newly discovered TOMM70a mutation ruehrei mutant has been predicted as HSP genes in a recent study using interactome of known HSP genes and further studies showed Suf, AP5, AP3, VPS41 and Dynamin in the list of HSP genes, which are found defective in suf mutants. In addition, they validated some of them in 
zebrafish (Novarino et al.,2014). Interestingly in the huge list of possible candidate genes, M6PR and sortilin were absent indicating the unique role for these mutated proteins.

The other opaque egg mutants would provide as novel candidate genes for HSP and might function together with suf or in a pathway since all shows same phenotype. It is also possible that they are AP5 subunits or receptor for suf mediated pathway. With the huge list of known genes mutated in patients but struggling to study their function and disease causing defect without a genetic model, these genetic mutants with the visible phenotype would provide as tool to study further if they will be discovered as HSP genes. Also these groups of mutant would explain more about their function in lysosomal biogenesis and elucidate the role of suf in secretory granule maturation as well.

\subsection{Suf mutant is fitting model for HSP and vesicle trafficking}

HSP is a progressive neuron degenerative disease in human. They show only symptom in the motor neuron and it is an age dependent. This tissue and time dependent phenotype makes it difficult to make an animal model or to use tissue culture to study the functions of these mutated genes to understand the disease pathology. Even the fibroblast from the patient does not show any defect consistent with their disease symptom. Further, even animal models mutated for these genes do not show any symptom during development, which again creates problem to study HSP.

suf mutant which was discovered in a forward genetic screen shows this specific phenotype in the oocyte. This mutation in zebrafish is very similar to mutation in human HSP patients as reported by Hanein et al., 2008. Still it is unclear why the oocyte is very sensitive for this mutation like motor neuron in humans affected by HSP. However, this gives an opportunity to study the role of suf function in trafficking and to understand the HSP disease. Zebrafish oocyte with its huge vesicle size (Lysosome: 5-6 $\mu \mathrm{m}$ and secretory vesicle: 5-6 $\mu \mathrm{m}$ ) and very active and efficient for vesicle trafficking like long neurons provides fitting system to study these genes (Fig.65). Furthermore, zebrafish also provide many possible ways for making mutants and other genetic and cell biological studies. Thus, suf mutant oocytes are suitable model for investigating genes involved in HSP disease. 


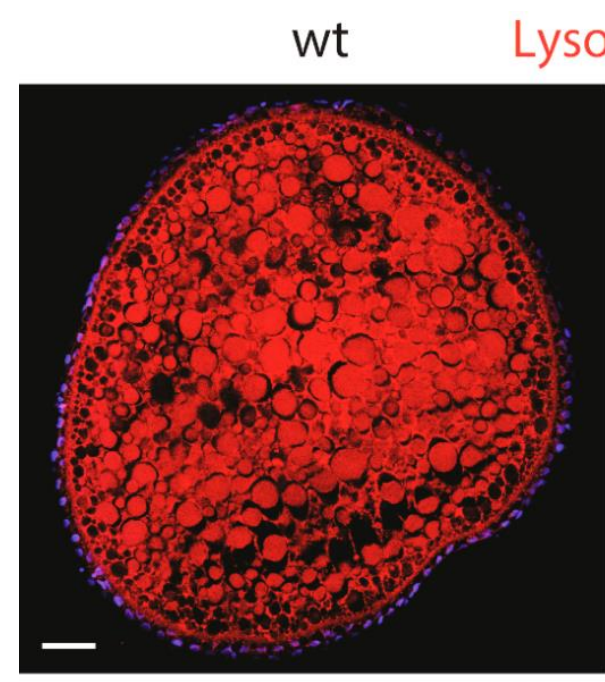

\section{suf}
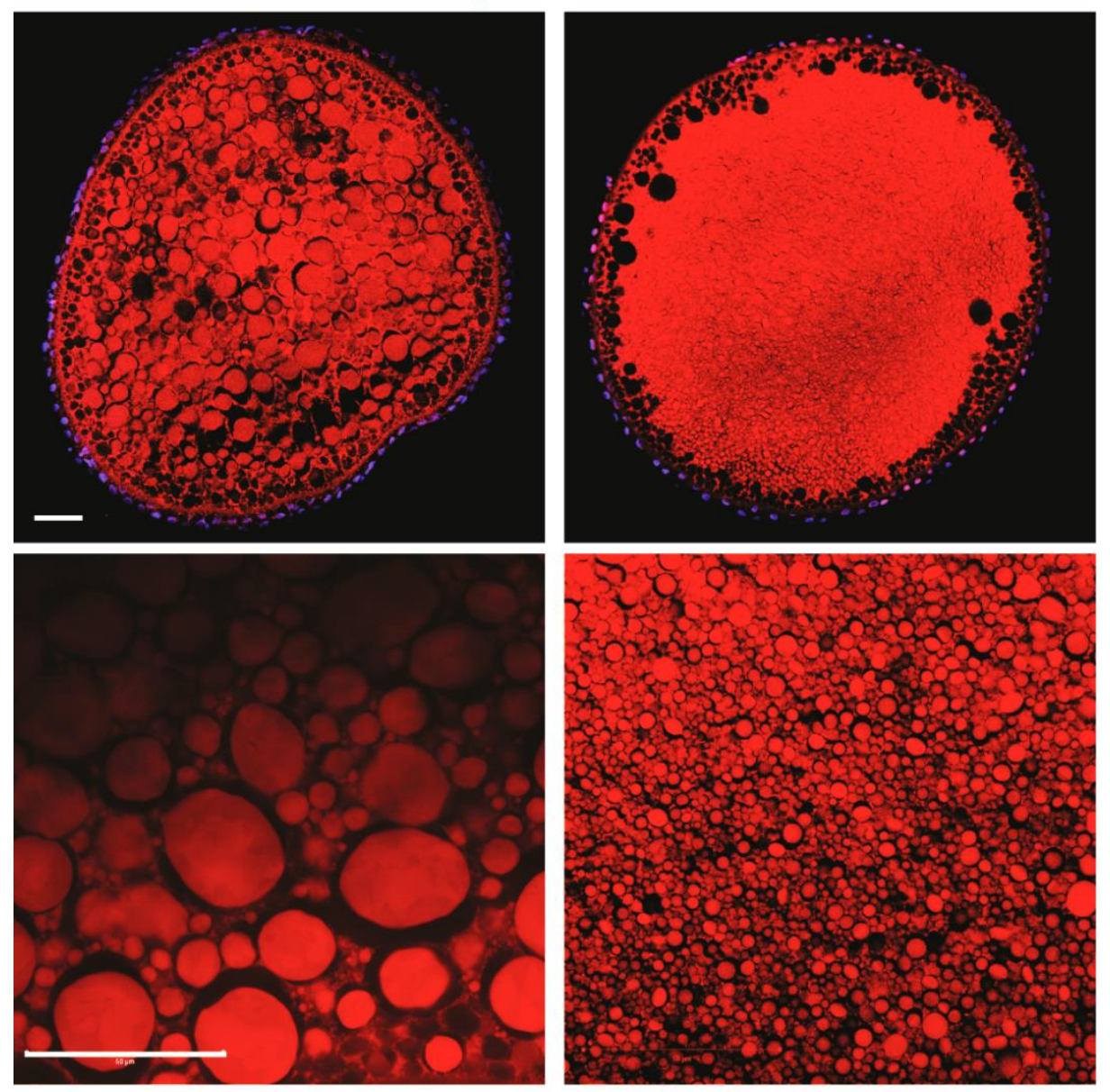

Figure 65. Suf mutant oocyte is a fitting model for HSP.

Staining for lysosome in red and follicle cell nuclei in blue. Left panel wt and right panel from mutant. Notice the huge lysosome also secretory vesicle in the cortex of the oocyte in the top panel (Black circle hole). Sclale-50 $\mu \mathrm{m}$.

\subsection{Prospective}

The present study proposes a role for suf as a coat protein during sorting in secretory granule, it is necessary to find the receptor for sorting and the adaptor protein. Since it interacts with AP5, it could be that AP5 is an adaptor for suf but it is essential to analyze further on reduction in AP3 vesicles in suf mutant. Further study would be required on interaction between suf and VPS41 to explain whether they work together or parallel. Finally, understanding the other opaque egg mutant's role in lysosome biogenesis and secretion will help to understand HSP disease better in humans and shed lights on therapeutic strategies for patients. 


\section{Conclusions}

The current study is aimed at identifying novel regulators of oogenesis in vertebrate. A group of opaque egg mutants was discovered in a maternal screen in zebrafish. The present study takes the advantage of genetics to study one of the mutant called soufflé. The gene was positionally cloned as zfyve26/spastizin and preliminary studies showed that these mutants have a defect in endolysosomal pathway. Therefore, the aim was set to study soufflé role in endosomal trafficking during oogenesis.

Endosomal analysis revealed that suf mutant has a defect in lysosomal function with fragmented lysosome and rab11 postive vesicle. In-vivo transferrin assay showed that the recycling is not affected. Immunostaining analysis showed that the rab11 positive vesicle is secretory granule and suf mutant accumulate immature secretory granule. Electron microscopy studies revealed that the dense core vesicle formation is affected and immature secretory granule failed in sorting and budding of the vesicle. In-vivo functional assay confirmed that the secretory granules are not functional and they fail to elevate the chorion after fertilization. Moreover, F actin staining showed that they failed in exocytosis since the vesicle is not matured. Dynamin inhibition in wild type phenocopies the suf mutant functionally and at cellular level. In summary, suf is essential for secretory granule maturation to form dense core vesicle successfully.

The fragmented lysosome and missing of lysosomal enzymes cathepsin explains the opaque phenotype. The lysosomal transport pathways mediated by M6PR and Sortilin are not affected but AP3 positive LAMP carrier intermediates were reduced while AP3 accumulate more in secretory granule. AP3 vesicle requires VPS41 as a coat and they are reduced on secretory granule in suf mutant, suggesting that suf is needed to recruit VPS41 on secretory granule. Suf with clathrin heavy chain motif could act as a coat protein like VPS41. Suf interaction with AP5 supports further that it might be a coat protein for AP5. But why AP3 vesicles are affected still need further investigation. It could be that the sorting defect causes both defect or suf and VPS41 dually act as a coat for both AP5 and AP3. In conclusion, Suf is necessary for sorting at immature secretory granule level during secretory granule maturation to form dense core vesicles and transport the lysosomal cargo with VPS41 as a coat protein.

Interestingly, function of suf in dense core vesicle formation suggests the role in neuronal transmission and the reason for HSP. The dense core vesicle are necessary to 
maintain the neuronal connection for a longer time but, in their absence the previously made connection will start to die back, which is one of the symptom of HSP. This made the suf mutant a suitable model to study the HSP genes and to understand the disease pathology. 


\section{Bibliography}

Abigail Diaz-Tellez CZ, Samantha Carrillo-, Rosas, Jose L. Ramos-Balderas, Maldonado E. 2012. Scarb2a is required for Brain and Notochord development in zebrafish. International meeting on ebrafish development and genetics-abstract book: 130.

Ahn HJ, Park Y, Kim S, Park HC, Seo SK, Yeo SY, Geum D. 2010. The expression profile and function of Satb2 in zebrafish embryonic development. Molecules and cells 30: $377-382$.

Archer SL. 2013. Mitochondrial dynamics--mitochondrial fission and fusion in human diseases. The New England journal of medicine 369: 2236-2251.

Arvan P, Castle D. 1998. Sorting and storage during secretory granule biogenesis: looking backward and looking forward. Biochem J 332 ( Pt 3): 593-610.

Asensio CS, Sirkis DW, Edwards RH. 2010. RNAi screen identifies a role for adaptor protein AP-3 in sorting to the regulated secretory pathway. The Journal of cell biology 191: 1173-1187.

Asensio CS, Sirkis DW, Maas JW, Jr., Egami K, To TL, Brodsky FM, Shu X, Cheng Y, Edwards RH. 2013. Self-assembly of VPS41 promotes sorting required for biogenesis of the regulated secretory pathway. Developmental cell 27: 425-437.

Babin JCaL. 2007a. The fish oocyte from basic to biotechnological approach

-. 2007b. The fish oocyte from basic to biotechnological approach

Balklava Z, Pant S, Fares H, Grant B. 2007. Genome-wide analysis identifies a general requirement for polarity proteins in endocytic traffic. Nature Cell Biology 9: 10661073.

Barr FA, Gruneberg U. 2007. Cytokinesis: placing and making the final cut. Cell 131: 847860. 
Becker KA, Hart NH. 1999. Reorganization of filamentous actin and myosin-II in zebrafish eggs correlates temporally and spatially with cortical granule exocytosis. J Cell Sci 112 ( Pt 1): 97-110.

Bembenek JN, Richie CT, Squirrell JM, Campbell JM, Eliceiri KW, Poteryaev D, Spang A, Golden A, White JG. 2007. Cortical granule exocytosis in C. elegans is regulated by cell cycle components including separase. Development 134: 3837-3848.

Bembenek JN, White JG, Zheng Y. 2010. A role for separase in the regulation of RAB-11positive vesicles at the cleavage furrow and midbody. Current biology : $C B$ 20: $259-264$

Benli M, Doring F, Robinson DG, Yang X, Gallwitz D. 1996. Two GTPase isoforms, Ypt31p and Ypt32p, are essential for Golgi function in yeast. EMBO J 15: 64606475 .

Blackstone C. 2012. Cellular pathways of hereditary spastic paraplegia. Annual review of neuroscience 35: 25-47.

Bonifacino JS, Rojas R. 2006. Retrograde transport from endosomes to the trans-Golgi network. Nature reviews Molecular cell biology 7: 568-579.

Bontems F, Stein A, Marlow F, Lyautey J, Gupta T, Mullins MC, Dosch R. 2009. Bucky ball organizes germ plasm assembly in zebrafish. Current biology : $C B$ 19: 414 422.

Briguglio JS, Kumar S, Turkewitz AP. 2013. Lysosomal sorting receptors are essential for secretory granule biogenesis in Tetrahymena. The Journal of cell biology 203: 537550 .

Bukoreshtliev NV, Hodneland E, Eichler TW, Eifart P, Rustom A, Gerdes HH. 2012. Partitioning and Exocytosis of Secretory Granules during Division of PC12 Cells. International journal of cell biology 2012: 805295.

Burd CG, Emr SD. 1998. Phosphatidylinositol(3)-phosphate signaling mediated by specific binding to RING FYVE domains. Molecular cell 2: 157-162. 
carnevali BaS. 1999. <Molecular cloning and expression of ovarian cathepsin D in seabream, Sparus aurata.pdf $>$.

Carnevali O, Cionna C, Tosti L, Lubzens E, Maradonna F. 2006. Role of cathepsins in ovarian follicle growth and maturation. General and comparative endocrinology 146: $195-203$.

Caroni P, Donato F, Muller D. 2012. Structural plasticity upon learning: regulation and functions. Nat Rev Neurosci 13: 478-490.

Chen C, Garcia-Santos D, Ishikawa Y, Seguin A, Li L, Fegan KH, Hildick-Smith GJ, Shah DI, Cooney JD, Chen $\mathrm{W}$ et al. 2013. Snx3 regulates recycling of the transferrin receptor and iron assimilation. Cell metabolism 17: 343-352.

Chen W, Feng Y, Chen D, Wandinger-Ness A. 1998. Rab11 is required for trans-golgi network-to-plasma membrane transport and a preferential target for GDP dissociation inhibitor. Mol Biol Cell 9: 3241-3257.

Cheng H, Govindan JA, Greenstein D. 2008. Regulated trafficking of the MSP/Eph receptor during oocyte meiotic maturation in C. elegans. Curr Biol 18: 705-714.

Chiara Vantaggiato EC, Maria Teresa Bassi. 2013. ZFYVE26/SPASTIZIN: A close link between complicated hereditary spastic paraparesis and autophagy. autophagy.

Clelland ES, Tan Q, Balofsky A, Lacivita R, Peng C. 2007. Inhibition of premature oocyte maturation: a role for bone morphogenetic protein 15 in zebrafish ovarian follicles. Endocrinology 148: 5451-5458.

Cowles GaSE. 1997. <AP3 complex is essential for Cargo-Selective Transport to the Yeast Vacuole.pdf>.

DEREK C. RADISKY WBS, SCOTT D. EMR, AND JERRY KAPLAN. 1997. <PNAS1997-Radisky-5662-6 Characterization of VPS41, a gene required for vacuolar .trafficking in yeast.pdf $>$.

Dettmer J, Hong-Hermesdorf A, Stierhof YD, Schumacher K. 2006. Vacuolar H+-ATPase activity is required for endocytic and secretory trafficking in Arabidopsis. The Plant cell 18: 715-730. 
Dosch R, Wagner DS, Mintzer KA, Runke G, Wiemelt AP, Mullins MC. 2004. Maternal control of vertebrate development before the midblastula transition: mutants from the zebrafish I. Developmental cell 6: 771-780.

Dower WJ, Miller JF, Ragsdale CW. 1988. High efficiency transformation of E. coli by high voltage electroporation. Nucleic Acids Res 16: 6127-6145.

Eaton BA, Haugwitz M, Lau D, Moore HP. 2000. Biogenesis of regulated exocytotic carriers in neuroendocrine cells. J Neurosci 20: 7334-7344.

El-Jouni W, Haun S, Hodeify R, Walker AH, Machaca K. 2007. Vesicular traffic at the cell membrane regulates oocyte meiotic arrest. Development 134: 3307-3315.

Elbaz-Alon Y, Rosenfeld-Gur E, Shinder V, Futerman AH, Geiger T, Schuldiner M. 2014. A dynamic interface between vacuoles and mitochondria in yeast. Developmental cell 30: 95-102.

Ellis K, Bagwell J, Bagnat M. 2013. Notochord vacuoles are lysosome-related organelles that function in axis and spine morphogenesis. The Journal of cell biology 200: 667-679.

Fabra M, Raldua D, Bozzo MG, Deen PM, Lubzens E, Cerda J. 2006. Yolk proteolysis and aquaporin-1o play essential roles to regulate fish oocyte hydration during meiosis resumption. Dev Biol 295: 250-262.

Fassier C, Hutt JA, Scholpp S, Lumsden A, Giros B, Nothias F, Schneider-Maunoury S, Houart C, Hazan J. 2010. Zebrafish atlastin controls motility and spinal motor axon architecture via inhibition of the BMP pathway. Nat Neurosci 13: 1380-1387.

Ferguson SM, De Camilli P. 2012. Dynamin, a membrane-remodelling GTPase. Nat Rev Mol Cell Biol 13: 75-88.

Gaullier JM, Simonsen A, D'Arrigo A, Bremnes B, Stenmark H, Aasland R. 1998. FYVE fingers bind PtdIns(3)P. Nature 394: 432-433.

Goldstein JL, Brown MS, Anderson RG, Russell DW, Schneider WJ. 1985. Receptormediated endocytosis: concepts emerging from the LDL receptor system. Annu Rev Cell Biol 1: 1-39. 
Gonzalez-Jamett AM, Momboisse F, Haro-Acuna V, Bevilacqua JA, Caviedes P, Cardenas AM. 2013. Dynamin-2 function and dysfunction along the secretory pathway. Frontiers in endocrinology 4: 126.

Grant B, Hirsh D. 1999. Receptor-mediated endocytosis in the Caenorhabditis elegans oocyte. Molecular biology of the cell 10: 4311-4326.

Gretchen Dollar ES, Jason Michaud and Robert S. Cohen. 2002. Rab11 polarization of the Drosophila oocyte a novel link between membrane trafficking, microtubule organization, and oskar mRNA localization and translation.pdf. Development.

Groh KJ, Schonenberger R, Eggen RI, Segner H, Suter MJ. 2013. Analysis of protein expression in zebrafish during gonad differentiation by targeted proteomics. Gen Comp Endocrinol 193: 210-220.

Hanein S, Martin E, Boukhris A, Byrne P, Goizet C, Hamri A, Benomar A, Lossos A, Denora P, Fernandez $\mathbf{J}$ et al. 2008. Identification of the SPG15 gene, encoding spastizin, as a frequent cause of complicated autosomal-recessive spastic paraplegia, including Kjellin syndrome. American journal of human genetics $\mathbf{8 2}$ : 992-1002.

Hannemann M. 2012. Dense core vesicle maturation at the golgi-endosome interface in C.elegans. PhD Thesis, Goettingen University, Germany.

Hannemann M, Sasidharan N, Hegermann J, Kutscher LM, Koenig S, Eimer S. 2012. TBC-8, a putative RAB-2 GAP, regulates dense core vesicle maturation in Caenorhabditis elegans. PLoS genetics 8: e1002722.

Harry Towbin TSaJG. 1979. Electrophoretic transfer of proteins from polyacrylamide gels to nitrocellulose sheets procedure and some applications.pdf. PNAS.

Hart NH, Yu SF. 1980. Cortical granule exocytosis and cell surface reorganization in eggs of Brachydanio. J Exp Zool 213: 137-159.

Hehnly H, Doxsey S. 2014. Rab11 endosomes contribute to mitotic spindle organization and orientation. Dev Cell 28: 497-507. 
Hirst J, Barlow LD, Francisco GC, Sahlender DA, Seaman MN, Dacks JB, Robinson MS. 2011. The fifth adaptor protein complex. PLoS Biol 9: e1001170.

Hirst J, Borner GH, Edgar J, Hein MY, Mann M, Buchholz F, Antrobus R, Robinson MS. 2013a. Interaction between AP-5 and the hereditary spastic paraplegia proteins SPG11 and SPG15. Molecular biology of the cell 24: 2558-2569.

Hirst J, Irving C, Borner GH. 2013b. Adaptor protein complexes AP-4 and AP-5: new players in endosomal trafficking and progressive spastic paraplegia. Traffic 14: 153164.

Hoekstra D, Tyteca D, van IJzendoorn SC. 2004. The subapical compartment: a traffic center in membrane polarity development. J Cell Sci 117: 2183-2192.

Honscher C, Mari M, Auffarth K, Bohnert M, Griffith J, Geerts W, van der Laan M, Cabrera M, Reggiori F, Ungermann C. 2014. Cellular metabolism regulates contact sites between vacuoles and mitochondria. Developmental cell 30: 86-94.

Hutton. 1982. <The internal $\mathrm{pH}$ and membrane potential of the insulin-secretory granule.pdf $>$.

Jalabert B, Theron M-C, Heydorff M. 1978. Production of fertilizable oocytes from follicles of rainbow trout (Salmo gairdnerii) following in vitro maturation and ovulation. Ann Biol anim Bioch Biophys 18: 461-470.

Jedd G, Mulholland J, Segev N. 1997. Two new Ypt GTPases are required for exit from the yeast trans-Golgi compartment. J Cell Biol 137: 563-580.

Judith Klumperman RK, Janice Griffith and Peter Arvan. 1998. <J Cell Biol-1998Klumperman-359-71 Mannose 6-Phosphate Receptors Are Sorted from Immature Secretory Granules via Adaptor Protein AP-1, Clathrin, and Syntaxin 6-positive Vesicles.pdf>.

Kakhlon O, Sakya P, Larijani B, Watson R, Tooze SA. 2006. GGA function is required for maturation of neuroendocrine secretory granules. EMBO J 25: 1590-1602. 
Kanagaraj P, Gautier-Stein A, Riedel D, Schomburg C, Cerda J, Vollack N, Dosch R. 2014. Souffle/Spastizin controls secretory vesicle maturation during zebrafish oogenesis. PLoS genetics 10: e1004449.

Karin M, Mintz B. 1981. Receptor-mediated endocytosis of transferrin in developmentally totipotent mouse teratocarcinoma stem cells. J Biol Chem 256: 3245-3252.

Kathryn Ellis BDH, Michel Bagnat. 2013. The vacuole within: How cellular organization dictates notochord function. Bioarchitecture.

Kent HM, Evans PR, Schafer IB, Gray SR, Sanderson CM, Luzio JP, Peden AA, Owen DJ. 2012. Structural basis of the intracellular sorting of the SNARE VAMP7 by the AP3 adaptor complex. Developmental cell 22: 979-988.

Khundadze M, Kollmann K, Koch N, Biskup C, Nietzsche S, Zimmer G, Hennings JC, Huebner AK, Symmank J, Jahic A et al. 2013. A hereditary spastic paraplegia mouse model supports a role of ZFYVE26/SPASTIZIN for the endolysosomal system. PLoS genetics 9: e1003988.

Khvotchev MV, Ren M, Takamori S, Jahn R, Sudhof TC. 2003. Divergent functions of neuronal Rab11b in Ca2+-regulated versus constitutive exocytosis. J Neurosci 23: 10531-10539.

Kienzle C, von Blume J. 2014. Secretory cargo sorting at the trans-Golgi network. Trends in cell biology.

Kim T, Gondre-Lewis MC, Arnaoutova I, Loh YP. 2006. Dense-core secretory granule biogenesis. Physiology 21: 124-133.

Kimmel CB, Ballard WW, Kimmel SR, Ullmann B, Schilling TF. 1995. Stages of embryonic development of the zebrafish. Developmental dynamics 203: 253-310.

Konopka CA, Schleede JB, Skop AR, Bednarek SY. 2006. Dynamin and cytokinesis. Traffic 7: 239-247.

Kouranti I, Sachse M, Arouche N, Goud B, Echard A. 2006. Rab35 regulates an endocytic recycling pathway essential for the terminal steps of cytokinesis. Current biology : CB 16: $1719-1725$. 
Laemmli UK. 1970. Cleavage of structural proteins during the assembly of the head of bacteriophage T4. Nature 227: 680-685.

LaFleur GJ, Jr., Raldua D, Fabra M, Carnevali O, Denslow N, Wallace RA, Cerda J. 2005. Derivation of major yolk proteins from parental vitellogenins and alternative processing during oocyte maturation in Fundulus heteroclitus. Biology of reproduction 73: 815-824.

Lapierre LA, Dorn MC, Zimmerman CF, Navarre J, Burnette JO, Goldenring JR. 2003a. Rab11b resides in a vesicular compartment distinct from Rab11a in parietal cells and other epithelial cells. Experimental cell research 290: 322-331.

Lapierre LA, Dorn MC, Zimmerman CF, Navarre J, Burnette JO, Goldenring JR. 2003b. Rab11b resides in a vesicular compartment distinct from Rab11a in parietal cells and other epithelial cells. Exp Cell Res 290: 322-331.

Le Menn F, Cerda J, Babin PJ. 2007. Ultrastructural aspects of the ontogeny and differentiation of ray-finned fish ovarian follicles. in The Fish Oocyte: From Basic Studies to Biotechnological Applications (eds. PJ Babin, J Cerda, E Lubzens), pp. 137. Springer, Dordrecht, The Netherlands.

Liu M. 2011. The biology and dynamics of mammalian cortical granules. Reprod Biol Endocrinol 9: 149.

Lubzens E, Young G, Bobe J, Cerda J. 2010. Oogenesis in teleosts: how eggs are formed. General and comparative endocrinology 165: 367-389.

Mandel M, Higa A. 1970. Calcium-dependent bacteriophage DNA infection. J Mol Biol 53: $159-162$.

Martin E, Yanicostas C, Rastetter A, Naini SM, Maouedj A, Kabashi E, Rivaud-Pechoux S, Brice A, Stevanin G, Soussi-Yanicostas N. 2012. Spatacsin and spastizin act in the same pathway required for proper spinal motor neuron axon outgrowth in zebrafish. Neurobiology of disease 48: 299-308.

Maxfield Fa. 1994. <J Cell Sci-1994-Fagotto-3325-37 Changes in yolk platelet pH during xenopus development correlate wih yolk utiliation.pdf>. 
Meldolesi J, Chieregatti E, Luisa Malosio M. 2004. Requirements for the identification of dense-core granules. Trends Cell Biol 14: 13-19.

Mikhail Khvotchev R, Shigeo, Reinhard Jahn and Thomas Suedorf. 2003. Divergent functions of neuronal Rab11b in Ca2+-regulated versus constitutive exocytosis.pdf. The journal of Neuroscience.

Minichiello L. 2009. TrkB signalling pathways in LTP and learning. Nat Rev Neurosci 10: 850-860.

Morvan J, Tooze SA. 2008. Discovery and progress in our understanding of the regulated secretory pathway in neuroendocrine cells. Histochemistry and cell biology 129: 243-252.

Mullis K, Faloona F, Scharf S, Saiki R, Horn G, Erlich H. 1986. Specific enzymatic amplification of DNA in vitro: the polymerase chain reaction. Cold Spring Harb Symp Quant Biol 51 Pt 1: 263-273.

Murmu RP, Martin E, Rastetter A, Esteves T, Muriel MP, El Hachimi KH, Denora PS, Dauphin A, Fernandez JC, Duyckaerts C et al. 2011. Cellular distribution and subcellular localization of spatacsin and spastizin, two proteins involved in hereditary spastic paraplegia. Molecular and cellular neurosciences 47: 191-202.

Murray Ba. 2004. Cathepsin L in yolk degradation in c.elegans.

Nagahama Y. 1985. Stimulation of 17 alpha,20 beta-dihydroxy-4-pregnen-3-one production in the granulosa cells of Amago salmon, Oncorhynchus rhodurus, by cyclic nucleotides. The Journal of experimental zoology 236: 371-375.

Nair S, Lindeman RE, Pelegri F. 2013. In vitro oocyte culture-based manipulation of zebrafish maternal genes. Dev Dyn 242: 44-52.

Nakagawa S, Seog, Naoshi, Koji and Hirokawa. 2000a. A Novel Motor, KIF13A, Transports Mannose-6-Phosphate Receptor to Plasma Membrane through Direct Interaction with AP-1 Complex.pdf. Cell. 
Nakagawa s, seog, takio, hirokawa. 2000b. <A Novel Motor, KIF13A, Transports Mannose-6-Phosphate Receptor to Plasma Membrane through Direct Interaction with AP-1 Complex.pdf>.

Neto H, Balmer G, Gould G. 2013. Exocyst proteins in cytokinesis: Regulation by Rab11. Communicative \& integrative biology 6: e27635.

Noreau A, Dion PA, Rouleau GA. 2014. Molecular aspects of hereditary spastic paraplegia. Experimental cell research 325: 18-26.

Novarino G, Fenstermaker AG, Zaki MS, Hofree M, Silhavy JL, Heiberg AD, Abdellateef M, Rosti B, Scott E, Mansour L et al. 2014. Exome sequencing links corticospinal motor neuron disease to common neurodegenerative disorders. Science 343: 506511.

Nüsslein-Volhard C, Dahm R. 2002. Zebrafish - A practical approach. Oxford University Press 261.

Orci L, Ravazzola M, Amherdt M, Louvard D, Perrelet A. 1985. Clathrin-immunoreactive sites in the Golgi apparatus are concentrated at the trans pole in polypeptide hormone-secreting cells. Proc Natl Acad Sci U S A 82: 5385-5389.

Orso G, Pendin D, Liu S, Tosetto J, Moss TJ, Faust JE, Micaroni M, Egorova A, Martinuzzi A, McNew JA et al. 2009. Homotypic fusion of ER membranes requires the dynamin-like GTPase atlastin. Nature 460: 978-983.

Pagni M, Ioannidis V, Cerutti L, Zahn-Zabal M, Jongeneel CV, Hau J, Martin O, Kuznetsov D, Falquet L. 2007. MyHits: improvements to an interactive resource for analyzing protein sequences. Nucleic Acids Res 35: W433-437.

Patki V, Lawe DC, Corvera S, Virbasius JV, Chawla A. 1998. A functional PtdIns(3)Pbinding motif. Nature 394: 433-434.

Pauls S, Geldmacher-Voss B, Campos-Ortega JA. 2001. A zebrafish histone variant H2A.F/Z and a transgenic H2A.F/Z:GFP fusion protein for in vivo studies of embryonic development. Dev Genes Evol 211: 603-610. 
Pelegri F. 2003. Maternal factors in zebrafish development. Developmental dynamics 228: $535-554$

Pelissier A, Chauvin JP, Lecuit T. 2003. Trafficking through Rab11 endosomes is required for cellularization during Drosophila embryogenesis. Curr Biol 13: 1848-1857.

Peter rehling T, David and Scott Emr. 1999. <Formation of AP-3 transport intermediates requires Vps41 function.pdf>.

Pillay CS, Elliott E, Dennison C. 2002. Endolysosomal proteolysis and its regulation. Biochem J 363: 417-429.

Polevoy G, Wei HC, Wong R, Szentpetery Z, Kim YJ, Goldbach P, Steinbach SK, Balla T, Brill JA. 2009. Dual roles for the Drosophila PI 4-kinase four wheel drive in localizing Rab11 during cytokinesis. The Journal of cell biology 187: 847-858.

Pols MS, van Meel E, Oorschot V, ten Brink C, Fukuda M, Swetha MG, Mayor S, Klumperman J. 2013. hVps41 and VAMP7 function in direct TGN to late endosome transport of lysosomal membrane proteins. Nature communications 4: 1361.

Poo MM. 2001. Neurotrophins as synaptic modulators. Nat Rev Neurosci 2: 24-32.

Postlethwait JH. 2007. The zebrafish genome in context: ohnologs gone missing. $J$ Exp Zool B Mol Dev Evol 308: 563-577.

Praefcke GJ, McMahon HT. 2004. The dynamin superfamily: universal membrane tubulation and fission molecules? Nat Rev Mol Cell Biol 5: 133-147.

Radisky S, Scott and Kaplan. 1997. <Characterization of VPS41, a gene required for vacuolar trafficking and high-affinity iron transport in yeast.pdf>.

Raldua f, Maria, Weber and joan cerda. 2006. Cathepsin B mediated yolk degradation in killifish.

Rawson RL, Yam L, Weimer RM, Bend EG, Hartwieg E, Horvitz HR, Clark SG, Jorgensen EM. 2014. Axons degenerate in the absence of mitochondria in C. elegans. Current biology : CB 24: 760-765. 
Reczek D, Schwake M, Schroder J, Hughes H, Blanz J, Jin X, Brondyk W, Van Patten S, Edmunds T, Saftig P. 2007. LIMP-2 is a receptor for lysosomal mannose-6phosphate-independent targeting of beta-glucocerebrosidase. Cell 131: 770-783.

Reichardt I, Stierhof YD, Mayer U, Richter S, Schwarz H, Schumacher K, Jurgens G. 2007. Plant cytokinesis requires de novo secretory trafficking but not endocytosis. Current biology : CB 17: 2047-2053.

Renvoise B, Chang J, Singh R, Yonekawa S, FitzGibbon EJ, Mankodi A, Vanderver A, Schindler A, Toro C, Gahl WA et al. 2014. Lysosomal abnormalities in hereditary spastic paraplegia types SPG15 and SPG11. Annals of clinical and translational neurology 1: 379-389.

Romano M, Rosanova P, Anteo C, Limatola E. 2004. Vertebrate yolk proteins: a review. Molecular reproduction and development 69: 109-116.

Rowland AA, Voeltz GK. 2012. Endoplasmic reticulum-mitochondria contacts: function of the junction. Nature reviews Molecular cell biology 13: 607-625.

Sagona AP, Nezis IP, Bache KG, Haglund K, Bakken AC, Skotheim RI, Stenmark H. 2011. A tumor-associated mutation of FYVE-CENT prevents its interaction with Beclin 1 and interferes with cytokinesis. PloS one 6: e17086.

Sagona AP, Nezis IP, Pedersen NM, Liestol K, Poulton J, Rusten TE, Skotheim RI, Raiborg C, Stenmark H. 2010. PtdIns(3)P controls cytokinesis through KIF13Amediated recruitment of FYVE-CENT to the midbody. Nature cell biology 12: 362371.

Sanger F, Nicklen S, Coulson AR. 1977. DNA sequencing with chain-terminating inhibitors. Proc Natl Acad Sci U S A 74: 5463-5467.

Sathish mallya P, Maria and Lennarz. 1992. <Proteolysis of the major yolk glycoproteins is regulated by acidification of the yolk platelets in sea urchin embryos.pdf>.

Sato M, Grant BD, Harada A, Sato K. 2008. Rab11 is required for synchronous secretion of chondroitin proteoglycans after fertilization in Caenorhabditis elegans. Journal of cell science 121: 3177-3186. 
Schapira AHV. 2006. Mitochondrial disease. The Lancet 368: 70-82.

Schiel JA, Childs C, Prekeris R. 2013. Endocytic transport and cytokinesis: from regulation of the cytoskeleton to midbody inheritance. Trends Cell Biol 23: 319-327.

Schmid SL, Frolov VA. 2011. Dynamin: functional design of a membrane fission catalyst. Annual review of cell and developmental biology 27: 79-105.

Schneider WJ. 1996. Vitellogenin receptors: oocyte-specific members of the low-density lipoprotein receptor supergene family. Int Rev Cytol 166: 103-137.

Schoonderwoert T, Tooze, Martens. 2000. <Inhibition of the vacuolar H+-ATPase perturbs the transport, sorting, processing and release of regulated secretory proteins.pdf>.

Sechi S, Colotti G, Belloni G, Mattei V, Frappaolo A, Raffa GD, Fuller MT, Giansanti MG. 2014. GOLPH3 is essential for contractile ring formation and Rab11 localization to the cleavage site during cytokinesis in Drosophila melanogaster. PLoS genetics 10: e1004305.

Selman K, Petrino TR, Wallace RA. 1994. Experimental conditions for oocyte maturation in the zebrafish, Brachydanio rerio. The Journal of experimental zoology 269: 538550.

Selman wajc. 2001. <2001 Selman et al JEZ-1 Bafilomycin A1 inhibits proteolytic cleavage and hydration but not yolk crystal disassembly or meiosis during maturation of sea bass oocytes..pdf>.

Sharp PA, Sugden B, Sambrook J. 1973. Detection of two restriction endonuclease activities in Haemophilus parainfluenzae using analytical agarose--ethidium bromide electrophoresis. Biochemistry 12: 3055-3063.

Shaw JD, Cummings KB, Huyer G, Michaelis S, Wendland B. 2001. Yeast as a model system for studying endocytosis. Experimental cell research 271: 1-9.

Sheng ZH, Cai Q. 2012. Mitochondrial transport in neurons: impact on synaptic homeostasis and neurodegeneration. Nature reviews Neuroscience 13: 77-93. 
Sigrist CJ, Cerutti L, de Castro E, Langendijk-Genevaux PS, Bulliard V, Bairoch A, Hulo N. 2010. PROSITE, a protein domain database for functional characterization and annotation. Nucleic Acids Res 38: D161-166.

Simon GC, Prekeris R. 2008. Mechanisms regulating targeting of recycling endosomes to the cleavage furrow during cytokinesis. Biochem Soc Trans 36: 391-394.

Sire MF, Babin PJ, Vernier JM. 1994. Involvement of the Lysosomal System in Yolk Protein Deposit and Degradation during Vitellogenesis and EmbryonicDevelopment in Trout. Journal of Experimental Zoology 269: 69-83.

Sirkis DW, Edwards RH, Asensio CS. 2013. Widespread dysregulation of peptide hormone release in mice lacking adaptor protein AP-3. PLoS genetics 9: e1003812.

Sobota JA BN, Eipper BA, Mains RE. 2009. Inhibitors of the V0 subunit of the vacuolar H+-ATPase prevent segregation of lysosomal- and secretory-pathway proteins.

Sorkin A, Von Zastrow M. 2002. Signal transduction and endocytosis: close encounters of many kinds. Nature reviews Molecular cell biology 3: 600-614.

Steegmaier M, Klumperman J, Foletti DL, Yoo JS, Scheller RH. 1999. Vesicle-associated membrane protein 4 is implicated in trans-Golgi network vesicle trafficking. Mol Biol Cell 10: 1957-1972.

Stenmark H, Aasland R, Toh BH, D'Arrigo A. 1996. Endosomal localization of the autoantigen EEA1 is mediated by a zinc-binding FYVE finger. $J$ Biol Chem 271: 24048-24054.

Streisinger G, Walker C, Dower N, Knauber D, Singer F. 1981. Production of clones of homozygous diploid zebra fish (Brachydanio rerio). Nature 291: 293-296.

Studer D, Michel M, Müller M. 1989. High pressure freezing comes of age. Scanning microscopy Supplement 3: 253-268; discussion 268-259.

Taguchi T. 2013. Emerging roles of recycling endosomes. Journal of biochemistry 153: 505-510. 
Takeda K, Cabrera M, Rohde J, Bausch D, Jensen ON, Ungermann C. 2008. The vacuolar V1/V0-ATPase is involved in the release of the HOPS subunit Vps41 from vacuoles, vacuole fragmentation and fusion. FEBS letters 582: 1558-1563.

Tanaka S, Yora T, Nakayama K, Inoue K, Kurosumi K. 1997. Proteolytic Processing of Pro-opiomelanocortin Occurs in Acidifying Secretory Granules of AtT-20 Cells. Journal of Histochemistry \& Cytochemistry 45: 425-436.

Taupenot L, Harper KL, O'Connor DT. 2005. Role of H+-ATPase-mediated acidification in sorting and release of the regulated secretory protein chromogranin A: evidence for a vesiculogenic function. The Journal of biological chemistry 280: 3885-3897.

Tooze J, Tooze SA. 1986. Clathrin-coated vesicular transport of secretory proteins during the formation of ACTH-containing secretory granules in AtT20 cells. J Cell Biol 103: $839-850$.

Tooze SA, Martens GJ, Huttner WB. 2001. Secretory granule biogenesis: rafting to the SNARE. Trends Cell Biol 11: 116-122.

Ullrich O, Reinsch S, Urbe S, Zerial M, Parton RG. 1996. Rab11 regulates recycling through the pericentriolar recycling endosome. J Cell Biol 135: 913-924.

Urbe S, Huber LA, Zerial M, Tooze SA, Parton RG. 1993. Rab11, a small GTPase associated with both constitutive and regulated secretory pathways in PC12 cells. FEBS Lett 334: 175-182.

Vantaggiato C, Crimella C, Airoldi G, Polishchuk R, Bonato S, Brighina E, Scarlato M, Musumeci O, Toscano A, Martinuzzi A et al. 2013. Defective autophagy in spastizin mutated patients with hereditary spastic paraparesis type 15. Brain : $a$ journal of neurology 136: 3119-3139.

von Hofsten J, Olsson PE. 2005. Zebrafish sex determination and differentiation: involvement of FTZ-F1 genes. Reprod Biol Endocrinol 3: 63.

Wallace RA, Selman K. 1990. Ultrastructural aspects of oogenesis and oocyte growth in fish and amphibians. J Electron Microsc Tech 16: 175-201. 
Wang X, Kumar R, Navarre J, Casanova JE, Goldenring JR. 2000. Regulation of vesicle trafficking in madin-darby canine kidney cells by Rab11a and Rab25. The Journal of biological chemistry 275: 29138-29146.

Watanabe Ha. 2010. <secretogranin III.pdf>.

Wessel GM, Brooks JM, Green E, Haley S, Voronina E, Wong J, Zaydfudim V, Conner S. 2001. The biology of cortical granules. Int Rev Cytol 209: 117-206.

Willingham MC, Hanover JA, Dickson RB, Pastan I. 1984. Morphologic characterization of the pathway of transferrin endocytosis and recycling in human KB cells. Proc Natl Acad Sci U S A 81: 175-179.

Wu MM, Grabe M, Adams S, Tsien RY, Moore HP, Machen TE. 2001. Mechanisms of pH regulation in the regulated secretory pathway. The Journal of biological chemistry 276: $33027-33035$.

Yamamoto K, Oota I. 1967. An electron microscopic study of the formation of the yolk globule in the oocyte of zebrafish, Brachydanio rerio. Bull Fac Fish, Hokkaido Univ 17: 165-174.

Yamashiro DJ, Tycko B, Fluss SR, Maxfield FR. 1984. Segregation of transferrin to a mildly acidic ( $\mathrm{pH}$ 6.5) para-Golgi compartment in the recycling pathway. Cell 37: 789-800.

Zeigler M, Meiner V, Newman JP, Steiner-Birmanns B, Bargal R, Sury V, Mengistu G, Kakhlon O, Leykin I, Argov Z et al. 2014. A novel SCARB2 mutation in progressive myoclonus epilepsy indicated by reduced beta-glucocerebrosidase activity. Journal of the neurological sciences 339: 210-213.

Zhao Y, Ren J, Padilla-Parra S, Fry EE, Stuart DI. 2014. Lysosome sorting of betaglucocerebrosidase by LIMP-2 is targeted by the mannose 6-phosphate receptor. Nature communications 5: 4321. 


\section{Curriculum Vitae}

Name: Palsamy Kanagaraj

Date of Birth: 15.11.1985

Place of Birth: Tamil Nadu, India

Address: Robert Koch Str. 38, app 829. 37075, Göttingen, Germany

Email: kanagaraj_426@yahoo.co.in

\section{Education and Research:}

10.2011 - 09.2014 Doctoral studies: "Souffle regulates secretory granule maturation by sorting lysosomal cargo from ISG during zebrafish oogenesis". Dept. of Developmental Biochemistry, University of Göttingen, Germany. Advisor: Dr. Roland Dosch.

06.2008 - 05.2011 Senior Research Associate: "Functional characterization of parasitism specific genes in plant parasitic nematode and interacting partners in plants and germ cell development in C.elegans". Biological sciences and bioengineering, Indian Institute of Technology (IIT), Kanpur, India.PI: Prof. Dr. K. Subramaniam.

07.2006 - 06.2008 Master studies: "Identification of molecular markers linked to drought resistance traits through bulk segregant analysis in rice". Center for Plant Molecular biology and Biotechnology, Tamil Nadu Agricultural University, Coimbatore. India.

Advisor: Prof. Dr. R. Chandra Babu, Dean, TNAU.

07.2002-04.2006 Bachelor studies: Agricultural college and research institute, Killikulam, TNAU, India.

\section{Awards and Stipends:}

1. GGNB Excellence Fellow (2012-Present)

2. DAAD fellowship for $\mathrm{PhD}$ program (2011-2014)

3. DBT Fellowship from Department of Biotechnology, Government of INDIA.

4. Young researcher award-2008 from IISc. Bangalore, India.

5. Indian Govt. Merit scholarship (2002-2006) 


\section{Publications:}

Sheetal babu paul, Biji. K. R, Senthil. A, Kanagaraj. P and Chandra babu. R. 2009. Identification of molecular marker for drought resistance using bulk line analysis. Int. Jour. Plant Arch., 9(1).

Kanagaraj. P, Biji. K. R, Silvas jebakumar prince. K, Annie sheeba. J, Senthil. A and Chandra babu. R. 2010.Identification of molecular marker linked to drought resistance in rice using Bulked segregant analysis. Curr. Sci. Vol. 98 No. 6, p836839.

Aravind kumar, Poornima, Silvas jebakumar, P. kanagaraj, Suji. K, Amudha.K, Senthil. A and Chandra babu.R. 2011. Fine Mapping QTL for Drought Resistance Traits in Rice (Oryza sativa L.) Using Bulk Segregant Analysis. Mol. Biotech, 49:90-95 .

K.K. Suji, K. Silvas Jebakumar Prince, P. Sumeet Mankhar, P. Kanagaraj, R. Poornima, K. Amutha, S. Kavitha, K.R. Biji, S. Michael Gomez, R. Chandra Babu. 2012. Evaluation of rice (Oryza sativa L.) near iso-genic lines with root QTLs for plant production and root traits in rainfed target populations of environment. Field Crops Research, 137: 89-96

Silvas jebakumar, P. Kanagaraj, Annie sheeba, Poornima, A.Senthil, S.Senthilvel and R.Chandra babu. 2014. Mapping QTLs for plant production traits under rainfed conditions predominant in target populations of environment using locally adapted indica rice (Oryza sativa L.) lines. Environmental and experimental botany (In Review).

Palsamy kanagaraj, Amandine Gautier- Stein, Dietmar Riedel, Christoph Schomburg, Joan cerda, franck Bontems, Nadine Vollock and Roland Dosch. 2014. Souffle/Spastizin controls secretory dense-core vesicle maturation during zebrafish oogenesis. PLoS Genet 10(6).

Palsamy Kanagaraj, Dietmar Riedel, and Roland Dosch. 2015. High pressure freezing and electron microscopy for analyzing zebrafish oogenesis. Methods in Molecular Biology. (Accepted)

Palsamy kanagaraj, Dietmar Riedel, Joan Cerda and Roland Dosch. 2015. Souffle/Spastizin regulates lysosomal cargo sorting from immature secretory granule. (In Preparation) 